\title{
New Methods for Studying Materials Under Shear with Nuclear Magnetic Resonance
}

by

Timothy Ian Brox

\author{
A thesis \\ submitted to the Victoria University of Wellington \\ in fulfilment of the \\ requirements for the degree of \\ Doctor of Philosophy \\ in Physics.
}

Victoria University of Wellington

2016 



\begin{abstract}
For over 30 years, nuclear magnetic resonance (NMR) techniques have been used to study materials under shear. Collectively referred to as Rheo-NMR, these methods measure material behaviour due to external stimuli and provide spatially and temporally resolved maps of NMR spectra, intrinsic NMR parameters (e.g. relaxation times) or motion (e.g. diffusion or flow). As a consequence, Rheo-NMR has been established as a complementary technique to conventional rheological measurements. In this thesis, new hardware and experimental methods are presented with the goal of advancing this exciting field through further integration of traditional rheometry techniques with NMR experiments.

Three key areas of hardware development have been addressed, including: 1) integrating torque sensing into the Rheo-NMR experiment for simultaneous bulk shear stress measurements, 2) constructing shear devices with geometric parameters closer to those used on commercial rheometers and 3) implementing an advanced drive system which allows for new shear profiles including oscillatory shear.

In addition to presenting the design and construction of various prototype instruments, results from validation and proof of concept studies are discussed. This information demonstrates that the hardware operates as expected and establishes an experimental parameter space for these new techniques.

Furthermore, these methods have been applied to open questions in various physical systems. This includes exploring the influence of shear geometry curvature on the onset of shear banding in a wormlike micelle surfactant system, observing shear induced structural changes in a lyotropic nonionic surfactant simultaneously via deuterium spectroscopy and bulk viscosity as well as studying interactions of flowing granular materials. The interpretation and implication of these observations are discussed in addition to motivating further studies.
\end{abstract}




\section{Acknowledgements}

It has been an absolute privilege to study in the Victoria University NMR lab. The quality and calibre of my colleagues has driven me to become a better scientist, but also, more importantly, a better person. This group has made me realise how much I love the challenges of research and for this gift I wish to recognise so many people.

For fostering an environment were folks are given the opportunity to pursue their passions I thank my supervisor, Petrik Galvosas. Petrik I will always appreciate your frank and honest approach to discussions. I value your feedback, but appreciate your willingness to allow me to make my own decisions. Over the years, you have strongly supported my academic pursuits, but also reminded me of the need for life-work balance.

To my fellow PhD students, at Vic and abroad, Fangrong Zong, Huabing Liu, Stefan Kuczera, Stefan Hertel, John Zhen, Wilfred Kittler, Hilary Fabich and Nathan Williamson, thank you all for keeping the bar high. Your contributions, suggestions and criticisms have been most welcome. Also, it has been a pleasure to work closely with visiting students, Dayle Jellyman, Robert Fischer, Finnian Gray, Leo Browning and Peter de Vrieze. Each one of you challenged me to become a more effective communicator and collaborator.

For providing me with the tools to perform quality research I am indebted to numerous researchers around the University and the Wellington region. Bradley Douglass, Sergei Obruchkov, Marcel Nogueira d'Eurydice and Achim Gädke, you are wizards of programming, electronics, physics and so much more. You have shown me not only how solve puzzles in the lab, but to take enjoyment in learning something new. Thank you all for your thoughtful suggestions and advice.

Around the world, but still a great mentor and wonderful friend, it has been amazing to continue working with my first NMR supervisor, Jennifer Brown. To you, Joe Seymour, Sarah Codd, Mark Skidmore and all the alumni of the MSU lab, I thank you for introducing me to the fantastic world of experimental science.

Among my New Zealand collaborators, I would like to thank Bill Williams, Mark Hunter and Daniel Holland for their enthusiasm, humour and keen insight. 
For helping me to get settled in the lab, in Wellington and in New Zealand, I must acknowledge the greatest kiwi ambassador, Phillip Luey. Please know how much I have appreciated your kindness and generosity (including your patience in introducing me to the sport of rugby).

Certainly I need to acknowledge (and apologise for) the terror and frustration I caused whenever I walked into the mechanical workshop with a stack of prints or turned up to the electronics shop (remarkably always five minutes before morning tea) with just "one quick question". Manu Pouajen-Blakiston, Nick Grinter, Alan Rennie and Rodney Brown, I am forever thankful for your patience, humour and continued support of my work.

To all of you, in no way do I believe our collaborations have ended and I look forward to many years as both friends and co-workers.

Pursuing my studies and academic passions in New Zealand has come at the great sacrifice of being far removed from my family. To all my family, please know that you are frequently in my thoughts and it was only with your love and support that I have achieved this milestone.

Finally, how can I even start to acknowledge my best friend, fellow scientist and wife, Bridget Brox? You know it's the rare moment that I am stumped for words, but I worry I might understate my feelings. Simply put, you mean the world to me and I look forward to moving into the next phase of our life, as always, hand in hand. 


\section{Contents}

1 Introduction 1

2 Rheology and Rheometry 5

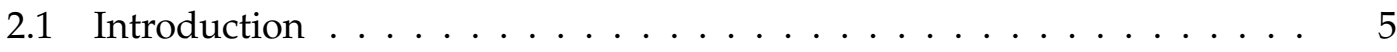

2.2 Tools for Modelling the Mechanical Behaviour of Materials . . . . . . . 6

2.2.1 Constitutive Equations . . . . . . . . . . . . . . . . . 6

2.2.2 Reference Frames . . . . . . . . . . . . . . . . . . 7

2.2 .3 Balance Equations . . . . . . . . . . . . . . . 8

2.2 .4 Boundary Conditions . . . . . . . . . . . . . 9

2.3 Deformation and Flow . . . . . . . . . . . . . . . . . 9

2.3 .1 Elastic Solid . . . . . . . . . . . . . . . . . . . . 9 9

2.3 .2 Viscous Liquid . . . . . . . . . . . . . . . . . . . . . 19

2.3 .3 Viscoelasticity . . . . . . . . . . . . . . . . 27

2.4 Measurements . . . . . . . . . . . . . . . . . . . . . 37

2.4.1 Rotational Drag Flow Rheometers . . . . . . . . . . . . . . . . 37

2.4.2 Rotational Drag Flow Geometries . . . . . . . . . . . . . . . 39

2.5 Conclusion . . . . . . . . . . . . . . . . . . . 47

3 Nuclear Magnetic Resonance $\quad 51$

3.1 Introduction . . . . . . . . . . . . . . . . . . 51

3.2 Nuclear Magnetism . . . . . . . . . . . . . . . . . . . . . 51

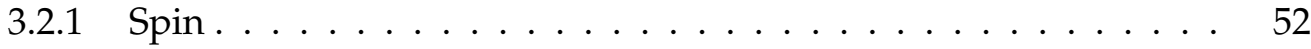

3.2 .2 Precession . . . . . . . . . . . . . . . . . 54

3.3 Fundamentals of an NMR Experiment . . . . . . . . . . . . 55

3.3.1 Excitation, Detection and Relaxation . . . . . . . . . . . . . . . . 57

3.3.2 Free Induction Decay . . . . . . . . . . . . . . . . . . . . . 60

3.3.3 Spin Echo . . . . . . . . . . . . . . . . . . . . . . 61

3.3.4 Signal Averaging . . . . . . . . . . . . . . . . . . . 62

3.3 .5 Phase Cycling . . . . . . . . . . . . . . . . . . 63

3.4 NMR Spectroscopy . . . . . . . . . . . . . . . . . . . . . 64 
3.4 .1 Proton Spectroscopy . . . . . . . . . . . . . . . . . . . . . . . . . . . . 64

3.4 .2 Deuterium Spectroscopy . . . . . . . . . . . . . . . . . 65

3.5 Relaxometry . . . . . . . . . . . . . . . . . . 65

3.5.1 Spin-Lattice Relaxation . . . . . . . . . . . . . . 65

3.5 .2 Spin-Spin Relaxation . . . . . . . . . . . . . . . . . . 66

3.6 Magnetic Field Gradients . . . . . . . . . . . . . . . . . . 67

3.6.1 Selective Excitation . . . . . . . . . . . . . . . . . . . . 67

3.6 .2 Magnetic Resonance Imaging . . . . . . . . . . . . . . . . . . . . . . . . . . . . . . 69

3.6.3 NMR Velocimetry . . . . . . . . . . . . . . . . . . . . . . 72

3.7 The NMR Instrument . . . . . . . . . . . . . . . . . . . . . . . . . . . . . . . . . . . . . . . . .

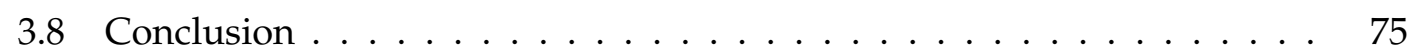

4 Previous NMR Studies of Materials Under Shear $\quad 77$

4.1 Introduction . . . . . . . . . . . . . . . . . . 77

4.2 Motion Encoding Pulse Sequences and NMR Signal . . . . . . . . . . . . . . . 78

4.3 Hardware (Drag Flow Shear) . . . . . . . . . . . . . . . . . . . . . 79

4.3.1 Commercially Available . . . . . . . . . . . . . . . . . . 79

4.3.2 Unique, Custom and One-Off . . . . . . . . . . . . . . . . 79

4.4 Emerging Techniques . . . . . . . . . . . . . . . . . . . . 81

4.4.1 Magnetic Resonance Elastography . . . . . . . . . . . . . . . . 81

4.4.2 Permanent Magnet NMR Rheology . . . . . . . . . . . . . 82

4.4 .3 Extensional Shear NMR . . . . . . . . . . . . . . . . . . . . . 83

4.4.4 Pulsed Second Order Field NMR . . . . . . . . . . . . . . . . . 84

4.5 Conclusion . . . . . . . . . . . . . . . . . . . 84

5 Instrument Design and Control Software $\quad 87$

5.1 Introduction . . . . . . . . . . . . . . . . . 87

5.1 .1 Improving Shear Geometries . . . . . . . . . . . . . . . 87

5.1 .2 New Experimental Protocols . . . . . . . . . . . . . . . . . . . . . . 88

5.1 .3 Simultaneous Bulk Rheometry . . . . . . . . . . . . . . . . 89

5.2 Shear Geometries . . . . . . . . . . . . . . . . . . . . . . . . . . . 89

5.2 .1 Concentric Cylinder . . . . . . . . . . . . . . . . . 89

5.2 .2 Parallel Disks . . . . . . . . . . . . . . . . . . . . . 95

5.2 .3 Planar-Cylindrical Hybrid . . . . . . . . . . . . . . . . . . . . . . . . . . . . . . . . . . . 97

5.3 Drive-Shaft Unit . . . . . . . . . . . . . . . . . . . . . . . . . . . . . 99

5.3.1 Overview (Drive-Shaft Generic) . . . . . . . . . . . . . . . . . 99

5.3.2 Super Wide Bore Variant . . . . . . . . . . . . . . . . . . . . . . 101

5.3 .3 Torque Sensor Variant . . . . . . . . . . . . . . . . . . . . 104

5.4 Electronics . . . . . . . . . . . . . . . . . . . . . . . . 107 
5.4.1 Eva Robotics ST-23 and EvoDrive . . . . . . . . . . . . . . . . 108

5.4.2 Arduino Due and Custom Shield . . . . . . . . . . . . . . . . . . 109

5.4.3 Torque Interface Card and Custom Electronics . . . . . . . . . . . 111

5.4 .4 Raspberry Pi Server . . . . . . . . . . . . . . . . . . 111

5.5 Software . . . . . . . . . . . . . . . . . . . 112

5.5 .1 Firmware . . . . . . . . . . . . . . . . . 113

5.5 .2 User Interface . . . . . . . . . . . . . . . . . . . . . . . . 114

5.6 Conclusion . . . . . . . . . . . . . . . . . . . 116

6 Hardware Validation Studies $\quad 117$

6.1 Introduction . . . . . . . . . . . . . . . . . . . 117

6.2 Shear Geometries . . . . . . . . . . . . . . . . . . . . . . 117

6.2.1 Concentric Cylinder (Super Wide Bore) . . . . . . . . . . . . . 117

6.2.2 Planar-Cylindrical Hybrid . . . . . . . . . . . . . . . . . . . . . . . 120

6.3 Drive-Shaft (Generic) . . . . . . . . . . . . . . . . . . . . . 122

6.3.1 Strain Rate Controlled Motion Profiles . . . . . . . . . . . . . . . . 122

6.3.2 NMR Acquisition Triggered by Motor . . . . . . . . . . . . . . . . 124

6.3.3 Torque Measurements (Vector Control) . . . . . . . . . . . . . . 127

6.4 Integrated Analogue Torque Sensor . . . . . . . . . . . . . . . . . . . . . 129

6.4.1 Data Sampling and Averaging . . . . . . . . . . . . . . . . . . 129

6.4 .2 Background Torque . . . . . . . . . . . . . . . . . . . . 131

6.4 .3 Stability and Drift . . . . . . . . . . . . . . . . . . . 132

6.4.4 Benchmark to Commercial Device . . . . . . . . . . . . . . . . 134

6.4.5 Simultaneous Torque and NMR Data Acquisition . . . . . . . . . 136

6.5 Conclusion . . . . . . . . . . . . . . . . . . . . . . . . . 139

$\begin{array}{lll}7 & \text { Applications } & 141\end{array}$

7.1 Overview . . . . . . . . . . . . . . . . . . . . . . . . . . . . . . 141

7.2 Influence of Couette Curvature on a Shear Banding Wormlike Micelle

Surfactant System . . . . . . . . . . . . . . . . . . . . . . . . . . . . . 142

7.2 .1 Introduction . . . . . . . . . . . . . . . . . . . . . . 142

7.2.2 Materials and Methods . . . . . . . . . . . . . . . . . . . . 144

7.2.3 Results and Discussion . . . . . . . . . . . . . . . . . . . 147

7.2 .4 Conclusions . . . . . . . . . . . . . . . . . . . . 151

7.3 Flow Observations of Granular Material . . . . . . . . . . . . . . . . . . 152

7.3.1 Motivation and Introduction . . . . . . . . . . . . . . 152

7.3.2 Experimental Methods . . . . . . . . . . . . . . . . . . . . 153

7.3.3 Preliminary Results and Discussion . . . . . . . . . . . . . 155 
7.4 Shear Induced Structural Transitions in a Lyotropic Nonionic Surfactant System . . . . . . . . . . . . . . . . . . . 157

7.4 .1 Introduction . . . . . . . . . . . . . . . . . 157

7.4 .2 Experimental Methods . . . . . . . . . . . . . . . . . . . 157

7.4 .3 Results and Discussion . . . . . . . . . . . . . . . . 158

8 Conclusion $\quad 161$

8.1 Work Presented in this Thesis . . . . . . . . . . . . . . . . . . . 161

8.2 Next Steps for Hardware Development . . . . . . . . . . . . . . . . . 162

8.2.1 High-Resolution Rheo-NMR . . . . . . . . . . . . . . . . . . 162

8.2.2 Stress Controlled Rheo-NMR . . . . . . . . . . . . . . . . . 163

8.2.3 Drive-Shaft Unit for Pressure Driven Shear and Extensional Rhe-

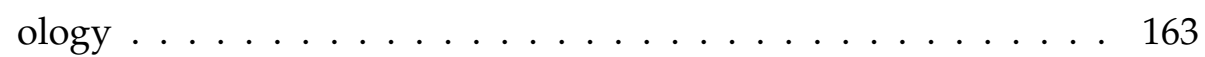

8.3 Final Remarks . . . . . . . . . . . . . . . . . . . . . . . . . . . . . 164

$\begin{array}{ll}\text { References } & 165\end{array}$

A Equations of Motion for a Viscous Fluid 181

A.1 Rectangular Coordinates . . . . . . . . . . . . . . . . . . . . . 181

A.2 Cylindrical Coordinates . . . . . . . . . . . . . . . . . . . . . . . . 181

A.3 Spherical Coordinates . . . . . . . . . . . . . . . . 182

B Mechanical Design $\quad 183$

B.1 Shear Geometries . . . . . . . . . . . . . . . . . . . . . . . . . . . . . . . . . . . . . . . . . . 183

B.2 Drive-Shaft Units . . . . . . . . . . . . . . . . . . . . . . 192

$\begin{array}{ll}\text { C PCB Design } & 197\end{array}$

C.1 Control Electronics . . . . . . . . . . . . . . . . . . . . . . . . 197

C.2 Torque Variant Electronics . . . . . . . . . . . . . . . . . . . 203

D motionControl Serial Commands $\quad 211$

D.1 Motion Commands . . . . . . . . . . . . . . . . . . . . . . . . 211

D.1.1 Stop . . . . . . . . . . . . . . . . . . 211

D.1.2 Single Value Shear Rate . . . . . . . . . . . . . . . . . . . . 212

D.1.3 Oscillatory Shear . . . . . . . . . . . . . . . . . . . . 213

D.1.4 Stepped Shear Rate . . . . . . . . . . . . . . . . . . . . . . . 213

D.1.5 Sawtooth . . . . . . . . . . . . . . . . . . . 214

D.1.6 Square Wave . . . . . . . . . . . . . . . . . . . . . . . 215

D.1.7 Stepped Motion . . . . . . . . . . . . . . . . . . . . . . . . 215

D.1.8 Single Value Shear Stress . . . . . . . . . . . . . . . . . . 216 
D.2 Parameters and Data Handling Commands . . . . . . . . . . . . . 216

D.2.1 Ping Motor . . . . . . . . . . . . . . . . . . 217

D.2.2 Motor Settings . . . . . . . . . . . . . . . . . . . . . . 218

D.2.3 SD Card/Data Options . . . . . . . . . . . . . . . . . . 222

D.2.4 Debug Mode . . . . . . . . . . . . . . . . . . . . . 223

D.2.5 Torque Sensor Calibration . . . . . . . . . . . . . . . . 227

D.2.6 Spectrometer Interface . . . . . . . . . . . . . . . . . . 228

D.2.7 Push Button Control . . . . . . . . . . . . . . . . . . . . . 230

D.3 Quick Reference . . . . . . . . . . . . . . . . . . . . . . . . . . . . 231 
CONTENTS 


\section{List of Figures}

2.1 The components on the total stress tensor acting on an arbitrary volume element. . . . . . . . . . . . . . . . . . . . . 11

2.2 Volume element with an arbitrary cut plane (illustrated by dashed line) where normal stress to the plane can be defined by the unit vector $\hat{\mathbf{n}}$ and the shear stress on the plane can be defined using the unit vector $\hat{\mathbf{s}}$. . . . 12

2.3 Volume element in the reference (a) and deformed states (b). . . . . . . . 13

2.4 A volume element seen in a reference configuration (a) is subjected to external forces $\mathbf{f}(\mathrm{b})$ which result in uniaxial extension (c). . . . . . . . 13

2.5 A volume element (a) is subjected to external forces $\mathbf{f}(\mathrm{b})$ which result in simple shear (c) . . . . . . . . . . . . . . . . . . .

2.6 The trajectories for two points embedded within a fluid (red dashed line). At some initial time the points could be found at $P$ and $Q$ with an infinitesimal separation defined by the vector $d \mathbf{X}$. At some later time these points could be found at $p$ and $q$ where they are now separated by $d \mathbf{x}$. At $p$ and $q$ each point (travelling with the fluid) has an instantaneous velocity (tangent to their respective trajectories) which differs from the other by $d \mathbf{v} \ldots \ldots \ldots \ldots \ldots \ldots \ldots \ldots \ldots \ldots \ldots \ldots \ldots \ldots \ldots$

2.7 Depiction of various fluid classes and their viscosity as a function of shear rate: constant (blue), shear-thickening (red) and shear-thinning (green). . . . . . . . . . . . . . . . . . .

2.8 Depiction of the shear stress response as a function of shear rate for various classes of fluids: constant viscosity (blue), Bingham plastic behaviour (black), shear-thickening (red) and shear-thinning (green). . . .

2.9 Material response to a hypothetical step stress experiment (actual experiments could not produce instantaneous change in applied stress). Single step stress input (top left); response of ideal elastic (Hookean) material (top right); ideal viscous (Newtonian) response (bottom left); viscoelastic material behaviour with initial jump in strain followed by increasing strain with time (bottom right). 
2.10 Number scale depicting the Deborah Number De (Equation 2.83) and corresponding material behaviours. . . . . . . . . . . .

2.11 Mechanical representation of the Kelvin-Voigt model of viscoelasticity where the spring element represents the pure elastic contribution and the dashpot the viscous component. . . . . . . . . . . . . .

2.12 Mechanical representation of Maxwell model for viscoelasticity. . . . . .

2.13 Ideal step strain experiment to illustrate the relaxation modulus of viscoelastic materials. Under a single step strain input (top left) the stress is shown as a function of time for an ideal elastic solid (top right), ideal viscous fluid (bottom left) and viscoelastic material (bottom right). . . .

2.14 Illustration of the Boltzmann Superposition Principle for viscoelastic materials. All plots show the strain history of a sample under test; single step strain $\Delta \gamma$ (top), two discrete strain steps (middle) and many steps can be treated as a sum of numerous steps (bottom) . . . . . . . . . . . .

2.15 Mechanical representation of Generalised Maxwell model for viscoelas-

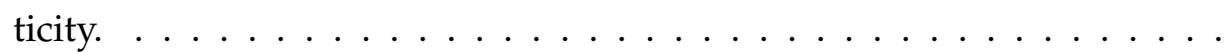

2.16 Illustration of an imposed oscillatory strain waveform and the stress response of a viscoelastic material. The top panel illustrates the sinusoidal input strain waveform which could alternatively be described by a sinusoidal shear rate waveform (bottom panel) which is phase shifted from the strain by $\frac{\pi}{2}$. The middle panel illustrates the stress response (solid line) which can be described as the sum of an elastic component (dashed line) and a solid component (dash dot line). . . . . . . . . . . . 36

2.17 Examples of commercial rheometers . . . . . . . . . . . . . . . 39

2.18 Simple schematic of a concentric cylinder (cylinderical Couette) shear geometry. . . . . . . . . . . . . . . . . 4 40

2.19 Measurement strategies for viscometer and rheometer instruments. . . . 41

2.20 Simple schematic of a cone and plate shear geometry. . . . . . . . . . . 46

2.21 Simple schematic of a parallel disks (plate and plate) shear geometry . .

3.1 Depiction of the energy levels for a spin-1/2 system due to the Zeeman interaction. . . . . . . . . . . . . . .

3.2 The expectation value for the spin vector $(\langle\boldsymbol{S}\rangle)$ in an external magnetic field $(\boldsymbol{B})$ precesses around the field at the Larmor frequency $(\omega)$ at a constant angle $(\alpha)$. Note this figure is not drawn to scale. . . . . . . . .

3.3 Depiction of individual spin vectors aligned parallel and anti-parallel with applied magnetic field B (a) and the net sum macroscopic magnetisation vector $\mathbf{M} \ldots \ldots \ldots \ldots \ldots \ldots \ldots$ 
3.4 Visualising the influence of the applied magnetic field $\mathbf{B}_{1}$ in the rotating frame. At thermal equilibrium the net magnetisation is aligned with the longitudinal axis and stationary (a); the magnetisation vector begins to precess around the applied $\mathbf{B}_{1}$ field (b); by varying the duration over which the $\mathbf{B}_{1}$ field is applied the tip angle of the magnetisation vector can be controlled, e.g. $90^{\circ}$ (c) or $180^{\circ}$ (d) . . . . . . . . . . . . . .

3.5 The precession of the net magnetisation $\mathbf{M}$ induces an $\mathcal{E} \mathcal{M F}$ in the resonant coil. . . . . . . . . . . . . . . . . . . . . . . 59

3.6 NMR pulse sequence for single pulse and signal acquisition. . . . . . . . 60

3.7 Real component (left) and imaginary component (right) of the NMR signal in a single pulse experiment. . . . . . . . . . . . . . . 61

3.8 Spin echo pulse sequence. . . . . . . . . . . . . . . . . . . . 62

3.9 Influence of the spin echo on spin evolution. . . . . . . . . . . . . 63

3.10 Visualising the real (blue) and imaginary (red) components of the FID signal in both the time domain (left) and frequency domain (right). . . . 64

3.11 Inversion recovery pulse sequence used to determine $T_{1} \ldots \ldots 65$

3.12 CPMG pulse sequence used to determine the $T_{2} \ldots \ldots \ldots$. . . . . . 67

3.13 Neglecting the $B_{o}$ field, the local magnetic field varies as a function of position along the $x$ axis due to an applied gradient field $\mathbf{G}(\boldsymbol{r})=\frac{\partial B_{z}}{\partial x} \hat{\mathbf{i}}$ (top). As a result of this gradient field, the local precession (neglecting the Larmor frequency due to $\mathbf{B}$ ) shows a similar spatially dependent pat-

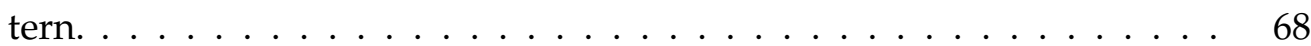

3.14 Example pulse programme for selective excitation. . . . . . . . . . .

3.15 Example magnetic resonance imaging data. Raw data for a 1D imaging experiment is shown in the top left plot, where the blue trace is the real part of the NMR signal and the red trace is the imaginary component. The reconstructed 1D projection of proton spin densities is shown in the top right plot. The panel in the bottom left shows the real component of the $2 \mathrm{D} k$-space data while the bottom right panel contains the real part of the real space image. . . . . . . . . . . . . . .

3.16 Pulse sequences for 1D imaging utilising either a frequency encoding scheme $(a)$ or a phase encoding scheme $(b) \ldots \ldots \ldots \ldots$

3.17 Two dimensional imaging pulse sequence which utilises both frequency and phase encoding. . . . . . . . . . . . . .

3.18 Basic pulse gradient spin echo (PGSE) pulse programme for encoding translation motion. . . . . . . . . . . . . . . . .

3.19 Bruker Wide Bore $400 \mathrm{MHz}$ superconducting magnet (spectrometer and operator console are not shown). . . . . . . . . . . . . . . 
4.1 Components of Magritek Rheo-NMR kit. . . . . . . . . . . . . . . . . 80

5.1 Various concentric cylinder shear devices. . . . . . . . . . . . . . . . . . . 91

5.2 Concentric cylinder device built for new drive-shaft unit. . . . . . . . . . 92

5.3 Parallel disks device built for new drive-shaft unit. . . . . . . . . . . . . . 96

5.4 Hybrid Couette shear cell (a) with regions of cylindrical and planar Couette flow. Stainless steel mold (b) for producing silicone bands (c) . . . . 98

5.5 Super wide bore drive-shaft unit with cross section. . . . . . . . . . . 100

5.6 Illustration of the super wide bore drive-shaft unit mounted inside a magnet shell. . . . . . . . . . . . . . . . . . 103

5.7 A Vibrac torque sensor was incorporated into a drive-shaft unit for bulk measurements during steady shear experiments. . . . . . . . . . . . . . . 104

5.8 The torque variant drive-shaft photographed during bench-top calibrations with cross section. The water bath was used to control the sample temperature. . . . . . . . . . . . . . . . . . 105

5.9 Illustration of the drive-shaft with inline torque transducer mounted in a wide bore magnet shell. . . . . . . . . . . . . . . . . 106

5.10 Block diagram of electronic components. . . . . . . . . . . . . . . 107

5.11 Arduino Due development board and custom shield mounted to the ST23 package for NMR experiments. . . . . . . . . . . . . . . . . 110

5.12 Vibrac torque interface board (back) shown connected to the Arduino Due and custom printed circuit boards (front). . . . . . . . . . . . 112

5.13 motionControl graphical user interface. . . . . . . . . . . . . . . . 115

5.14 TopSpin interface module. . . . . . . . . . . . . . . . . . 116

6.1 (a) Pulse program diagram for Pulsed Gradient Spin Echo (PGSE) motion encoding with double slice selection and 1D imaging acquisition. (b) Illustration of the imaging region. In this study, the $x$ axis aligns with gradient direction, the $y$ axis aligns with velocity direction and the $z$ axis aligns with vorticity direction. . . . . . . . . . . . . . . . 118

6.2 Normalised velocity measurements for three shear cells with the same stress variation across the gap (top); $\kappa=0.89, \frac{r_{\mathrm{i}}}{r_{\mathrm{o}}}=\frac{8.00}{9.00}$ (०), $\frac{11.10}{12.50}$ (॰), $\frac{15.10}{17.00}$ $(\bullet)$. And the two geometries with the greatest difference in stress variation across the gap (middle); $\kappa=0.71$ (॰) \& 0.96 (॰). The dashed line represents the theoretical velocity profile. $R^{2}$ values from comparing velocity profiles to the theoretical curve as a function of applied shear rate (bottom) . . . . . . . . . . . . . . . . . . . . . . . . . 119 
6.3 Comparision of 2D COMSOL simulation results (left column) to 2D NMR velocity images (right column). The top row compares the $x$ component of velocity while the bottom row compares the $y$ component of velocity.

6.4 Velocity profile for single gap (extracted from 2D NMR measurement at $x=0$ ) compared to straight line (theoretical model for simple shear). . .

6.5 Oscillatory shear waveform with angular position as a function of time (top); "Sawtooth" profile with motor frequency plotted as a function of time (middle); and example of stepped strain with motor frequency plotted as a function of time (bottom) $\ldots \ldots \ldots \ldots \ldots \ldots$

6.6 Pulse programme used for oscillatory $1 \mathrm{D}$ velocity imaging. . . . . . . . .

6.7 Pictorial representation of the NMR acquisition based on an oscillatory shear profile. . . . . . . . . . . . . . . . . . .

6.8 1D velocity profiles acquired using triggers based on the position of the motor shaft. To the right of the velocity map is an illustration of the applied sinusoidal waveform. . . . . . . . . . . . .

6.9 Uncorrected flow curves acquired using EvoDrive torque measurements and a 100k cSt sample at three fill heights (top); fill heights: 100 (॰), 71 (॰) and $45 \mathrm{~mm}(0)$. Flow curves ( 45 and $71 \mathrm{~mm}$ ) after data was normalised with the $100 \mathrm{~mm}$ "calibration" data (bottom). . . . . . . . . . . . . .

6.10 Viscosity calculated from flow curves acquired as a function of experiment time. The experiment time $t_{\exp }$ lists the length of data acqusition at each strain rate step. . . . . . . . . . . . . . . .

6.11 Torque data to show the magnitude of background torque as a function of rotation rate. . . . . . . . . . . . . . . . . . . .

6.12 Raw torque (top panel) and reference (middle panel) voltage signals; for both sets of data a running average has been calculated (black trace). Using the reference signal the torque data was corrected for drift. The bottom panel compares the raw torque signal (blue trace) to the drift compensated torque data (red trace) . . . . . . . . . . . . . . .

6.13 Rheology flow curves for three silicone oils, measured by NMR compatibile rheometer (markers) and compared to viscosity determined from commercial rheometer (dashed lines); nominal viscosities: 10k (०), 60k (॰) and 100k cSt (๑) . . . . . . . . . . . . . . . . . . . . . . . 
6.14 Simultaneous rheological flow curves and 1D NMR velocimetry for two samples; silicone oil (top row) and wormlike micelle solution (bottom row). The shading of the markers between flow curve (left) and velocity data (right) indicate the applied shear rate. In the case of the silicone oil, a straight line has been fit to the data to demonstrate the expected Newtonian behaviour. For the wormlike micelle sample, a second flow curve (○) was acquired with a commercial rheometer for comparison. . .

7.1 Concentric cylinder sampling matrix illustrating the various geometries used in the current study. The dashed line denotes shear cells with an identical stress variation across the gap. The two stars indicate the geometries with simultaneous torque measurements. . . . . . . . . . . .

7.2 Example data for WLM before and after the onset of shear banding. These velocity profiles come from the shear cell with $r_{\mathrm{i}}=8 \mathrm{~mm}$ and $r_{\mathrm{o}}=9 \mathrm{~mm}$. The lower shear rate $\left(2 \mathrm{~s}^{-1} ;\right.$ o) demonstrates homogeneous flow while shear banded flow can be seen in measurements at $20 \mathrm{~s}^{-1}$ (०).

7.3 Local shear rate of the "slowest" portion of the fluid domain, i.e. the half of the fluid gap farthest from the rotating wall, in shear cells with the same stress variation. All Couette geometries had a $\kappa=0.89 ; \frac{r_{\mathrm{i}}}{r_{\mathrm{o}}}=\frac{8.00}{9.00}$ $(\odot), \frac{11.10}{12.50}(\bullet), \frac{15.10}{17.00}(\bullet), \frac{21.00}{23.65}(\circ) \ldots \ldots \ldots \ldots \ldots \ldots$

7.4 Local shear rate of the "slowest" portion of the fluid domain, i.e. the half of the fluid gap farthest from the rotating wall, in the Couette geometries with $\kappa=0.71$ (०) \& $0.96(0) . \ldots \ldots \ldots$. . . . . . . . . . .

7.5 Local shear rate of the "slowest" portion of the fluid domain, i.e. the half of the fluid gap farthest from the rotating wall, in the Couette geometries with $\kappa=0.67$ (o) \& $0.89\left(r_{\mathrm{i}}=8.0 \mathrm{~mm} ; r_{\mathrm{o}}=9.0 \mathrm{~mm} ; 0\right)$. For these geometries, the NMR experiments were coupled with simultaneous torque measurements. . . . . . . . . . . . . . . . . . . . .

7.6 Flow curve data for commercial rheometer (o) and torque data collected simultaneously with $1 \mathrm{D}$ NMR velocimetry measurements, $\kappa=0.67$ (॰) \& $0.89\left(r_{\mathrm{i}}=8.0 \mathrm{~mm} ; r_{\mathrm{o}}=9.0 \mathrm{~mm} ; 0\right) \ldots \ldots \ldots \ldots \ldots$

7.7 (a) Vitamin E capsules loaded in a concentric cylinder shear device (top cap not installed). The layer of seeds affixed to the glass tubing is visible at the top of the fluid domain. (b) Shear device loaded with lobelia seeds and mounted to super wide bore drive-shaft unit; electrical tape was used to prevent slip between the cap of the concentric cylinder and the glass tubing. . . . . . . . . . . . . . . . . . . 154

7.8 Pulse programme used for granular flow experiments. . . . . . . . . . 155 
7.9 (top row) Axial MRI data for the four granular material systems; (left to right) lobelia seeds, petunia seeds, vitamin E capsules and mustard seeds. All images were $60 \mathrm{~mm}$ by $60 \mathrm{~mm}$ ( 256 points by 256 points). (bottom row) Velocity profiles for the respective granular system. In each experiment the motor was rotated such that the tangential wall speed was $17.1 \mathrm{~mm} \mathrm{~s}^{-1}$; furthermore all velocity data were acquired with an encoding time $\Delta=5 \mathrm{~ms}$. The vertical dashed lines indicate the boundaries of the shear cell while the horizontal dashed line indicates the velocity of the moving wall. . . . . . . . . . . . . . . . . . . . 156

$7.10{ }^{2} \mathrm{H}$ spectra collected as a function of accumulated strain. . . . . . . . . 158

7.11 Transient viscosity as a function of accumulated strain. . . . . . . . . . . 159

B.1 Exploded view of concentric cylinder geometry with bayonet style connection. . . . . . . . . . . . . . . . . . . . . . . . . 184

B.2 Exploded view of concentric cylinder geometry which mounts directly to the drive-shaft unit to eliminate backlash. . . . . . . . . . . . . . . 185

B.3 Exploded view of concentric cylinder $(\mathrm{OD}<5 \mathrm{~mm})$ for high resolution NMR systems. . . . . . . . . . . . . . . . . . . . . . . . . . . . 186

B.4 Exploded view of TA Instrument AR 2000 concentric cylinder accessory. The three part mount piece fits within the peltier jacket accessory available from TA Instruments. . . . . . . . . . . . . . . . . . 187

B.5 Exploded view of concentric cylinder geometry for drive-shaft unit with integrated torque transducer. . . . . . . . . . . . . . . . . . 188

B.6 Exploded view of parallel disks geometry for new drive-shaft unit. . . . 189

B.7 Exploded view of parallel disks accessory which allows the shear geometries from the NMR shear device (Figure B.6) to be used on a TA Instruments AR 2000 rheometer. . . . . . . . . . . . . . . . . . . . 190

B.8 Exploded view of hybrid shear device. . . . . . . . . . . . . . . . . . . 191

B.9 Exploded view of generic (wide bore) drive-shaft unit. . . . . . . . . . 193

B.10 Exploded view of wide bore drive-shaft unit with integrated torque transducer. . . . . . . . . . . . . . . . . . . . . . . . . 194

B.11 Exploded view of super wide bore drive-shaft unit. . . . . . . . . . . . . 195

B.12 Exploded view of super wide bore upper shim barrel adaptor for mounting drive-shaft unit within magnet. . . . . . . . . . . . . . . . . . . . 196

C.1 rheoMotor v1 schematic . . . . . . . . . . . . . . . . . . . . . . . . . . 198

C.2 rheoMotor v1 PCB component layout . . . . . . . . . . . . . . . . . . 199

C.3 rheoMotor v1 bill of material . . . . . . . . . . . . . . . . . . . . 199

C.4 rheoDueShield v4 schematic . . . . . . . . . . . . . . . . . . 200 
C.5 rheoDueShield v4 PCB component layout . . . . . . . . . . . . . . . . . 201

C.6 rheoDueShield v4 bill of material . . . . . . . . . . . . . . . . . . . 202

C.7 rheoDueShield v2 (torque variant) schematic . . . . . . . . . . . . . . 204

C.8 rheoDueShield v2 (torque variant) PCB component layout . . . . . . . . 205

C.9 rheoDueShield v2 (torque variant) bill of material . . . . . . . . . . . 206

C.10 rheoInterface v2 (torque variant) schematic . . . . . . . . . . . . . . 207

C.11 rheoInterface v2 (torque variant) PCB component layout . . . . . . . . . 208

C.12 rheoInterface v2 (torque variant) bill of material . . . . . . . . . . . . 208

C.13 rheoPower v2 (torque variant) schematic . . . . . . . . . . . . . . . 209

C.14 rheoPower v2 (torque variant) PCB component layout . . . . . . . . . . . 210

C.15 rheoPower v2 (torque variant) bill of material . . . . . . . . . . . . . . . 210

D.1 Oscilloscope screen capture of SPI test message on MOSI line. . . . . . . 226 


\section{List of Tables}

2.1 A summary of how various disciplines contribute to the study of soft materials and their use in practical applications (adopted from course notes of the 14th European School on Rheology). . . . . . . . . . . . . . . . 6

2.2 The continuity equation for an incompressible fluid in three common co-

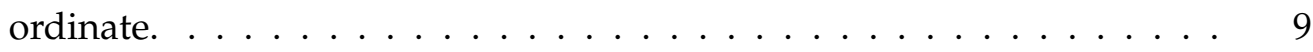

2.3 Review of Non-Newtonian fluid models. . . . . . . . . . . . . . . 25

2.4 Comparison of rotary drag flow shear geometries. . . . . . . . . . . . . 48

3.1 Larmor frequency as a function of magnetic field strength for proton and deuterium NMR . . . . . . . . . . . . . . . . . 55

5.1 Concentric cylinder shear cell combinations available with constructed components. Listed in the table is the gap and in parenthesis the $\kappa$ value of a Newtonian fluid for each rotor and cup pair. . . . . . . . . . . . .

6.1 Viscosity data for three silicone oils measured by a commercial instrument and the drive-shaft unit with inline torque transducer. . . . . . . . .

7.1 Mean diameter for particles and the dimensions of the concentric cylinder geometries used for studies of granular flow. . . . . . . . . . . . . 153 
$X X$ LIST OF TABLES 


\section{Chapter 1}

\section{Introduction}

At some point in life everyone is introduced to the idea that "stuff" comes in a variety of flavours (e.g. gas, liquid, solid). However, it would be immensely difficult to group typical materials into only one of these three classes. Where does melted cheese belong? How about shaving cream? Over the course of the day the average person interacts with numerous materials in a wide range of scenarios; from brushing one's teeth with toothpaste to drafting emails with the use of a LCD (liquid crystal display) screen, many everyday items have complex responses to external stresses. Further examples include food products, cosmetics, materials used in petrochemical and mineral resource recover, and even biological materials such as blood, sciatic fluid and DNA.

Understanding the dynamic response of materials, including polymers, liquid crystals, emulsions and micelles, has a wide range of applications in consumer products but is also a key focus of fundamental physics and the study of condensed matter. To mirror the diversity of materials and their characteristic response there are numerous analytical techniques that can be used to observe their intrinsic properties. One wide spread technique includes the manipulation of nuclear spins, broadly referred to as nuclear magnetic resonance (NMR). While diagnostic medical applications (i.e. magnetic resonance imaging, MRI) is probably the most familiar use of this technique, this application to biological materials (e.g. tissue) is only the start of its potential in materials science.

For nearly thirty years NMR experiments have been conducted on deformed (e.g. flowed, stretched) materials so that dynamic responses could be measured as a function of the testing circumstances. To further advance this field, this thesis focuses on the development of new experimental methods to study materials under shear with NMR techniques and includes early applications to three relevant physical systems. Chapters 2-4 introduce relevant background material; Chapters 5-7 contain original work and results; and in Chapter 8 the thesis is concluded. 


\section{Chapter 2: Rheology and Rheometry}

In this chapter core concepts of rheology (the study of flow and deformation) and rheometry (the tools for measuring rheological response) will be introduced. Regarding materials under flow, conservation laws and physical constrains will be used to build models and constituent equations for basic materials (elastic solids and viscous liquids) then these concepts will be further refined to introduce viscoelasticity and complex material response. The chapter will conclude with an introduction to measurement techniques used to characterise various materials. A key emphasis will be placed on drag flow shear geometries, which will be the focus of the experimental work presented in this thesis. Additionally, typical experiments and commercial instruments will be presented.

\section{Chapter 3: Nuclear Magnetic Resonance}

This chapter will serve as a primer for the NMR methods utilised in the experimental chapters. Core principles such as nuclear spin and precession will introduce the basis of the NMR signal while concepts such as relaxation and magnetic field gradients help explain simple NMR experiments. Finally, special attention will be given to specific NMR measurements that were used in this thesis, including magnetic resonance imaging, velocimetry and spectroscopy.

\section{Chapter 4: Previous NMR Studies of Materials Under Shear}

To tie together the introductory concepts of the previous two chapters, this chapter will serve as a brief review of previous work which used NMR techniques to study materials under shear. In some literature this class of methods is referred to as Rheo-NMR. This review will focus on developments of NMR pulse sequences, specialty hardware and systems under investigation.

\section{Chapter 5: Instrument Design and Control Software}

This chapter will focus on the design and construction of the various components of the new Rheo-NMR hardware presented in this thesis. This work can be divided into four key sections, specifically: 1) shear geometries, 2) drive-shaft devices, 3) electronics and 4) software.

\section{Chapter 6: Hardware Validation Studies}

Based upon the prototype hardware developed (and introduced in the previous chapter) this chapter will explore validation and proof of concept measurements for the var- 
ious devices. As appropriate these measurements will be bench-marked to commercial instruments or other measurement techniques.

\section{Chapter 7: Applications}

This final experimental chapter includes examples of how the devices designed (Chapter 5) and validated (Chapter 6 ) in this thesis have been utilised to study various materials. Key systems of interest will include a shear-banding wormlike micelle, granular materials under shear flow and a mutlilamellar vesicle surfactant system.

\section{Chapter 8: Conclusion}

The concluding chapter will summarise the work presented in this thesis along with providing an outlook on future Rheo-NMR studies and final closing remarks. 


\section{Chapter 2}

\section{Rheology and Rheometry}

\subsection{Introduction}

Derived from the Greek root "rheos", meaning to flow, the term rheology was first used in 1920 by E. C. Bingham of Lafayette College in Easton, PA, USA [1]:

"Rheology is the study of the deformation and flow of material."

When the Society of Rheology was founded in 1929, the definition of rheology was officially recognised. To distinguish rheology from other subjects it was concluded that its definition would exclude the flow of electrons and heat. Additionally, as rheologists of the time were interested in the properties and constitution of the matter under investigation, hydrodynamics, aerodynamics and the classical theory of elasticity would similarly be excluded. Despite their official exclusion from the definition of rheology, the roots of the field certainly trace back to early work in fluid and solid mechanics with significant contributions pre-dating the field by more than 250 years (including work by Hooke and Newton). This chapter will illustrate how modern rheology has built upon the work of these pioneers to form fundamental models which describe the response for various classes of materials to external forces. In the second half of this chapter rheometry, the various instruments and physical measurements used to investigate these materials and their properties, will be introduced and discussed.

Practitioners in the field of rheology (rheologists) utilise simple forms of deformation and flow to study primarily complex materials. Experimental data can then be fitted to constitutive equations which describe the stress response of a material due to a strain or rate of strain. Having established a fundamental description of a material's response to external forces material scientists can apply this behaviour to complex flows (developed for simple materials in fluid mechanics). The interplay between these disciplines is summarised in Table 2.1. Of course this is not to suggest that these fields 


\begin{tabular}{ccc} 
Discipline & Material & Deformation \\
\hline Rheology & Complex fluids & Simple flows \\
Fluid mechanics & Simple fluids & Complex flows \\
Materials science & Complex fluids & Complex flows
\end{tabular}

Table 2.1: A summary of how various disciplines contribute to the study of soft materials and their use in practical applications (adopted from course notes of the 14th European School on Rheology, Leuven, Belgium; September, 2013).

work in isolation, in fact it is quite important for the fluid mechanician to understand rheological properties and for rheologists to understand flow mechanics. As such, in addition to exploring material behaviours this chapter will examine the flow fields (albeit relatively simple flows) associated with rheological measurements.

The formatting of this chapter roughly follows the introductory textbook Rheology: Principles, Measurements and Applications by C.W. Macosko [1] and draws from the curriculum of the 14th European School on Rheology (Leuven, Belgium; September, 2013). The notation used in this chapter follows the official conventions set by the Society of Rheology [2]. Finally, the information presented in this chapter is only a survey of the world of rheology and for a more thorough treatment, the reader is directed to introductory textbooks on rheology and rheometry $[1,3-7]$, continuum mechanics [8] and fluid mechanics [9].

\subsection{Tools for Modelling the Mechanical Behaviour of Materials}

Key tools for describing material behaviour will be introduced in this section. This includes constitutive equations, conservation laws, assumptions of boundary conditions and establishing appropriate frames of reference.

\subsubsection{Constitutive Equations}

In rheology constitutive equations describe the response of a material to external fields or forces. Examples of these relationships from other disciplines include electrical resistance or heat capacity. One of the ultimate goals of exploring the rheology of a material is to describe how the material will respond to applied stimuli; these models can be used to either describe how a material will behave in a practical situation (e.g. pumping a fluid at a chemical engineering plant) or to explain underlying physical properties (e.g. molecular dynamics).

In this chapter constitutive relationships will first be developed for true elastic solids and pure viscous liquids. These concepts will be the foundation underlying general 


\subsection{TOOLS FOR MODELLING THE MECHANICAL BEHAVIOUR OF MATERIALS7}

models for everyday materials.

Constitutive equations can be written in terms of either a force or energy balance. To understand the experimental methods developed and used within this thesis, constitutive relationships derived from a force balance are sufficient and this chapter will focus on these models in particular. However, the reader should be aware that in certain circumstances an energy balance may be more appropriate; one example is accounting for the energy lost by viscous heating in a flowing fluid. Additionally, energy balance equations can be derived from molecular arguments and provide a direct connection between macroscopic response and molecular theory.

\subsubsection{Reference Frames}

In this chapter, two reference frames will be used. The first, the material coordinate system or Lagrangian perspective, follows a material as it flows or deforms and is described by points embedded within the material. The second, the spatial coordinate system or Eulerian perspective, is described by points fixed in space. In general, material coordinates can be helpful when studying solids by using the displacement of a test particle (real or imaginary) within the material to describe the applied deformation. However, for fluids it is typical to describe how the material flows through a fixed point in space which requires spatial coordinates.

To identify the material coordinates, upper case letters will be used, with the position vector $\mathbf{X}$ and points $X_{i}$. Lower case letters will be used for the spatial coordinates, with $\mathbf{x}$ and $x_{i}$ representing a position vector and point, respectively.

Vector functions ${ }^{1}$ can be used to change between reference frames. The position function to transform the position vector from the material to spatial coordinates is described by

$$
\mathbf{x}=\mathbf{X}(\mathbf{X}, t)
$$

where the opposite transformation (spatial to material coordinates) is describe by

$$
\mathbf{X}=\mathbf{x}(\mathbf{x}, t)
$$

In continuum mechanics, a reference state is assumed such that at $t=0$ an arbitrary material point, described as $\mathbf{A}$, can be described in the spatial frame such that

$$
\mathbf{x}=\mathbf{X}(\mathbf{A}, 0)=\mathbf{A} .
$$

\footnotetext{
${ }^{1}$ As the same symbols are used to describe the vector and vector function it would be easy to confuse the quantity with the function and vice versa. Additionally, different sources use various symbols for the vector function. To help avoid confusion, whenever a vector function is used in this thesis this will be explicitly stated.
} 
Furthermore, in the material frame, displacement and velocity can be described by their respective functions $\mathbf{U}(\mathbf{X}, t)$ and $\mathbf{V}(\mathbf{X}, t)$; in the spatial frame, the corresponding functions are $\mathbf{u}(\mathbf{x}, t)$ and $\mathbf{v}(\mathbf{x}, t)$.

\subsubsection{Balance Equations}

Deformed and flowing materials must obey both conservation of mass and momentum. Conservation of mass can be used to develop the continuity equation ${ }^{2}$

$$
\frac{\partial \rho}{\partial t}=-\nabla \cdot \rho \mathbf{v}
$$

where $\nabla$ is the nabla operator, $\rho$ is the material density and $\mathbf{v}$ the velocity vector. In the case of an incompressible material, the density will remain constant in time and the continuity equation becomes

$$
\nabla \cdot \mathbf{v}=0
$$

in which the divergence of the velocity field is equal to zero. The continuity equation for an incompressible fluid in three common coordinates (Cartesian, cylindrical and spherical) can be found in Table 2.2.

The assumption of incompressibility is important not only for simplifying mathematical descriptions of material behaviour but also in measuring the physical response of a material to external stimuli. For an incompressible material, rheological measurements made at different ambient pressures will still yield the same results as ambient pressure does not lead to deformation. If this assumption was not valid rheologists around the world would not be able to reproduce each others work.

From conservation of momentum, the Cauchy momentum equation can be derived ${ }^{3}$

$$
\frac{\partial}{\partial t}(\rho \mathbf{v})=-\nabla \cdot \rho \mathbf{v v}+\nabla \cdot \boldsymbol{\sigma}+\rho \mathbf{g}
$$

In the case of laminar or unidirectional flow (e.g. simple shear) the nonlinear inertial term $\nabla \cdot \rho \mathbf{v v}$ can be ignored and the momentum equation is simplified

$$
\frac{\partial}{\partial t}(\rho \mathbf{v})=\nabla \cdot \boldsymbol{\sigma}+\rho \mathbf{g}
$$

where $\sigma$ is a tensor which describes all stresses acting on a body and $\mathbf{g}$ is the acceleration due to gravity. The components of the momentum equation (Equation 2.7) in common coordinate systems can be found in Appendix A. Combined with the continuity equation these equations are typically referred to as the equations of motion. Later in this chapter Equation 2.7 will be used to derive the Navier-Stokes equation for an incompressible viscous fluid.

\footnotetext{
${ }^{2}$ Derivation available [1, sec. 1.7.1].

${ }^{3}$ Derivation available $[1$, sec. 1.7 .2$]$.
} 


\begin{tabular}{cc} 
System & Continuity Equation \\
\hline Cartesian $(x, y, z)$ & $\nabla \cdot \mathbf{v}=\frac{\partial v_{x}}{\partial x}+\frac{\partial v_{y}}{\partial y}+\frac{\partial v_{z}}{\partial z}=0$ \\
Cylindrical $(r, \theta, z)$ & $\nabla \cdot \mathbf{v}=\frac{1}{r} \frac{\partial}{\partial r}\left(r v_{r}\right)+\frac{1}{r} \frac{\partial v_{\theta}}{\partial \theta}+\frac{\partial v_{z}}{\partial z}=0$ \\
Spherical $(r, \theta, \phi)$ & $\nabla \cdot \mathbf{v}=\frac{1}{r^{2}} \frac{\partial}{\partial r}\left(r^{2} v_{r}\right)+\frac{1}{r \sin \theta} \frac{\partial}{\partial \theta}\left(v_{\theta} \sin \theta\right) \frac{1}{r \sin \theta} \frac{\partial v_{\phi}}{\partial \phi}=0$
\end{tabular}

Table 2.2: The continuity equation for an incompressible fluid in three common coordinate.

\subsubsection{Boundary Conditions}

Along with the equations of motion and descriptions of material behaviour (constitutive equations) boundary conditions are needed to solve physical problems. Furthermore, in developing rheological tests and experimental configurations, simplifying assumptions regarding the boundary conditions are typically made. Included are the no-slip and no-penetration conditions in which it is assumed that the material being tested moves with the surfaces of the apparatus (rheometer) and does not cross over this boundary. Furthermore, a continuity of both shear and normal forces must exist from the test instrument and the sample under examination. In general, additional boundary conditions may be necessary (e.g. liquid-liquid boundary within multi-phase flow) but these concepts will not be explored in this thesis.

\subsection{Deformation and Flow}

This section will develop fundamental relationships (constitutive relationships) for various types of materials and their response to external forces, starting with ideal materials (elastic solid and viscous liquid) and extending these concepts to more sophisticated examples (e.g. viscoelasticity).

\subsubsection{Elastic Solid}

From experimental work in the $15^{\text {th }}$ century, R. Hooke noted that the extension of a spring $\Delta L$ was directly dependent on the weight $F$ of a suspended mass, $F \propto \Delta L$. Today this relationship has been developed into Hooke's Law,

$$
F=-k x
$$

where the restoring force of a spring $F$ is equivalent to the displacement $x$ multiplied by a constant $k$. In terms of materials science, this form of Hooke's Law yields nothing regarding intrinsic properties of a material as the scaling constant $k$ can change due to the geometry of the spring and is not uniquely determined by the material of the spring. 
In the subsequent sections, applied stresses and the resulting deformations will be used to develop an equivalent to Hooke's Law in three dimensions where the proportionality constant will be determined by the properties of the material under test.

\section{Stresses}

To develop models that describe material deformation (either discrete or continuous) the forces acting on any part of a given material need to be identified. For this purpose the total stress tensor $\sigma$ is used ${ }^{4}$,

$$
\boldsymbol{\sigma}=\left[\begin{array}{lll}
\hat{\mathbf{x}}_{1} \hat{\mathbf{x}}_{1} \sigma_{11} & \hat{\mathbf{x}}_{1} \hat{\mathbf{x}}_{2} \sigma_{12} & \hat{\mathbf{x}}_{1} \hat{\mathbf{x}}_{3} \sigma_{13} \\
\hat{\mathbf{x}}_{2} \hat{\mathbf{x}}_{1} \sigma_{21} & \hat{\mathbf{x}}_{2} \hat{\mathbf{x}}_{2} \sigma_{22} & \hat{\mathbf{x}}_{2} \hat{\mathbf{x}}_{3} \sigma_{23} \\
\hat{\mathbf{x}}_{3} \hat{\mathbf{x}}_{1} \sigma_{31} & \hat{\mathbf{x}}_{3} \hat{\mathbf{x}}_{2} \sigma_{32} & \hat{\mathbf{x}}_{3} \hat{\mathbf{x}}_{3} \sigma_{33}
\end{array}\right]
$$

where a numbered coordinate system has been used (e.g. $\hat{\mathbf{x}}_{1}, \hat{\mathbf{x}}_{2}$ and $\hat{\mathbf{x}}_{3}$ in place of $\hat{\mathbf{x}}, \hat{\mathbf{y}}$ and $\hat{\mathbf{z}}$ for a Cartesian coordinate systems). For each component of the tensor, the first unit vector denotes the direction of the plane normal vector on which a stress acts and the second unit vector describes the direction of the stress. Tensors are mathematical devices used to transform a vector. Following this description the vector dot product of a plane normal vector $\hat{\mathbf{n}}$ with the total stress tensor $\boldsymbol{\sigma}$ will yield a stress vector $\mathbf{t}_{n}$ which describes the three components of stress acting on the plane

$$
\mathbf{t}_{n}=\hat{\mathbf{n}} \cdot \boldsymbol{\sigma}=\left[\begin{array}{lll}
n_{1} & n_{2} & n_{3}
\end{array}\right] \cdot\left[\begin{array}{lll}
\sigma_{11} & \sigma_{12} & \sigma_{13} \\
\sigma_{21} & \sigma_{22} & \sigma_{23} \\
\sigma_{31} & \sigma_{32} & \sigma_{33}
\end{array}\right]=\left[\begin{array}{l}
n_{1} \sigma_{11}+n_{2} \sigma_{21}+n_{3} \sigma_{31} \\
n_{1} \sigma_{12}+n_{2} \sigma_{22}+n_{3} \sigma_{32} \\
n_{1} \sigma_{13}+n_{2} \sigma_{23}+n_{3} \sigma_{33}
\end{array}\right]
$$

where, for convenience, the unit vectors (e.g. $\hat{\mathbf{x}}_{1}$ ) for the components of the normal vector $\hat{\mathbf{n}}$ and the stress vector $\mathbf{t}_{n}$ along with the unit double vectors (e.g. $\hat{\mathbf{x}}_{1} \hat{\mathbf{x}}_{1}$ ) for the total stress tensor are implied. The components of the stress tensor for an arbitrary volume are illustrated in Figure 2.1.

For the nine components of the total stress tensor illustrated in Figure 2.1, three will not produce a torque (i.e. the normal stresses $\sigma_{11}, \sigma_{22}$ and $\sigma_{33}$ ) while a torque can result from any of the remaining six components. However, due to conservation of angular momentum the moment created by any individual off diagonal element of the total stress tensor must be balanced. As a result, $\sigma_{i j}=\sigma_{j i}(i \neq j)$ and thus the total stress tensor is symmetric with six unique elements ${ }^{5}$. The notation in Equation 2.9 can then

\footnotetext{
${ }^{4}$ Within this chapter matrix notation will be used when representing tensors, but readers should be aware different conventions may be used in other sources [1, sec. 1.2.1].

${ }^{5} \mathrm{~A}$ non-symmetrical total stress tensor has been discussed in the literature, but never observed experimentally for amorphous liquids. In this derivation only body forces due to gravity have been considered; however, body torques can exist for particles in suspension [1, pp. 18 and ref. within] or due to electromagnetic forces (e.g. ferrofluids) but the treatment of these cases is unnecessary for this thesis.
} 


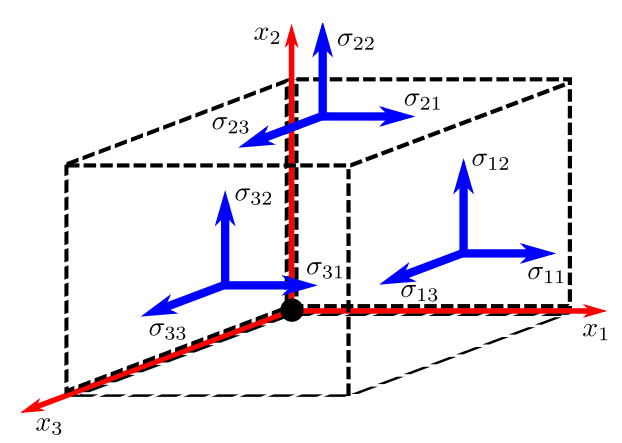

Figure 2.1: The components on the total stress tensor acting on an arbitrary volume element.

be reduced to

$$
\boldsymbol{\sigma}=\left[\begin{array}{lll}
\sigma_{11} & \sigma_{12} & \sigma_{13} \\
\sigma_{12} & \sigma_{22} & \sigma_{23} \\
\sigma_{13} & \sigma_{23} & \sigma_{33}
\end{array}\right] .
$$

Once the total stress tensor has been determined, a stress vector $\mathbf{t}_{n}$ can be calculated for any arbitrary plane (as illustrated in Figure 2.2) using Equation 2.10. Having determined the stress vector $\mathbf{t}_{n}$ acting on a plane the normal stress on the plane can be determined by a dot product (projection of $\mathbf{t}_{n}$ onto $\hat{\mathbf{n}}$ ),

$$
\sigma_{n n}=\mathbf{t}_{n} \cdot \hat{\mathbf{n}} .
$$

Finally, shear stress on the plane (orthogonal to the plane normal vector; defined by unit vector $\hat{\mathbf{s}}$ ) can be determined from vector subtraction,

$$
\sigma_{n s} \hat{\mathbf{s}}=\mathbf{t}_{n}-\sigma_{n n} \hat{\mathbf{n}} .
$$

In the case of a stationary fluid the only external forces will be the uniform normal stress from hydrostatic pressure $p$. In this case, the diagonal elements of total stress tensor $\left(\sigma_{11}, \sigma_{22}\right.$ and $\left.\sigma_{33}\right)$ are all equivalent and all other components are zero. Using an identity tensor $\mathbf{I}$, the total stress tensor can be defined

$$
\boldsymbol{\sigma}=-p \mathbf{I}
$$

where a negative sign is used to indicate compression from the hydrostatic pressure. Extending the concept above to a general case the total stress tensor can be defined

$$
\boldsymbol{\sigma}=-p \mathbf{I}+\boldsymbol{\tau}
$$

where $\tau$ is the extra stress tensor ${ }^{6}$. As discussed previously, via conservation of mass (Section 2.2.3), materials are typically assumed to be incompressible and thus external

\footnotetext{
${ }^{6}$ In some fluid mechanics literature $\tau$ is referred to as the viscous stress tensor.
} 


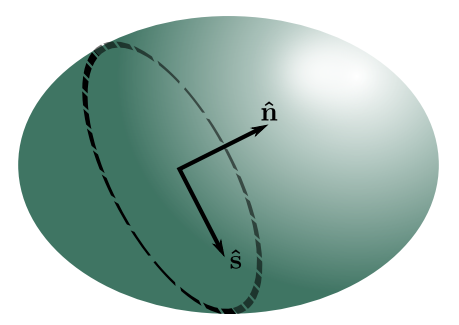

Figure 2.2: Volume element with an arbitrary cut plane (illustrated by dashed line) where normal stress to the plane can be defined by the unit vector $\hat{\mathbf{n}}$ and the shear stress on the plane can be defined using the unit vector $\hat{\mathbf{s}}$.

pressure does not lead to deformation. As a result, the extra stress tensor $\tau$ carries all information relating to material deformation. Despite most constitutive equations being developed using the extra stress tensor $\tau$, experimentally, it's only feasible to measure the total stress. As the pressure components of the total stress tensor are diagonal elements the shear stresses of the total stress tensor and extra stress tensor are equal, $\sigma_{i j}=\tau_{i j}(i \neq j)$, however, pressure will influence the normal stresses. As a solution, normal stress differences are reported

$$
\begin{aligned}
& \sigma_{11}-\sigma_{22}=\tau_{11}-\tau_{22} \\
& \sigma_{22}-\sigma_{33}=\tau_{22}-\tau_{33} .
\end{aligned}
$$

In the event of simple shear, which will be explored in the following section, the normal stress differences are referred to as $N_{1}$ and $N_{2}$ (Equations 2.16a and 2.16b, respectively).

\section{Discrete (Step) Deformations}

The total stress tensor developed in the previous section helps to describe stresses at any arbitrary point within a sample. This section will build similar tools to characterise discrete material deformations. For a material that has undergone deformation (Figure 2.3), a point in the reference configuration (defined with the materials coordinates $X_{1}$, $X_{2}$ and $X_{3}$ ) needs to be related to a position in the deformed state some time later (identified with the spatial coordinates $x_{1}, x_{2}$ and $x_{3}$ ).

The deformation tensor $\mathbf{F}$ characterises the change in spatial position with respect to the past position

$$
\begin{aligned}
& d \mathbf{x}=\frac{\partial \mathbf{x}}{\partial \mathbf{X}} \cdot d \mathbf{X} \\
& d \mathbf{x}=\mathbf{F} \cdot d \mathbf{X}
\end{aligned}
$$



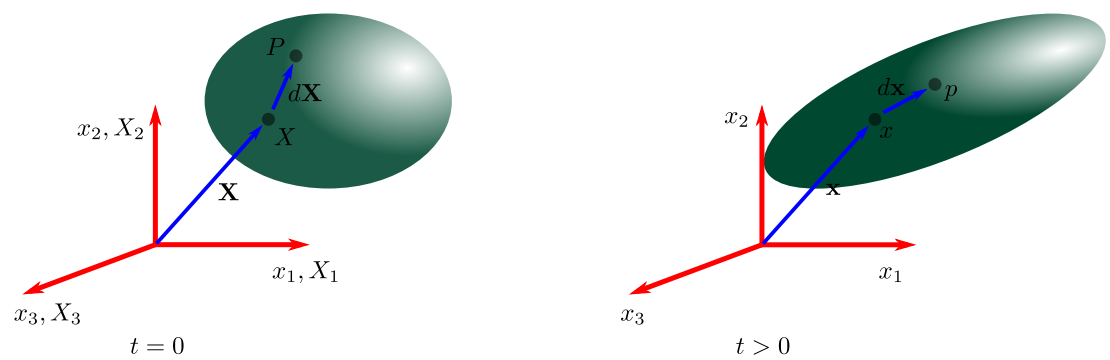

Figure 2.3: Volume element in the reference (a) and deformed states (b).

where the components of the deformation tensor (in Cartesian coordinates) are

$$
\mathbf{F}=\left[\begin{array}{lll}
\frac{\partial x_{1}}{\partial X_{1}} & \frac{\partial x_{1}}{\partial X_{2}} & \frac{\partial x_{1}}{\partial X_{3}} \\
\frac{\partial x_{2}}{\partial X_{1}} & \frac{\partial x_{2}}{\partial X_{2}} & \frac{\partial x_{2}}{\partial X_{3}} \\
\frac{\partial x_{3}}{\partial X_{1}} & \frac{\partial x_{3}}{\partial X_{2}} & \frac{\partial x_{3}}{\partial X_{3}}
\end{array}\right]
$$

Consider now the case of uniaxial extension (or compression), one of two simple deformations to be introduced in this chapter. An example of this deformation is the extension of a loaded bungee cord. In Figure 2.4a the reference, or initial, state of a rectangular volume element is depicted. Subjecting this body to tension forces along the $x_{1}$ direction $^{7}$ (Figure 2.4b) the resulting deformation can be seen in Figure 2.4c. As

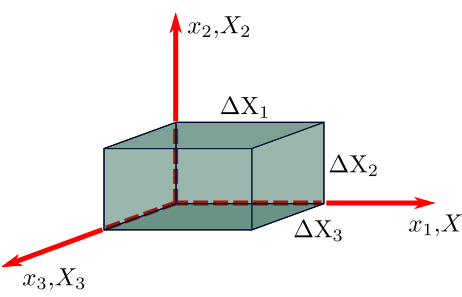

(a)

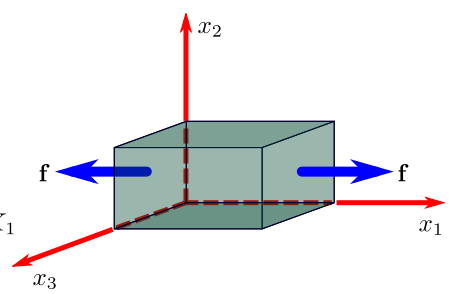

(b)

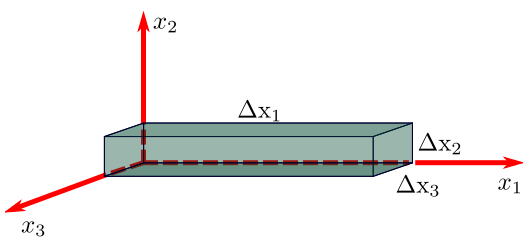

(c)

Figure 2.4: A volume element seen in a reference configuration (a) is subjected to external forces $\mathbf{f}(\mathrm{b})$ which result in uniaxial extension (c).

the deformation is homogeneous (i.e. deformation is the same at every point within material) the initial position of any point can be related to its position after deformation by an extension ratio for each axis

$$
x_{i}=\frac{\Delta \mathrm{x}_{i}}{\Delta \mathrm{X}_{i}} X_{i}=\alpha_{i} X_{i}
$$

Using the extension ratio along each axis $\alpha_{i}$, the deformation tensor for uniaxial exten-

\footnotetext{
${ }^{7}$ While tension on the $x_{1}$ normal planes are used to illustrate uniaxial extension, note that other combinations of external forces can result in this type of deformation.
} 
sion can be described by

$$
\mathbf{F}=\left[\begin{array}{ccc}
\alpha_{1} & 0 & 0 \\
0 & \alpha_{2} & 0 \\
0 & 0 & \alpha_{3}
\end{array}\right]
$$

For uniaxial extension, there is symmetry around the axis of extension $x_{1}$ such that the extension ratios $\alpha_{2}$ and $\alpha_{3}$ are equal. Furthermore, assuming the material is incompressible the volume remains unchanged

$$
\begin{aligned}
\Delta \mathrm{X}_{1} \Delta \mathrm{X}_{2} \Delta \mathrm{X}_{3} & =\Delta \mathrm{x}_{1} \Delta \mathrm{x}_{2} \Delta \mathrm{x}_{3} \\
1 & =\frac{\Delta \mathrm{x}_{1} \Delta \mathrm{x}_{2} \Delta \mathrm{x}_{3}}{\Delta \mathrm{X}_{1} \Delta \mathrm{X}_{2} \Delta \mathrm{X}_{3}} \\
1 & =\alpha_{1} \alpha_{2} \alpha_{3} \\
1 & =\alpha_{1} \alpha_{2}^{2} .
\end{aligned}
$$

Using the result from Equation 2.21, $\alpha_{2}$ can be defined in terms of $\alpha_{1}$

$$
\alpha_{2}=\sqrt{\frac{1}{\alpha_{1}}}=\alpha_{1}^{-1 / 2}
$$

and the deformation tensor can be rewritten in terms of $\alpha_{1}$

$$
\mathbf{F}=\left[\begin{array}{ccc}
\alpha_{1} & 0 & 0 \\
0 & \alpha_{1}^{-1 / 2} & 0 \\
0 & 0 & \alpha_{1}^{-1 / 2}
\end{array}\right]
$$

The second simple deformation to be considered is simple shear. To envisage simple shear consider a desk of cards stacked one on top of another. Where the top and bottom cards are displaced in opposite directions. This concept is illustrated in Figure 2.5. The first panel (Figure 2.5a) shows the initial state of a volume element and subsequently subjected to forces acting on the $x_{2}$ faces and directed along $x_{1}$ (Figure 2.5b) with the resulting deformation seen in Figure 2.5c. To relate the initial position of any point in

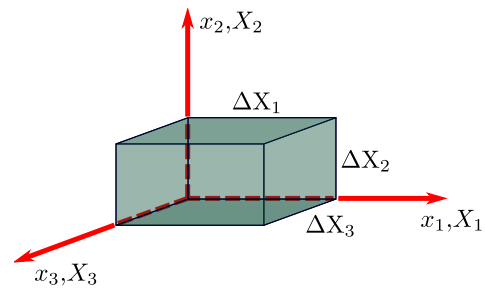

(a)

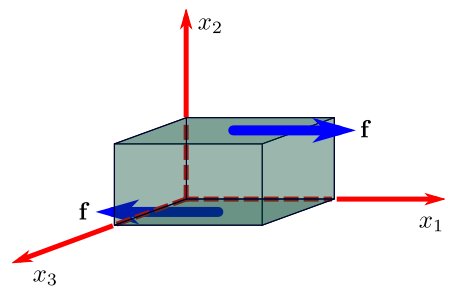

(b)

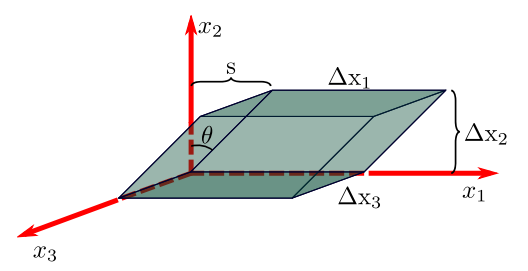

(c)

Figure 2.5: A volume element (a) is subjected to external forces $\mathbf{f}(\mathrm{b})$ which result in simple shear (c).

the volume to its position after the deformation, consider the descriptive example of 
the deck of cards with each card sliding over the card underneath it. Picture a point drawn on the face of any card; regardless of the initial position, its height within the stack doesn't change (i.e. $\Delta X_{2}=\Delta x_{2}$ ). Also, within the shear plane, the position of the point does not change along the axis perpendicular to the direction of shear. As the coordinate axes have been drawn in Figure 2.5 this suggests that there will be no change in the $x_{2}$ or $x_{3}$ directions. With regards to the $x_{1}$ dimension, the final position will be displaced from the initial position $X_{1}$ by a factor depending on the applied forces. As a result of the deformation, an angle $\theta$ can be defined between the $x_{2}$ axis and the side of the volume. For small displacements the angle can be estimated as

$$
\theta \approx \tan \theta=\frac{s}{\Delta \mathrm{X}_{2}}=\gamma
$$

where strain $\gamma$ is a dimensionless quantity to describe the applied external stresses. As a result, the position of any point within the volume experiencing simple shear would be described using

$$
\begin{aligned}
& x_{1}=X_{1}+\gamma X_{2} \\
& x_{2}=X_{2} \\
& x_{3}=X_{3}
\end{aligned}
$$

where the deformation tensor can be defined as

$$
\mathbf{F}=\left[\begin{array}{lll}
1 & \gamma & 0 \\
0 & 1 & 0 \\
0 & 0 & 1
\end{array}\right]
$$

Although not the case for the two examples worked above, in general, the deformation tensor $\mathbf{F}$ includes a description of both stretching and rotation ${ }^{8}$. As no deformation takes place during rigid body motion a more appropriate description of deformation is needed. Using the polar decomposition theorem of linear algebra ${ }^{9}$, the deformation tensor $\mathbf{F}$ can be treated as the product of a tensor describing deformation due to stretching (either $\mathbf{V}$ or $\mathbf{U}$ ) and a tensor describing rotation $\mathbf{R}$,

$$
\mathbf{F}=\mathbf{V} \cdot \mathbf{R}=\mathbf{R} \cdot \mathbf{U}
$$

where $\mathbf{V}$ and $\mathbf{U}$ are referred to as the left stretching tensor and right stretching tensor, respectively. Using the tensor property $(\mathbf{M} \cdot \mathbf{N})^{T}=\mathbf{N}^{T} \cdot \mathbf{M}^{T}$ the rotation tensor can be eliminated by the dot product of the deformation tensor with its transpose

$$
\begin{aligned}
& \mathbf{B}=\mathbf{F} \cdot \mathbf{F}^{T}=(\mathbf{V} \cdot \mathbf{R}) \cdot(\mathbf{V} \cdot \mathbf{R})^{T}=\mathbf{V} \cdot \mathbf{R} \cdot \mathbf{R}^{T} \cdot \mathbf{V}^{T}=\mathbf{V} \cdot \mathbf{I} \cdot \mathbf{V}^{T} \\
& \mathbf{C}=\mathbf{F}^{T} \cdot \mathbf{F}=(\mathbf{R} \cdot \mathbf{U})^{T} \cdot(\mathbf{R} \cdot \mathbf{U})=\mathbf{U}^{T} \cdot \mathbf{R}^{T} \cdot \mathbf{R} \cdot \mathbf{U}=\mathbf{U}^{T} \cdot \mathbf{I} \cdot \mathbf{U} .
\end{aligned}
$$

${ }^{8}$ This can be shown by creating a deformation tensor for a volume having experienced solid body rotation. In this case the deformation tensor would be equivalent to a rotation matrix.

${ }^{9}$ Worked example available [4, pp. 49]. 
The tensor product of a rotation tensor with its transpose will be unity (the transpose negates the initial rotation). Here two new tensors have been defined, the Finger tensor or the left Cauchy-Green tensor B and the Cauchy-Green tensor or the right Cauchy-Green tensor C (Equations 2.28a and 2.28b, respectively).

Physically, the Finger tensor gives information regarding the ratio between local area in the reference state $d \mathbf{A}$ to the deformed state $d \mathbf{a}$ through a series of operations on the normal vector of a plane in the deformed state $\hat{\mathbf{n}}$

$$
\frac{d \mathbf{A} \cdot d \mathbf{A}}{d \mathbf{a} \cdot d \mathbf{a}}=\hat{\mathbf{n}} \cdot \mathbf{B} \cdot \hat{\mathbf{n}} .
$$

Similarly, the Cauchy-Green tensor can be used to describe the change in lengths due to deformation. An extension ratio at any point in the material can be determined through operations on a normal vector in the reference configuration $\hat{\mathbf{N}}$

$$
\frac{d \mathbf{x} \cdot d \mathbf{x}}{d \mathbf{X} \cdot d \mathbf{X}}=\hat{\mathbf{N}} \cdot \mathbf{C} \cdot \hat{\mathbf{N}}
$$

Based on the two forms of deformation explored previously (uniaxial extension and simple shear) the Finger tensor and Cauchy-Green tensor can be calculated for each using Equations 2.28a and 2.28b. For uniaxial extension

$$
\mathbf{B}=\mathbf{C}=\left[\begin{array}{ccc}
\alpha_{1} & 0 & 0 \\
0 & \alpha_{1}^{-1 / 2} & 0 \\
0 & 0 & \alpha_{1}^{-1 / 2}
\end{array}\right] \cdot\left[\begin{array}{ccc}
\alpha_{1} & 0 & 0 \\
0 & \alpha_{1}^{-1 / 2} & 0 \\
0 & 0 & \alpha_{1}^{-1 / 2}
\end{array}\right]=\left[\begin{array}{ccc}
\alpha_{1}^{2} & 0 & 0 \\
0 & \alpha_{1}^{-1} & 0 \\
0 & 0 & \alpha_{1}^{-1}
\end{array}\right]
$$

and for simple shear

$$
\begin{gathered}
\mathbf{B}=\left[\begin{array}{lll}
1 & \gamma & 0 \\
0 & 1 & 0 \\
0 & 0 & 1
\end{array}\right] \cdot\left[\begin{array}{lll}
1 & 0 & 0 \\
\gamma & 1 & 0 \\
0 & 0 & 1
\end{array}\right]=\left[\begin{array}{ccc}
1+\gamma^{2} & \gamma & 0 \\
\gamma & 1 & 0 \\
0 & 0 & 1
\end{array}\right] \\
\mathbf{C}=\left[\begin{array}{lll}
1 & 0 & 0 \\
\gamma & 1 & 0 \\
0 & 0 & 1
\end{array}\right] \cdot\left[\begin{array}{lll}
1 & \gamma & 0 \\
0 & 1 & 0 \\
0 & 0 & 1
\end{array}\right]=\left[\begin{array}{ccc}
1 & \gamma & 0 \\
\gamma & 1+\gamma^{2} & 0 \\
0 & 0 & 1
\end{array}\right] .
\end{gathered}
$$

As illustrated in the examples above, both the Finger tensor and Cauchy-Green tensor are symmetrical.

Earlier in this section, Hooke's Law was introduced (Equation 2.8) in which a linear relationship was established between the restoring force and the applied extension of a spring. The scenario of a stretched spring (the basis for Hooke's experimental work) is not unlike the situation of uniaxial extension presented in Figure 2.4. In this case and using the coordinate notation established in previous examples, Hooke's Law could be rewritten as

$$
\sigma_{11}=E \epsilon
$$


where $\epsilon$ is the ratio of the change in length to the original length

$$
\epsilon=\frac{\Delta \mathrm{x}_{1}-\Delta \mathrm{X}_{1}}{\Delta \mathrm{x}_{1}}
$$

This ratio (engineering strain ${ }^{10}$ ) is scaled by a material property, Young's modulus $E$, to return the $\sigma_{11}$ component of the total stress tensor. This stress is the ratio of force divided by area for each face of the body on which the extension is applied. Practically Young's modulus can be determined for a material using the above model and either applying a defined force and measuring the resulting extension or vice versa. Despite the name uniaxial extension this model is valid for both extension and compression.

Alternatively, in the case of simple shear, Hooke's Law could be rewritten using the coordinate notation established in Figure 2.5

$$
\sigma_{12}=G \gamma
$$

where $\gamma$ (shear strain), previously defined in Equation 2.24 as the ratio between displacement and the height of the material $\frac{s}{\Delta X_{2}}$, is scaled by the material property, shear modulus $G$, to return the $\sigma_{12}$ component of the total stress tensor.

\section{Neo-Hookean Solid}

In the case of Hooke's Law for uniaxial extension (Equation 2.33), there is a linear relationship between stress and strain. This matches experimental data for ceramics and metals as these materials can typically only respond elastically for small strains (large strains can cause plastic deformation and fracture). However, for materials, like rubber, which can withstand large strains, a new model is needed. Utilising the total stress tensor and the Finger tensor the Neo-Hookean model can be defined as

$$
\boldsymbol{\sigma}=-p \mathbf{I}+G \mathbf{B}
$$

where the shear modulus $G$ is used to describe the intrinsic properties of the material under test. The origin of this model will be discussed in the following section.

Using the Finger tensor in the case of uniaxial extension (Equation 2.31) the NeoHookean model yields the following total stress tensor

$$
\boldsymbol{\sigma}=\left[\begin{array}{ccc}
-p+G \alpha_{1}^{2} & 0 & 0 \\
0 & -p+G \alpha_{1}^{-1} & 0 \\
0 & 0 & -p+G \alpha_{1}^{-1}
\end{array}\right]
$$

\footnotetext{
${ }^{10}$ Engineering strain is a convenience in which the original material dimensions, not the instantaneous dimensions, are used to describe strain; in the limit of very small deformations the engineering and true values of strain and stress are proportional.
} 
If no forces, other than hydrostatic pressure, are applied to the $x_{2}$ and $x_{3}$ faces, $\sigma_{22}$ and $\sigma_{33}$ equal zero and thus $p=G \alpha_{1}^{-1}$. Using this result, the $\sigma_{11}$ term can be defined

$$
\sigma_{11}=G\left(\alpha_{1}^{2}-\alpha_{1}^{-1}\right) .
$$

To compare the model above to the case of Hooke's Law applied to uniaxial extension (Equation 2.33) the extension ratio along the $x_{1}$ direction $\alpha_{1}$ needs to be converted to a strain. The extension ratio was previously defined in Equation 2.19 and combined with the definition of strain in Equation 2.34 yields $\alpha=\epsilon+1$. When substituted into the solution above yields

$$
\sigma_{11}=G\left(\frac{3 \epsilon+3 \epsilon^{2}+\epsilon^{3}}{1+\epsilon}\right)
$$

which is clearly a more complicated response than the linear behaviour suggested by Hooke's Law.

Now considering the case of $\epsilon \ll 1$, the Neo-Hookean model for uniaxial extension reduces to $\sigma_{11}=3 G \epsilon$, suggesting that in the limit of incompressible, isotropic ( $G$ equal in all directions) material under small strains

$$
E=3 G
$$

Here Young's modulus $E$ is three times the shear modulus $G$. Other relationships between these two parameters have been established, including the use of Poisson's ratio in the case of compressible materials, however, these will not be explored in this introductory chapter.

In the case of simple shear, the Neo-Hookean model combined with the Finger tensor found in Equation 2.32a yields a total stress tensor

$$
\boldsymbol{\sigma}=\left[\begin{array}{ccc}
-p+G\left(1+\gamma^{2}\right) & G \gamma & 0 \\
G \gamma & -p+G & 0 \\
0 & 0 & -p+G
\end{array}\right]
$$

The result of the $\sigma_{12}$ and $\sigma_{21}$ components match with the case of simple shear under Hooke's Law (Equations 2.35), however, the Neo-Hookean model makes an additional prediction. Previously it was discussed that normal stress differences are used to negate the influence of hydrostatic pressure on normal stresses and that for simple shear these stress differences are referred to as $N_{1}$ and $N_{2}$ (Equations 2.16a and 2.16b respectively)

$$
\begin{aligned}
& N_{1}=G \gamma^{2} \\
& N_{2}=0 .
\end{aligned}
$$

For an elastic material in simple shear, there will be a shear stress linear to the shear strain $\sigma_{12}=G \gamma$, a first normal stress difference which is a function of the square of the shear stain and a second normal stress difference equal to zero. A result that matches with experimental observations of materials such as rubber. 


\section{Hyperelastic Solid}

For some materials, the Neo-Hookean model is not sufficient to accurately describe the response of real materials. In particular these materials will deviate at large strains and may exhibit a non-linear stress to strain relationship. As a result, the framework for a general model of elastics was proposed by M. Mooney [10] and further explored by R.S. Rivlin [11]. Utilising the Finger tensor, the simple assumption is made that stress is a function of deformation

$$
\boldsymbol{\sigma}=f(\mathbf{B})
$$

Following the work of R.S. Rivlin [11], using a power series expansion, application of the Cayley-Hamilton theorem ${ }^{11}$ and simple algebra, the stress strain relationship can be defined as

$$
\boldsymbol{\sigma}=g_{0} \mathbf{I}+g_{1} \mathbf{B}+g_{2} \mathbf{B}^{-1}
$$

where $g_{0}, g_{1}$ and $g_{2}$ are functions of the invariants of the Finger tensor B. Discussion of tensor invariants will be held until discussion of a general model for viscous fluids later in this chapter. However, it is worthwhile to note that the Neo-Hookean model is one solution of the function above, where for an incompressible fluid, $g_{0}=-p, g_{1}=G$ and $g_{2}=0$. This function is the framework for numerous sophisticated models of elastic materials, however, these will not be explored in this thesis and the curious reader is instead referred to the textbook by R.W. Ogden [13].

\subsubsection{Viscous Liquid}

Having discussed elastic solids and models to explain their response to uniaxial extension and simple shear (i.e. Hooke's Law, Neo-Hookean and hyperelastic models) the focus will now be shifted to viscous liquids. Shortly after Hooke published his work on the extension of springs, Newton suggested that the local stress within a fluid is proportional to the change of velocity with respect to position (velocity gradient) scaled by a constant, known today as viscosity $\eta^{12}$. From Newton's work it took nearly 150 years to extend this constitutive relationship to three dimensions and this will be explored in the following section.

\footnotetext{
${ }^{11}$ Worked proof available [12, pp. 158].

${ }^{12}$ Alternatively, $\mu$ can be used to represent the dynamic viscosity, however, following the Official symbols and nomenclature of The Society of Rheology [2] this thesis will use $\eta$. Furthermore, do not let dynamic viscosity be confused with kinematic viscosity $\nu$ which is defined as ratio between dynamic viscosity and density $\nu=\frac{\eta}{\rho}$.
} 


\section{Continuous (Steady) Deformations}

In Figure 2.6 two arbitrary trajectories are represented (dashed lines) for two points embedded within the fluid (and thus moving along the streamlines within the fluid). At some past time, these points were found at $P$ and $Q$ where their separation could be described by the vector $d \mathbf{X}$. At a later time, these points are located at $p$ and $q$ with a separation $d \mathbf{x}$ and each had a respective velocity of $\mathbf{v}$ or $\mathbf{v}+d \mathbf{v}$. The quantity of interest is the difference in velocity between the two points and more generally, as described by Newton, how this separation in velocity $d \mathbf{v}$ varies with position. At any point within the fluid the velocity in spatial coordinates $\mathbf{v}$ is a function of position $\mathbf{x}$ and time $t$ and can be described by the vector function

$$
\mathbf{v}=\mathbf{v}(\mathbf{x}, t)
$$

Differentiating this velocity function with respect to position yields

$$
d \mathbf{v}=\frac{\partial \mathbf{v}}{\partial \mathbf{x}} \cdot d \mathbf{x}
$$

which describes the desired relationship, namely how the separation in position $d \mathbf{x}$ relates to the separation in velocity $d \mathbf{v}$. These terms are related by $\frac{\partial \mathbf{v}}{\partial \mathbf{x}}$, the velocity gradient tensor, which will be labelled $\mathbf{L}^{13}$

$$
d \mathbf{v}=\mathbf{L} \cdot d \mathbf{x}
$$

The velocity gradient tensor is defined (with the exclusion of the unit vectors) in Cartesian coordinates as

$$
\mathbf{L}=\left[\begin{array}{lll}
\frac{\partial v_{1}}{\partial x_{1}} & \frac{\partial v_{1}}{\partial x_{2}} & \frac{\partial v_{1}}{\partial x_{3}} \\
\frac{\partial v_{2}}{\partial x_{1}} & \frac{\partial v_{2}}{\partial x_{2}} & \frac{\partial v_{2}}{\partial x_{3}} \\
\frac{\partial v_{3}}{\partial x_{1}} & \frac{\partial v_{3}}{\partial x_{2}} & \frac{\partial v_{3}}{\partial x_{3}}
\end{array}\right]
$$

Previously the deformation tensor $F$ was used to characterise the change in present position with respect to a reference configuration. Taking the time derivative of the deformation tensor

$$
\dot{\mathbf{F}}=\frac{d}{d t}(\mathbf{F})=\frac{d}{d t}\left(\frac{\partial \mathbf{x}}{\partial \mathbf{X}}\right)
$$

where, by the symmetry of second derivatives, the order of derivatives can be switched

$$
\dot{\mathbf{F}}=\frac{\partial}{\partial \mathbf{X}}\left(\frac{d \mathbf{x}}{d t}\right)=\frac{\partial \mathbf{v}}{\partial \mathbf{X}}
$$

Applying the chain rule

$$
\dot{\mathbf{F}}=\left(\frac{\partial \mathbf{v}}{\partial \mathbf{x}}\right)\left(\frac{\partial \mathbf{x}}{\partial \mathbf{X}}\right)=\mathbf{L} \cdot \mathbf{F}
$$

\footnotetext{
${ }^{13}$ Alternatively, the velocity gradient tensor can be defined as the dyad product of the gradient operator $\nabla$ and the velocity vector $\mathbf{v}(\nabla \mathbf{v}$ or $\nabla \otimes \mathbf{v})$ which is equal to the transpose of $\mathbf{L}\left(\nabla \mathbf{v}=\mathbf{L}^{T}\right)$.
} 


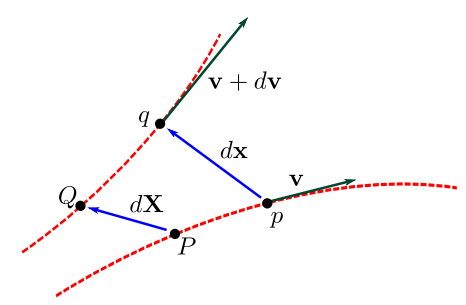

Figure 2.6: The trajectories for two points embedded within a fluid (red dashed line). At some initial time the points could be found at $P$ and $Q$ with an infinitesimal separation defined by the vector $d \mathbf{X}$. At some later time these points could be found at $p$ and $q$ where they are now separated by $d \mathbf{x}$. At $p$ and $q$ each point (travelling with the fluid) has an instantaneous velocity (tangent to their respective trajectories) which differs from the other by $d \mathbf{v}$.

where the time derivative of the deformation tensor is the product of the velocity gradient tensor and the deformation tensor. However, in the limit of instantaneous rate of separation $(t \rightarrow 0)$, the current state of the material $\mathbf{x}$ is the same as the reference configuration $\mathbf{X}$ such that

$$
\lim _{\mathbf{X} \rightarrow \mathbf{x}} \mathbf{F}=\mathbf{I}
$$

and thus,

$$
\lim _{\mathbf{X} \rightarrow \mathbf{x}} \mathbf{L}=\dot{\mathbf{F}} \text {. }
$$

From the relationship in Equation 2.53 it is evident that the velocity gradient tensor $\mathbf{L}$, like the deformation gradient $\mathbf{F}$, carries information regarding both stretching and rotation. Rigid body motion does not lead to deformation; in the previous sections the Finger Tensor and Cauchy-Green tensor were constructed such that any contribution from rotation was eliminated. Similarly, a rate of strain tensor $\dot{\gamma}$ can be constructed from the velocity gradient tensor $\mathbf{L}$. Using the product rule to take the time derivative of the relationship in Equation 2.27

$$
\dot{\mathbf{F}}=\dot{\mathbf{R}} \cdot \mathbf{U}+\mathbf{R} \cdot \dot{\mathbf{U}}
$$

where the right stretching tensor $\mathbf{U}$ has been used; the above relationship could have been rewritten in terms of the left stretching tensor $\mathbf{V}$ with the following result remaining unchanged. In Equation 2.52 it was shown that in the limit $\mathbf{X} \rightarrow \mathbf{x}$ there is no deformation $(\mathbf{F}=\mathbf{I})$. Applied to Equation 2.27, this suggests

$$
\lim _{\mathbf{X} \rightarrow \mathbf{x}} \mathbf{U}=\mathbf{R}=\mathbf{I} .
$$

which combined with Equation 2.53

$$
\mathbf{L}=\dot{\mathbf{R}}+\dot{\mathbf{U}}
$$


Thus the velocity gradient tensor is comprised of a rate of spin tensor $\dot{\mathbf{R}}$ and a symmetric rate of deformation tensor (alternatively the rate of stretching tensor) $\dot{\mathbf{U}}$. Previously it was discussed that $\mathbf{R} \cdot \mathbf{R}^{T}=\mathbf{I}$ suggesting that $\mathbf{R}^{T}=\mathbf{R}^{-1}$; thus adding the rate of spin tensor $\dot{\mathbf{R}}$ and its transpose $\dot{\mathbf{R}}^{T}$ will negate any spin

$$
\dot{\mathbf{R}}+\dot{\overline{\mathbf{R}^{T}}}=0 .
$$

As the rate of stretching tensor is symmetric,

$$
\dot{\mathbf{U}}=\dot{\mathbf{U}}^{T},
$$

combining Equations 2.56, 2.57 and 2.58 any contribution from rigid body motion can be eliminated using

$$
\dot{\gamma}=\mathbf{L}+\mathbf{L}^{T}
$$

Which defines the rate of strain tensor $\dot{\gamma}^{14}$.

With the appropriate tools at hand, Newton's equation for viscous fluids can be written as

$$
\boldsymbol{\sigma}=-p \mathbf{I}+\eta \dot{\gamma}
$$

From the examples in the previous sections the velocity gradient tensor $\mathbf{L}$ and rate of strain tensor $\dot{\gamma}$ can be calculated. In the case of steady uniaxial extension the velocity gradient tensor takes the form

$$
\mathbf{L}=\left[\begin{array}{ccc}
\dot{\alpha}_{1} & 0 & 0 \\
0 & \dot{\alpha}_{2} & 0 \\
0 & 0 & \dot{\alpha}_{3}
\end{array}\right]
$$

Again, due to the symmetry for uniaxial extension, about $x_{1}$ in these examples, $\dot{\alpha}_{2}=$ $\dot{\alpha}_{3}$. Also, assuming an incompressible fluid, the continuity equation $\nabla \cdot \mathbf{v}=0$ (the divergence of the velocity field is zero) can be used such that

$$
\dot{\alpha}_{1}+2 \dot{\alpha}_{2}=0 .
$$

Solving for $\dot{\alpha}_{2}$ in terms of $\dot{\alpha}_{1}$ yields

$$
\dot{\alpha}_{2}=-\frac{\dot{\alpha}_{1}}{2}
$$

such that

$$
\mathbf{L}=\left[\begin{array}{ccc}
\dot{\alpha}_{1} & 0 & 0 \\
0 & -\frac{\dot{\alpha}_{1}}{2} & 0 \\
0 & 0 & -\frac{\dot{\alpha}_{1}}{2}
\end{array}\right]
$$

\footnotetext{
${ }^{14}$ In some disciplines the rate of strain tensor is replaced with the rate of deformation tensor $\dot{\mathbf{U}}$ and recognised by the tensor $\mathbf{D}$ in which case note there is a factor of two difference $\dot{\gamma}=2 \mathbf{D}$. Additionally, some texts define $\dot{\gamma}$ with the product of the gradient operator and the velocity vector $\dot{\gamma}=\nabla \mathbf{v}+\nabla \mathbf{v}^{T}$
} 
Using the relationship in Equation 2.59, the rate of strain tensor becomes

$$
\dot{\gamma}=\left[\begin{array}{ccc}
2 \dot{\epsilon} & 0 & 0 \\
0 & -\dot{\epsilon} & 0 \\
0 & 0 & -\dot{\epsilon}
\end{array}\right] .
$$

Where the relationship between extension $\alpha$ and strain $\epsilon, \alpha=\epsilon+1$, has been used to replace $\dot{\alpha}$ with $\dot{\epsilon}$. Utilising Newton's constitutive relation (Equation 2.60)

$$
\boldsymbol{\sigma}=\left[\begin{array}{ccc}
-p+2 \eta \dot{\epsilon} & 0 & 0 \\
0 & -p-\eta \dot{\epsilon} & 0 \\
0 & 0 & -p-\eta \dot{\epsilon}
\end{array}\right]
$$

If surface tension effects can be neglected the boundary conditions on the free surface are such that

$$
\sigma_{22}=\sigma_{33}=0 .
$$

Combined with the total stress tensor for uniaxial extension (Equation 2.66) the only non-zero component is $\sigma_{11}$,

$$
\sigma_{11}=3 \eta \dot{\epsilon}
$$

If an extensional viscosity $\eta_{E}$ is defined

$$
\eta_{E}=\frac{\sigma_{11}-\sigma_{22}}{\dot{\epsilon}}
$$

for this example of uniaxial extension, the extensional viscosity is three times the shear viscosity,

$$
\eta_{E}=3 \eta
$$

a relationship known as Trouton's law.

In the instance of simple shear, the velocity gradient tensor can easily be solved

$$
\mathbf{L}=\left[\begin{array}{ccc}
0 & \dot{\gamma} & 0 \\
0 & 0 & 0 \\
0 & 0 & 0
\end{array}\right]
$$

with the rate of strain tensor

$$
\dot{\gamma}=\left[\begin{array}{ccc}
0 & \dot{\gamma} & 0 \\
\dot{\gamma} & 0 & 0 \\
0 & 0 & 0
\end{array}\right]
$$

and the resulting total stress tensor

$$
\boldsymbol{\sigma}=\left[\begin{array}{ccc}
-p & \eta \dot{\gamma} & 0 \\
\eta \dot{\gamma} & -p & 0 \\
0 & 0 & -p
\end{array}\right]
$$

In which the only contributions to normal stresses come from hydrostatic pressure. Additionally, there are shear stress components, $\sigma_{12}=\sigma_{21}=\eta \dot{\gamma}$. 


\section{General Viscous Fluid}

Analogous to the discussions of a general elastic model, a general viscous model can be developed from the simple assumption that stress is a function of rate of strain

$$
\boldsymbol{\sigma}=f(\dot{\gamma})
$$

Again, using a power expansion and the Cayley-Hamilton theorem, stress can be defined as a function of the rate of strain tensor $\dot{\gamma}$ and scalar functions $\eta_{i}{ }^{15}$

$$
\boldsymbol{\sigma}=\eta_{0} \mathbf{I}+\eta_{1} \dot{\gamma}+\eta_{2} \dot{\gamma}^{2}
$$

This relationship is commonly known as the Reiner-Rivlin constitutive equation and named for the contributions from M. Reiner [15] and R.S. Rivlin [16]. The terms $\eta_{1}, \eta_{2}$ and $\eta_{3}$ are functions themselves of the invariants ${ }^{16}$ of the rate of strain tensor

$$
\begin{aligned}
I_{\dot{\gamma}} & =\operatorname{tr}(\dot{\gamma}) \\
I I_{\dot{\gamma}} & =\frac{1}{2}\left[(\operatorname{tr} \dot{\gamma})^{2}-\operatorname{tr}\left(\dot{\gamma}^{2}\right)\right] \\
I I I_{\dot{\gamma}} & =\operatorname{det}(\dot{\gamma}) .
\end{aligned}
$$

\section{Generalised Newtonian Fluid Models}

The Riener-Rivlin constitutive equation (Equation 2.75) can be simplified through a few assumptions. Namely, for an incompressible fluid $\eta_{0}=-p$ and for simple flows (e.g. simple shear) $I_{\dot{\gamma}}=I I I_{\dot{\gamma}}=0$ such that $\eta_{1}$ becomes a function of only $I I_{\dot{\gamma}}$. Additionally, the third term in $\eta_{2} \dot{\gamma}^{2}$ predicts a first normal stress difference of zero which fails to match experimental data such that this second order term is typically discarded. Based on these simplifications, Equation 2.75 can be reduced to

$$
\boldsymbol{\sigma}=-p \mathbf{I}+\eta\left(I I_{\dot{\gamma}}\right) \dot{\gamma}
$$

Furthermore, having focused on steady simple shear the relationship above can be described without the tensor notation and can be simplified to

$$
\sigma_{12}=\eta\left(I I_{\dot{\gamma}}\right) \dot{\gamma}
$$

For the simplified equation above, in the case that $\eta$ is constant, this is the description of Newtonian behaviour (Equation 2.60). Alternatively, fluids in which the viscosity changes with the applied shear rate are referred to as Generalised Newtonian fluids. Various models are used to describe this Non-Newtonian behaviour with the most widely used discussed in Table 2.3.

\footnotetext{
${ }^{15} \mathrm{~A}$ through treatment of general viscous fluid models is available [14, pp. 256].

${ }^{16} \mathrm{~A}$ derivation of principal invariants is available [4, pp. 33]; tensor invariants are particularly useful for constitutive equations as their values remain constant regardless of what coordinate system is used to describe the tensor.
} 


\begin{tabular}{|c|c|c|}
\hline Name & Form & Comments \\
\hline Power law [14, chap. 17] & $\eta=k \dot{\gamma}^{n-1}$ & $\begin{array}{l}\text { Particularly useful for the process- } \\
\text { ing a range of polymeric liquids and } \\
\text { dispersions. The Power law is the } \\
\text { most common constitutive equation } \\
\text { for viscous fluids. There are two fit- } \\
\text { ting parameters } k \text { and } n \text { where } k \text { can } \\
\text { represent a function of temperature; } \\
n<1 \text { is referred to as shear-thinning } \\
\text { and } n>1 \text { shear-thickening (Figures } \\
2.7 \text { and } 2.8 \text { ). }\end{array}$ \\
\hline Sisko model [17] & $\eta=k_{1}+k_{2} \dot{\gamma}^{n-1}$ & $\begin{array}{l}\text { Originally developed for greases and } \\
\text { useful for other coating flows, the } \\
\text { Sisko model assumes there are flow } \\
\text { units; one Newtonian and one non- } \\
\text { Newtonian. There are three fitting } \\
\text { parameters } k_{1}, k_{2} \text { and } n \text { where } k_{1} \text { is } \\
\text { a constant to describe Newtonian be- } \\
\text { haviour at high shear rates while } k_{2} \\
\text { and } n \text { correspond to the fitting pa- } \\
\text { rameters of the Power law. }\end{array}$ \\
\hline Cross model [18] & $\frac{\eta-\eta_{\infty}}{\eta_{o}-\eta_{\infty}}=\frac{1}{1+(k \cdot \dot{\gamma})^{1-n}}$ & $\begin{array}{l}\text { The main feature of the Cross model } \\
\text { is asymptotic viscosities at very low } \\
\text { and very high shear rates, } \eta_{o} \text { and } \eta_{\infty}, \\
\text { respectively. Written in an alternative } \\
\text { form the Cross model is referred to } \\
\text { as the Carreau model [19]. For poly- } \\
\text { mers melts, } \eta_{\infty} \text { can be set to zero re- } \\
\text { ducing the total fitting parameters to } \\
\text { three, a form referred to as the Ellis } \\
\text { model [6, pp. } 18] \text {. }\end{array}$ \\
\hline
\end{tabular}

Table 2.3: Review of Non-Newtonian fluid models.

\section{Yield Stress Fluids}

In the case of the Sisko model (Table 2.3), if the fitting parameter $n$ is set to zero, the viscosity term $\eta$ in the simplified form of the Riener-Rivlin constitutive equation (Equation 


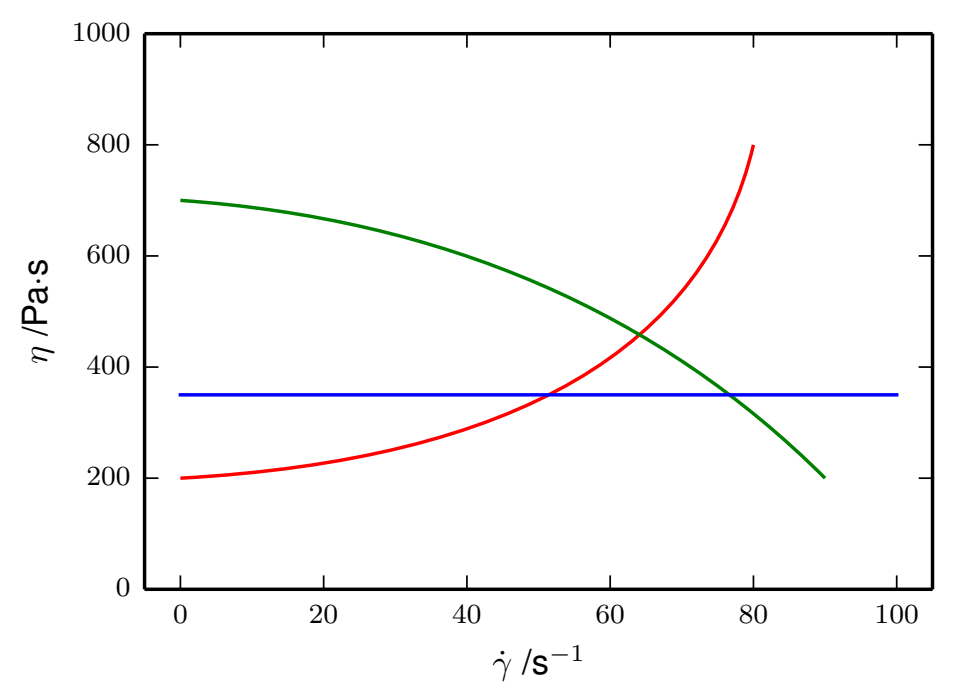

Figure 2.7: Depiction of various fluid classes and their viscosity as a function of shear rate: constant (blue), shear-thickening (red) and shear-thinning (green).

2.78) can be rewritten

$$
\eta=k_{1}+k_{2} \dot{\gamma}^{-1} \text {. }
$$

Which forms the constitutive equation for the Bingham model [20]

$$
\sigma_{12}=k_{1} \dot{\gamma}+k_{2}
$$

Where the term $k_{2}$ is referred to as the yield stress. In brief, the Bingham model suggests that a material acts as a rigid body below a critical shear stress; however above this value it acts as a viscous liquid. This behaviour can be described as a piecewise function

$$
\dot{\gamma}=\left\{\begin{array}{ll}
0 & \sigma_{12}<k_{2} \\
\frac{\sigma_{12}-k_{2}}{k_{1}} & \sigma_{12} \geq k_{2}
\end{array} .\right.
$$

The Bingham model is graphically demonstrated in Figure 2.8 in which the strain-stress relationship is linear above a critical shear stress. One alternative form of the Bingham model is the Herschel-Bulkley model $[1, \text { pp. } 97]^{17}$ in which the viscosity term $\eta\left(I I_{\dot{\gamma}}\right)$ in Equation 2.75 takes the form

$$
\eta=k_{1} \dot{\gamma}^{n-1}+k_{2} \dot{\gamma}^{-1}
$$

Which forms a constitutive equation similar to the Power law (Table 2.3) with the addition of a yield stress term.

\footnotetext{
${ }^{17}$ As the original reference from W.H. Herschel and R. Bulkley [21] is written in German the reader is alternatively directed to a textbook. This strategy of citing original non-English work and an accompanying English reference will be used elsewhere in this thesis without specific mention.
} 


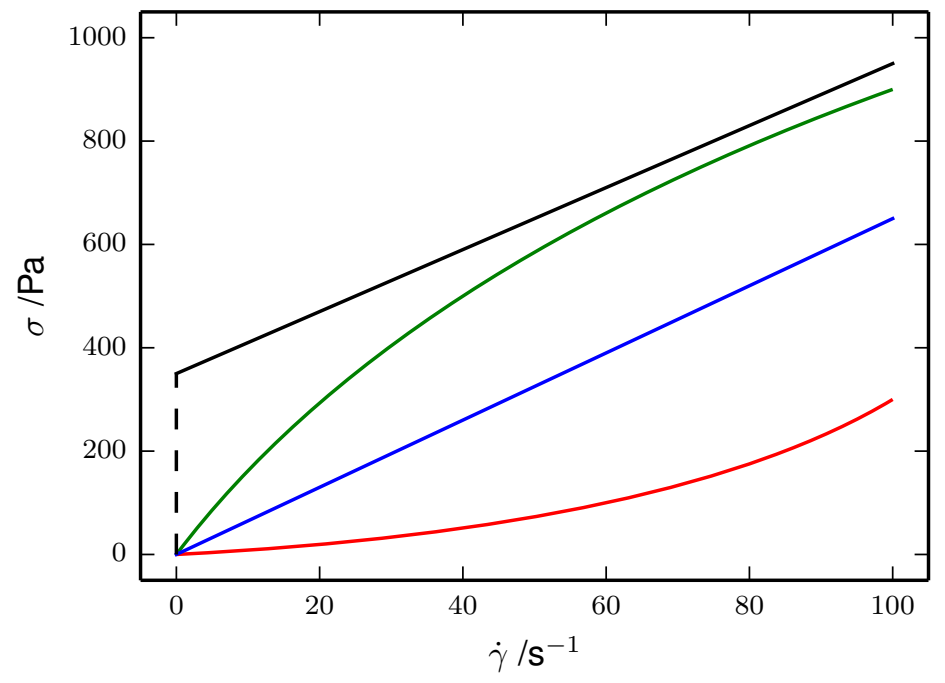

Figure 2.8: Depiction of the shear stress response as a function of shear rate for various classes of fluids: constant viscosity (blue), Bingham plastic behaviour (black), shearthickening (red) and shear-thinning (green).

While the concept of yield stress has been particularly useful for the characterisation of materials over a specific range of shear rates the existence of a true yield stress quantity has been questioned [22, 23]. The ability to measure yield stress appears to be directly related to available measurement techniques and further complicated by thixotropy, aging, slip, shear banding and stress localisation [24].

\subsubsection{Viscoelasticity}

In the previous sections materials were presented to have either elastic or viscous properties (Section 2.3.1 and 2.3.2, respectively). However, it has been suggested that all real materials simultaneously exhibit behaviour of both ideal liquids and ideal solids [6, pp. 37], a characteristic termed viscoelasticity. Early quantification of viscoelastic behaviour dates back to 1835 when W. Weber [25][5, pp. 2] observed silk threads under tension. Weber noted that when placed under tensile load the silk fibres immediately responded with elastic extension, but when left in tension they would slowly continue to elongate. In Figure 2.9 Weber's experiment (single step stress) is applied to various types of materials. Furthermore, Weber observed that when the applied stress was removed the threads would eventually recover their original length. At the time pure elastic or plastic deformation could not explain this behaviour. For a viscoelastic material under test, as demonstrated by Weber, the response will depend on the time scale over which measurements are made. 

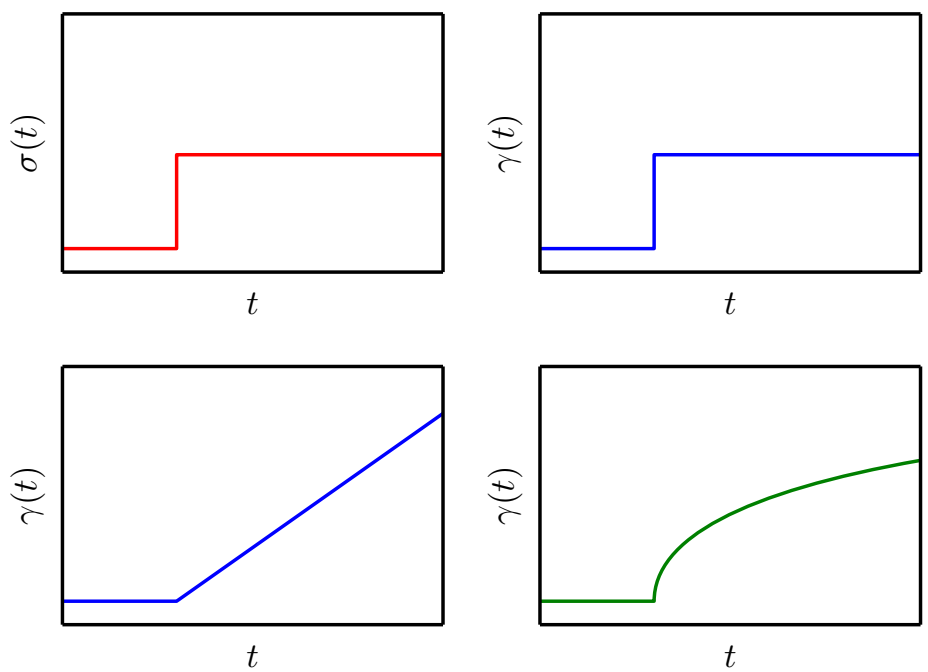

Figure 2.9: Material response to a hypothetical step stress experiment (actual experiments could not produce instantaneous change in applied stress). Single step stress input (top left); response of ideal elastic (Hookean) material (top right); ideal viscous (Newtonian) response (bottom left); viscoelastic material behaviour with initial jump in strain followed by increasing strain with time (bottom right).

To describe the ratio between the characteristic times for a material $\lambda^{18}$ to the observation time $t$, in 1964 M. Reiner [26] introduced the Deborah number $D e$

$$
D e=\frac{\lambda}{t} \text {. }
$$

In the limits, as the Deborah number approaches 0 , the material behaves like a perfect liquid (Newtonian); on the opposite end of the scale as $D e$ approaches $\infty$ the material can be described as an ideal solid (Hookean). In between the material is viscoelastic and names such as viscoelastic liquid and viscoelastic solid indicate how they are distributed across the spectrum (Figure 2.10).

An introduction to both linear and non-linear viscoelasticity follows but for a detailed treatment the reader is referred to the text by J.D. Ferry [27] or the collection of lecture notes from A.C. Pipkin [28].

\section{Linear Viscoelasticity}

The linear viscoelasticity regime describes the case in which the response (i.e. strain or stress) of a material varies linearly with the controlling input (i.e. stress or strain).

\footnotetext{
${ }^{18}$ In the Official symbols and nomenclature of The Society of Rheology [2] the Greek symbol $\tau$ with various subscript to describe relaxation times; however, to avoid confusion with components of the extra stress tensor, $\lambda$ will be used in this chapter to describe generic relaxation time.
} 


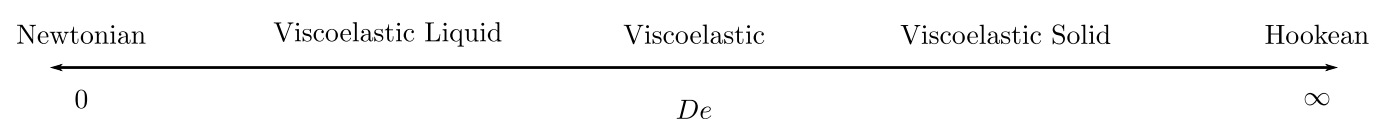

Figure 2.10: Number scale depicting the Deborah Number De (Equation 2.83) and corresponding material behaviours.

In previous sections, two forms of deformation have been discussed, uniaxial extension and simple shear. However, in the limit of small strains, this distinction is not important (a point which will be discussed later in the section).

Following the example of H.A. Barnes, J.F. Hutton and K. Walters [6, pp. 38], a general differential equation can be constructed in one dimension for linear viscoelasticity in simple shear

$$
\left(1+a_{1} \frac{\partial}{\partial t}+a_{2} \frac{\partial^{2}}{\partial t^{2}}+\cdots+a_{n} \frac{\partial^{n}}{\partial t^{n}}\right) \sigma_{12}=\left(b_{0}+b_{1} \frac{\partial}{\partial t}+b_{2} \frac{\partial^{2}}{\partial t^{2}}+\cdots+b_{n} \frac{\partial^{n}}{\partial t^{n}}\right) \gamma
$$

where the coefficients of the time derivatives are constant material properties (e.g. viscosity, shear modulus). To explore the utility of this differential equation, briefly consider two special cases. First if $b_{0}$ is the only non-zero coefficient, Equation 2.84 reduces to Hooke's law with $b_{0}$ as the shear modulus. Second if the $b_{1}$ term is the only non-zero parameter the result is Newton's equation where $b_{1}$ is viscosity.

Alternatively, if both $b_{0}$ and $b_{1}$ ( $G$ and $\eta$, respectively) were non-zero the result would be the so called Kelvin-Voigt model [29][5, pp. 3]

$$
\sigma_{12}=G \gamma+\eta \dot{\gamma}
$$

where the stress is a sum of an elastic stress $G \gamma$ and a viscous stress $\eta \dot{\gamma}$. Modelling these two components with mechanical analogues (a Hookean spring and Newtonian dashpot) the Kelvin-Voight model is depicted in Figure 2.11 where the two elements are in parallel.

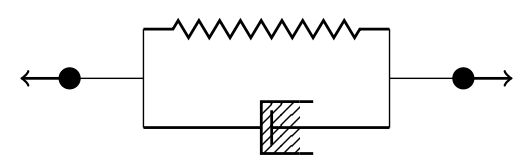

Figure 2.11: Mechanical representation of the Kelvin-Voigt model of viscoelasticity where the spring element represents the pure elastic contribution and the dashpot the viscous component.

Considering the mechanical configuration of the Kelvin-Voigt model a second viscoelastic model may be appropriate in which the spring and dashpot are placed in series. Today this configuration is referred to as the Maxwell model (Figure 2.12) [30]. 


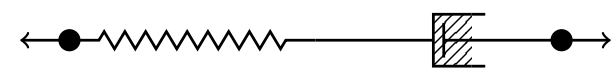

Figure 2.12: Mechanical representation of Maxwell model for viscoelasticity.

The differential form of the Maxwell model in one dimension is

$$
\sigma_{12}+\lambda \dot{\sigma}_{12}=\eta \dot{\gamma}
$$

and can be extended to three dimensions using tensor notation

$$
\boldsymbol{\sigma}+\lambda \dot{\boldsymbol{\sigma}}=\eta \dot{\gamma}
$$

where in either form, the term $\lambda$ is defined as the ratio between the viscosity of the Newtonian dashpot and the shear modulus of the Hookean spring $\frac{\eta}{G}$. This ratio carries units of time and is referred to as a relaxation time.

An alternative to forming viscoelastic constitutive equations through differential equations would be to use equivalent descriptions in integral form. In developing these relationships a history of successive small steps (i.e. strain or stress) will be summed over a discrete number and then extended to an integral of an arbitrary large number of steps. This approach is known as the Boltzmann Superposition Principle [31][28, pp. 10]. For this approach a time dependent relaxation modulus $G(t)$ will be used, defined as

$$
G(t)=\frac{\sigma(t)}{\gamma(t)} .
$$

As an illustration of the relaxation modulus $G(t)$ consider the hypothetical experiment in Figure 2.13 where a single step strain is applied to various materials. In the ideal case of a Hookean elastic solid the stress would remain constant as long as the strain was maintained. However, in the case of a Newtonian viscous fluid there would be an infinite stress during the instant the strain is applied. Of course for a real experiment the strain would be applied over some discrete time and the respective materials would behave appropriately. Finally, in the case of a viscoelastic material, once the strain has been applied the stress would decay with time. The relaxation modulus is a parameter which can be used to characterise this stress decay in a viscoelastic material. An analogous experiment was described in the introduction to this section (Figure 2.9) in which a single step stress was applied and the strain evolved as a function of time. In this case a different function, creep compliance $J(t)$, can be used to describe this behaviour

$$
J(t)=\frac{\gamma(t)}{\sigma(t)} .
$$

While there is certainly a relationship between these two material functions (i.e. $G(t)$ and $J(t))$ and the methods which follow could similarly be developed for creep compliance, primary focus will be given to the relaxation modulus. 

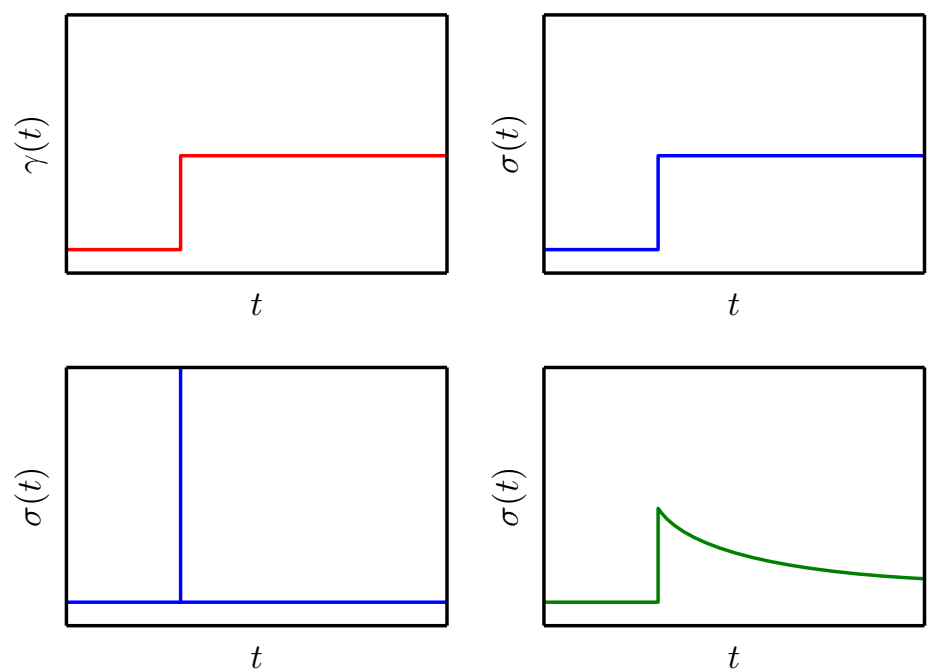

Figure 2.13: Ideal step strain experiment to illustrate the relaxation modulus of viscoelastic materials. Under a single step strain input (top left) the stress is shown as a function of time for an ideal elastic solid (top right), ideal viscous fluid (bottom left) and viscoelastic material (bottom right).

At the start of the section a statement was made that in the limit of small strain the exact form of deformation is not important; however, no specific evidence was given to support this claim. The relaxation modulus described above is a time dependent equivalent of the shear modulus which naturally arises from the scenario of simple shear. However, in discussing uniaxial extension for Neo-Hookean model Equation 2.40 showed that for small strains Young's modulus $E$ was three times the shear modulus $G$ and as such the shear modulus (and by extension the time dependent relaxation modulus) can be used to describe uniaxial extension in the limit of small strains.

Considering the single strain step experiment presented in Figure 2.13 where a single strain $\Delta \gamma_{o}$ is applied at some time $t_{o}$. Equation 2.88 can be rewritten as

$$
\sigma(t)=\Delta \gamma_{o} G\left(t-t_{o}\right) .
$$

Here the relaxation modulus is dependent on the time lag between the current time $t$ and the time when the strain was applied $t_{o}$; the strain input for this experiment is also depicted in the top panel of Figure 2.14. If some short time later a second strain step is applied the stress history would be the addition of these two individual events,

$$
\sigma(t)=\Delta \gamma_{1} G\left(t-t_{1}\right)+\Delta \gamma_{2} G\left(t-t_{2}\right)
$$

a scenario which is depicted in the centre panel of Figure 2.14. As more successive strains are applied the Boltzmann Superposition Principle can be used to represent the 
stress history as a summation of past events

$$
\sigma(t)=\sum_{1}^{N} \Delta \gamma_{N} G\left(t-t_{N}\right)
$$

As the number of steps increase where each contributes some infinitesimal strain an integral can be used to describe the stress history over all previous time (bottom panel Figure 2.14)

$$
\sigma(t)=\int_{-\infty}^{t} G\left(t-t^{\prime}\right) \dot{\gamma}\left(t^{\prime}\right) d t^{\prime}
$$

where the $d \gamma\left(t^{\prime}\right)$ term in the integral has been replaced by $\dot{\gamma}\left(t^{\prime}\right) d t^{\prime}$. Commonly the elapsed time $t-t^{\prime}$ is replaced by a single variable $s$. With this substitution and change of the limits of integration a general constitutive equation for linear viscoelasticity can be written

$$
\sigma(t)=\int_{o}^{\infty} G(s) \dot{\gamma}(t-s) d s
$$

The integral in Equation 2.93 defines the stress response as a function of arbitrary strain input with use of the relaxation modulus; however, it is still not clear what form $G(t)$ will take. Assuming an exponential decay with a relaxation time $\lambda$ the relaxation modulus could take the form

$$
G(t)=G_{o} e^{-t / \lambda}
$$

which substituted into Equation 2.93 is the one dimensional Maxwell model in integral form (equivalent to Equation 2.86)

$$
\sigma(t)=\int_{0}^{\infty} G_{o} e^{-s / \lambda} \dot{\gamma}(t-s) d s .
$$

In establishing both the integral and differential forms of the Maxwell model above, it was assumed that a single relaxation time $\lambda$ was sufficient. In real materials these relaxation times may be directly related to various molecular processes and a single characteristic time may not accurately describe the complexity of the material. A generalised Maxwell model can be constructed by allowing $N$ numbers of Maxwell elements to co-exist in a material; mechanically, this is depicted in Figure 2.15. In this case the relaxation modulus is considered a sum of $N$ decays

$$
G(t)=\sum_{i=1}^{N} G_{o_{i}} e^{-t / \lambda_{i}}
$$

which substituted into Equation 2.93 defines the Generalised Maxwell model in one dimension

$$
\sigma(t)=\int_{0}^{\infty} \sum_{i=1}^{N} G_{o i} e^{-s \lambda_{i}} \dot{\gamma}(t-s) d s .
$$



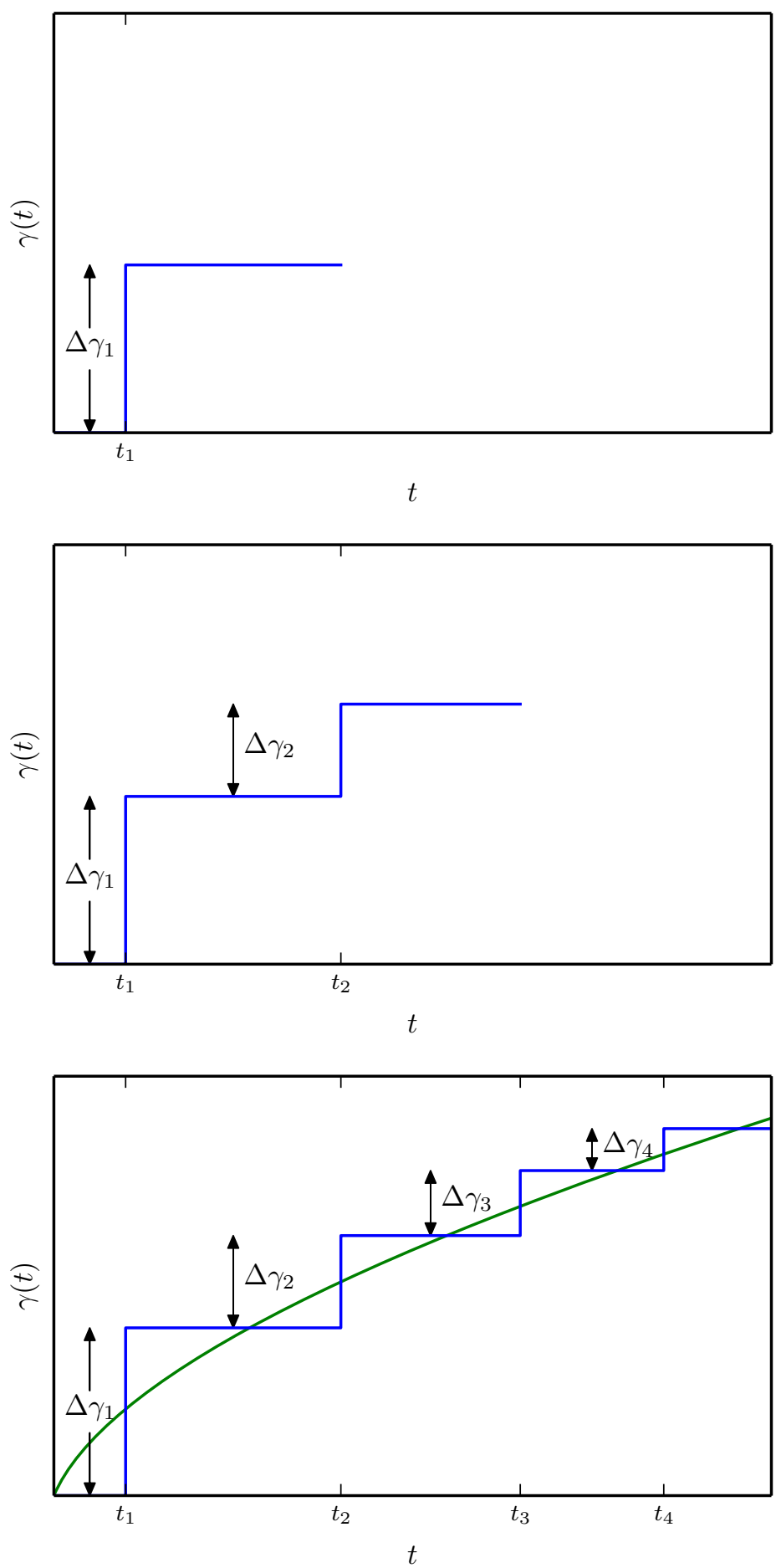

Figure 2.14: Illustration of the Boltzmann Superposition Principle for viscoelastic materials. All plots show the strain history of a sample under test; single step strain $\Delta \gamma$ (top), two discrete strain steps (middle) and many steps can be treated as a sum of numerous steps (bottom). 


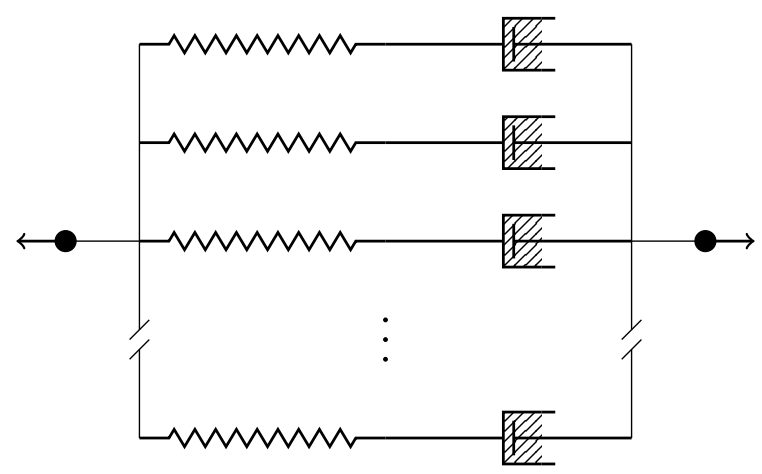

Figure 2.15: Mechanical representation of Generalised Maxwell model for viscoelasticity.

Furthermore, the discrete set of relaxation times in Equation 2.98 can be replaced by a continuous function $F(\lambda)$

$$
G(t)=\int_{0}^{\infty} F(\lambda) e^{-t / \lambda} d \lambda .
$$

Typically an alternative distribution function, the relaxation spectrum $H(\lambda)$, is used to represent the spectrum of relaxation frequencies on a logarithmic basis

$$
G(t)=\int_{0}^{\infty} H(\lambda) e^{-t / \lambda} \frac{d \lambda}{\lambda}
$$

Similar to describing the relaxation modulus $G(t)$ with the relaxation spectrum $H(\lambda)$, it can be shown that creep compliance is the integral of the retardation spectrum $L(\lambda)$. Specific forms of these spectra and their interconversions will not be explored but are discussed in detail in the textbook from J.D. Ferry [27].

Constitutive equations for viscoelastic materials are typically written in terms of either the relaxation modulus or creep compliance because these material functions can easily be measured. However, in the case of very short relaxation processes a simple step experiment may not be sufficient. Instead sinusoidal strain waveforms are used. For the remainder of this section the basics of oscillatory rheology will be discussed.

Consider an oscillating strain input $\gamma(t)$

$$
\gamma(t)=\gamma_{o} \sin (\omega t)
$$

where $\gamma_{o}$ is the strain amplitude and $\omega$ is the angular frequency. Based on this strain, the strain rate would oscillate as

$$
\dot{\gamma}(t)=\gamma_{o} \omega \cos (\omega t)
$$

where the strain and strain rate would be exactly $\frac{\pi}{2}$ out of phase. In the case of an ideal solid, described by Hooke's law, the stress $\sigma(t)$ would be exactly in phase with 
the strain $\gamma(t)$; while a fluid with Newtonian behaviour would be exactly in phase with the strain rate $\dot{\gamma}(t)$. A real (viscoelastic) material would have a stress response with a phase shift $\delta$ such that $0 \leq \delta \leq \frac{\pi}{2}$. A strain (strain rate) input and stress response of a viscoelastic material is demonstrated in Figure 2.16.

The stress response to the sinusoidal strain input is described by

$$
\sigma(t)=\gamma_{o}\left|G^{*}\right| \sin (\omega t+\delta)
$$

where $G^{*}$ is the complex modulus and is composed of both a real and imaginary component

$$
G^{*}=G^{\prime}+i G^{\prime \prime}
$$

The real part $G^{\prime}$, called the storage modulus, represents the elastic behaviour while the imaginary component $G^{\prime \prime}$, referred to as the loss modulus, characterises the viscous behaviour. The stress from either component can be determined using

$$
\begin{gathered}
\sigma_{o}^{\prime}=\gamma_{o} G^{\prime} \\
\sigma_{o}^{\prime \prime}=\gamma_{o} G^{\prime \prime} .
\end{gathered}
$$

In the linear viscoelastic regime the complex modulus $G^{*}$ does not depend on strain amplitude $\gamma_{o}$ and is purely a function of angular frequency $\omega$.

\section{Nonlinear Viscoelasticity}

In the previous section linear viscoelasticity was described as the regime in which a material's response (e.g. stress, strain) is linearly proportional to input functions (e.g. strain, strain rate, stress), an assumption which is reasonable in the limit of small strains. Furthermore, a general theory of viscoelasticity Equation 2.94 and subsequently the Maxwell model of linear viscoelasticity (Equation 2.86 or 2.96) were developed. Written in three dimensions, with the rate of strain tensor, the Maxwell model could be applied to the case of simple shear deformations where the model would not predict normal stress differences. However, true materials (e.g. polymeric solutions) frequently exhibit effects associated with non-zero normal stress differences, with examples found in early work from K. Weissenberg [32]. To model both real materials and real-world processes a nonlinear response must be considered. Beginning with early work in the 1930s numerous constitutive equations have been proposed. Due to both the quantity and complexity of these models these concepts will not be explored in detail and alternatively the reader is directed towards an excellent introduction (with further references) in the text from C.W. Macosko [1, chap.4 and ref. within]. 

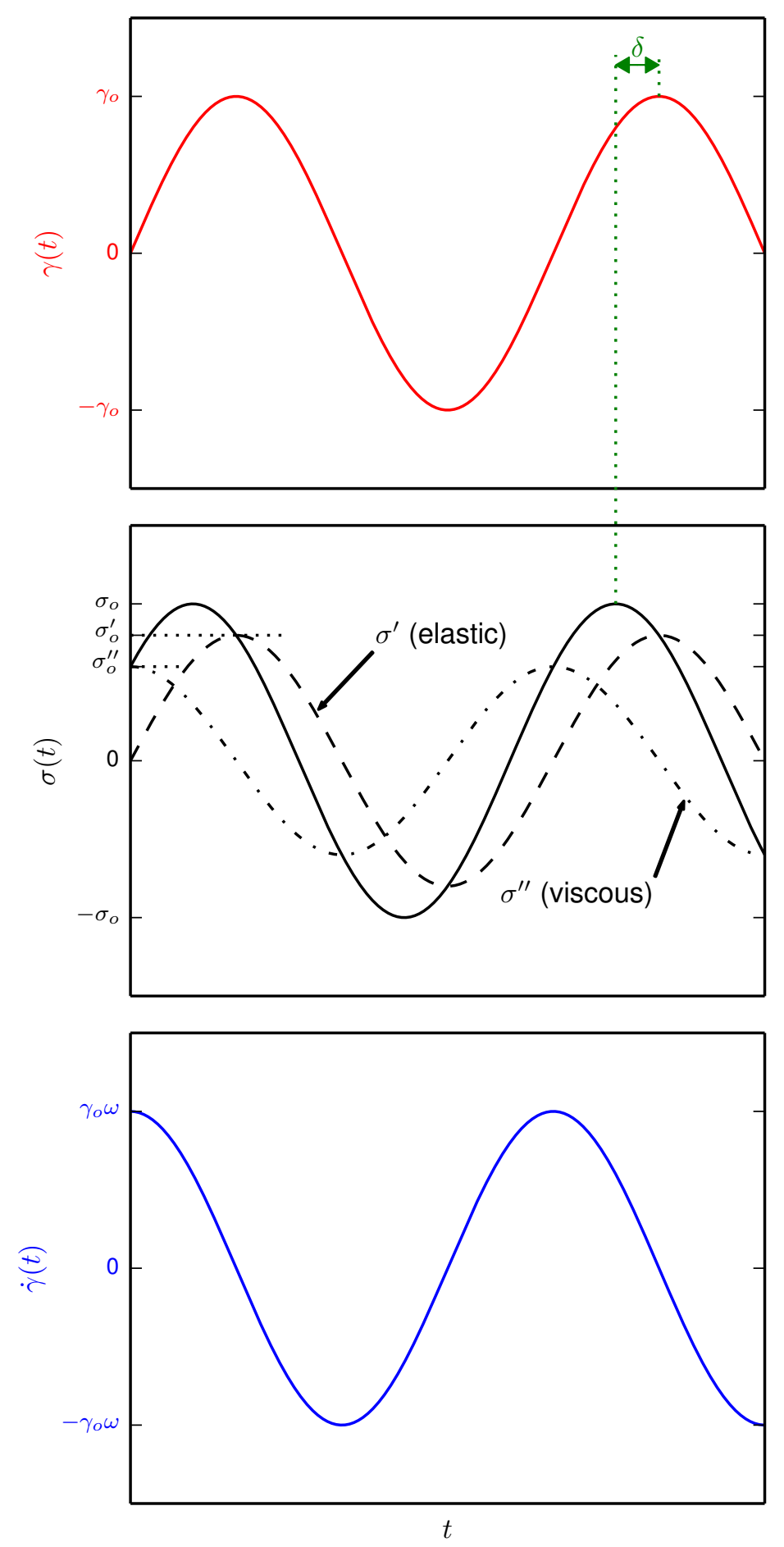

Figure 2.16: Illustration of an imposed oscillatory strain waveform and the stress response of a viscoelastic material. The top panel illustrates the sinusoidal input strain waveform which could alternatively be described by a sinusoidal shear rate waveform (bottom panel) which is phase shifted from the strain by $\frac{\pi}{2}$. The middle panel illustrates the stress response (solid line) which can be described as the sum of an elastic component (dashed line) and a solid component (dash dot line). 


\subsection{Measurements}

In the previous sections various models and subsequent constitutive equations were developed to describe material behaviour when subjected to deformation. Practically, these models would be used to predict how an actual material would respond in realworld scenarios. However, before a model can be ascribed to a new material, its behaviour must be observed when precisely controlled stresses or deformations are applied. This is the essence of rheometry, which is the art of measuring rheological quantities. A broad introduction and practical treatment of the subject can be found in the text from C.W. Macosko [1, part II].

Today there are a number of commercial suppliers of industrial and laboratory grade rheological instruments. In addition, the rheology community has generated a great number of homemade devices over the past one hundred years. In general, these instruments fall mainly into two categories: shear rheology and extensional rheology.

Extensional rheometers are particularly relevant for high viscosity materials. These instruments are extremely useful for industry as numerous polymer processing operations (e.g. injection molding, film blowing or fiber spinning) involve extensional deformation.

Shear rheology can further be subdivided into drag flow and pressure driven flow. Pressure driven measurements include capillary rheology and slit rheology which can be operated in either a controlled drive pressure or controlled flow rate mode. Pressure driven flow rheology is useful for process line measurements where rheological parameters are used as controls in industrial environments. In the case of drag flow rheology materials are sheared between a moving and stationary boundary.

The experimental methods presented in this thesis focus primarily on rotational drag flow shear rheology which utilises symmetric geometries to produce continuous shear. For this reason the following section will focus on this topic. Extensional rheology combined with nuclear magnetic resonance will be discussed in Section 4.4.3 where a brief introduction to this class of measurements will be given.

\subsubsection{Rotational Drag Flow Rheometers}

The first rotary drag flow rheometer was introduced in 1890 by M. Couette [33][1, pp. 181] with commercially available devices appearing only two decades later [34]. Over the past one hundred years rotary rheometers have continuously evolved and are now a common tool in industry and academia. In addition to various experimental protocols (e.g. step, continuous, sinusoidal waveforms) and available shear geometries (discussed in the following section), modern rotary rheometers may also have provisions to measure normal stresses and for sophisticated sample temperature control [35][1, chap. 
8].

In the case of the rheometer built by Couette the angular velocity of one shear surface was controlled while a torque was calculated based on the angular deflection of the opposing surface (in this design a concentric cylinder geometry was used and will be discussed in the following section). For this and other controlled strain devices the strain (or strain rate) is the independent variable while the stress (calculated from torque) is the dependent variable. Strain input is typically provided by an electric motor with modern instruments utilising a DC motor operating with a position encoder in a closed loop feedback (a configuration referred to as a servomotor). Optical or capacitance encoders allow for both discrete steps (position control) or continuous steady shear (velocity control) including time dependent strain waveforms (e.g. sinusoidal). Early instruments utilised a torsion element with various angular deflection methods to measure torque. However, the requirement for angular displacement of the torsion element meant that in the case of step experiments (e.g. stress relaxation) the true strain experienced by the sample would be the imposed strain minus the deflection of the measurement device and additional transient errors would occur. Although the displacement of the torsion element could be reduced by introducing corrections to the control software a zero displacement torque measurement could be achieved with the use of a force rebalance device. A servo motor was used to eliminate the angular deflection across a torsion element and the torque measurement was determined from the current necessary to maintain this zero displacement. A modern strain controlled rheometer (utilising a force rebalance transducer) can be seen in Figure 2.17a. Additionally, a torque measurement on a strain controlled rheometer could be determined simply from the current required to maintain constant rotation (the same theory is used in the force rebalance transducer). Initially applied to DC motors this technique was later applied to devices with electrically commutated (or brushless DC) motors. One special case of a strain controlled rheometer is a viscometer which is limited to measuring the viscosity of a sample in steady shear flows.

Controlled stress devices are the inverse of the controlled strain rheometer described above with the independent variable being shear stress and the dependent variable being the strain (or strain rate) [34]. Characterising the strain (or strain rate) is relatively straight forward as angular displacements (or angular frequencies) can be measured with the same elements used in the control routine for strain controlled rheometers (e.g. capacitance or optical encoders). Controlling the torque input is a greater engineering challenge. For early devices a weight attached to a string, coiled around a pulley, would be dropped creating a torque on the central shaft of the pulley during free fall. Of course practical considerations (e.g. drop length) limited the utility of such devices. In the 1960s J. Deer modified the air bearing of a strain controlled rheometer 


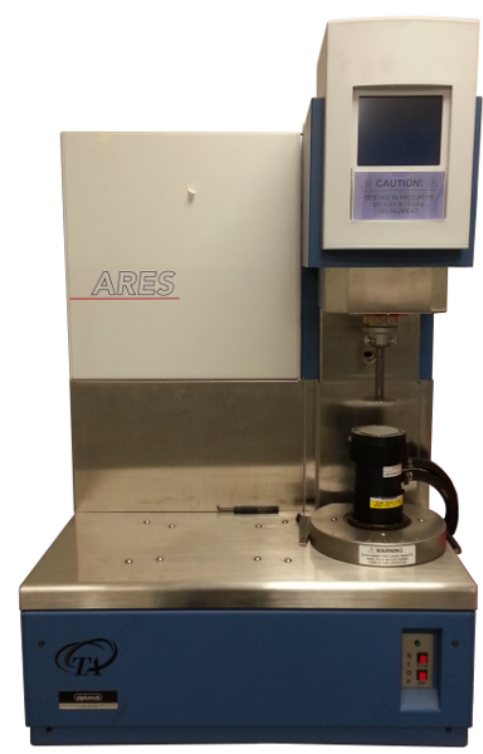

(a)

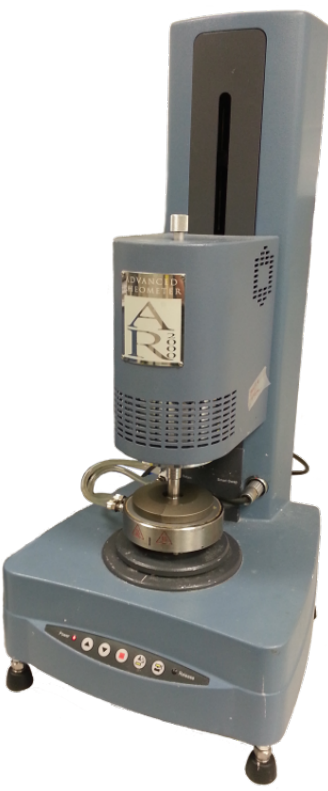

(b)

Figure 2.17: Examples of commercial rheometers: (a) TA Instruments ARES strain controlled rheometer and (b) TA Instruments AR 2000 stress controlled rheometer.

such that continuous controlled stress was possible [36]. With time the air turbine drive system was replaced by an asynchronous AC motor (referred to as drag-cup motor). Figure $2.17 \mathrm{~b}$ shows a commercial stress controlled rheometer with a drag-cup motor. Modern stress controlled devices have the added benefit of pseudo-strain control in which a feedback loop between the shaft position (or speed) and motor voltage can be used to generate an input strain.

\subsubsection{Rotational Drag Flow Geometries}

Practically, true simple shear deformation is difficult to realise for viscous and viscoelastic fluids due to sample containment and edge effects. Furthermore, physical limits on the length of the geometry would not only be a substantial engineering challenge but also limit the possible experiment time and range of obtainable shear rates. As an alternative, concentrically symmetric geometries are frequently used for continuous steady shear, including: 1) concentric cylinders, 2) cone and plate and 3) parallel disks. However, due to deviations from the ideal case of infinite sliding plates each set-up has specific benefits and disadvantages which will be discussed in the following sections. 


\section{Concentric Cylinders}

In the case of the concentric cylinder geometry a sample fills the annulus between two coaxial cylinders (Figure 2.18). Shear is produced from relative motion between the surfaces of the cylinders in contact with the fluid. In the case of the strain controlled rheometer designed by M. Couette, the outer cylinder (cup) of the geometry was rotated [33][1, pp. 181]. The opposite strategy was employed in the case of the stress controlled rheometer designed by G. Searle that utilised a geometry where the inner cylinder (bob) was rotated [37]. Both measurement strategies (illustrated in Figure 2.19) are still used today; rotating the outer cup has the advantage of stabilising secondary flows at high shear rates [38] while rotating the bob is a convenient option for multipurpose stress controlled rheometers.

In the case of an ideal concentric cylinder arrangement (cylindrical symmetry and no eccentricity), assuming steady laminar flow in the azimuthal direction $\frac{\partial v_{\theta}}{\partial t}=0$ and no secondary flow $v_{r}=v_{z}=0$, the equations of motion for a viscous fluid written in cylindrical coordinates (Appendix A.2) can be reduced to

$$
\begin{array}{ll}
r \text {-component : } & -\rho \frac{v_{\theta}^{2}}{r}=\frac{1}{r} \frac{\partial}{\partial r}\left(r \tau_{r r}\right)-\frac{\tau_{\theta \theta}}{r} \\
\theta \text {-component : } & 0=\frac{\partial}{\partial r}\left(r^{2} \tau_{r \theta}\right) \\
z \text {-component : } & 0=\frac{\partial}{\partial r}\left(r \tau_{r z}\right)+\rho g_{z}
\end{array}
$$

where the geometry has been aligned such that the only component from gravity acts in the $z$ direction. Normal stresses can be determined from Equation 2.106a, shear stress from 2.106b and hydostatic pressure from 2.106c. Assuming no-slip at walls (i.e. no discontinuities between the geometry and fluid velocity across the solid-fluid interfaces), the boundary conditions for a Searle device can be defined as $v_{\theta}\left(r_{\mathrm{i}}\right)=\Omega_{\mathrm{i}} r_{\mathrm{i}}$ and $v_{\theta}\left(r_{\mathrm{o}}\right)=0$ where $\Omega_{\mathrm{i}}$ is the angular frequency of the inner cylinder.

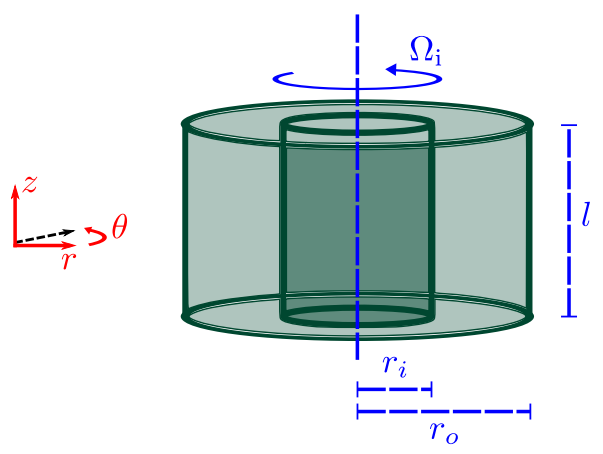

Figure 2.18: Simple schematic of a concentric cylinder (cylinderical Couette) shear geometry. 


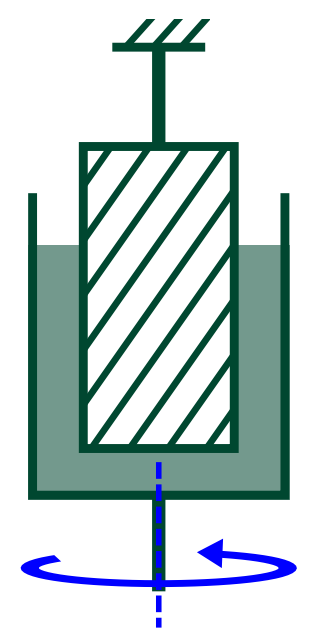

(a) Couette measurement mode

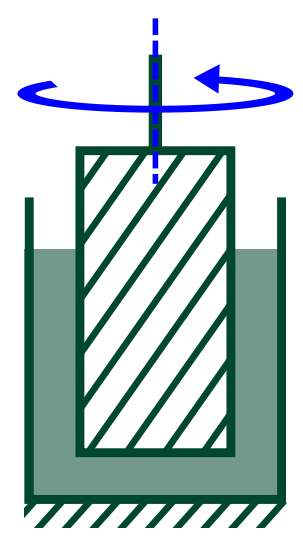

(b) Searle measurement mode

Figure 2.19: Measurement strategies for viscometer and rheometer instruments.

Integrating both sides of Equation 2.106b with respect to $r$ yields

$$
\sigma_{r \theta}(r)=\frac{c}{r^{2}}
$$

where the viscous stress component $\tau_{r \theta}$ has been replaced by an equivalent term, the total stress component $\sigma_{r \theta}$. The constant of integration $c$ can be calculated from a torque balance. To match the experimental methods used in this thesis torque will be measured at the inner wall. For this scenario, the torque balance would be

$$
M\left(r_{\mathrm{i}}\right)=\left(2 \pi r_{\mathrm{i}} l\right)\left(r_{\mathrm{i}}\right)\left(\frac{c}{r_{\mathrm{i}}^{2}}\right)
$$

where the three terms are the surface area, lever arm and shear stress at $r=r_{\mathrm{i}}$, respectively. Solving for $c$ and substituting the result into Equation 2.107 gives the shear stress as a function of measurable quantity $M\left(r_{\mathrm{i}}\right)$

$$
\sigma_{r \theta}\left(r_{\mathrm{i}}\right)=\frac{M\left(r_{\mathrm{i}}\right)}{2 \pi r_{\mathrm{i}}^{2} l}
$$

Torque $M$ is the physical quantity either controlled or measured when the concentric cylinder geometry is used on a rheometer. However, as illustrated in Figure 2.19, there are additional drag artefacts from the bottom of the bob which would not be treated in the equations above. In the original design from M. Couette, the solution was to disconnect the central region of the bob from the top and bottom. By only connecting the torque sensor to the central region these end effects were eliminated. However, this complicates the design and alternative methods will be discussed at the end of this section. 
Looking back at Equation 2.107 it is evident that shear stress varies with position $r$. The ratio of shear stress at the inner wall to the outer wall becomes

$$
\frac{\sigma_{r \theta}\left(r_{\mathrm{i}}\right)}{\sigma_{r \theta}\left(r_{\mathrm{o}}\right)}=\frac{r_{\mathrm{o}}^{2}}{r_{\mathrm{i}}^{2}}
$$

In the case of Newtonian behaviour (constant viscosity) the ratio of the shear rate between the two boundaries is

$$
\frac{\dot{\gamma}\left(r_{\mathrm{i}}\right)}{\dot{\gamma}\left(r_{\mathrm{o}}\right)}=\frac{r_{\mathrm{o}}^{2}}{r_{\mathrm{i}}^{2}}
$$

Indicating that the shear rate (and shear strain) is not constant between the two cylinders. However, as the curvature of the two walls becomes more similar the position dependence diminishes. In the extreme the two surfaces are infinitely parallel, returning to the case of simple shear.

From the definition of the rate of strain tensor $\dot{\gamma}$ (Equation 2.59) and using the components of deformation gradient tensor $\mathbf{L}$ (written in cylindrical coordinates [1, pp. 69]), the shear rate is

$$
\left|\dot{\gamma}_{r \theta}\right|=\dot{\gamma}=\left|\frac{1}{r} \frac{\partial}{\partial \theta}\left(v_{r}\right)-\frac{v_{\theta}}{r}+\frac{\partial}{\partial r}\left(v_{\theta}\right)\right|
$$

where the first term is zero (due to symmetry in $\theta$ ). The term $v_{\theta}$ describes the azimuthal velocity of the fluid and varies as a function of $r$; alternatively $v_{\theta}$ could be described by $\Omega r$ where $\Omega$ is the spatially dependent angular frequency of the fluid across the gap. As $\Omega$ varies as a function of $r$, the product rule is used to evaluate the third term in the shear rate description above, such that

$$
\dot{\gamma}=\left|r \frac{\partial}{\partial r}\left(\frac{v_{\theta}}{r}\right)\right|
$$

which describes, for the concentric cylinder geometry, how the shear rate $\dot{\gamma}$ varies as a function of position $r$. To evaluate the expression above, in the case of an incompressible fluid, the total stress tensor in the momentum equation (Equation 2.7) can be re-written in terms of dynamic viscosity $\eta$

$$
\boldsymbol{\sigma}=-p \mathbf{I}+\boldsymbol{\tau}=-p \mathbf{I}+\eta\left(\nabla \boldsymbol{v}+(\nabla \boldsymbol{v})^{T}\right) .
$$

With this modification, the equation of motion is referred to as the Navier-Stokes equation [9, sec. 4.1]. In this form, the $\theta$-component becomes

$$
0=\eta\left[\frac{\partial}{\partial r}\left(\frac{1}{r} \frac{\partial}{\partial r}\left(r v_{\theta}\right)\right)\right]
$$

Integrating twice and solving for velocity $v_{\theta}$,

$$
v_{\theta}(r)=c_{1} r+\frac{c_{2}}{r} .
$$


Using the two boundary conditions listed earlier the constants of integration $c_{1}$ and $c_{2}$ can be determined and used to solve for the azimuthal velocity $v_{\theta}$ as a function of position $r$

$$
v_{\theta}(r)=\frac{\Omega_{\mathrm{i}} r_{\mathrm{i}}^{2}}{r_{\mathrm{o}}^{2}-r_{\mathrm{i}}^{2}}\left(\frac{r_{\mathrm{o}}^{2}}{r}-r\right) .
$$

For the velocity profile equation listed above, if both sides are divided by $r$, this function can be substituted into the formula for shear rate (Equation 2.113)

$$
\dot{\gamma}(r)=\left|r \frac{\partial}{\partial r}\left[\frac{\Omega_{\mathrm{i}} r_{\mathrm{i}}^{2}}{r_{\mathrm{o}}^{2}-r_{\mathrm{i}}^{2}}\left(\frac{r_{\mathrm{o}}^{2}}{r^{2}}-1\right)\right]\right|
$$

and after taking the absolute value of the derivative

$$
\dot{\gamma}(r)=\frac{2 \Omega_{\mathrm{i}}}{r^{2}}\left(\frac{r_{\mathrm{i}}^{2} r_{\mathrm{o}}^{2}}{r_{\mathrm{o}}^{2}-r_{\mathrm{i}}^{2}}\right)
$$

In which the $\dot{\gamma}(r) \propto \frac{1}{r^{2}}$ behaviour is evident. In the case that $r_{\mathrm{o}} \approx r_{\mathrm{i}}$ any position across the annulus could be represented by a average value $\bar{r}$

$$
\bar{r}=\frac{r_{\mathrm{o}}+r_{\mathrm{i}}}{2} .
$$

By expanding the $r_{\mathrm{o}}^{2}-r_{\mathrm{i}}^{2}$ term in Equation 2.119 to $\left(r_{\mathrm{o}}-r_{\mathrm{i}}\right)\left(r_{\mathrm{o}}+r_{\mathrm{i}}\right)$ and assuming $r_{\mathrm{o}} \approx r_{\mathrm{i}} \approx \bar{r}$, simple shear behaviour is recovered

$$
\dot{\gamma}=\frac{\Omega_{\mathrm{i}} \bar{r}}{r_{\mathrm{o}}-r_{\mathrm{i}}} .
$$

In the case that the ratio between the curvature of the two cylinders $\kappa=\frac{r_{1}}{r_{\mathrm{o}}}$ is greater than 0.99 , curvature can be neglected and the equation above for shear rate in the case of simple shear can be used [1, pp. 191].

In the work above determining the form of the shear rate was straightforward based on the assumption of an incompressible fluid with Newtonian behaviour. An alternative derivation could be used where the shear rate function is defined in terms of shear stress and subsequently developed for a generic fluid [1, pp. 191-195]. This function can then be used to convert angular frequency to a true shear rate. However, for simplicity, some practitioners choose to report an applied shear rate $\dot{\gamma}_{a}$

$$
\dot{\gamma}_{a}=\frac{\Omega_{\mathrm{i}} r_{\mathrm{i}}}{r_{\mathrm{o}}-r_{\mathrm{i}}} .
$$

In formulating the equations of motion for the concentric cylinder geometry a noslip condition was assumed at the solid-fluid interfaces. For actual experiments this may not hold due to the presence of true or apparent slip [39]. Without local velocity measurements it can be difficult to detect slip. If multiple measurements are made on a series of concentric cylinder geometries with varying dimensions it may be possible 
to correct for slip [40]. If slip is a genuine concern other geometries (i.e. parallel disks) may be more appropriate.

In the opening paragraph of this section there was a reference to the work of G. I. Taylor [38] who explored flow instabilities (Taylor vortices) at high angular frequencies. Using the Taylor number Ta, a criterion for inertial instabilities (in the case of a rotating inner cylinder) is given by [1]

$$
\mathrm{Ta}=\frac{\rho^{2} \Omega_{\mathrm{i}}^{2}\left(\mathrm{r}_{\mathrm{o}}-\mathrm{r}_{\mathrm{i}}\right)^{3} \mathrm{r}_{\mathrm{i}}}{\eta(\dot{\gamma})^{2}}<3400 .
$$

Although this criterion was developed in the case of Newtonian behaviour and a narrow gap $(\kappa>0.99)$ it may be used generally to estimate stability in more complicated scenarios. In other work [e.g. 41] inertial instabilities are described by the term $\Sigma_{i}$ defined as

$$
\Sigma_{i}=\sqrt{\left(\frac{1-\kappa}{\kappa}\right)} \operatorname{Re}
$$

where $\operatorname{Re}$ is the Reynolds number, $\operatorname{Re}=\frac{d^{2} \dot{\gamma}}{\nu}$, in which $\nu$ is the kinematic viscosity. Using the definitions described in this chapter it can be shown that the Taylor number and $\Sigma_{i}$ are related such that $\mathrm{Ta}=\Sigma_{\mathrm{i}}^{2}$ for the case of a narrow gap. Furthermore, for concentric cylinders with a rotating inner wall (frequently referred to as Taylor-Couette or TC devices in the literature), elastic instabilities were first described for dilute polymer solutions by R. G. Larson, E. S. G. Shaqfeh and S. J. Muller [42]. This elastic instability can be described by

$$
\Sigma_{e}=\sqrt{\left(\frac{1-\kappa}{\kappa}\right)} \mathrm{Wi}
$$

where $\mathrm{Wi}=\lambda \dot{\gamma}^{19}$ is the Weissenberg number [41]. Using Equations 2.124 and 2.125 it has been suggested that flow becomes unstable when either term exceeds a critical coefficient on the order of unity [41].

Earlier in this section ends effects were mentioned briefly; today there are two common strategies used to account for flow underneath the rotor of the concentric cylinder: 1) machine a recessed pocket on the bottom of the bob which traps air when immersed in the sample fluid, ultimately isolating the bottom of the rotor or 2) taper the end to a cone (with appropriate angle) and account for the addition torque components when converting a measured torque to a shear stress. Considering the second option the formula for determining shear stress will now be derived. In this derivation it will be assumed that the concentric cylinder validates the narrow gap approximation $(\kappa>0.99)$; in this case the shear rate and shear stress are described by Equations 2.122 and 2.109,

\footnotetext{
${ }^{19}$ As mentioned previously, $\lambda$ is used in this chapter as a relaxation time while in other sources it is common for $\tau$ to be used for this purpose.
} 
respectively. The cone on the bottom of the rotor can be treated as a cone and plate geometry where the shear rate and shear stress are described by Equations 2.132 and 2.131 , respectively (these relationships will be discussed in the following section). The measured torque for an actual concentric cylinder geometry can be treated as a combination of torques from an ideal concentric cylinder and ideal cone and plate

$$
M_{\mathrm{t}}=M_{\mathrm{cc}}+M_{\mathrm{cp}}
$$

where $M_{\mathrm{t}}, M_{\mathrm{cc}}$ and $M_{\mathrm{cp}}$ are the measured torque, torque from an ideal concentric cylinder and torque from an ideal cone and plate, respectively. Using Equations 2.109 and 2.131 the measured torque could further be defined as

$$
M_{\mathrm{t}}=2 \pi r_{\mathrm{i}}^{2} l \sigma_{\mathrm{cc}}+\frac{2}{3} \pi r_{\mathrm{i}}^{3} \sigma_{\mathrm{cp}}
$$

where $\sigma_{\mathrm{cc}}$ and $\sigma_{\mathrm{cp}}$ are the shear stresses for the two geometries. In the event that the applied shear rates for the two geometries are matched, regardless of the underlying dynamics of the fluid, these shear stresses are the same $\left(\sigma_{\mathrm{cc}}=\sigma_{\mathrm{cp}}\right)$. Using Equations 2.122 and 2.132 the geometric criterion for equal shear rates is

$$
\beta=\frac{r_{\mathrm{o}}-r_{\mathrm{i}}}{r_{\mathrm{i}}} .
$$

Factoring out the shear stress in Equation 2.127

$$
M_{\mathrm{t}}=\left(2 \pi r_{\mathrm{i}}^{2} l+\frac{2}{3} \pi r_{\mathrm{i}}^{3}\right) \sigma_{12}
$$

and solving for the shear stress

$$
\sigma_{12}=\frac{M_{\mathrm{t}}}{2 \pi r_{\mathrm{i}}^{2} l\left(1+\frac{r_{\mathrm{i}}}{3 l}\right)} .
$$

Comparing the shear stress equation adjusted for end effects (Equation 2.130) to the ideal form (Equation 2.109) the correction factor in the denominator is clear. Furthermore, in the event that the immersion length $l$ is much larger than the radius of the rotor $r_{\mathrm{i}}$ the end effects can be ignored.

Normal stresses can be difficult to measure in the concentric cylinder geometry and if this data is necessary a more appropriate shear geometry would be the cone and plate. A summary of the characteristics of the concentric cylinder shear device can be found in Table 2.4 .

\section{Cone and Plate}

Originally introduced by M. Money and R. H. Ewart [43], the cone and plate configuration is well known for generating homogeneous deformation and is convenient for 


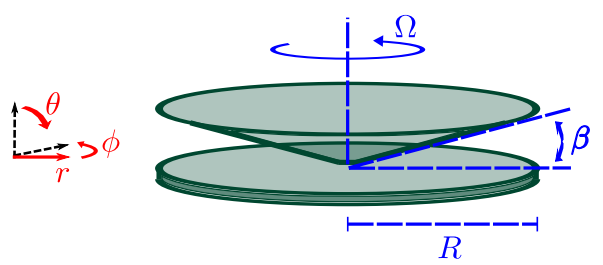

Figure 2.20: Simple schematic of a cone and plate shear geometry.

measuring normal stress. A general schematic of the geometry can be seen in Figure 2.20. Practically, the angle $\beta$ is limited to $<0.1$ radians $\left(\approx 6^{\circ}\right)$ such that small angle approximations can be used in deriving the equations for shear stress and shear rate. Following the procedure demonstrated for the concentric cylinder geometry and utilising spherical coordinates shear stress can be calculated from a measured torque $M$ using

$$
\sigma_{\theta \phi}=\frac{3 M}{2 \pi R^{3}}
$$

where $R$ is the radius of the cone (Figure 2.20). Furthermore, the shear rate is defined as

$$
\dot{\gamma}=\frac{\Omega}{\beta} .
$$

Thus the shear rate is not dependent on position and the deformation is uniform through the sample ${ }^{20}$.

As mentioned before the arrangement of the cone and plate allows for determination of the first normal stress difference $N_{1}$ by measuring the total thrust force along the axis of rotation [6, sec. 4.4.1]. The ability to measure thrust force is a common feature of laboratory rheometers where a force rebalance transducer (discussed previously in Section 2.4.1) is used to maintain the spacing between the two surfaces. To prevent normal forces from particles within the sample the tip of the cone $(\approx 50 \mu \mathrm{m})$ is commonly truncated.

\section{Parallel Disks}

The first parallel disks (plate and plate) configuration was used in an instrument designed by M.Mooney to test unvulcanised rubber [44]. A simple schematic and important dimensions for the parallel disks geometry can be seen in Figure 2.21.

Unlike the cone and plate geometry the deformation from the parallel disks is not homogeneous and the shear rate (as in the case of the concentric cylinders) varies with position $r$

$$
\dot{\gamma}(r)=\frac{r \Omega}{h}
$$

\footnotetext{
${ }^{20}$ Worked derivation available [1, pp. 205-208].
} 


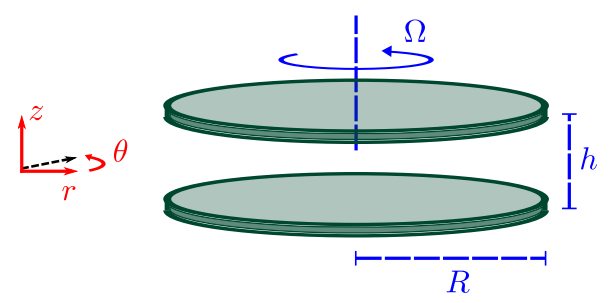

Figure 2.21: Simple schematic of a parallel disks (plate and plate) shear geometry

where it has been assumed there is no slip at the boundaries, steady flow in the azimuthal direction and no secondary flows. Furthermore, the shear stress is defined as

$$
\sigma_{r \theta}=\frac{M}{2 \pi R^{3}}\left(3+\frac{d \ln M}{d \ln \dot{\gamma}_{\mathrm{R}}}\right)
$$

where $\dot{\gamma}_{R}$ is the shear rate at the edge of the geometry ${ }^{21}$.

For the parallel disks arrangement, because the shear rate varies with position, measurements of the total thrust give the difference between $N_{1}$ and $N_{2}$. Thus, if the first normal stress difference $N_{1}$ is known (e.g. from cone and plate measurements), the second normal stress difference $N_{2}$ can be determined.

A significant advantage of the parallel disks arrangement is the ability to easily change the geometry for an experiment by varying the distance $h$ between the two plates. Successive experiments at different heights can be used to detect wall slip and in the case of Newtonian behaviour slip corrections are possible [40].

\subsection{Conclusion}

In this chapter fundamental concepts from continuum mechanics were introduced including mathematical tools (e.g. the total stress tensor $\boldsymbol{\sigma}$ and the rate of deformation tensor $\dot{\gamma}$ including their origin and application to basic forms of deformation, i.e. uniaxial extension and simple shear). These concepts were then used to generate constitutive equations for elastic solids, viscous liquids and viscoelastic materials. It was mentioned that most everyday items are some form of a viscoelastic material, having properties of both a pure liquid and true solid. A key motivation in the field of rheology is to develop material descriptions that account for one of more of the four key phenomena that can not be explained from Hooke's Law or Newton's viscosity, including:

1. Shear rate dependent viscosity (e.g. shear thinning or shear thickening)

2. Normal stresses in steady flows

\footnotetext{
${ }^{21}$ Worked derivation available [1, pp. 217-220].
} 


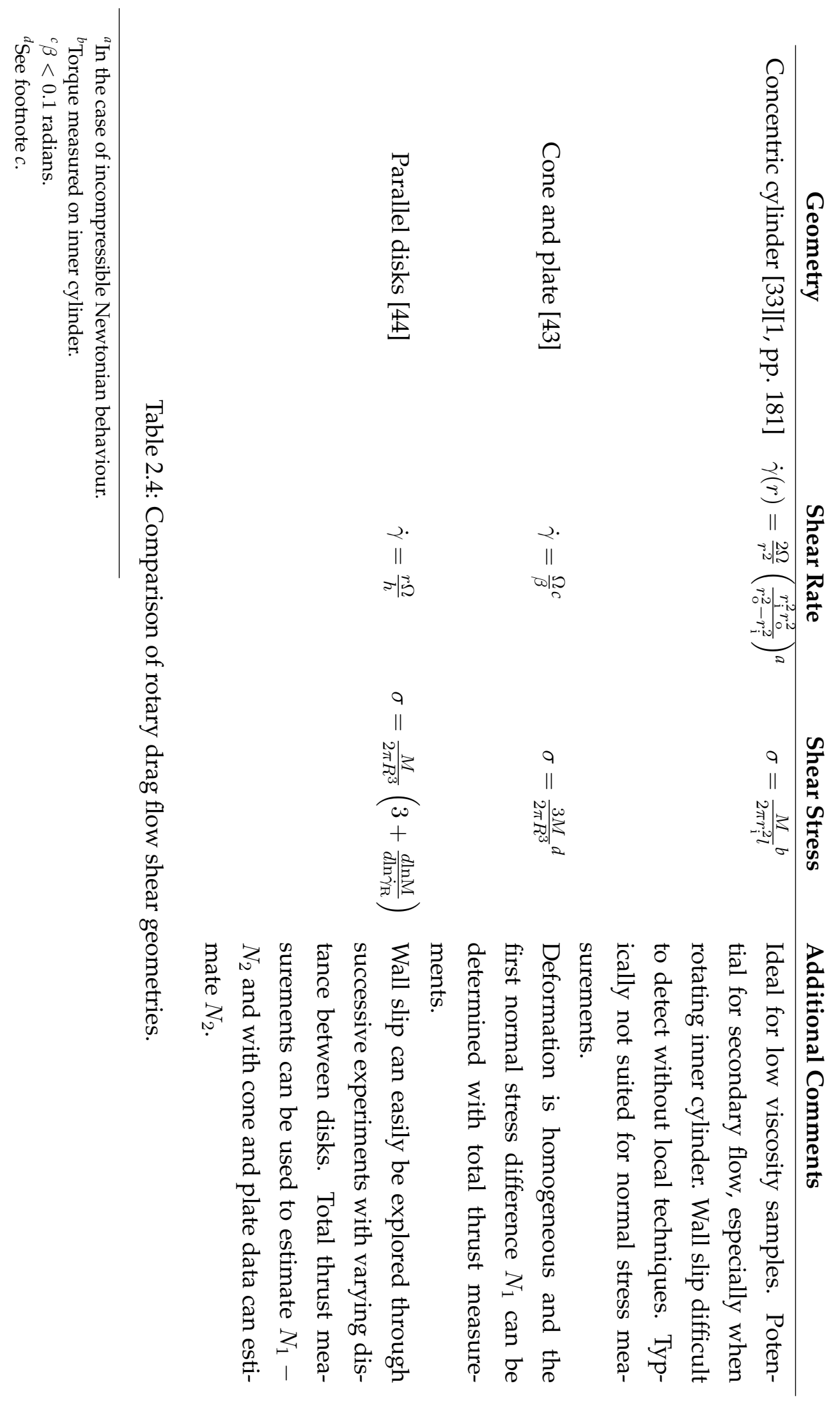


3. Time dependence

4. Extensional thickening viscosity

In the latter portion of this chapter techniques used to measure the properties of these samples were introduced with particular focus given to rotary drag flow shear methods. Two forms of rotational rheometers were introduced with a description of their evolution, the benefits of their design and the challenges of construction. Finally, three common shear geometries (concentric cylinder, cone and plate and parallel disks) were introduced, including a mathematical description of their primary characteristics (strain rate and stress) and a brief discussion of the benefits and disadvantages of each.

As the fields of rheology and rheometry continue to evolve and expand a core challenge for researchers is to perform the most appropriate test for a sample and apply the simplest model (constitutive equation) which accounts for observed physical behaviour. In this thesis new instruments have been developed to provide experimentalists with novel tools for studying simple and complex fluids within rotation drag flow shear geometries. These shear geometries are specifically designed to mirror the 'ideal' devices used in traditional rheometry, but also provide the flexibility to explore assumptions of boundary conditions and inhomogeneous deformation. Furthermore, the drive-shaft units presented in this thesis introduce new experimental methodologies which have previously been unrealised or undeveloped in the NMR community, including shear profiles (e.g. oscillatory shear) and bulk rheometry measurements (i.e. torque) acquired during NMR experiments. 


\section{Chapter 3}

\section{Nuclear Magnetic Resonance}

\subsection{Introduction}

The first nuclear magnet resonance measurements are attributed to the independent work lead by E.M. Purcell [45] and F. Bloch [46], an accomplishment which lead to the pair sharing the 1952 Nobel Prize in Physics "for their development of new methods for nuclear magnetic precision measurements and discoveries in connection therewith". Nuclear magnetic resonance (NMR) theory and applications have blossomed over the past 70 years. These principles are the key to aspects of modern chemical and biochemical analysis (NMR spectroscopy), diagnostic imaging in medicine (MRI) and material analysis (diffusion and solid-state NMR). This chapter introduces concepts of NMR which are crucial for understanding the application of magnetic resonance techniques. While the brief descriptions that follow may be acceptable for a practitioner of NMR methods, those interested in exploring these ideas in detail are pointed to some of the numerous textbooks available on the subject [47-51].

\subsection{Nuclear Magnetism}

As illustrated later in this chapter, the basic NMR experiment can be understood with a semi-classical vector model in which no quantum mechanics knowledge is required. However, in an effort to paint a complete picture of the intricacies and elegance of magnetic resonance techniques, a brief look into the quantum underpinnings of NMR is presented in this section. For additional information dedicated introductory texts on quantum mechanics are available [52]. 


\subsubsection{Spin}

As an entry point into the world of quantum mechanics, first consider angular momentum from the point of view of classical mechanics. In the everyday world, filled with cannonballs and planets, angular momentum can be described in two flavours. The first, orbital, is associated with motion of an object's centre of mass, while the second, spin, relates to motion about an object's centre of mass. Without being distracted by the nuance of this distinction in the classical world, moving to the quantum realm there is a fundamental difference between these forms of angular momentum. In the world of protons and electrons, particles still carry an orbital angular momentum which is "extrinsically" defined by a particle's motion in space; however, spin angular momentum $\mathbf{S}$ does not depend on any motion and is now an "intrinsic" property.

The spin of a particular species of elementary particle is quantised in increments of $1 / 2$. Electrons and quarks (the elementary particles that make up protons and neutrons) carry a spin of $1 / 2$ (and by extension the nucleons also carry a spin of $1 / 2$ ). In the case of nuclear magnetic resonance, where the net spin of an atomic nucleus is relevant, the nuclear spin is the sum of the individual nucleon spins and is represented by the symbol $I$.

From the spin angular momentum of a particle (and in the case of nuclear spin, the net spin), a magnetic dipole moment, $\mu$, can be defined as

$$
\boldsymbol{\mu}=\gamma \mathbf{S}
$$

where $\gamma$, the gyromagnetic ratio, is simply a proportionality constant. In the presence of a magnetic field, $\mathbf{B}$, the particle (or nucleus) may experience a torque resulting in parallel alignment with the external magnetic field. The energy associated with this torque can be defined by

$$
H=-\boldsymbol{\mu} \cdot \mathbf{B}
$$

and can be expanded using the relationship in Equation 3.1

$$
H=-\gamma \mathbf{S} \cdot \mathbf{B} .
$$

Considering a spin-1/2 system, such as a ${ }^{1} \mathrm{H}$ (or proton) NMR experiment, the angular momentum spin vector for each particle is defined as

$$
\mathbf{S}=(\hbar / 2) \boldsymbol{\sigma}
$$

where $\sigma$ represents the Pauli vector

$$
\boldsymbol{\sigma}=\sigma_{x} \hat{i}+\sigma_{y} \hat{j}+\sigma_{z} \hat{k}
$$


with the individual Pauli matrices defined as

$$
\sigma_{x} \equiv\left(\begin{array}{cc}
0 & 1 \\
1 & 0
\end{array}\right), \quad \sigma_{y} \equiv\left(\begin{array}{cc}
0 & -i \\
i & 0
\end{array}\right), \quad \sigma_{z} \equiv\left(\begin{array}{cc}
1 & 0 \\
0 & -1
\end{array}\right) .
$$

If this system is placed within a uniform magnetic field, in this example applied in the $\mathrm{z}$-direction $\left(\mathbf{B}=B_{o} \hat{\mathrm{k}}\right)$, the Hamiltonian associated with this scenario takes the form

$$
\mathbf{H}=-\gamma B_{o} \mathbf{S}_{z}
$$

or, alternatively, in matrix form

$$
\mathbf{H}=-\frac{\gamma B_{o} \hbar}{2}\left(\begin{array}{cc}
1 & 0 \\
0 & -1
\end{array}\right)
$$

where the eigenstates of $H$ indicate two distinct energy levels, $\pm \frac{\gamma B_{o} \hbar}{2}$, as depicted in Figure 3.1, with a net energy difference of $\gamma B_{o} \hbar$. This splitting is known as the Zeeman interaction where, generally, $2 I+1$ orientations (or energy levels) are available.

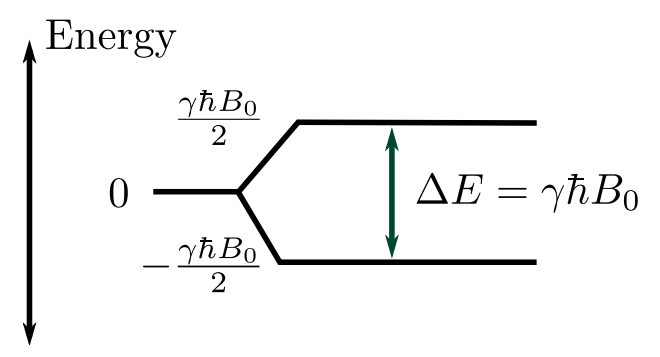

Figure 3.1: Depiction of the energy levels for a spin-1/2 system due to the Zeeman interaction.

For the ensemble in the previous example, there will be a significant portion of spins in the low energy state ("spin up", $E_{\uparrow}$, aligned parallel with the magnetic field) $-\frac{\gamma B_{0} \hbar}{2}$; additionally there will be a large number of spins in the high energy state ("spin down", $E_{\downarrow}$, aligned anti-parallel) $\frac{\gamma B_{o} \hbar}{2}$. This leads to polarisation of the population which is defined by the Boltzmann distribution

$$
\frac{N_{\downarrow}}{N_{\uparrow}}=e^{\left(-\frac{\gamma \hbar B_{o}}{k_{B} T}\right)}
$$

where $k_{B}$ is the Boltzmann constant and $T$ the absolute temperature.

As a consequence of the Boltzmann distribution, a bulk magnetisation, $M_{o}$ can be given by ${ }^{1}$

$$
M_{o}=\frac{N \gamma^{2} \hbar^{2} B_{o}}{4 k_{b} T}
$$

\footnotetext{
${ }^{1}$ Worked derivation available [53, chp. 1$]$.
} 
where $N$ is the total number of spins $N=N_{\uparrow}+N_{\downarrow}$. This net magnetisation will be a starting point for discussing the basics of an NMR experiment using a semi-classical model in Section 3.3

\subsubsection{Precession}

The two eigenstates $(\chi)$ of $H$ in Equation 3.8 can be used in the time-dependent Schrödinger equation

$$
i \hbar \frac{\partial \chi(t)}{\partial t}=H \chi(t)
$$

to express a general solution

$$
\chi(t)=\left(\begin{array}{c}
\cos (\alpha / 2) e^{i \gamma B_{o} t / 2} \\
\sin (\alpha / 2) e^{-i \gamma B_{o} t / 2}
\end{array}\right)
$$

where the expectation value for the spin vector is generally defined as

$$
\langle\boldsymbol{S}\rangle=\chi(t)^{\dagger} \boldsymbol{S} \chi(t)
$$

Using the definition in Equation 3.4 the expectation value of the spin vector can be found

$$
\langle\boldsymbol{S}\rangle=\left(\begin{array}{c}
\frac{\hbar}{2} \sin \alpha \cos \left(\gamma B_{o} t\right) \\
-\frac{\hbar}{2} \sin \alpha \sin \left(\gamma B_{o} t\right) \\
\frac{\hbar}{2} \cos \alpha
\end{array}\right)
$$

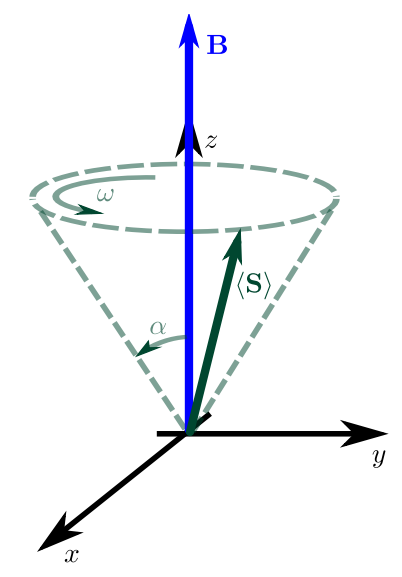

Figure 3.2: The expectation value for the spin vector $(\langle\boldsymbol{S}\rangle)$ in an external magnetic field $(\boldsymbol{B})$ precesses around the field at the Larmor frequency $(\omega)$ at a constant angle $(\alpha)$. Note this figure is not drawn to scale.

As depicted in Figure 3.2, the solution in 3.14 indicates that the spin vector is tilted from the $z$-axis at a constant angle of $\alpha$ and precesses around the magnetic field at an angular frequency

$$
\omega_{o}=\gamma B_{o}
$$


which is defined as the Larmor frequency. The Larmor frequency is determined by both the strength of the external magnetic field and the gyromagetic ratio for the particle (or in the case of NMR, the nuclei) under investigation. The experiments in this thesis have been conducted at three field strengths for which the resonance frequency of each is listed in Table 3.1.

\begin{tabular}{ccc}
$\boldsymbol{B}_{\boldsymbol{o}} / \mathbf{T}$ & $\boldsymbol{\omega}\left({ }^{\mathbf{1}} \mathbf{H}\right) / \mathbf{M H z}$ & $\boldsymbol{\omega}\left({ }^{\mathbf{2}} \mathbf{H}\right) / \mathbf{M H z}$ \\
\hline 5.9 & 250 & 38.6 \\
7.0 & 300 & 45.8 \\
9.4 & 400 & 61.4
\end{tabular}

Table 3.1: Larmor frequency as a function of magnetic field strength for proton and deuterium NMR

\subsection{Fundamentals of an NMR Experiment}

Based on the quantum mechanical descriptions in Section 3.2 the spin of a particle placed in an external magnetic field results in discrete quantised energy levels and precession at the Larmor frequency around the applied field. In terms of a macroscopic sample, the number of spins is on the order of the Avogadro constant $\left(N \approx 10^{23}\right)$ and thus an ensemble average is treated in lieu of individual spins. This concept is depicted in Figure 3.3 where the sum of all individual spin vectors is called the macroscopic magnetisation $M_{o}$.

Armed with a macroscopic quantity, the net magnetisation vector $\mathbf{M}$, spin dynamics can now be described through a semi-classical (or vector) model. Such a method was introduced by F. Bloch [54] where the excitation and relaxation processes of magnetisation can be described by

$$
\frac{d \mathbf{M}}{d t}=\gamma \mathbf{M} \times \mathbf{B}-\mathbf{R}\left(\mathbf{M}-\mathbf{M}_{o}\right)
$$

where $\mathbf{M}_{o}$ describes the steady state (thermal equilibrium) magnetisation and $\mathbf{R}$ is the relaxation matrix

$$
\mathbf{R}=\left[\begin{array}{ccc}
T_{2}^{-1} & 0 & 0 \\
0 & T_{2}^{-1} & 0 \\
0 & 0 & T_{1}^{-1}
\end{array}\right]
$$

The components of $\mathbf{R}, T_{1}$ and $T_{2}$, describe the relaxation processes in the longitudinal $(z)$ and transverse $x y$ directions, respectively. Methods for measuring these processes will be discussed in Section 3.5. 


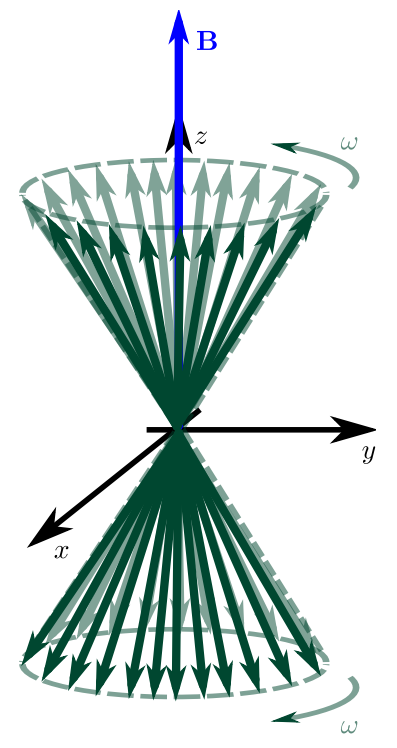

(a)

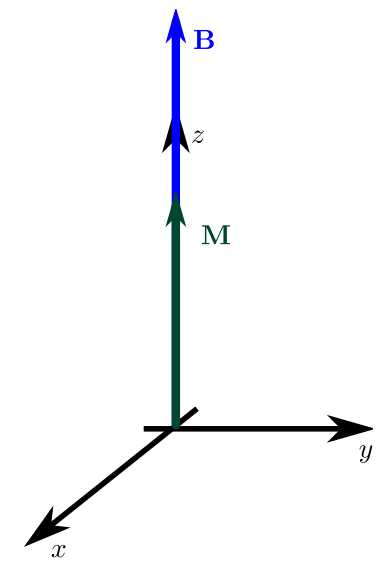

(b)

Figure 3.3: Depiction of individual spin vectors aligned parallel and anti-parallel with applied magnetic field B (a) and the net sum macroscopic magnetisation vector $\mathbf{M}$.

For an arbitrary magnetic field $\mathbf{B}(t)=\left(B_{x}(t) \hat{\mathbf{i}}, B_{y}(t) \hat{\mathbf{j}}, B_{z}(t) \hat{\mathbf{k}}\right)$ and magnetisation vector $\mathbf{M}(t)=\left(M_{x}(t) \hat{\mathbf{i}}, M_{y}(t) \hat{\mathbf{j}}, M_{z}(t) \hat{\mathbf{k}}\right)$, the three components of the Bloch equations become

$$
\begin{aligned}
& \frac{d M_{x}(t)}{d t}=\gamma\left[M_{y}(t) B_{z}(t)-M_{z}(t) B_{y}(t)\right]-\frac{M_{x}(t)}{T_{2}} \\
& \frac{d M_{y}(t)}{d t}=\gamma\left[M_{z}(t) B_{x}(t)-M_{x}(t) B_{z}(t)\right]-\frac{M_{y}(t)}{T_{2}} \\
& \frac{d M_{z}(t)}{d t}=\gamma\left[M_{x}(t) B_{y}(t)-M_{y}(t) B_{x}(t)\right]-\frac{M_{z}(t)-M_{o}}{T_{1}}
\end{aligned}
$$

where in this thesis it will been assumed that at thermal equilibrium the magnetisation is aligned with the $z$ axis, i.e. $\mathbf{M}_{o}=\left(M_{o} \hat{\mathbf{k}}\right)$. The transverse $x$ and $y$ components can be simplified by defining the complex quantities $M_{x y}(t)=M_{x}(t)+i M_{y}(t)$ (referred to as the transverse magnetisation) and $B_{x y}(t)=B_{x}(t)+i B_{y}(t)$. Thus the two transverse Bloch equations can be replaced with

$$
\frac{d M_{x y}(t)}{d t}=-i \gamma\left[M_{x y}(t) B_{z}(t)-M_{z}(t) B_{x y}(t)\right]-\frac{M_{x y}(t)}{T_{2}} .
$$

Temporarily neglecting the influence of relaxation, in the case of a static magnetic field $\mathbf{B}(t)=\left(B_{o} \hat{\mathbf{k}}\right)$, the change in magnetisation with respect to time for an arbitrarily 
orientated net magnetisation $\mathbf{M}(t)=\left(M_{x}(t) \hat{\mathbf{i}}, M_{y}(t) \hat{\mathbf{j}}, M_{z}(t) \hat{\mathbf{k}}\right)$ becomes

$$
\begin{aligned}
\frac{d M_{x y}(t)}{d t} & =-i \gamma M_{x y}(t) B_{o} \\
\frac{d M_{z}(t)}{d t} & =0 .
\end{aligned}
$$

Solving the differential equation for the transverse magnetisation and the (trivial) case of the longitudinal magnetisation $M_{z}$

$$
\begin{aligned}
M_{x y}(t) & =M_{x y}(0) e^{-i \gamma B_{o} t} \\
M_{z}(t) & =M_{z}(0)
\end{aligned}
$$

Indicating that the net magnetisation vector precesses about the longitudinal axis at the Larmor frequency $\omega_{o}=\gamma B_{o}$. A result similar to that found in the previous section.

\subsubsection{Excitation, Detection and Relaxation}

The three core components of all NMR experiments are excitation of the magnetisation, signal detection and relaxation effects. These concepts will be explored in this section.

Thermal equilibrium of the net magnetisation, in which the net magnetisation vector is aligned with the longitudinal axis, can be perturbed (excited) by applying a second magnetic field typically referred to as the $\mathbf{B}_{1}$ field. Practically, this oscillating magnetic field can be described by $\mathbf{B}_{1}=\left(B_{1} \cos \left(\omega_{r f} t+\phi\right) \hat{\mathbf{i}},-B_{1} \sin \left(\omega_{r f} t+\phi\right) \hat{\mathbf{j}}\right)$. The Bloch equations can be used to visualise the evolution of the magnetisation due to the $\mathbf{B}_{1}$, however, it is convenient to first consider an alternative coordinate system. In the examples above the magnetisation was observed from a stationary point in the laboratory reference frame. Consider a new reference frame which is rotating about the longitudinal axis $z$; this appropriately called rotating frame is observed from the perspective of the oscillating $\mathbf{B}_{1}$ field. The following transformations can be used to move between the two where $(X, Y, Z)$ terms denote the rotating frame and $(x, y, z)$ is reserved for the laboratory frame

$$
\begin{aligned}
M_{X Y}(t) & =e^{i \omega_{r f} t} M_{x y}(t) \\
M_{Z}(t) & =M_{z}(t) .
\end{aligned}
$$

When the frequency of the $\mathbf{B}_{1}$ field matches the Larmor frequency of the system under investigation $\omega_{r f}=\gamma B_{o}=\omega_{o}^{2}$, the utility of the coordinate transformation can be illustrated using the results found in Equations 3.21a and 3.21b. In the rotating frame the Bloch equations are used to describe an arbitrary magnetisation vector in a static

\footnotetext{
${ }^{2}$ Typical frequencies of the $\mathbf{B}_{1}$ field are in the radiofrequency range and thus the notation $\omega_{r f}$ is used.
} 
magnetic field

$$
\begin{aligned}
M_{X Y}(t) & =e^{i \omega_{r f} t} M_{x y}(0) e^{-i \gamma B_{o} t}=M_{x y}(0) \\
M_{Z}(t) & =M_{z}(0)
\end{aligned}
$$

where, in principle, the magnetisation is stationary. Furthermore, as the magnetisation does not appear to precess in the vector model of the rotating frame, the static magnetic field $\mathbf{B}(t)=\left(B_{o} \hat{\mathbf{k}}\right)$ is not present (Figure 3.4a).

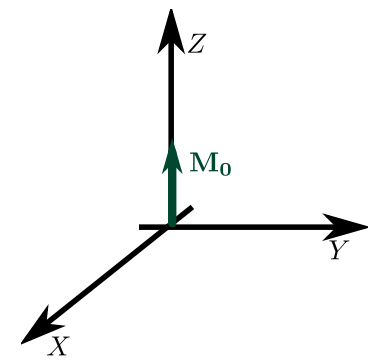

(a)

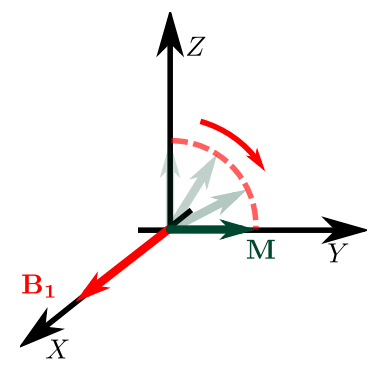

(c)

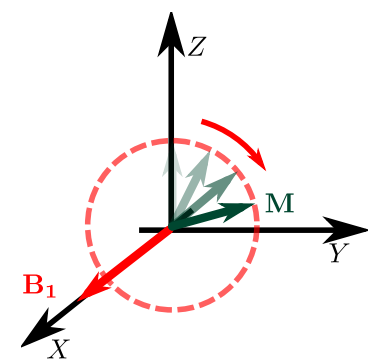

(b)

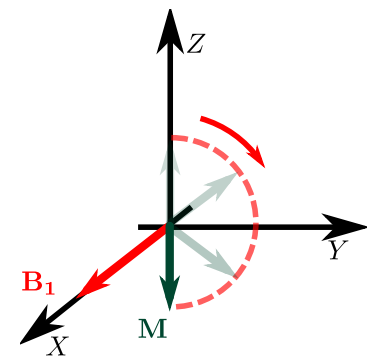

(d)

Figure 3.4: Visualising the influence of the applied magnetic field $\mathbf{B}_{1}$ in the rotating frame. At thermal equilibrium the net magnetisation is aligned with the longitudinal axis and stationary (a); the magnetisation vector begins to precess around the applied $\mathbf{B}_{1}$ field (b); by varying the duration over which the $\mathbf{B}_{1}$ field is applied the tip angle of the magnetisation vector can be controlled, e.g. $90^{\circ}$ (c) or $180^{\circ}(\mathrm{d})$.

Returning to the $\mathbf{B}_{1}$ field mentioned earlier, using complex notation, the oscillating field can be described by $B_{x y}(t)=B_{1} \cos \left(\omega_{r f} t+\phi\right)-i B_{1} \sin \left(\omega_{r f} t+\phi\right)$. When transformed to the rotating frame using the method described in Equation 3.22a this becomes $B_{X Y}=B_{1} e^{-i \phi}$. So in the rotating frame the $\mathbf{B}_{1}$ field is static and oriented in a direction controlled by the phase shift $\phi$.

The application of $\mathbf{B}_{1}$ in the rotating frame results in the precession of the magnetisation vector with the axis of rotation aligned in the direction of the applied field (Figure 3.4b). By controlling the amount of time $t_{p}$ for which the $\mathbf{B}_{1}$ field is applied the 
exact rotation of the magnetisation vector $\theta$ can be controlled

$$
\theta=\gamma B_{1} t_{p}
$$

where $\theta$ is frequently referred to as the tip, flip or nutation angle. As $t_{p}$ is typically short $\left(10^{-5}-10^{-3} \mathrm{~s}\right)$ and $\omega_{r f}$ is typically in the radiofrequency range, the $\mathbf{B}_{1}$ is frequently referred to as a radiofrequency $(\mathrm{RF})$ pulse.

In the rotating frame once the net magnetisation vector has been rotated away from the longitudinal axis the transverse components of individual spins begin to precess at different frequencies $\Omega_{i}=\omega_{o, i}-\omega_{r f}$, where $\omega_{o, i}$ is the Larmor frequency for the $i$ th spin. Following Faraday's Law of Induction, the Fourier sum of the precession of all components within the transverse plane can be detected through an induced voltage in the resonator coil as seen in Figure 3.5 and is referred to as the NMR signal. Practically, the coil which measures the precession was previously used to generate the excitation RF pulse which started the experiment.

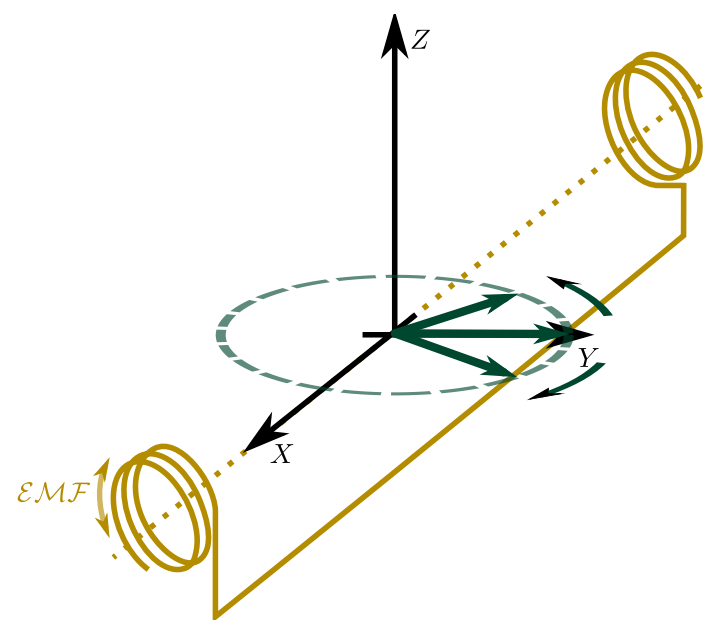

Figure 3.5: The precession of the net magnetisation $\mathbf{M}$ induces an $\mathcal{E M F}$ in the resonant coil.

Consider now the net magnetisation some time after the application of an RF pulse, remembering that in the rotating frame the magnetic field $\mathbf{B}=0$

$$
\begin{aligned}
\frac{d M_{X Y}(t)}{d t} & =-\frac{M_{X Y}}{T_{2}} \\
\frac{d M_{Z}(t)}{d t} & =-\frac{M_{Z}-M_{o}}{T_{1}} .
\end{aligned}
$$

The solutions of which are

$$
\begin{aligned}
M_{X Y}(t) & =M_{X Y}(0) e^{-t / T_{2}} \\
M_{Z}(t) & =M_{Z}(0) e^{-t / T_{1}}+M_{o}\left(1-e^{-t / T_{1}}\right)
\end{aligned}
$$


where the $M_{X Y}(0)$ and $M_{Z}(0)$ are the magnetisation components directly after the application of an RF pulse.

The relaxation mechanism in the transverse plane is the spin-spin relaxation with the characteristic time $T_{2}$. As the net magnetisation precessing within the transverse plane is the vector sum of numerous $\left(10^{23}\right)$ individual spins the spin-spin relaxation describes the loss of coherence between these spins. Along the longitudinal axis the relaxation mechanism is the spin-lattice relaxation which describes how spins reach thermal equilibrium and is characterised by the time constant $T_{1}$. Methods of determining both $T_{1}$ and $T_{2}$ will be discussed in Section 3.5.

\subsubsection{Free Induction Decay}

At this point, a simple NMR experiment can be designed where the measurement of the free induction decay (FID) results from a single pulse. The pulse programme schematic for this experiment is shown in Figure 3.6. To obtain the maximum possible signal a pulse is applied to rotate the net magnetisation by $90^{\circ}$. As previously described, the precession of the transverse components of the magnetisation induces a sinusoidal signal with exponential decay in the resonator.

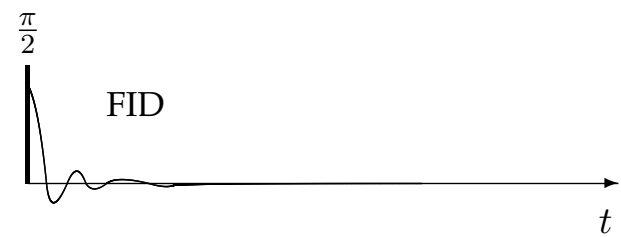

Figure 3.6: NMR pulse sequence for single pulse and signal acquisition.

The pulse sequence shown in Figure 3.6 shows the relative timing of an experiment. Short RF pulses are depicted by vertical bars where the width indicates $t_{p}$. As this chapter progresses additional building blocks will be introduced in more sophisticated pulse sequences. To denote the time at which measurements are made it is common to depict the form of the expected NMR signal. In the case of the single pulse experiment a depiction of the FID is shown.

Prior to visualising the measured NMR signal a brief discussion regarding modern NMR spectrometers is necessary. Before the instrument can store the analogue signal generated in the resonator coil the signal must be converted to digital. This process is achieved with an analogue-to-digital converter (ADC), however, the oscillation of this signal $\left(\approx \omega_{o}\right)$ may or may not be too fast for the ADC. As such the NMR signal is mixed with a reference frequency set to $\omega_{r f}$ to generate a signal at the relative frequency 
$\omega_{r e l}=\omega_{o}-\omega_{r f}$. In the example of measuring an FID, using quadrature detection with respect to the reference frequency $\omega_{r f}$, the digitised signal would take the form

$$
\begin{aligned}
& S_{A}(t)=\cos \left(\omega_{\text {rel }} t\right) e^{-t / T_{2}} \\
& S_{B}(t)=\sin \left(\omega_{\text {rel }} t\right) e^{-t / T_{2}}
\end{aligned}
$$

where the two outputs can be combined into a single complex signal

$$
S(t)=S_{A}(t)+i S_{B}(t)
$$
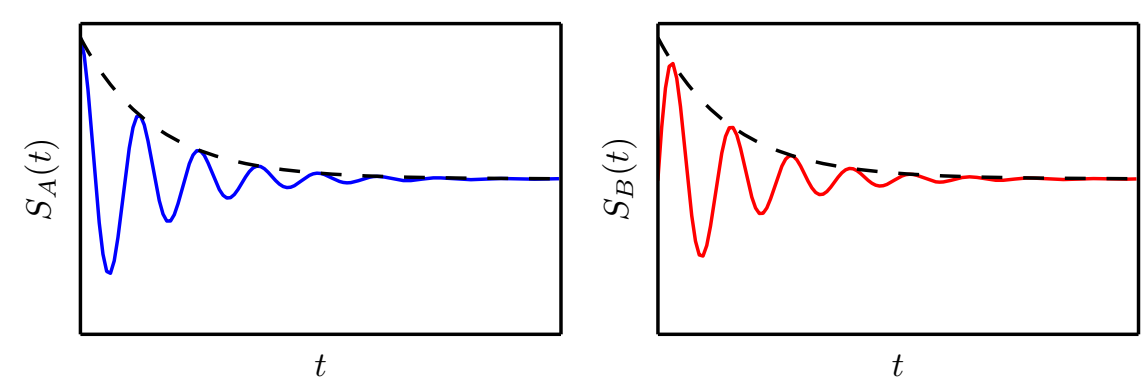

Figure 3.7: Real component (left) and imaginary component (right) of the NMR signal in a single pulse experiment.

To illustrate this result the real and imaginary components of the FID can be seen in Figure 3.7. Both parts of the signal are contained within an exponential decay envelope (depicted as dashed line in Figure 3.7). In an ideal case the time constant of this exponential decay would be $T_{2}$, however, in actual experiments this decay may be faster due to inhomogeneities in the static magnetic field which speed up the loss of coherence between spin components due to spatially varying Larmor frequencies. Thus the decay for the FID signal is characterised by $T_{2}^{*}$.

\subsubsection{Spin Echo}

Previously it was mentioned that the decay of an FID was characterised by $T_{2}^{*}$ where inhomogeneities in the static magnetic field contribute to the loss of coherence of the spin vectors as each individual spin packet has its own local Larmor frequency. Provided spins do not move, and thus always experience the same frequency of precession, it is possible to cancel the effects of $T_{2}^{*}$ through the use of a spin echo [55].

A pulse sequence depicting a spin echo can be seen in Figure 3.8. At the beginning of the experiment, a $90^{\circ} \mathrm{RF}$ pulse oriented along the $X$ axis is applied for $t_{p}$ to rotate the net magnetisation vector into the transverse plane (Figure 3.9a). Following the excitation, in the rotating frame, the individual spin components begin to precess at the 


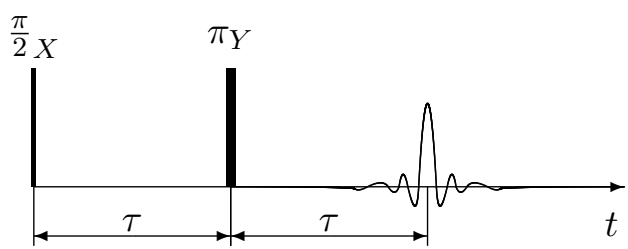

Figure 3.8: Spin echo pulse sequence.

frequency defined by the difference between their individual local Larmor frequency and the frequency of the RF pulse $\omega_{o, i}-\omega_{r f}$, causing the net magnetisation to lose coherence (Figures 3.9b and 3.9c). At a time $\tau$ after the first pulse was applied a second pulse is activated for $2 \times t_{p}$ in the direction of the $Y$ axis. By doubling the pulse time the tip angle becomes $180^{\circ}$ and the orientation of the pulse results in a rotation around the $Y$ axis. As seen in Figure 3.9d this flips the transverse plane by $180^{\circ}$ around the $Y$ axis. Afterwards, the individual spins continue to precess, but due to the inversion of the transverse plane, the direction of precession has changed (Figure 3.9e) such that at a time $\tau$ after the second RF pulse the net magnetisation is refocused (Figure 3.9f). While the spin echo is extremely practical for reducing the effects of $T_{2}^{*}$ it can not be used to negate relaxation due to $T_{2}$. The utility of the spin echo will be demonstrated as more sophisticated pulse sequences are presented in the remainder of this thesis.

\subsubsection{Signal Averaging}

Given the population distributions (Equation 3.9) for proton NMR at room temperature, typical laboratory magnetic field strengths and the resulting magnitude of the magnetisation vector (Equation 3.10), it is astonishing that the precession of the net magnetisation can induce a measurable voltage. To further increase the NMR signal above background noise it is typical to add together successive transient scans. Due to the reproducibility of the NMR signal it adds coherently while the background noise adds randomly. By repeating an experiment $N$ times the desired signal improves as a factor of $N$. Simultaneously, the root mean square of the noise is constant but uncorrelated which causes the noise to grow proportional to $\sqrt{N}$. Comparing these two terms

$$
\frac{N}{\sqrt{N}}=\sqrt{N}
$$

Thus the signal-to-noise ratio improves at a factor of $\sqrt{N}$. For an in depth treatment of the signal-to-noise ratio see the manuscript from D.I. Hoult and R.E. Richards [56]. 


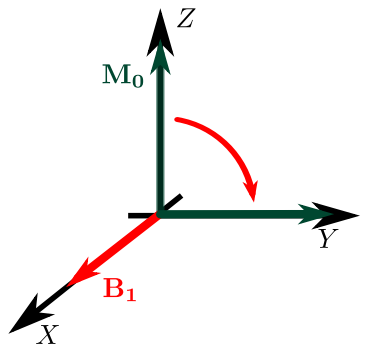

(a)

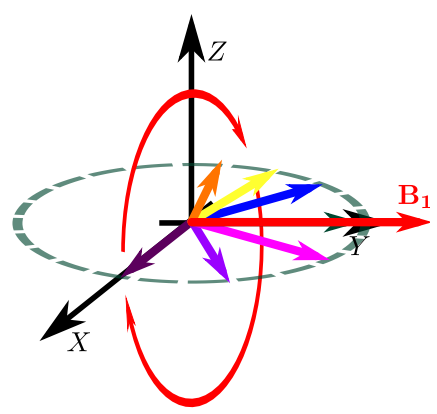

(d)

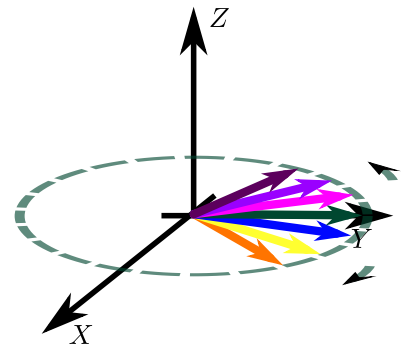

(b)

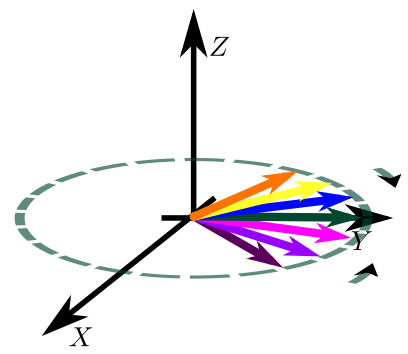

(e)

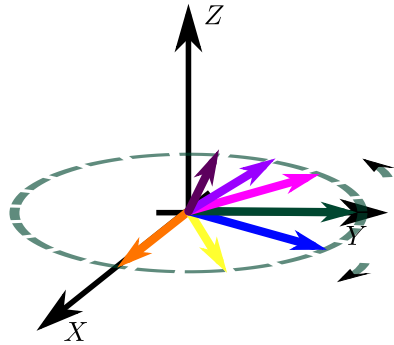

(c)

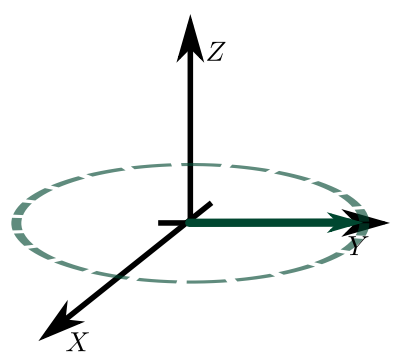

(f)

Figure 3.9: Influence of the spin echo on spin evolution.

\subsubsection{Phase Cycling}

Thinking back to the single pulse experiment used to measure the FID, consider the direction in which the $90^{\circ} \mathrm{RF}$ pulse was applied. In principle the same NMR signal would be measured regardless of the orientation of the $\mathbf{B}_{1}$ field although practically an extra post-processing step would be required to orient the phase of the signal. If two successive single pulse experiments were performed where the excitation pulses were oriented in opposite directions (e.g. $X$ and $-X$ ), due to the reproducibility of the NMR signal, the results of these experiments should be the same except for a phase difference. Thus cycling the orientation (phase) of RF pulses provides a method to identifiy systematic errors such as imperfect pulse timing and baseline artefacts. Furthermore, through the appropriate addition or subtraction of successive scans the effects of these experimental errors can be reduced.

Additionally, appropriate phase cycling can be used to both retain desired parts of the NMR signal or eliminate contributions that have no significance. Phase of both RF pulses and the receiver can be varied and the sophistication of these schemes increases with the complexity of the NMR experiment. Methods to determine the appropriate 
phase cycle scheme for a specific pulse programme include the coherence-transfer pathway approach [57].

\subsection{NMR Spectroscopy}

The NMR signal can be used to characterise a sample's chemical spectra.

\subsubsection{Proton Spectroscopy}

In the case of a static and homogeneous magnetic field the local molecular environment surrounding an individual spin can alter the local magnetic field and thus change the Larmor frequency of an individual spin. Previously it was mentioned that the NMR signal is a Fourier sum of all precession frequencies suggesting that the NMR signal can carry information regarding the chemical structure of a sample. To analyse such data, the NMR spectrum, displaying signal intensity as a function of frequency is generated from a Fourier transformation of the measured time domain data

$$
\begin{gathered}
F\{S(t)\}=s(f)=\int_{-\infty}^{\infty} S(t) e^{(i 2 \pi f t)} d t \\
F^{-1}\{s(f)\}=S(t)=\int_{-\infty}^{\infty} s(f) e^{(-i 2 \pi f t)} d f
\end{gathered}
$$

where $F$ and $F^{-1}$ represent the Fourier transform (FT) and inverse Fourier transform (IFT), respectively. In Figure 3.10 the real and imaginary components of FID signal are shown in both the time and frequency domains.
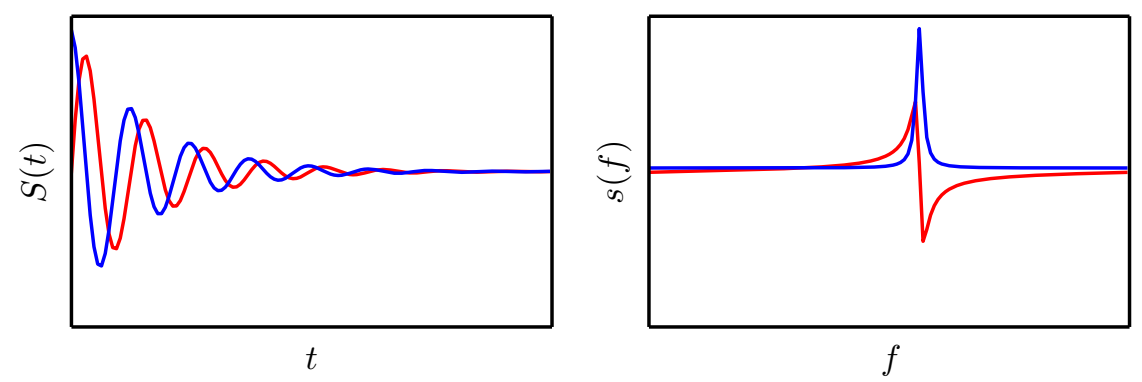

Figure 3.10: Visualising the real (blue) and imaginary (red) components of the FID signal in both the time domain (left) and frequency domain (right).

Mechanisms known to influence the local frequency of precession, and thus allow the NMR spectrum to describe the chemical environment, include: chemical shift, due to electron shielding of the magnetic field [58] and scalar coupling, due to the interaction of neighbouring nuclei [51, sec. 4.4.3]. 


\subsubsection{Deuterium Spectroscopy}

So far all discussion has focused on spin- $1 / 2$ systems. While in principle the methods described in this chapter can be applied to numerous isotopes with nuclear spin $I=1 / 2$ (e.g. ${ }^{1} \mathrm{H},{ }^{13} \mathrm{C}$ and ${ }^{19} \mathrm{~F}$ ), due to both isotopic natural abundance and the prevalence of water (thus hydrogen atoms) in nature, the majority of liquid state experiments focus on the ${ }^{1} \mathrm{H}$ nuclei. However, there are advantages to NMR techniques of systems with $I>1 / 2$. Nuclei with spin $I>1 / 2$ exhibit a quadrupolar moment which interacts with the electric field gradient generated by surrounding electrons and comprise over $65 \%$ of NMR active nuclei. In general, quadrupolar interactions are of great value to the field of solid state NMR. Pertaining to soft matter one specific nuclei, deuterium $\left({ }^{2} \mathrm{H}\right.$; $I=1$ ), is sensitive to molecular alignments due to quadrupolar interactions and can be used to derive order parameters from an NMR spectrum [59].

\subsection{Relaxometry}

In earlier discussions the relaxation time constants $T_{1}$ and $T_{2}$ were introduced. In this section basic methods for measuring these relaxation parameters will be discussed.

\subsubsection{Spin-Lattice Relaxation}

Experimentally, knowledge of $T_{1}$ is crucial when running successive transient scans (e.g. signal averaging). For any NMR experiment if there is signal available to measure the magnetisation the sample is not at thermal equilibrium. Thus between the end of a scan and the beginning of the next experiment a time delay is necessary to allow the magnetisation to return to equilibrium ${ }^{3}$. Common practice is to use a recovery time $\approx 5 \times T_{1}$. Furthermore, $T_{1}$ can be used to describe material properties [61] (e.g. viscosity) and may be a physical parameter of interest beyond simply determining timing for an NMR experiment.

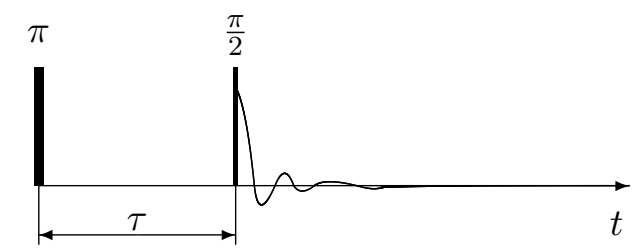

Figure 3.11: Inversion recovery pulse sequence used to determine $T_{1}$.

\footnotetext{
${ }^{3}$ There are techniques (e.g. FLASH [60]) which utilise small flip angles to retain longitudinal magnetisation and thus eliminate the delay between successive scans.
} 
One technique to measure $T_{1}$ is the inversion recovery pulse sequence [62, sec. vi] (Figure 3.11). The inversion recovery experiment begins with a $180^{\circ} \mathrm{RF}$ pulse which inverts the spin populations by flipping the orientation of the net magnetisation. Afterwards, the magnetisation returns to thermal equilibrium. At some time $\tau$, a $90^{\circ}$ pulse is applied and the resulting FID is immediately measured. Earlier in the chapter the Bloch equations were used to solve the evolution of magnetisation in the rotating frame (i.e. $\mathbf{B}=0$ ) and with no applied magnetic fields (i.e. $\mathbf{B}_{1}=0$ ); the result was given in Equation $3.26 \mathrm{~b}$. In the case of the inversion recovery sequence the magnetisation directly after the first pulse is $\mathbf{M}(0)=\left(-M_{o} \hat{\mathbf{k}}\right)$. Substituting this result into and rearranging Equation $3.26 \mathrm{~b}$ yields

$$
M(t)=M_{o}\left(1-2 e^{-t / T_{1}}\right)
$$

where the transverse components are zero based on $\mathbf{M}$ and Equation 3.26a.

By making measurements of the FID as a function of $\tau$ the first point of each FID can be plotted as a function of $\tau$. Finally, the form of Equation 3.31 can fit to the experimental data using a non-linear curve fitting routine to determine the value of $T_{1}$.

\subsubsection{Spin-Spin Relaxation}

Analogous to $T_{1}$ being used to determine repetition timing between successive NMR experiments $T_{2}$ can be used to determine the possible length of a pulse sequence. Coherence decay increases the entropy of a spin system and as the process does not involve an exchange of energy total entropy is not conserved. Therefore the process of $T_{2}$ is irreversible. Stochastic mechanisms that lead to loss of phase coherence can include molecular translation, rotational diffusion and segmental motion of molecules [51, sec 4.5]. Regardless of the cause coherence loss of the NMR signal due to $T_{2}$ cannot be recovered through any $\mathrm{RF}$ pulse excitation (e.g. spin echoes).

A common method to measure $T_{2}$ is the Carr-Purcell-Meiboom-Gill echo train [62, 63]. This pulse sequence is depicted in Figure 3.12. After a $90^{\circ}$ excitation RF pulse at time $\tau$ a $180^{\circ}$ pulse inverts the transverse plane with a spin echo forming at time $2 \tau$. After the initial spin echo the spin system begins to loss phase coherence only to be refocused with a subsequent $180^{\circ}$ pulse and produce a new spin echo. In practice the number of $180^{\circ} \mathrm{RF}$ pulses and subsequent echoes will be much greater than those illustrated in Figure 3.12 (32, 64, 128 or more echoes). The envelope of decay for the spin echo train can be defined from Equation 3.26a

$$
M(t)=M_{o} e^{-t / T_{2}}
$$

By taking a single point (echo centre) from each spin echo experimental data can be fit to Equation 3.32 and used to determine $T_{2}$. 


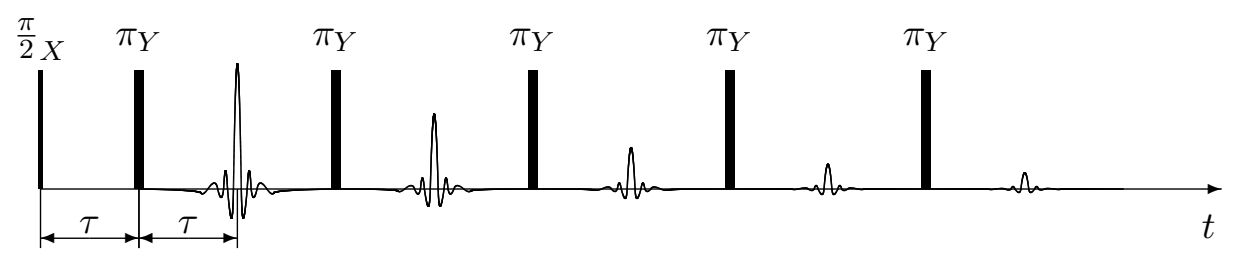

Figure 3.12: CPMG pulse sequence used to determine the $T_{2}$.

\subsection{Magnetic Field Gradients}

To this point the background field has always been idealised as static and homogeneous. Indeed these are necessary assumptions particularly in the case of high resolution NMR spectroscopy (Section 3.4.1). However, numerous techniques utilise a magnetic field that varies in time and space. The utility of which will be exploited for selective excitation (Section 3.6.1), imaging (Section 3.6.2) and encoding translational motion (Section 3.6.3).

In Equation 3.15 it was shown that the frequency of precession is determined by the product of gyromagnetic ratio and the applied magnetic field. If, in addition to the static field $\mathbf{B}=B_{o} \hat{\mathbf{k}}$, a spatial varying magnetic field $\mathbf{G}(\boldsymbol{r})$ is applied through the sample local precession will depend on position

$$
\omega(\boldsymbol{r})=\gamma\left(B_{o}+\mathbf{G}(\boldsymbol{r}) \cdot \boldsymbol{r}\right)
$$

where the $\mathbf{G}(\boldsymbol{r})$ is the gradient of the magnetic field in the $z$ direction $^{4}$

$$
\mathbf{G}(\boldsymbol{r})=\nabla B_{z}=\frac{\partial B_{z}}{\partial x} \hat{\mathbf{i}}+\frac{\partial B_{z}}{\partial y} \hat{\mathbf{j}}+\frac{\partial B_{z}}{\partial z} \hat{\mathbf{k}}
$$

In practice, NMR imaging systems have hardware (gradient sets) in which the change in $B_{z}$ along a given axis ( $x, y$ or $z$ ) can be controlled independently. To illustrate the local precession dependence on the applied gradient field consider the scenario in Figure 3.13 in which $\mathbf{G}(\boldsymbol{r})=\frac{\partial B_{z}}{\partial x} \hat{\mathbf{i}}$. In Section 3.3.2 it was mentioned that a reference frequency (typically $\omega_{r f}$ ) is subtracted from the measured signal prior to digitisation, as such the $\gamma B_{o}$ term can be ignored such that local precession is only dependent on the applied field gradient.

\subsubsection{Selective Excitation}

One use for the spatially dependent magnetic field is the ability to selectively manipulate regions of spins. Combining an RF pulse which affects only a specific region of

\footnotetext{
${ }^{4}$ The assumption has been made that the static field is much greater than the gradient field and thus concomitant fields (i.e. $\boldsymbol{r} \cdot \nabla B_{x}$ and $\boldsymbol{r} \cdot \nabla B_{y}$ ) can be ignored [51, chp. 5].
} 

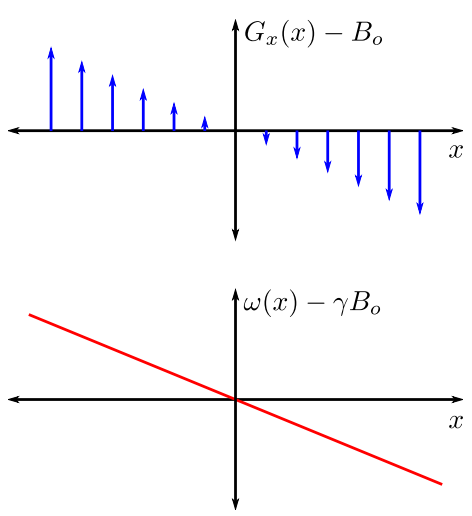

Figure 3.13: Neglecting the $B_{o}$ field, the local magnetic field varies as a function of position along the $x$ axis due to an applied gradient field $\mathbf{G}(\boldsymbol{r})=\frac{\partial B_{z}}{\partial x} \hat{\mathbf{i}}$ (top). As a result of this gradient field, the local precession (neglecting the Larmor frequency due to $\mathbf{B}$ ) shows a similar spatially dependent pattern.

precession frequencies with the gradient field discussed above, spatially selective excitation is possible. So far in discussing NMR pulse sequences, the RF pulse has been assumed to excite the entire sample within the resonator coil. In the case of short pulses $\left(t_{p} \approx 10^{-4} \mathrm{~s}\right.$ ) this assumption is reasonable, but will be motivated in this section.

The bandwidth of an RF pulse is inversely related to $t_{p}$ [47, sec. 2.4.8]. Drawing an example from Translational Dynamics $\mathcal{E}$ Magnetic Resonance [51, sec. 5.3.3], applying a tip angle $\theta=90^{\circ}$ for the duration $t_{p}=1 \mathrm{~ms}$ will selectively excite spin packets with a resonant frequency within a $1 \mathrm{kHz}$ bandwidth of the Larmor frequency. Thus, by tuning the magnetic field gradient and the RF pulse duration, spatial selective excitation is accomplished $^{5}$.

In the NMR experiments discussed earlier in this chapter, the duration of the applied RF pulses would have been sufficiently short as to excite a broadband of resonant frequencies. Typically the bandwidth of frequencies contained within these short RF pulses is sufficient such that the exact shape of the excitation spectrum is not important. However, in the case of selective (narrowband) RF pulses, the ideal form of the frequency domain spectrum would be a hat function. This excitation spectrum can be approximately achieved by applying the RF pulse as a sinc waveform. In practice, spatially selective excitation is commonly achieved through the application of a shaped RF pulses (e.g. sinc waveform) in the presence of an applied gradient field, as demonstrated in Figure 3.14.

\footnotetext{
${ }^{5}$ Similarly, a narrowband RF pulse can be used to selectively excite nuclei within a specific range of chemical shift. This method does not require magnetic field gradients.
} 


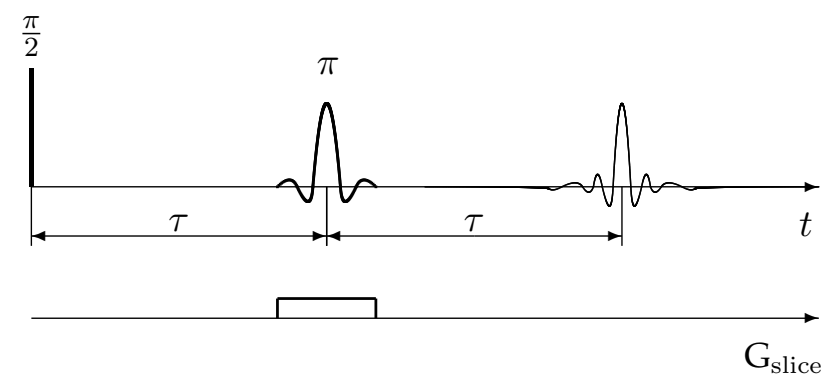

Figure 3.14: Example pulse programme for selective excitation.

\subsubsection{Magnetic Resonance Imaging}

In addition to spatial selectivity, magnetic field gradients can further be used to image the density of nuclear spins, a technique frequently referred to as magnetic resonance imaging (MRI). MRI is a common diagnostic tool in modern medicine, but similarly has applications in physical science laboratories. The first successful use of magnetic field gradients to produce a magnetic resonance image was demonstrated by P. Lauterbur in 1973 [64]; a feat which was recognised in 2003 when P. Lauterbur shared the Nobel Prize in Medicine with P. Mansfield (some of Mansfield's contributions to the development of MRI will be discussed in the following section). While numerous textbooks have been dedicated to MRI, two particular texts standout for their specific application of imaging techniques to material science $[47,65]$.

The remainder of this section is dedicated to describing how magnetic field gradients are used in MRI. As a point of reference, Figure 3.15 illustrates raw and reconstructed imaging data in the case of both a 1D and 2D experiment. The sample was a concentric cylinder geometry, which can be clearly identified in the reconstructed 2D image (lower right). The left hand column of Figure 3.15 displays the measure (k-space) data. The formalism and acquisition of which will now be discussed.

\section{k-space}

For an arbitrary sample, the nuclear spins within a small volume element $d V$ will generate a signal $d S(t)$

$$
d S(\mathbf{G}, t) \propto \rho(\mathbf{r}) d V e^{i \omega(\mathbf{r}) t}
$$

where $\rho(\mathbf{r})$ is the spin density at position $\mathbf{r}$. In the presence of magnetic field gradients, the local precession $\omega(\mathbf{r})$ is described by Equation 3.33. Ignoring any constant of proportionality, the relationship between signal and spin density can be defined as

$$
d S(\mathbf{G}, t)=\rho(\mathbf{r}) d V e^{i \gamma\left(B_{o}+\mathbf{G}(\mathbf{r}) \cdot \mathbf{r}\right) t} .
$$



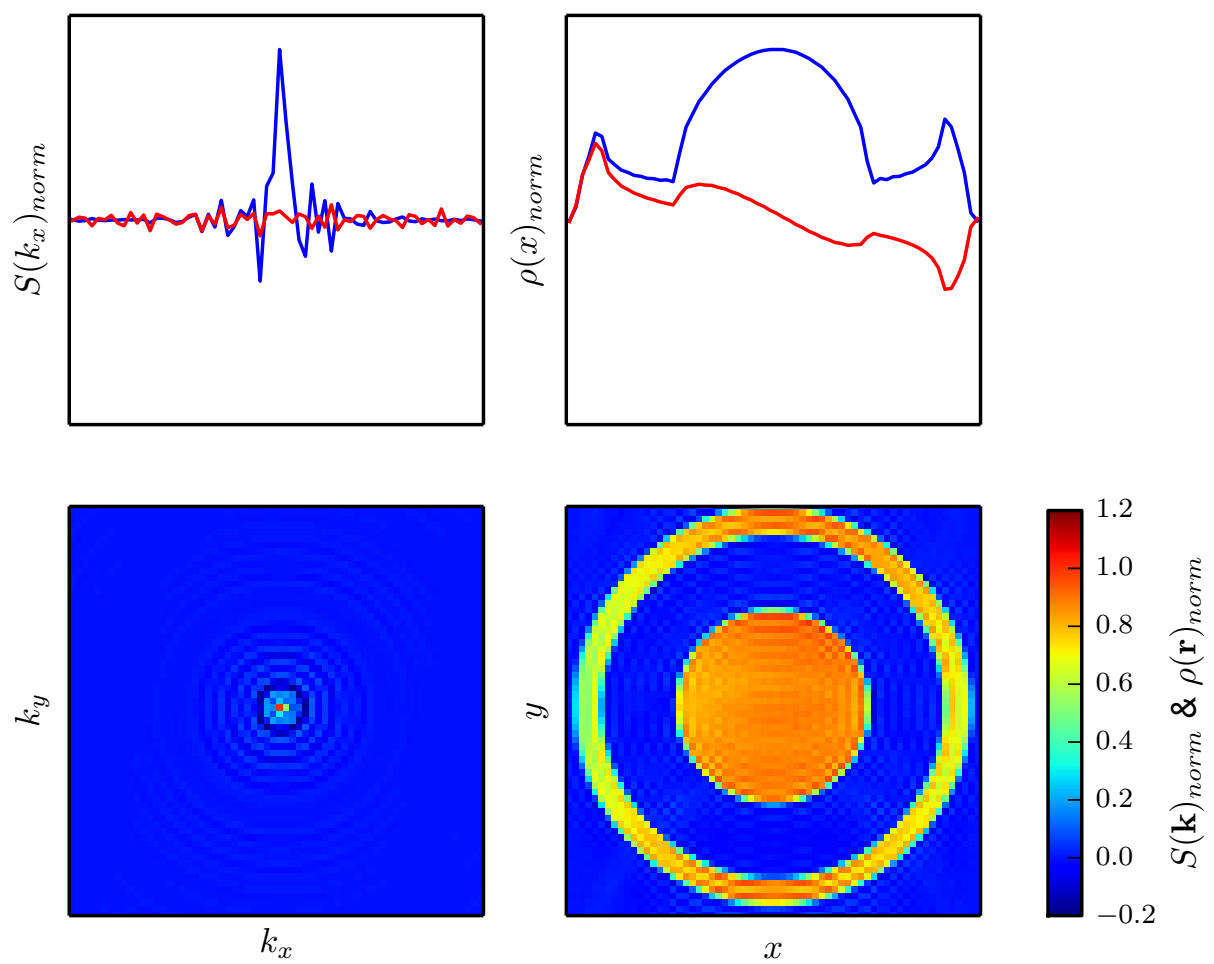

Figure 3.15: Example magnetic resonance imaging data. Raw data for a 1D imaging experiment is shown in the top left plot, where the blue trace is the real part of the NMR signal and the red trace is the imaginary component. The reconstructed $1 \mathrm{D}$ projection of proton spin densities is shown in the top right plot. The panel in the bottom left shows the real component of the $2 \mathrm{D} k$-space data while the bottom right panel contains the real part of the real space image.

Previously, it was noted that the measured NMR signal oscillates at a relative frequency $\omega_{r e l}=\omega_{o}-\omega_{r f}$. Setting the reference frequency $\omega_{r f}$ equal to $\gamma B_{o}$ causes the $\gamma B_{o}$ term to drop out of Equation 3.36 and the relationship can be described as a volume integral

$$
S(t)=\int \rho(\mathbf{r}) e^{i \gamma \mathbf{G}(\mathbf{r}) \cdot \mathbf{r}} d \mathbf{r} .
$$

In work from P. Mansfield and P.K. Grannell [66] the concept of a reciprocal space vector $\mathbf{k}^{6}$,

$$
\mathbf{k}=\frac{\gamma \mathbf{G} t}{2 \pi}
$$

\footnotetext{
${ }^{6}$ In their original work, the reciprocal space vector was defined as $\mathbf{k}=\gamma \mathbf{G} t$ with units of $\operatorname{rad~m}^{-1}$ opposed to the $\mathbf{k}$ defined in this thesis in units of $\mathrm{m}^{-1}$.
} 
was introduced and used in describing the Fourier relationship between k-space and real space

$$
\begin{aligned}
S(\mathbf{k}) & =\int \rho(\mathbf{r}) e^{i 2 \pi \mathbf{k} \cdot \mathbf{r}} d \mathbf{r} \\
\rho(\mathbf{r}) & =\int S(\mathbf{k}) e^{-i 2 \pi \mathbf{k} \cdot \mathbf{r}} d \mathbf{k} .
\end{aligned}
$$

In an MRI experiment $\mathbf{k}$-space is sampled at discrete points with reconstruction techniques used to general real space maps of spin density. In the case of sampling k-space on a Cartesian grid, the Fourier relation defines the resolution in real space $\Delta l$ as

$$
\Delta l=\frac{1}{2 k_{\max }}
$$

where $k_{\max }$ is the magnitude of the maximum $\mathbf{k}$-space vector. The field of view of the real space image is defined by

$$
F O V=\frac{N}{2 k_{\max }}
$$

where $N$ is the number of data points sampled in $\mathbf{k}$-space.

\section{k-space Acquisition}

Examination of Equation 3.38 shows that it is possible to traverse $\mathbf{k}$-space by stepping either time or gradient variables. In practice, both strategies are frequently utilised and are referred to as frequency encoding (when incrementing time) or phase encoding (in the case of varying gradient strengths). Figure 3.16 illustrates two 1D imaging pulse sequences where each uses either a frequency or phase encoding scheme. In the case

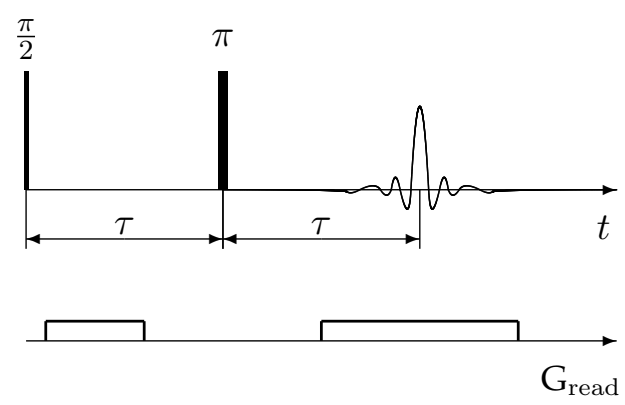

(a)

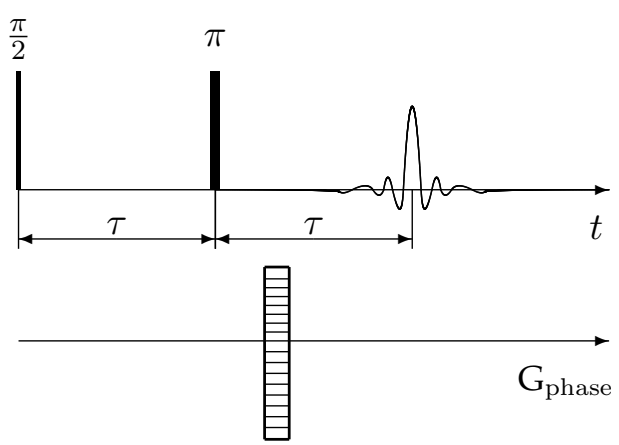

(b)

Figure 3.16: Pulse sequences for 1D imaging utilising either a frequency encoding scheme (a) or a phase encoding scheme (b).

of frequency encoding (Figure 3.16a) the NMR signal is measured in the presence of 
a magnetic field gradient (referred to as the read gradient). In acquiring the signal an entire line of $\mathbf{k}$-space is sampled and the complete 1D image (neglecting signal averaging or phase cycling) is acquired in one scan. The 1D imaging data in Figure 3.15 was acquired with frequency encoding. For phase encoding (Figure 3.16b) the imaging gradient (referred to as the phase gradient) is pulsed prior to the signal acquisition. For phase encoding, each measurement at a different gradient strength corresponds to a single point in $\mathbf{k}$-space. Using this pulse sequence successive scans are needed, each at a different gradient strength. Although the phase encoding approach would take more time to produce an equivalent 1D image to the frequency encoding method, one advantage of phase encoding is that the NMR chemical spectra has been preserved [ 51 , sec. 5.3.2].

For 2D imaging, frequency and phase encoding are commonly combined into a single pulse sequence (e.g. Figure 3.17). Utilising both of these strategies, it is possible to sample a plane of $\mathbf{k}$-space. An illustration of $2 \mathrm{D} \mathbf{k}$-space data can be seen in the bottom left panel of Figure 3.15.

As numerous rapid k-space sampling strategies exist a treatment of these pulse sequences is not feasible, but an introduction to various methods can be found in Principles of Nuclear Magnetic Resonance Microscopy from P.T. Callaghan [47, sec 3.6].

\subsubsection{NMR Velocimetry}

The final application of magnetic field gradients to be discussed involves measuring translational motion. The techniques used in this thesis for measuring fluid velocities utilise the pulsed gradient spin echo (PGSE) pulse sequence (Figure 3.18). Following the initial excitation in the PGSE experiment, ignoring any inhomogeneities in the static magnetic field, the net magnetisation precesses at the Larmor frequency $\omega_{o}=\gamma B_{o}$. When the first gradient is applied, for the duration of the pulse $\delta$, the local precession is momentarily described by Equation 3.33. Once the gradient is turned off, there exists a position dependent phase shift of the transverse magnetisation. Next, the $180^{\circ}$ pulse inverts the direction of precession and the second gradient pulse imprints an additional local phase shift. If, over the course of the experiment, the sample is stationary (e.g. no flow) and the affects of diffusion are ignored the local phase shift accumulated during the gradient pulses is negated. However, if the sample is moving in the direction of the applied gradient with a constant velocity $v$ the NMR signal will acquire a net phase shift [67]

$$
\phi=\gamma \delta g \Delta v
$$

where $g$ is the gradient strength and $\Delta$ is the time between turning on the PGSE gradients (Figure 3.18). Any inhomogeneities in the static magnetic field would cause an ad- 


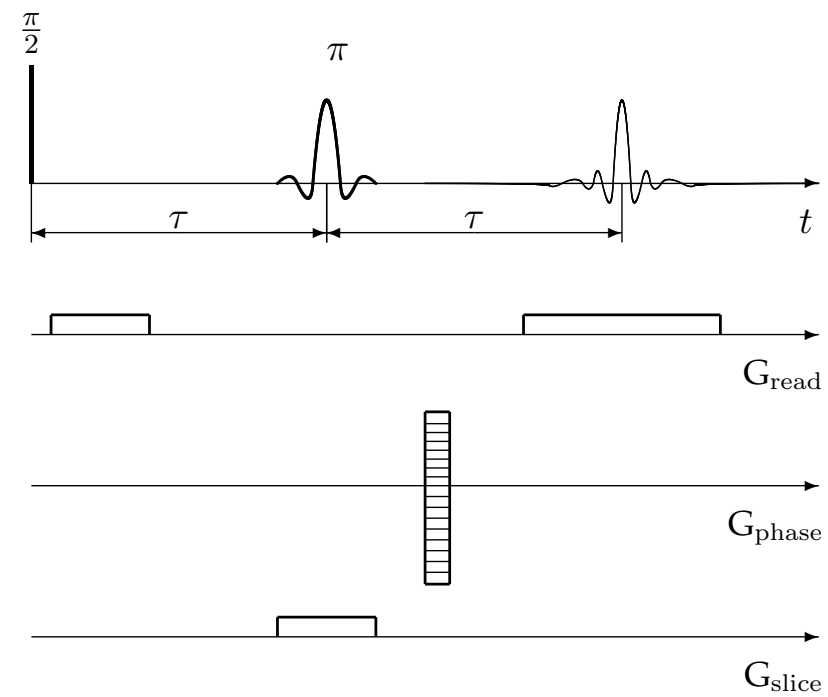

Figure 3.17: Two dimensional imaging pulse sequence which utilises both frequency and phase encoding.
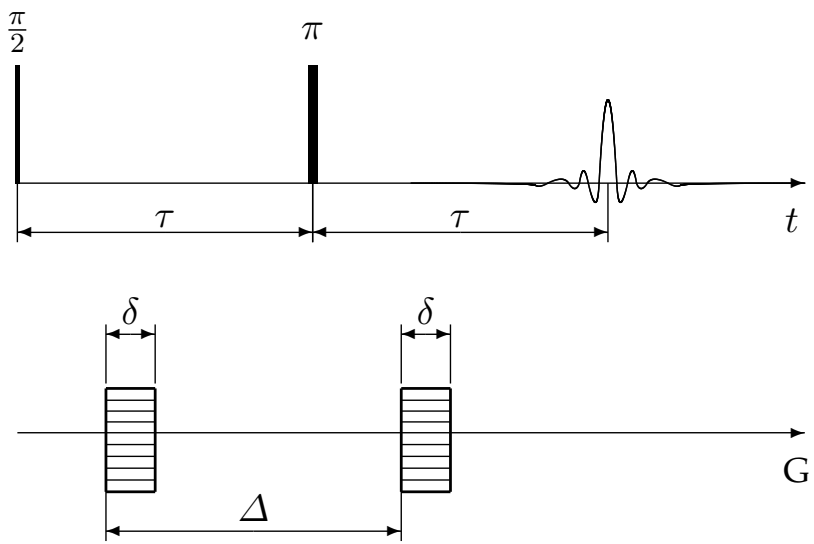

Figure 3.18: Basic pulse gradient spin echo (PGSE) pulse programme for encoding translation motion. 
ditional phase shift of the signal which would influence the determined velocity. Thus, practically, the PGSE experiment is performed at multiple gradient strengths with the velocity calculated from the relative phase shift between successive measurements. To spatially resolve fluid velocities the PGSE experiment can be combined with numerous imaging sequences.

A second technique for velocity characterisation would be to measure the average propagator [51, sec. 2.1.6][68] which carries information regarding both diffusion and flow. For this approach a new reciprocal space vector, $\mathbf{q}$, is used [51, sec. 5.6] [69]

$$
\mathbf{q}=\frac{\gamma \mathbf{G} \delta}{2 \pi} \text {. }
$$

Using q-space a Fourier relationship is formed between the NMR signal and the average propagator. For this experiment, the PGSE pulse sequence can be used to sample q-space, however, the data processing would be more complex [51, sec. 10.1.2].

\subsection{The NMR Instrument}

All experimental work presented in this thesis has been conducted in vertical bore superconducting magnets $\left({ }^{1} \mathrm{H}\right.$ resonant frequency of $\left.250-400 \mathrm{MHz}\right)$. A $400 \mathrm{MHz}$ wide bore magnet is shown in Figure 3.19. For these instruments, the polarising magnetic field is generated by a solenoid of superconducting wire in which a persistent current flows provided the coil is kept below a critical temperature.

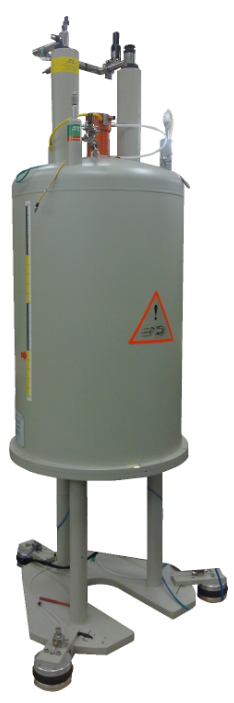

Figure 3.19: Bruker Wide Bore $400 \mathrm{MHz}$ superconducting magnet (spectrometer and operator console are not shown).

An overview of the NMR instrument can be found in Spin Dynamics from M. Levitt [48, chp. 4] with a more technical discussion available from E. Fukushima and S. Roeder [70]. 
Furthermore, principles of resonator (RF) coil design have been examined by J. Mispelter, M. Lupa and A. Briguet [71].

\subsection{Conclusion}

This chapter opened with a brief discussion of the quantum mechanic underpinnings of the nuclear magnetic resonance phenomenon, however, it was quickly explained that a semi-classical vector model would be sufficient to introduce various concepts of nuclear spin dynamics. After exploring spin excitation, signal detection and relaxation phenomenon, examples of practical spin manipulations and basic pulse sequences were illustrated. These included the single pulse (e.i. free induction decay) and spin echo experiments in addition to methods for characterising NMR relaxation times, such as the inversion recovery experiment or use of the Carr-Purcell-Meiboom-Gill echo train to measure $T_{1}$ and $T_{2}$ respectively. Finally, magnetic field gradients were shown to be useful for spatially selective excitation, magnetic resonance imaging (MRI) and encoding translation motion (e.g. diffusion and flow). The various concepts presented in this chapter are the basic ingredients for modern NMR experiments and will be frequently used through this thesis. 


\section{Chapter 4}

\section{Previous NMR Studies of Materials Under Shear}

\subsection{Introduction}

One of the earliest uses of the term Rheo-NMR is found in a paper from A.I. Nakatani and colleagues [72] published in 1990 where a cone and plate geometry was used with ${ }^{1} \mathrm{H}$ NMR spectroscopy to study the response of polymer fluids to shear-induced anisotropy. Later, in 1993, the term was used by L.N. Goncalves and colleagues [73] to describe a technique previously developed by the authors in 1986 [74]. This early manuscript looked at ordering parameters of polymers aligned with the magnetic field of an NMR spectrometer and to characterise the viscosity; the sample tube was rotated relative to the magnetic field and the recovery of the director field to equilibrium was measured via spectroscopy. Although this work predates that of the Nakatani group by four years it did not involve a shear geometry which dominates current Rheo-NMR studies. Today studies frequently utilise small continuous drag flow shear devices [75] (e.g. concentric cylinder, cone and plate) or pressure driven pipe flow to study the rheology of materials with NMR techniques [76-80].

In the modern literature the use of the term Rheo-NMR seems to be subjective; in some cases a historical perspective (as described above) seems to influence some authors, while at times Rheo-NMR is a nod to the commercially available hardware kit (described in Section 4.3). Furthermore, perhaps in an attempt to differentiate emerging techniques, new terms have evolved such as MRI-rheology [81] and in the medical community, flow-MRI [82]. As a result, it is difficult to both definitively identify the origin of the term Rheo-NMR and define a specific meaning for its use. Regardless, there is clear evidence that the application of nuclear magnetic resonance techniques to investigate rheological properties of complex materials and materials under shear 
extends back at least three decades.

The work presented in this thesis is self branded as a new approach to studying materials under shear with nuclear magnetic resonance. As such, it is necessary to not only understand the context of the hardware and experiment but also the history of previous experimental set-ups. A few substantial reviews of Rheo-NMR techniques have been published by a notable pioneer in the field [83, 84], however, in the time since the manuscripts were published there have been significant developments in the magnetic resonance microscopy community that have impacted Rheo-NMR. Additionally, included in this chapter is a brief introduction to magnetic resonance techniques that previously have not been branded as Rheo-NMR but can be used to investigate properties relevant to a soft matter scientist.

\subsection{Motion Encoding Pulse Sequences and NMR Signal}

Velocimetry is one NMR technique used to study materials under shear; as a spatially resolved measurement, velocity images provide insight into local phenomenon such as shear banding. Over the past decade, significant progress has been made in developing pulse sequences for the rapid acquisition of velocity data with the potential to observe turbulent phenomenon in flowing materials. A review of fast imaging methods can be found in Translational Dynamics \& Magnetic Resonance by P.T. Callaghan [51, sec. 10.3].

Of the pulse sequences discussed in the reference above, PGSE encoding coupled with rapid acquisition relaxation enhanced (RARE) imaging balances both temporal resolution and experimental robustness. Although it is not the fastest velocity imaging sequence ${ }^{1}$ PGSE-RARE can be used to acquire $2 \mathrm{D}$ velocity maps in approximately $2 \mathrm{~s}$ (for samples with a $T_{1} \approx 500 \mathrm{~ms}$ ). Recent work has seen improvements to the PGSERARE sequence to reduce the influence of imaging artefacts [80, 86, 87]. Additionally, numerical simulations of fluid flow within a concentric cylinder geometry have illustrated the importance of selecting appropriate NMR parameters to reduce errors caused by fluid flow over an NMR velocimetry experiment [86, 87].

Furthermore, NMR measurements on materials under shear have utilised various nuclei. Proton $\left({ }^{1} \mathrm{H}\right) \mathrm{NMR}$ remains the most commonly studied nucleus and deuterium $\left({ }^{2} \mathrm{H}\right)$ spectroscopy was described in Section 3.4.2 as a tool for probing order parameters. Additionally, a recent study has shown that the nuclear electric quadrupolar coupling of ${ }^{23} \mathrm{Na}$ can be used to determine molecular alignment in fluids of biological origin where sodium ions are found naturally [88].

\footnotetext{
${ }^{1}$ Utilising spiral $k$-space sampling and compressed sensing techniques a recent publication presented results in which NMR velocity maps were acquired at a rate of 188 frames per second [85].
} 


\subsection{Hardware (Drag Flow Shear)}

This section focuses on drag flow shear geometries which have been developed for various NMR systems. For investigating materials under shear with NMR the vast majority of experiment set-ups utilise drag flow shear devices (e.g. concentric cylinder and cone-plate) and thus warrant specific discussion. Later in this chapter other types of hardware are discussed including extensional flow shear devices, mechanical strain wave generators, permanent magnet NMR spectrometers and magnetic field gradient sets.

\subsubsection{Commercially Available}

Currently the only commercially available Rheo-NMR hardware is produced by Magritek Ltd. and distributed by Bruker Biospin GmbH (Figure 4.1). A description of this kit can be found in the "2001 Bruker Annual Report" [89]. In general the system consists of three main pieces: 1) a stepper motor and gearbox, 2) a drive-shaft and 3) a shear geometry. With this kit the shear geometry is mounted to the resonator coil and loaded from underneath into the NMR magnet. Next, the drive-shaft is lowered into the magnet from above and coupled to the shear cell via a bayonet style connection. Finally, the motor rig is installed onto the magnet and using an Oldham style coupling the motor/gearbox assembly is connected to the drive-shaft. This system operates in strain rate mode and the revolution of the motor can be controlled by a front panel interface or NMR spectrometer TTL outputs. Furthermore, complex motor operations can be accomplished through a RS232 connection which allows for single line motion commands as specified in the Rheo-NMR User Manual (v3.1.1) [90].

\subsubsection{Unique, Custom and One-Off}

Examples of home-built shear hardware exist in the literature [e.g. 81, 91, 92] and attention will now be given to some notable instances. This includes examples in which torque measurements were utilised within a commercial NMR system. One of the most exciting instruments was presented by D.A. Grabowski and C. Schmidt [91] in 1994. In this manuscript a set-up was described in which a cone and plate geometry $\left(5^{\circ}\right)$ could shear samples within the NMR magnet while torque measurements were made simultaneously via a torsion element and an optical sensor. A maximum torque was not listed for the system but calibration curves showed that viscosities could be measured in the range of $500-4000 \mathrm{Pas}$. As a strain rate controlled device it was said to operate between 0.1 to $100 \mathrm{~s}^{-1}$ in a range of $5-100^{\circ} \mathrm{C}$ using a modified Bruker temperature regulator. In this communication (and a subsequent publication [93]), ${ }^{2} \mathrm{H}$ spectroscopy 


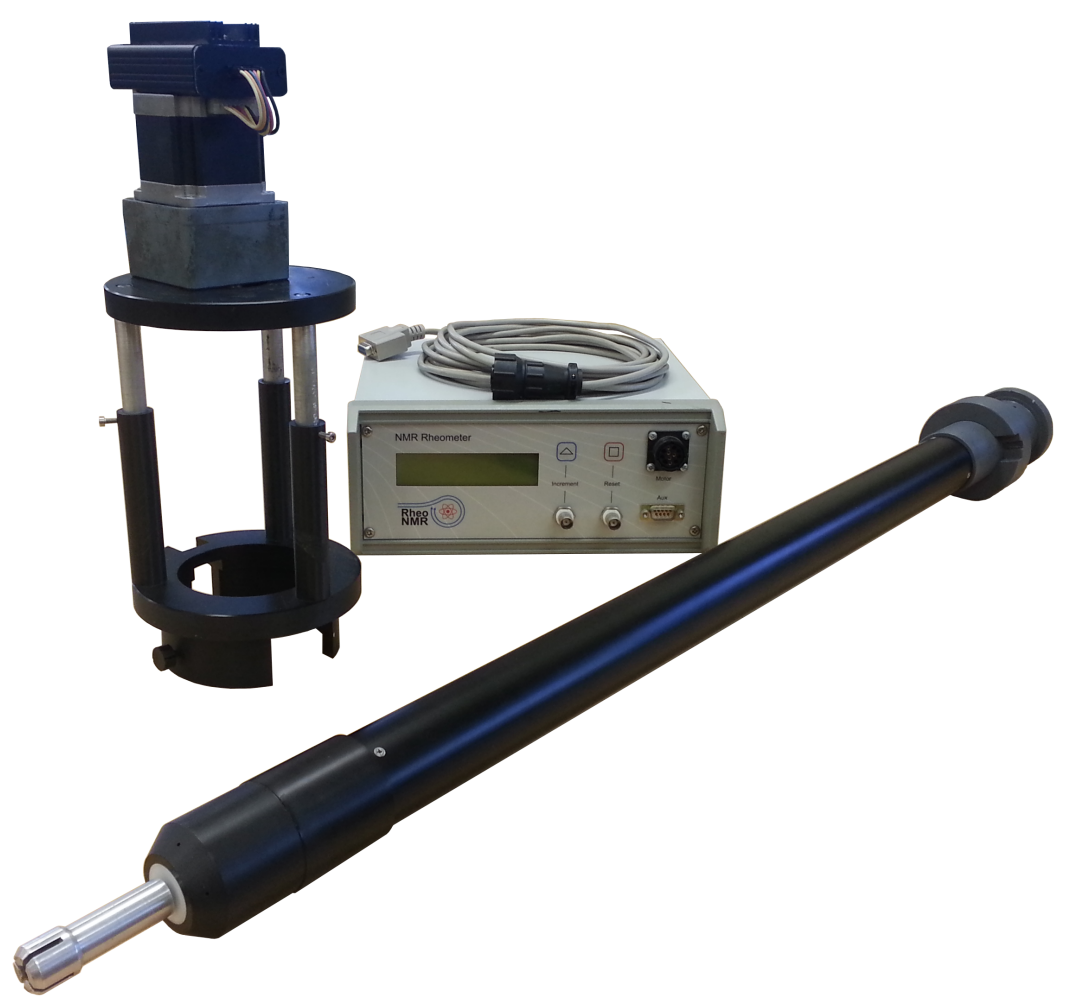

(a)

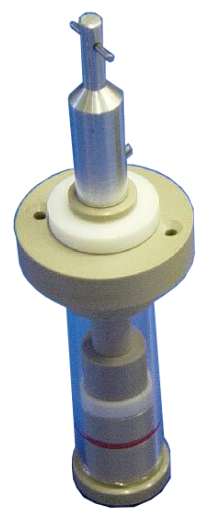

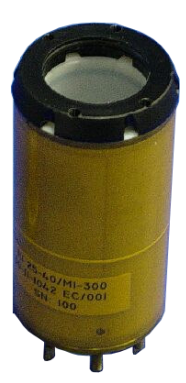

(b)

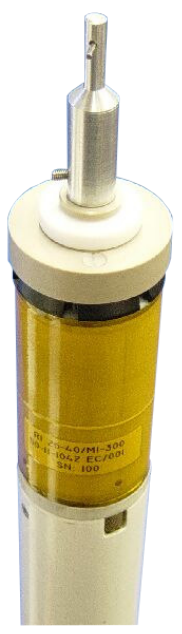

(c)

Figure 4.1: Components of Magritek Rheo-NMR kit. (a; left) Motor, gearbox and mount, (a; right) drive-shaft and (a; centre) controller module. (b; left) Standard parallel disks shear geometery and (b; right) $25 \mathrm{~mm}$ RF coil for Bruker Micro2.5 imaging probe. (c) Shear device mounted in RF coil. Note: Figures $4.1 \mathrm{~b} \& 4$.1c have been modified from original images in the Rheo-NMR Manual (V3.1.1) [90]. 
data was presented with simultaneous torque measurements. Although the cone and plate device has been used in other publications, torque data is not presented outside of the initial two manuscripts.

Another attempt to couple a torque sensor to NMR measurements can be found in an article from J.S. Raynaud and colleagues [81] in 2002. In this manuscript a torque sensor was placed inline with the drive-shaft and was capable of measuring a max torque of $0.7 \mathrm{~N} \mathrm{~m}$; however, due to "significant and fluctuating residual friction at the bearing" the resolution was limited to $10 \%$ of the full range torque. As the torque required to shear the sample (bentonite suspension) was on the order of $0.004 \mathrm{~N} \mathrm{~m}$, no data were presented from simultaneous torque and NMR experiments.

In October 2014 a colloquium, Workshop on Rheo-NMR, was held at Karlsruhe Institute of Technology (Karlsruhe, Germany) where aspects of this thesis were presented in an oral contribution. In response to this talk, a fellow speaker (U. Scheler) reported that their group had also constructed a Rheo-NMR instrument which could simultaneously measure torque during the NMR experiment. This aspect of the hardware and subsequent data were never published; however, custom hardware from this group was published in which polymer melts were studied via NMR relaxation at temperatures up to $190^{\circ} \mathrm{C}$ within a commercial magnet [94].

Finally, simultaneous NMR and bulk rheological measurements have been realised by the research group of $\mathrm{M}$. Wilhelm where commercial rheometers have been outfitted with home-built NMR systems (a reciprocal approach to those previously mentioned). As these systems utilise permanent magnets, further discussion of this work can be found in Section 4.4.2.

\subsection{Emerging Techniques}

This section explores hardware and techniques in which magnetic resonance is used to investigate rheological properties; however, these methods, historically, have not been classified as Rheo-NMR.

\subsubsection{Magnetic Resonance Elastography}

First purposed by R. Muthupillai and colleagues [95] in 1995, magnetic resonance elastography (MRE) combines magnetic resonance imaging with mechanically imposed harmonic strain waves to measure rheological parameters such as shear modulus (a review of the technique is available [96]). MRE provides for local rheological measurements, a key feature for studying heterogeneous materials such as biological tissue, where the technique is commonly applied. 
In developing magnetic resonance elastography for the medical community phantoms of various gels have been studied. In addition, a $\mu \mathrm{MRE}$ set-up has been constructed for a vertical bore micro-imaging system [97]. However, despite the potential for laboratory material science applications, MRE has been developed primarily as a non-invasive and in situ diagnostic tool in medicine.

\subsubsection{Permanent Magnet NMR Rheology}

The footprint, costs and expertise needed to maintain a superconducting NMR system are a few barriers which will continue to slow the growth of the Rheo-NMR community. In general these factors have globally been recognised as limits to the fields of high-resolution NMR spectroscopy, diffusion and micro-imaging. In response, a recent trend can be seen in commercially available hardware that utilise portable/bench-top permanent magnet set-ups, some of these have user-friendly "push-button" interfaces with pre-defined NMR methods. Examples include, among others: the Magritek Spinsolve, the NMReady Nanalysis and the Thermo Scientific picoSpin (for spectroscopy) and the Magritek Benchtop MRI Tomograph, the Magritek Rock Core Analyzer, the Bruker ICON and the Oxford Instruments GeoSpec2+ (for imaging and diffusion).

One such device is the NMR-MOUSE from Magritek. The NMR-MOUSE [98] is a single sided NMR magnet which has continued to evolve since originally being introduced in 1996. One iteration of this system utilised the strongly inhomogeneous $B_{0}$ and $B_{1}$ fields in addition to planar gradient coils to measure velocity propagators in laminar flow in a thin rectangular pipe [99]. These techniques were later extended to 2D velocity imaging with slice selection to measure flow in a circular pipe [100]. As pointed out in both articles, the magnet geometry of the NMR-MOUSE enables ex situ applications where flow could be measured on systems not compatible with the confines of a traditional closed magnet. Other work with the NMR-MOUSE has demonstrated the potential to monitor the curing of rubber [101] and recently a prototype portable NMR spectrometer [102] $(<1 \mathrm{~kg})$ illustrates these instruments are well suited for industrial and other non-traditional applications. Beyond the NMR-MOUSE there is a growing interest in permanent magnet systems for industrial process and quality control [103]. While this hardware is related to Rheo-NMR it does not solely focus on materials under shear and is thus outside the scope of this review. As a low cost instrument with a growing user base and the possibility to measure flow and diffusion along with NMR relaxometry, the NMR-MOUSE would be well suited for the studies of materials under shear in both the laboratory and the industrial environment.

Work with superconducting magnet systems has shown the possibility of measuring viscosity in steady pipe flow with MRI techniques [104, and references therein]. This strategy has now been implemented in a commercially available permanent mag- 
net instrument: the Flowscan system from Aspect Imaging. It delivers real-time rheological measurements including: viscosity, apparent yield stress, apparent slip velocity, phase separation and turbulence over time [105]. By utilising NMR methods, the online fluid characterisations are non-contact and thus are well suited to a wide range of industrial applications.

As an alternative to the methods mentioned above, where spatially resolved flow profiles are used to calculate viscosity, early work by N. Bloembergen, E.M. Purcell and R.V. Pound [61] suggested viscosity could be estimated from NMR relaxation data. Well logging NMR techniques in the petrochemical industry, which utilise permanent magnet systems and operate in the down hole environment, apply this concept for the rapid prediction of fluid viscosity in situ [106]. Furthermore, numerical methods (Principle Component Analysis and Partial Least Squares Regression) can be used to predict the viscosity of crude oils based upon NMR measurements and an existing catalog of characteristic measurements [107].

Finally, special attention is given to the work of M. Wilhelm and colleagues who have developed Halbach permanent magnet systems which couple to commercial rheometers [108]. Recently their home-built NMR system $\left({ }^{1} \mathrm{H}\right.$ Larmor frequency approximately $29 \mathrm{MHz}$ ) was coupled to a TA Instruments Ares rheometer [109]. For the experimental set-up a custom cone-plate geometry was used with a diameter of $13 \mathrm{~mm}$ and an angle of $12^{\circ}$. To characterise the influence of filler material on polymer crystallisation $T_{2}$ was measured as a function of time during simultaneous measurements of $G^{\prime}$ and $G^{\prime \prime}$ via small angle oscillatory shear profiles. Furthermore, steady shear experiments were performed in which simultaneous measures were made of $T_{1}, T_{2}$, viscosity and normal forces. In general, this recent work (2014) opens up an exciting new branch of material investigation in which macroscopic mechanical properties are measured in tandem with microscopic molecular dynamics. However, currently, spectroscopy and spatially resolved measurements are not yet possible.

\subsubsection{Extensional Shear NMR}

Despite the prevalence of extensional deformations in numerous processing operations and the direct impact of extensional flow on the final properties of polymer products [1, chap. 7], the majority of rheological experiments conducted within the confines of an NMR magnet utilise either drag flow or pressure driven shear geometries. In general, extensional rheology measurements are difficult, in particular for low viscosity materials. Although bulk rheological parameters (e.g. extensional viscosity) is not measured, various devices have been used to study extensional shear with NMR, including the four-roll mill [75, 110], cross jet [75] and semi-hyperbolic converging die [111] geometries 
Both the four-roll mill or cross jet geometries generate stagnation flows [1, sec. 7.7]. In both cases, the flow is not homogeneous and shear stresses are generated at the surfaces of the geometries. However, in the centre of the flow, at the stagnation point, it is possible to generate strong extensional strains. As a result, for samples comprised of polymer molecules or other asymmetrical particles, at the stagnation point there may be a high degree of orientation detectable by NMR measurements.

For pressure driven flow through an axisymmetric channel, it is possible to determine a channel profile such that the velocity at the centre of the channel increases linearly and thus generates a constant extensional shear rate [112]. Utilising a semihyperbolic profile, this approach has been realised for NMR velocimetry and spectroscopy measurements [111].

\subsubsection{Pulsed Second Order Field NMR}

As described in Section 3.6.3 successive experiments at varying gradients strengths can be used to measure translation displacements (diffusive and flow). In the case of homogeneous samples with coherent flow recent work [113-118] has shown that a spatially dependent pulsed gradient field (second order gradient field) can be used to simultaneously sample a range of gradient strengths. By combining traditional imaging gradients and a second order encoding field it has been demonstrated that single acquisition NMR experiments are capable of capturing an average propagator [116, 118] and thus yield a description of flow behaviour in real-time. Furthermore, as currently implemented, the measured NMR signal (echo) is the characteristic function (averaged propagator), eliminating the need for complex post-processing. As suggested by W.C. Kittler, M.W. Hunter and P. Galvosas [118] future applications of this technique could include online monitoring of batch reactions via measurements of the material under flow.

\subsection{Conclusion}

In short, Rheo-NMR is a diverse field and it is reasonable to expect further development and applications in academia and industry as new NMR instruments become portable, cheaper and easier to use.

To continue advancing the field of Rheo-NMR, the motivation of this thesis was to close the gap between NMR experiments and traditional rheometry. To this end the experimental work in the following chapters will contribute to the following three key areas:

\section{Improved shear geometries for Rheo-NMR}


- Maintain "ideal assumptions"

- Consideration for temperature control

\section{New Rheo-NMR experimental protocols and control}

- Steady, step and oscillatory strain waveforms

- Interaction between NMR acquisition and deformation

\section{Simultaneous bulk rheometry during Rheo-NMR experiment}

To set the stage for the remainder of this thesis, the present chapter has introduced various work from the past three decades which has utilised NMR techniques to investigate material properties during deformation or to infer a rheological parameter. As is relevant for this thesis on methods development, this literature review focused mainly on the instruments developed and their implementation in previous studies. Furthermore, techniques were explored from very different disciplines, ranging from diagnostic medicine to down-bore petrochemistry. Magnetic resonance measurements of materials under shear have included both spatially resolved and bulk spectroscopy, relaxation and flow encoding. Where systems based on both superconducting and permanent magnets have been utilised. 


\section{Chapter 5}

\section{Instrument Design and Control Software}

\subsection{Introduction}

The following chapter explores the design and construction of various new pieces of Rheo-NMR hardware including: drag flow shear geometries, drive-shaft units, electronics and software. Individually each piece is only a component of a Rheo-NMR experiment and needs to be combined with other parts to enable an NMR experiment. In particular the control software can be used for numerous shear geometries and driveshafts although not all functions would be utilised by any one configuration. For simplicity this chapter is sub-divided into the individual projects; however, to keep the respective big picture and end goal in mind, below three themes are introduced. In reading through the chapter these concepts will be revisited to help understand how an individual part contributes to the new methods developed in this thesis.

\subsubsection{Improving Shear Geometries}

One goal of this thesis was to not only develop NMR geometries that resemble their rheometry counterparts, but provide experimentalists with the ability to investigate assumptions of ideal geometries in their studies. As such, a scaleable concentric cylinder design was developed so that the influence of curvature could be explored. Additionally, the length of this geometry could be easily changed to explore end effects or the surface of the rotor modified to explore wall slip. Furthermore, a parallel disks geometry was built where the side of the sample could either be a fluid-air boundary or immersed in a sea of fluid. Additionally, the gap in this parallel disks set-up could be altered and by replacing one surface, a cone and plate geometry could be realised. This strategy allows for Rheo-NMR experiments which closely resemble traditional rheom- 
etry (e.g. concentric cylinders with negligible curvature, cone and plate that meet the small angle approximation, similar boundary conditions) or specifically address an experimental parameter (e.g. wall roughness, curvature).

Although methods were developed in this thesis such that bulk rheometry measurements could be made during the NMR experiment, shear devices were constructed for a commercial rheomter (AR2000, TA Instruments). Thus the design and construction of new shear devices (e.g. materials, dimensions) could be evaluated with conventional techniques.

\subsubsection{New Experimental Protocols}

The Rheo-NMR kit produced by Magritek and distributed by Bruker Biospin was previously introduced in Section 4.3.1 with the core hardware shown in Figure 4.1a. The shear cells sold with this kit are stabilised within the resonator coil through a concentric fit between the geometry and the NMR machine. Mounting cells in this configuration impedes the flow of gas over the sample which is part of the temperature control strategy for the Bruker hardware. Additionally, while explicit commands can be sent to the motor via a serial command line, it would be difficult to implement time dependent shear profiles without additional hardware. The only real-time control over the motor is via the TTL outputs of the spectrometer connected to the RESET and INCREMENT functions of the motor controller; these are used to stop the motor and increment the motor frequency in pre-defined steps, respectively.

In this chapter, a new concentric cylinder design is presented which can easily be scaled for various magnet geometries and is compatible with the Bruker temperature control system. Incorporating sinusoidal shear waveforms required a new motion drive system and a redesigned mechanical coupling to eliminate the backlash inherent in the bayonet connection used to connect the drive-shaft to the shear cell in the currently available Rheo-NMR kit. Furthermore, to synchronise the time dependent shear waveform with the NMR acquisition, a digital interface has been developed between the motor electronics and the existing TTL outputs and trigger inputs of the Bruker spectrometer. Programmed into the motor firmware are options for: 1) the motor to trigger the spectrometer (due to timing or position) or 2) the NMR system to trigger specific conditions on the motor (e.g motion start, stop, increase or decrease). This feature is particularly useful for oscillatory shear in which subsequent NMR experiments can be executed at the same position in the sinusoidal profile but could easily be adapted for other applications. 


\subsubsection{Simultaneous Bulk Rheometry}

A third goal of this thesis was to combine bulk rheology measurements with NMR techniques. Utilising a strain rate controlled instrument (as has been typical of RheoNMR to date) this meant the inclusion of a torque measurement in order to calculate the bulk shear stress response. As mentioned in the previous chapter (Section 4.3.2) this concept has been applied previously, however, limited results exist in the current literature.

To measure torque two approaches were used: 1) an estimate from the current necessary to drive a servo-motor and 2) an analogue inline sensor was added to the driveshaft unit. In addition to mechanically integrating this hardware, software and auxiliary electronics were needed to calibrate, sample and store the torque measurements.

\subsection{Shear Geometries}

As part of this thesis, a number of shear cells have been designed and constructed. The types of geometries include concentric cylinders, parallel disks, and a hybrid planarcylindrical Couette device. Included in this section are discussions including: 1) intended use, 2) selection of materials (NMR safe and machinability) and 3) mechanical design. Exploded view diagrams for each of the devices discussed in this section can be found in Appendix B.

\subsubsection{Concentric Cylinder}

A key feature of the new concentric cylinder design is the provision for air flow between the device and the inside bore of the resonator (Figure 5.1a). This void allows the shear cell to be used in conjunction with a temperature control unit (e.g. BVT 3000) which utilises the flow of temperature regulated gas (typically dry compressed air or $\mathrm{N}_{2}$ gas) to maintain a set point temperature within the resonator. Viscosity is extremely sensitive to temperature; for example, in the case of water, the viscosity can vary as much as $3 \%$ for a $1^{\circ} \mathrm{C}$ change in temperature [6, sec. 2.2.2]. In general, higher viscosity fluids have a greater dependence on temperature making temperature control an important constraint of any rheological experiment [6]. The annular air jacket allows for the flow of temperature regulating gas where the use of glass tubing (rather than PEEK) for the outer surface of the concentric cylinder shear cell allows for greater dissipation of heat (assuming similar wall thickness and temperature gradient) from the sample due to glass having a higher coefficient of thermal conductivity $\left(1.1 \mathrm{~W} \mathrm{~m}^{-1} \mathrm{~K}^{-1}\right.$ at $0^{\circ} \mathrm{C}$ for borosilicate glass [119, sec. 12] versus $0.25 \mathrm{~W} \mathrm{~m}^{-1} \mathrm{~K}^{-1}$ at $23^{\circ} \mathrm{C}$ for unfilled PEEK $[120,121])$. Thermal conductivity of the cell material is important to both heat the 
sample above ambient conditions and/or remove excess energy generated by viscous heating.

Furthermore, the concentric cylinder design presented above was modified for use with the new backlash free drive-shaft (presented in Section 5.3.1). For this variant the resonator coil mount pieces were unnecessary as the shear device coupled directly to the drive-shaft housing. To attach the cell to the drive-shaft housing a threaded connection is used and the top cap in the assembly above (seen in Figures 5.1b and 5.2) is replaced by a version with male threads.

\section{Variable Temperature}

Due to the fit between the end caps and glass tubing of the concentric cylinder device and the difference in the coefficient of thermal expansion between the PEEK and glass materials $\left(3-5.5 \times 10^{-6} \mathrm{~K}^{-1}\right.$ for borosilicate glass [122] versus $1.7-4.8 \times 10^{-5} \mathrm{~K}^{-1}$ for unfilled PEEK $[120,121])$, an increase of temperature within a reasonable experimental range $\left(20-65^{\circ} \mathrm{C}\right)$ has the potential to crack the precision bore glass tubing.

The issue with stressing the glass tubing at the two ends is further exacerbated by the process of cutting the glass to length. In cutting glass small micro-fractures are formed which, left untreated, can lead to significant cracking. A common solution to this issue is to heat the freshly cut glass ends (e.g. flaming the glass), however, while this approach seals any cracks it also deforms the inner bore of the tubing. As the design presented required pieces to be machined to fit within the tubing heat sealing the tubing ends was not an option. As an alternative the glass tube lengths were over cut and then precision ground to length. The grinding operation had less potential to form micro-fractures and made the heat treatment unnecessary.

Despite the approach to treating the ends of the glass, as discussed above, the thermal expansion of the PEEK plastic pieces within the glass tubing frequently lead to failure. To combat this problem the plastic material was replaced with a machinable ceramic (i.e. $\mathrm{MACOR}^{\circledR}$, Corning Incorporated [123]). This ceramic was chosen as the coefficient of thermal expansion $\left(8.1 \times 10^{-6} \mathrm{~K}^{-1}\right.$ [123]) closely matched that of the borosilicate glass tubing. Thus regardless of the tolerance chosen between the end caps and the tubing, as the shear device is heated or cooled, the fit should be maintained. Due to material costs and considerations for wear (part lifetime) MACOR is not suggested to be a direct replacement of PEEK but would be used in the case of Rheo-NMR experiments conducted at temperatures $>30^{\circ} \mathrm{C}$. 


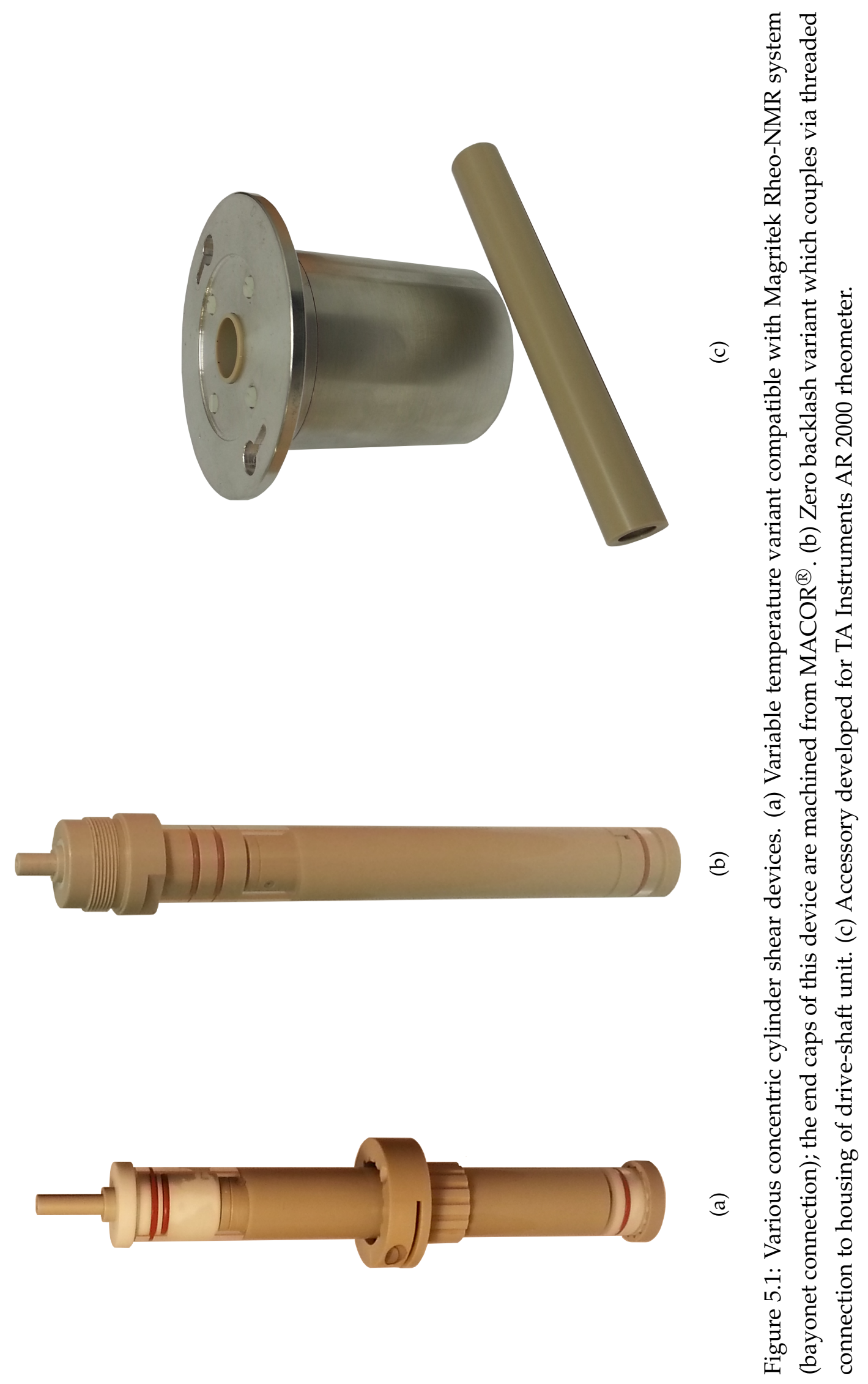



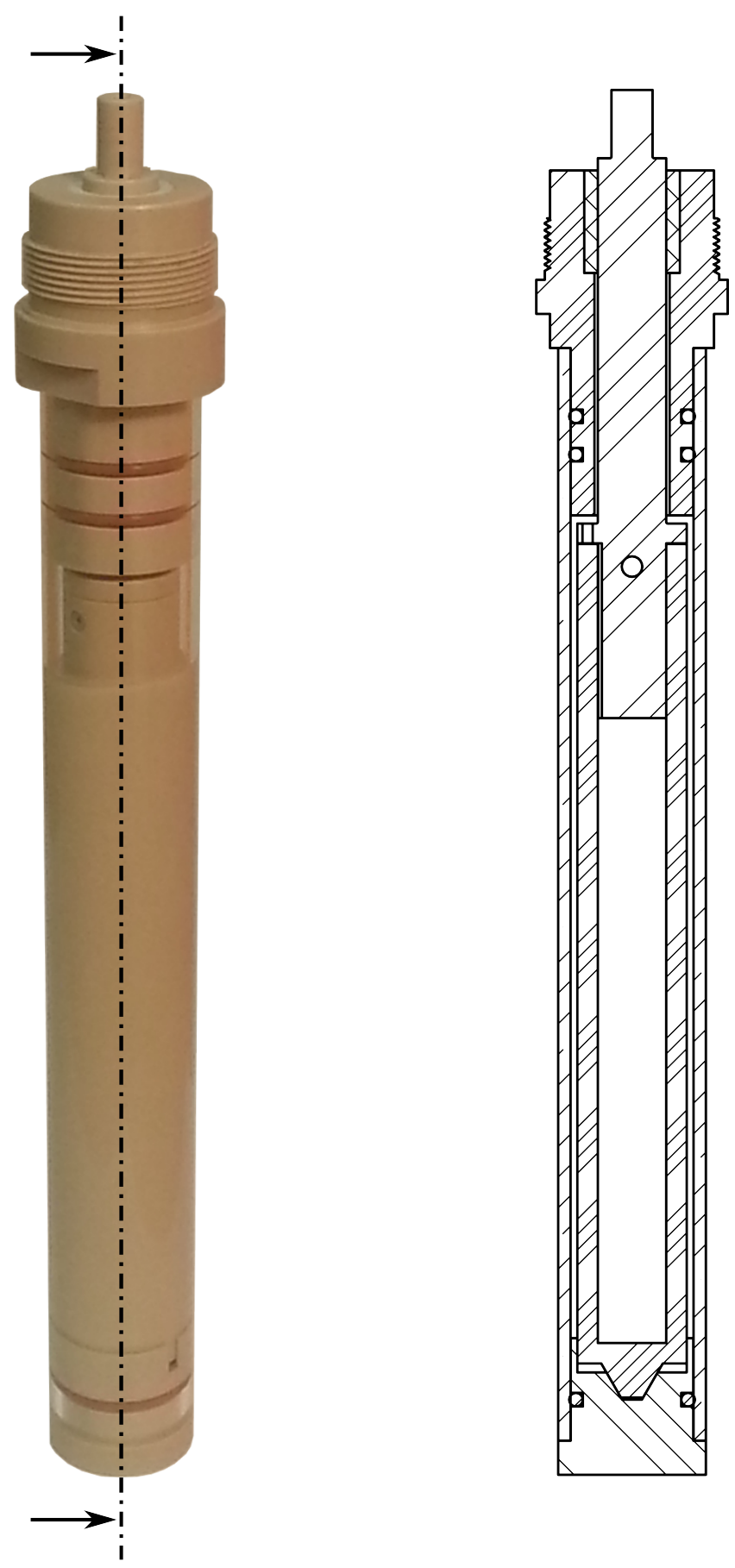

Figure 5.2: Concentric cylinder device built for new drive-shaft unit with cross section. 


\section{Super Wide Bore}

In Section 2.4.2 the appropriate tools were used to develop a description of the velocity field, shear rate and shear stress in the case of the concentric cylinder geometry. In particular it was noted that when $r_{\mathrm{o}} \approx r_{\mathrm{i}}$ the geometry can be treated the same as simple shear between two infinite parallel plates (Equation 2.121). To compare the radii of surfaces, a dimensionless constant $\kappa$ can be used [1]

$$
\kappa=\frac{r_{\mathrm{i}}}{r_{\mathrm{o}}}
$$

where curvature can be ignored in the case of $\kappa \geq 0.99$.

For Bruker wide bore micro-imaging systems commercially available resonators are limited to $\leq 30 \mathrm{~mm}$. Furthermore, signal-to-noise concerns practically limit the size of imaging voxels which in turn motivates gap widths on the order of $1 \mathrm{~mm}$. Considering a geometry where $r_{\mathrm{i}}=10 \mathrm{~mm}$ and $r_{\mathrm{o}}=11 \mathrm{~mm}, \kappa=0.91$ and (for a sample with Newtonian behaviour) the shear stress increases by more than $20 \%$ across the gap (Equation 2.110). In this case the geometry certainly does not represent simple shear between infinite plates. Depending on the fluid under investigation and desired comparisons to other work geometries with different $\kappa$ values may be necessary.

Utilising a Bruker super wide bore magnet samples may have diameters up to $66 \mathrm{~mm}$ allowing for larger shear cells and (for a fixed gap) lower $\kappa$ values. Such a magnet was available through collaborators in the Magnetic Resonance Microscopy Laboratory at Montana State University, Bozeman (USA). The concentric cylinder design illustrated in Figures 5.1b and 5.2 was scaled for this instrument and a number of shear cells were constructed with various interchangeable rotors (a full list of the possible combinations can be found in Table 5.1). To allow the rotors to be changed, the bottom cap was modified. Previously there was a "crown" on the cap which helped to maintain the alignment between the rotor and cup. This feature can be seen in the cross section of Figure 5.2. As the thickness of this feature was the same as the desired gap this strategy was not compatible with interchangeable rotors and was thus removed.

For a number of the possible geometries listed in Table 5.1 the $\kappa$ value is quite small $(\kappa<0.7)$ indicating the stress variation across the gap for Newtonian behaving fluids is quite large. Despite the inhomogeneous velocity field across the fluid domain these devices can be particularly useful in studying granular material. In these cases, grains can be on the order of $1 \mathrm{~mm}$ and when many grains are desired between the shear surfaces, the utility of gaps on the order of $10 \mathrm{~mm}$ is apparent.

\section{High Resolution NMR (Narrow Bore Magnet)}

Furthermore, the concentric cylinder design could be scaled down for use with High Resolution NMR magnets. These vertical bore magnets do not include the imaging gra- 


\begin{tabular}{|c|c|c|c|c|}
\hline & \multicolumn{4}{|c|}{ cup radii /mm } \\
\hline & 9.00 & 12.50 & 17.00 & 23.65 \\
\hline 6.35 & $2.65(0.71)$ & $6.15(0.51)$ & $10.65(0.37)$ & $17.30(0.27)$ \\
\hline 7.10 & $1.90(0.79)$ & $5.40(0.57)$ & $9.90(0.42)$ & $16.55(0.30)$ \\
\hline 7.60 & $1.40(0.84)$ & $4.90(0.61)$ & $9.40(0.45)$ & $16.05(0.32)$ \\
\hline 8.00 & $1.00(0.89)$ & $4.50(0.64)$ & $9.00(0.47)$ & $15.65(0.34)$ \\
\hline 11.10 & - & $1.40(0.89)$ & $5.90(0.65)$ & $12.55(0.47)$ \\
\hline 11.50 & - & $1.00(0.92)$ & $5.50(0.68)$ & $12.15(0.49)$ \\
\hline 15.10 & - & - & $1.90(0.89)$ & $8.55(0.64)$ \\
\hline 16.00 & - & - & $1.00(0.94)$ & $7.65(0.68)$ \\
\hline 21.00 & - & - & - & $2.65(0.89)$ \\
\hline 22.65 & - & - & - & $1.00(0.96)$ \\
\hline
\end{tabular}

Table 5.1: Concentric cylinder shear cell combinations available with constructed components. Listed in the table is the gap and in parenthesis the $\kappa$ value of a Newtonian fluid for each rotor and cup pair.

dients and are primarily used for NMR spectroscopy. The resonators on these systems have a maximum sample diameter of $5 \mathrm{~mm}$. Previous designs for concentric cylinder geometries used an NMR tube (outer diameter $\approx 3 \mathrm{~mm}$ ) placed inside another tube ( $5 \mathrm{~mm}$ outer diameter). At the top of the device a plastic spacer was inserted into the $5 \mathrm{~mm}$ tube to concentrically align the inner cylinder. At the base the tube alignment relied on the curvature of the tubes. Actual performance of this design would vary greatly depending on variation between the NMR tubes.

In an updated design the bottom of the outer $(5 \mathrm{~mm})$ tube is removed and replaced by a machined PEEK insert. The insert is produced to fit the inner diameter of the outer tube while a pocket was machined to match the outer diameter of the inner tube. With this design the alignment of the cell no longer depends on the arbitrary curvature of the bottom of the tubes but instead the diameters of the cells. Currently, modified NMR tubes are used for the two surfaces but they could be replaced with precision bore glass tubing or injection molded PEEK parts.

An additional benefit of using an open tube for the outer surface (i.e. cup) is that loading high viscosity samples is much easier. Instead of waiting for the sample to flow to the bottom of the tube the open geometry could be used as a pipette with a suction bulb drawing the sample directly into the bottom of the tube. 


\section{Commercial Rheometer}

In an effort to bridge traditional rheometry techniques with NMR methods a custom concentric cylinder accessory (Figure 5.1c) was built for a TA Instruments AR2000 rheometer (Figure 2.17b) such that bulk rheometry experiments could be conducted in a shear cell with the same dimensions and materials as are used within the NMR magnet. A three piece aluminium adaptor was machined to align a glass or PEEK tube (the cup of a concentric cylinder) within the "AR Series Smart Swap Concentric Cylinder Peltier Jacket" (PN 545611.901, TA Instruments). Rotors were also machined from PEEK and were designed with a concentric transition fit to the rheometer motor. Furthermore, the rotors had tapped threads to match the drawbar of the rheometer for a secure attachment.

\subsubsection{Parallel Disks}

In addition to the concentric cylinder shear cells presented above a new parallel disks geometry has been developed. Included in the design are provisions to easily change the gap distance or alter the boundary condition on the side of the sample. The geometry was built to mount directly to the new style of drive-shaft (Section 5.3.1) such that oscillatory shear experiments are possible. The shear surfaces can also be mounted onto a commercial rheometer for comparison studies. Finally, one of the flat plates can be swapped out for a cone making the device a cone and plate geometry.

The overall parallel disks device can be seen in Figure 5.3. The upper piece of the housing has threads to attach to the housing of the drive-shaft unit and contains bushings and a spindle on which the upper shear surface is mounted. Utilising washers, the depth of the geometry can be modified; using plastic shim stock, washers on the order of $10 \mu \mathrm{m}$ can be realised. A machined tube is used to connect the upper portion of the geometry to the bottom where the lower shear surface is attached. Two versions of the tube are available such that the sample can be immersed in excess fluid or alternatively the edges can be cleaned. As necessary these two approaches could be used to investigate edge fractures and other influences of the boundary.

As previously mentioned the upper shear surfaces can be directly mounted onto the spindle of an AR2000 rheometer. To mount the lower surfaces, an adaptor piece was built to sit onto of the peltier stage, "AR Series Smart Swap Peltier Plate" (PN 531051.901, TA Instruments), such that the surface conditions (e.g. roughness) of the hardware could be investigated. 

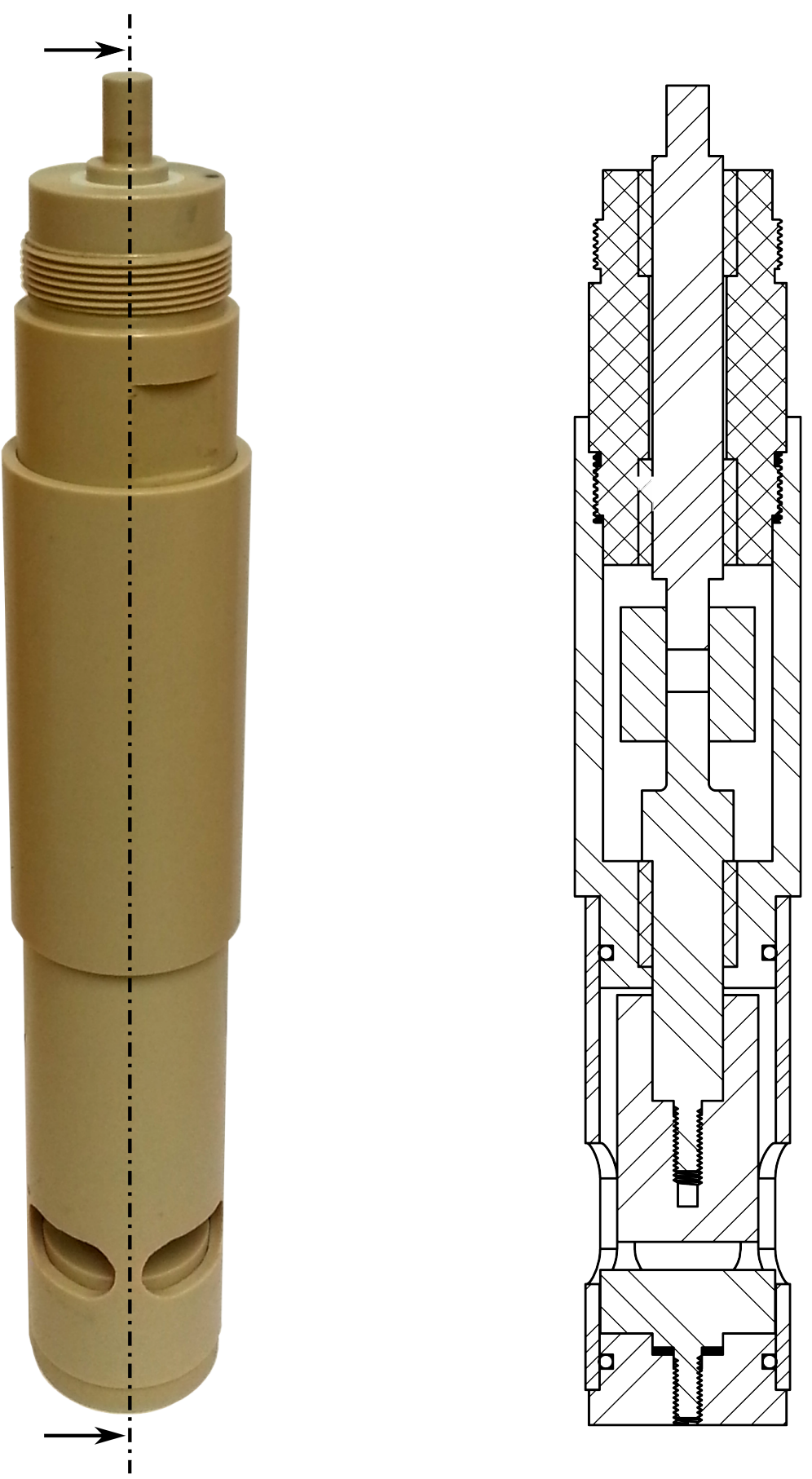

Figure 5.3: Parallel disks device built for new drive-shaft unit with cross section. 


\subsubsection{Planar-Cylindrical Hybrid}

As described previously the flow field between two concentric cylinders is an approximation of simple shear between two infinite plates. While new concentric cylinder devices allow the influence of curvature (and the resulting inhomogeneous flow field) to be reduced a geometry which eliminates curvature entirely is desirable. Experimentally a parallel plate geometry has been extremely difficult to realise for a variety of reasons [1, sec. 5.2]. Various designs have been used to visualise or measure the flow field of planar Couette flow using hot-wire anemometry [e.g. 124] and 3D particle tracking velocimetry (3D-PVT) [125]. For viscosity measurements, some analogues include the falling cylinder, band viscosmeter, falling ball, or rolling ball set-up; however, these offer little flexibility in the range of obtainable shear rates or timing of the experiment. An alternative approach is a hybrid geometry in which regions of both planar and cylindrical flow exist. Such a geometry was previously developed for laser Doppler velocimetry and computer assisted flow visualisation [126] and in this thesis was realised for NMR micro-imaging.

The original design and construction of the prototype hybrid device was carried out in tandem with the new drive-shaft design and thus the shear cell was specifically designed for use with the Magritek Rheo-NMR kit. The shear cell is mounted to the resonator and couples to the Magritek drive-shaft using the bayonet style connection. As the primary goal of the hybrid shear cell was to eliminate curvature induced stress variations across the shear gap mechanical backlash was not a concern. However, future iterations of this device could be constructed to mount onto the new drive-shaft unit.

The NMR planar-cylindrical hybrid cell (Figure 5.4a) is significantly smaller than similar devices previously reported in the fluid mechanics literature [126]. The cell was designed for a $25 \mathrm{~mm}$ resonator coil with a $1 \mathrm{~mm}$ gap between the stationary and moving surfaces. To shear the fluid two pins were used to drive a moving band similar to that of conveyor belt. These pins were constructed out of PEEK (diameter of $6 \mathrm{~mm}$ ) to prevent the pins from failing when the band was under tension. Based on the dimensions above the cell was designed to allow for the highest aspect ratio between the length of the planar section to the width of the gap.

The majority of the device was constructed from PEEK plastic, however, in the case of the band a two part silicone elastomer (Sylgard 184, Dow Corning) was produced in the lab using a custom mold (Figures $5.4 \mathrm{~b} \& 5.4 \mathrm{c}$ ). The band was cast as a cylindrical tube to prevent creating any shape memory. The dimensions of the mold were chosen to match the perimeter of the installed band with the dimension slightly modified so that the band would be under tension. During casting the two part elastomer was mixed by hand and poured into the mold. The mold was then placed in a bell jar and 


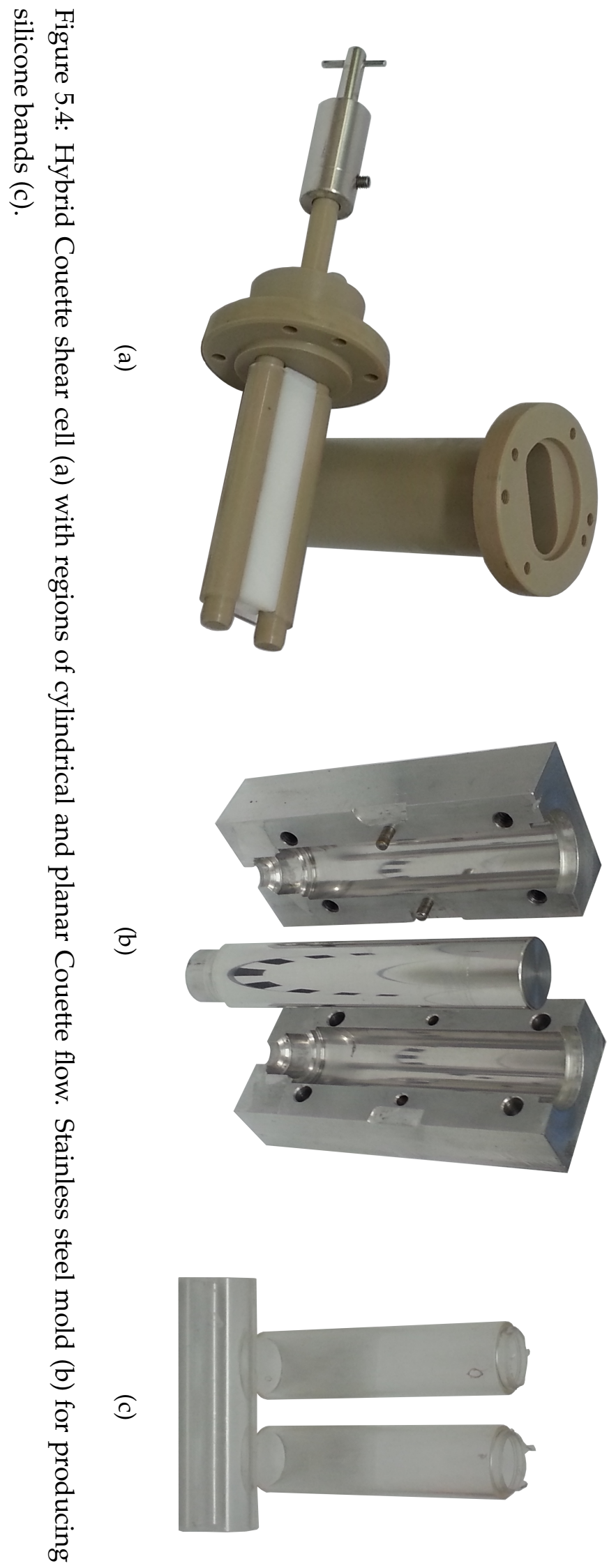


the atmosphere was removed. The process of pulling a vacuum above the mold helped to degas the elastomer and prevented bubbles from forming within the final parts. This was a particularly important step as bubbles within the band would have weakened the part and created inhomogeneities in the magnetic field due to differences in magnetic susceptibility.

Additionally, a PTFE spacer was machined to fit within the void between the pins (in the centre of the device). This piece is shown in Figure 5.4a and can be used to support the planar sections of the silicone band if necessary.

\subsection{Drive-Shaft Unit}

In this thesis the term drive-shaft is applied to two different pieces of hardware which share a generic morphology but differ in complexity. Regarding the existing Magritek Rheo-NMR kit, the drive-shaft is the component that transfers rotational motion from the motor to the shear cell; as the motor and shear device are both independent of the drive-shaft the unit is comprised of a housing, bushings and main shaft. With regards to the new hardware presented in this thesis the drive-shaft still refers to the housing and main drive component, but also the motor and shear device which are directly mounted onto the unit. The hardware presented in this section is a replacement to the existing commercially available hardware.

Within the following sections a core design will be presented which enables new Rheo-NMR experiments such as oscillatory Rheo-NMR. Subsequently, two variants of this design will be presented which either scale the hardware for use in a Bruker super wide bore magnet or incorporate an inline torque sensor.

\subsubsection{Overview (Drive-Shaft Generic)}

The drive-shaft unit developed in this thesis incorporates the shear device, drive-shaft, and motor into a single piece of hardware. A desire to eliminate backlash led to mounting the shear geometry to the drive-shaft housing outside of the magnet. Furthermore, with the new drive system (ST-23 EVA Robotics Pty Ltd [127, 128], Section 5.4.1) gearboxes were no longer necessary to access a wide range of motor frequencies $(0.01-$ $10 \mathrm{~Hz}$ ) allowing the motor to be mounted directly onto the drive-shaft as there was no need to change gearboxes between experiments. An example of an assembled driveshaft unit can be seen in Figure 5.5 [129].

The entire assembly fits concentrically within the bore of the spinner housing on a wide bore magnet. A set screw on a collar at the top of the device controls the insertion depth into the magnet; this collar also takes the weight of the unit. 

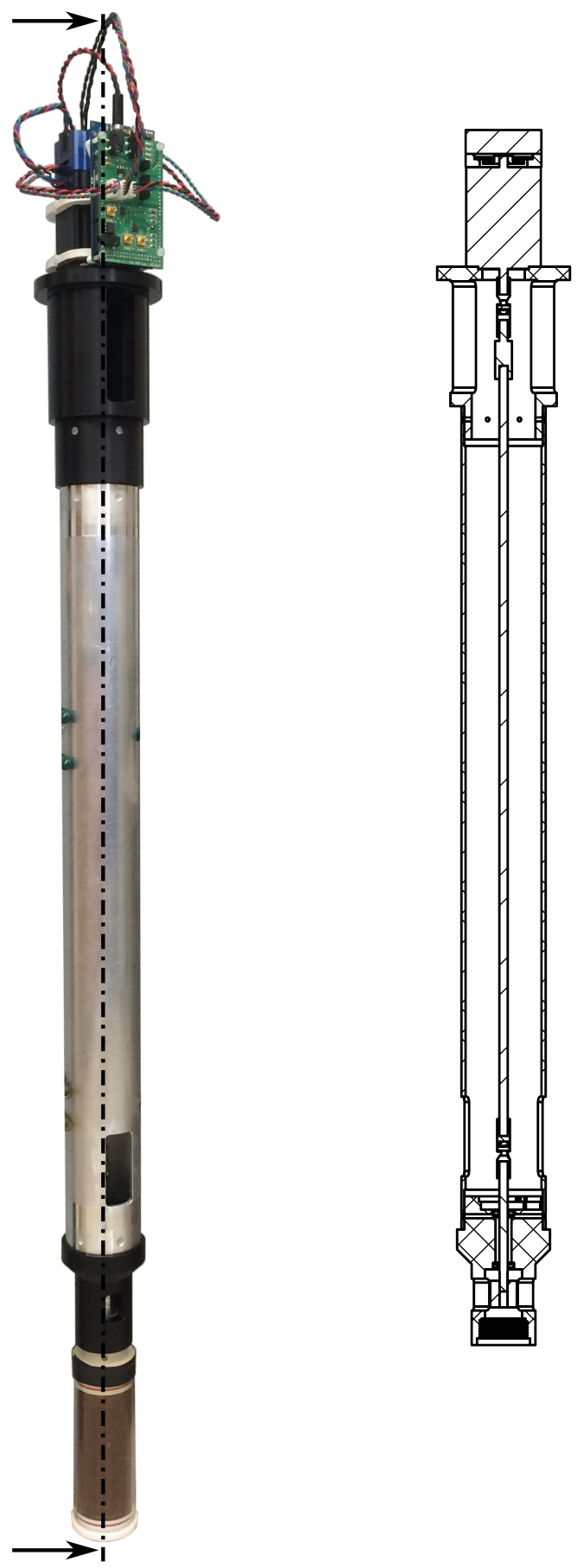

Figure 5.5: Super wide bore drive-shaft unit with cross section. 
Previously it was mentioned that the existing commercial hardware utilises a the bayonet style connection between the drive-shaft and the shear cell. While this form of a mechanical link creates undesired backlash it does allow for axial misalignment. The distance from the resonator coil to the top of the magnet is on the order of $1 \mathrm{~m}$ and even the smallest errors in the fabrication process could lead to an axial offset over the length of the assembly. Additionally, the components of the NMR system (e.g. resonator, probe) are designed for easy assembly/removal and have a generous clearance fit; thus axial misalignment on the order of $1 \mathrm{~mm}$ can be caused between the resonator coil and room temperature bore. Incorporating the shear device into the drive-shaft unit can address the issue of the resonator not being precisely aligned with the axis of the magnet, however, there is a challenge in designing and constructing a $1 \mathrm{~m}$ rotating part with negligible run-out (wobble at one end). To address this concern the rotating shaft is divided into two sections. Above the shear geometry a smaller section is mounted between two bushings or bearings so that the motion is precisely controlled. This short shaft connects directly to the rotating element of the shear geometry and attaches to the motor through a second, longer part. This longer shaft is not forced into alignment and instead utilises helical couplings or universal joints such that alignment errors can be accommodated. These couplings simplify the design as strict tolerances do not need to be maintained over the majority of the length. As the drive-shaft suspends the shear cell in the resonator coil the exact positioning of the coil is no longer important.

Additionally, the one-piece design allows for air flow around the shear cell within the resonator. This is of crucial importance when using a Bruker variable temperature controller (described when discussing the variable temperature concentric cylinder geometry). Using such a control system it is important to place the shear cell in direct contact with the temperature regulated gas, but not block the flow of temperature controlled gas. In the current design, while there are provisions for air flow around the shear cell, the bore of the magnet is blocked above the resonator. To ensure proper gas flow plumbing lines in the spinner housing part can be used to provide a conduit for gas to flow to the outside the magnet. To use this plumbing the tubing that supplies air for lift and spinning of samples, used in conjunction with the spinner housing, needs to be removed. As these features are not used during shear experiments there is no loss in functionality to the system.

\subsubsection{Super Wide Bore Variant}

The first prototype of the drive-shaft unit described in the section above was built to fit within the bore $(50 \mathrm{~mm})$ of the spinner housing on a Bruker wide bore magnet. Due to internal features of the spinner housing the shear geometries cannot have a diameter larger than $34 \mathrm{~mm}$. For the wide bore system this is reasonable as the largest commer- 
cially available resonators for the imaging system only accommodate samples up to $30 \mathrm{~mm}$. However, using a super wide bore magnet (SWB), resonators up to $66 \mathrm{~mm}$ are available allowing for larger shear geometries. A second prototype (Figure 5.5) was built specifically for a SWB by scaling up the design presented in the previous section.

An additional challenge in developing the SWB drive-shaft unit was securing the device inside the magnet. In the case of the wide bore design the assembly is concentrically aligned within the bore of the spinner housing with the weight supported by a collar outside the magnet. For the SWB no such housing was available by default. The combined shim and gradient unit is mounted within the room temperature bore $(152 \mathrm{~mm})$ with wiring and coolant lines running down from the top of the magnet. These connections prevent the drive-shaft unit from fitting concentrically within the room temperature bore. Instead an additional part was constructed to create a continuous empty bore from the top of the magnet to the resonator, analogous to the spinner housing on the wide bore magnet. To avoid fabricating a $1 \mathrm{~m}$ long NMR safe tube the upper portion of the wide bore shim system was used. The SWB at Montana State University can also function in wide bore mode by installing a second room temperature shim and gradient set; thus when performing shear experiments in the SWB configuration excess parts are available to be re-purposed. A custom end piece was machined to thread onto the upper shim barrel. With the custom piece installed the barrel would be lowered into the SWB shim/gradient system until the part came to rest on the installed resonator. The custom part had an o-ring on the exterior so that an interference fit could be established between the barrel and the shim/gradient system. Although the barrel reached the resonator, the weight of the tube (and the installed drive-shaft unit) was supported by a flange on the magnet shell which also centred the barrel along the magnet axis. An illustration of the SWB drive-shaft mounted within the magnet shell can be seen in Figure 5.6.

The upper portion of the shim barrel is a Bruker part and is constructed from at least two separate pieces threaded together. Due to the connection the drive-shaft unit could not be designed to fit concentrically within the inner bore of the tube as the bore would not be smooth across this joint. To align the drive-shaft unit the lower portion of the housing was designed to concentrically fit within the custom adaptor; the dimensions of this fit were undersized from the inner bore of the shim barrel so that the drive-shaft could easily be lowered and raised. At the top of the shim barrel a collar (fit to the outer diameter of the shim barrel) clamped onto the drive-shaft for concentric alignment and control the vertical position of the unit. 


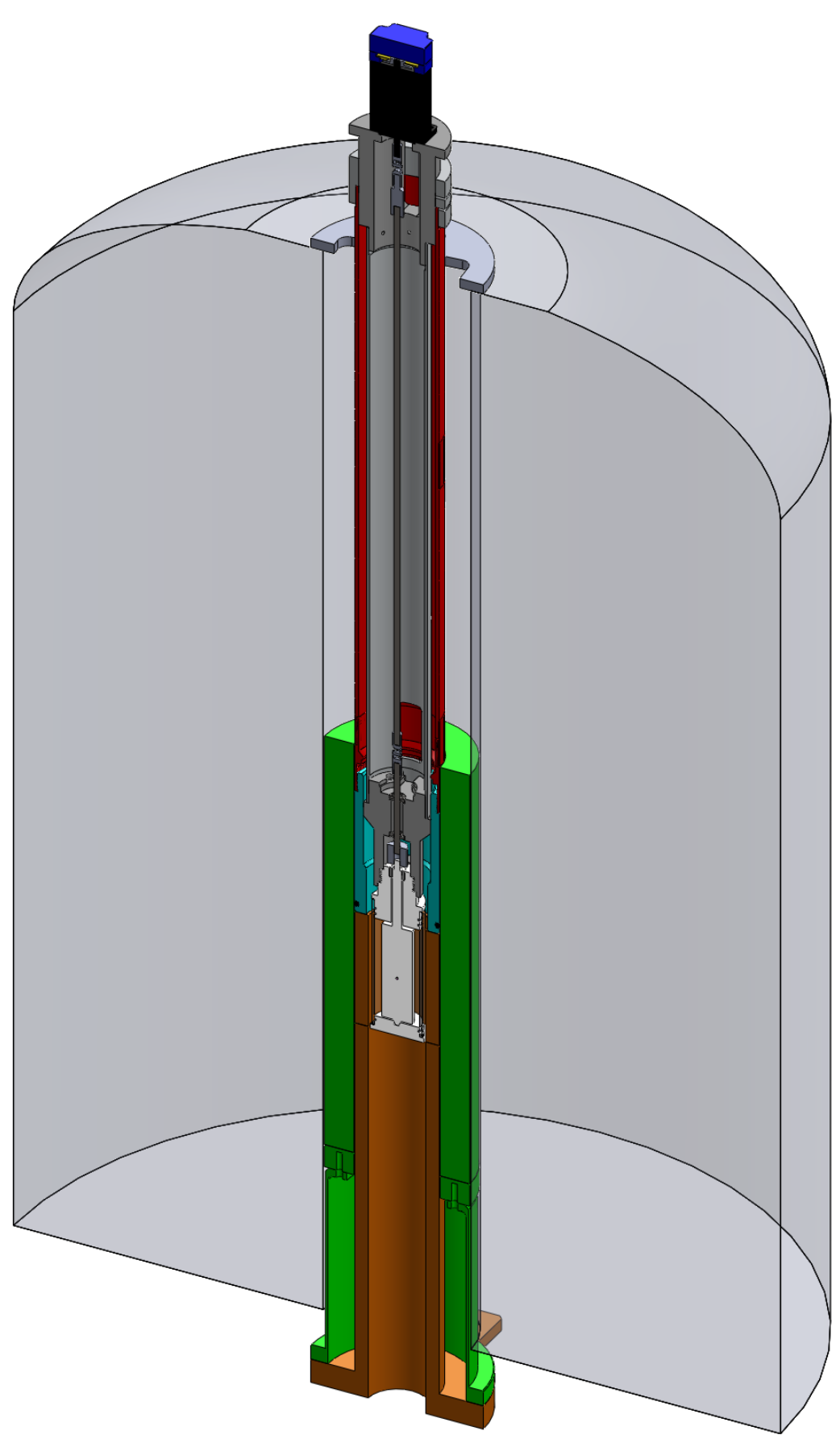

Figure 5.6: Illustration of the super wide bore drive-shaft unit mounted inside a magnet shell. The shim/gradient unit is coloured green, the probe/RF coil is coloured orange, in red is part of the wide bore shim system re-purposed for these experiments with the help of the custom built adapter shown in light blue. 


\subsubsection{Torque Sensor Variant}

An inline analogue torque sensor was incorporated into a wide bore drive-shaft unit for bulk measurements during steady shear experiments [130]. Sourcing a torque sensor with the appropriate measurement range and resolution for fluids of interest and for appropriate volumes was not a trivial task. Additionally, the sensor needed to operate correctly in the presence of a strong magnetic field and fit within the $50 \mathrm{~mm}$ bore of the spinner housing.

Ultimately, a transducer (TQ Mini 5.0) with a custom housing was sourced from Vibrac [131]. The device had a full measurement range of 5 oz in $\left(\approx 35.3 \times 10^{-3} \mathrm{~N} \mathrm{~m}\right)$ with a stated accuracy of $0.5 \%$ of the full range.

As illustrated in Figure 5.7a, the device uses two encoder style discs connected by a torsion element where the amount of light transmitted through the pair is proportional to the angular displacement and thus torque. This optical twist angle type measurement was ideal for use in the NMR magnet. After discussion with the company a sensor was built in a custom cylindrical housing ( $49 \mathrm{~mm}$ diameter) with the wiring relocated to one of the end caps (Figure 5.7b). Furthermore, the end caps were machined with tapped mounting holes.

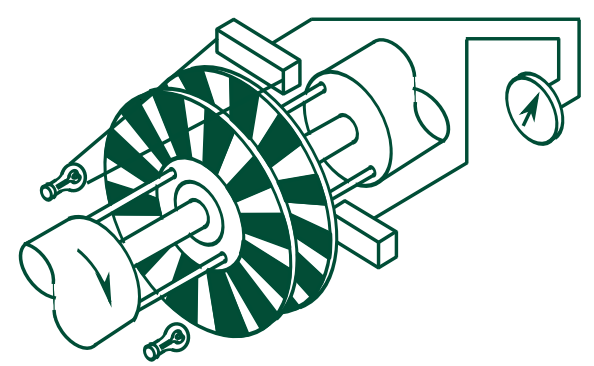

(a)

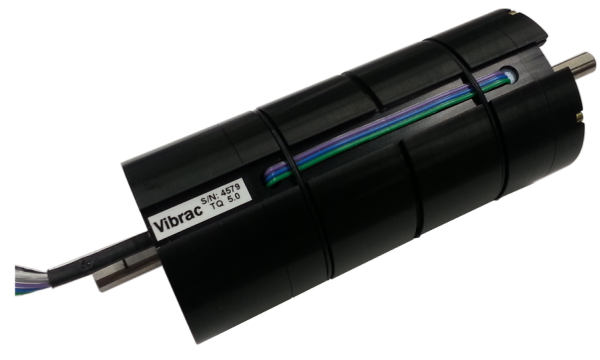

(b)

Figure 5.7: A Vibrac torque sensor was incorporated into a drive-shaft unit for bulk measurements during steady shear experiments. (a) Simple schematic to illustrate the operation of the sensor, i.e. light is transmitted through discs with an optical pattern, coupled by a torsion element, the amount of light detected relates to the angular displacement between the discs and thus torque. (b) The custom sensor built by Vibrac.

One aspect of the transducer that the vendor was not able to change was the use of carbon steel in the shaft and bearings. As these components were strongly magnetic it was not possible to fully lower the device into the magnet. During controlled experiments it was determined the transducer must be at least $300 \mathrm{~mm}$ above the resonator coil. The motor was connected to the input of the torque transducer via a long shaft and helical couplings. The sensor wiring (signal and power) was taped to the outside 


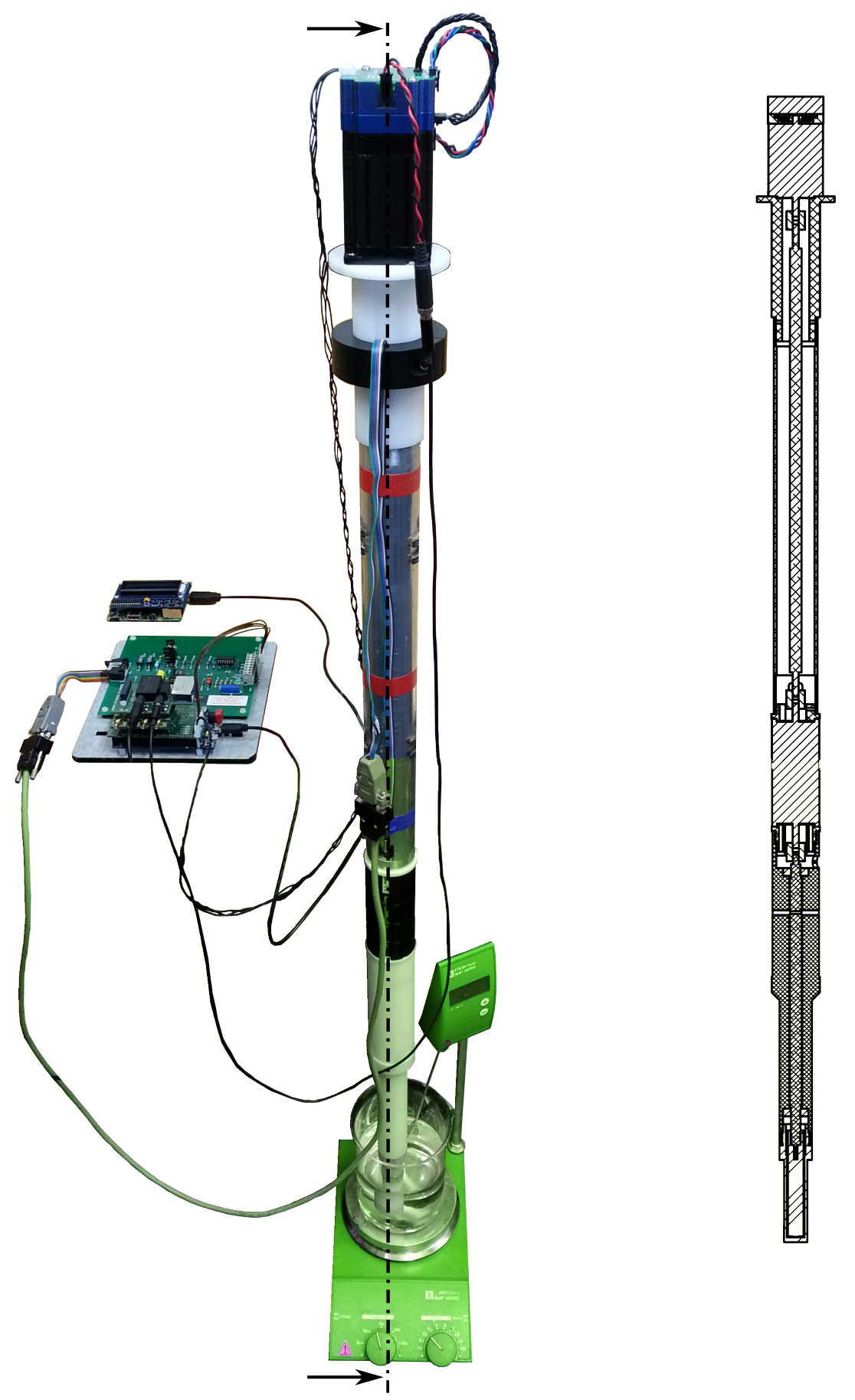

Figure 5.8: The torque variant drive-shaft photographed during bench-top calibrations with cross section. The water bath was used to control the sample temperature. 


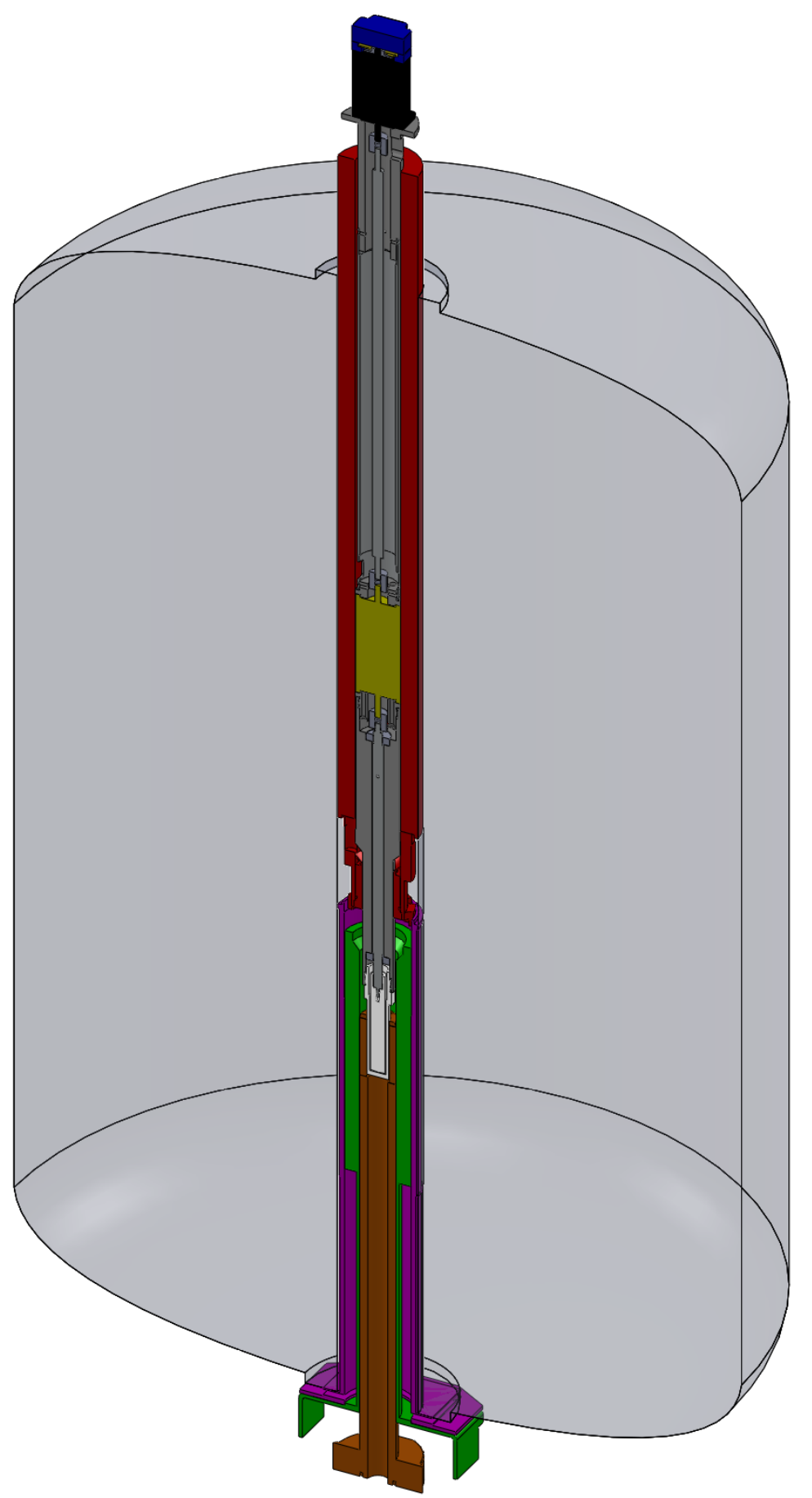

Figure 5.9: Illustration of the drive-shaft with inline torque transducer mounted within a wide bore magnet shell. The room temperature shim unit is coloured purple, the Micro2.5 gradient unit is coloured green, the probe/RF coil is coloured orange, the upper portion of the shim barrel is coloured red and the torque transducer is shown in yellow. 
housing and routed outside the magnet (supporting electronics for the transducer will be discussed in Section 5.4.3). A shaft located by two bearings connected the transducer to the shear geometry. The bearings were constructed from ceramic races and glass ball bearings as these low friction parts would not significantly influence the torque measurement; furthermore, the materials are ideal for use in a magnet.

A concentric cylinder geometry was specially built for this instrument. To prepare the device for a measurement, a rotor $\left(r_{\mathrm{i}}=6\right.$ or $\left.8 \mathrm{~mm}\right)$ was first attached directly to the drive-shaft. Next the cup $\left(r_{\mathrm{o}}=9 \mathrm{~mm}\right)$, loaded with a sample, was pushed onto the rotor and connected to the housing of the drive-shaft unit via matching threads. The rotor was attached using a location transition fit with M2 screws to clamp onto the shaft; a threaded connection was tested but resulted in a poor alignment which could not be fine-tuned by the end-user. When installing the rotor, a dial indicator would be used to measure the eccentricity of rotation and help adjust the alignment through tightening/loosening the screws. Regarding the length of the rotor, if the ratio of the length to the gap between cylinders is in excess of 100, end effects are negligible (torque from the fluid underneath the rotor) [sec 2.4.4][6]. Due to manufacturing limitations the maximum immersion length was $70 \mathrm{~mm}$; using rotors which produced either a 1 or $3 \mathrm{~mm}$ gap the end effects could not be neglected. The bottoms of each rotor were taper into a cone (as discussed in Section 2.4.2). While it may seem reasonable to use Equation 2.128 to determine the angle $\beta$ for these rotors this relationship (Equation 2.128) was based on the assumption of a narrow gap $(\kappa \geq 0.99)$. As this criterion was not satisfied for either geometry an angle $\beta=5^{\circ}$ was used as a first order correction for the end effects. Furthermore, both cones were machined to have a truncation gap of $60 \mu \mathrm{m}$.

\subsection{Electronics}

This section explores the various electronic components utilised in the hardware and instruments developed in this thesis. An overview of which is presented as a block diagram in Figure 5.10.

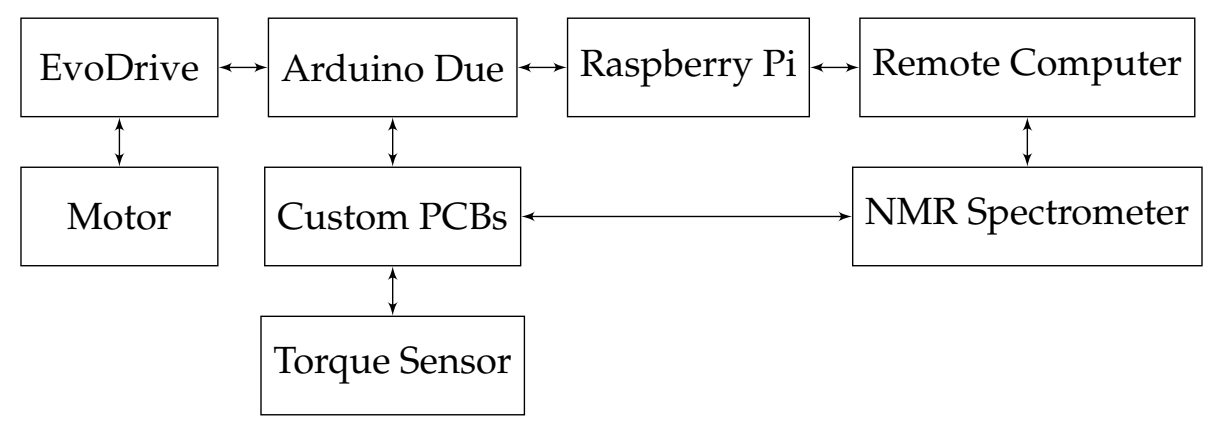

Figure 5.10: Block diagram of electronic components. 
The central component of the Rheo-NMR hardware is an Arduino Due board (Section 5.4.2). The microcontroller embedded on the Arduino Due board interprets user input to manage the motion generated by the drive system (Section 5.4.1). A user can access the Arduino Due board via a local network connection through a simple server (Section 5.4.4). Custom printed circuit boards (PCB) add additional functionality (e.g. digital interface to spectrometer and external memory) and interface with the analogue torque sensor (Section 5.4.3).

Circuit schematics, bills of material and component layouts for all custom PCBs can be found in Appendix C.

\subsubsection{Eva Robotics ST-23 and EvoDrive}

The servo-stepper motor package (ST-23) used in all of the drive-shaft units was purchased from Eva Robotics. There are three main components of the package, including: 1) stepper motor, 2) optical encoder and 3) EvoDrive controller. Specifications and application notes for the ST-23 package can be found in the product integration manual [127].

A stepper motor is a brushless DC motor specifically designed such that it moves in discrete steps. In general there are various methods in both constructing and controlling a stepper motor which define characteristics such as step size and torque output ${ }^{1}$. The motor included in the ST-23 package is a hybrid synchronous stepper motor with full steps of $1.8^{\circ}$. The windings are rated to $2.8 \mathrm{~A}$ and the motor can generate a maximum torque of $1.8 \mathrm{~N} \mathrm{~m}$. The motor is a NEMA-23 frame with a dual shaft.

When driven in either full step or half step [132] stepper motors can be used reliably in an open-loop system (provided the stepper motor has been properly rated, i.e. can produce the necessary torque for the application). Micro-stepping is a technique where the winding current is modulated as a function of time such that the motor moves in small steps between the physical poles. While this scheme provides higher position resolution (i.e. small steps) and smoother motion there is a risk that the motor may miss a step. To take advantage of micro-stepping, but maintain confidence that the motor will complete the desired motion, the Eva Robotics system utilises an optical encoder. The encoder provides a feedback loop to the EvoDrive controller so that corrections may be applied for missed steps; this closed-loop system could also be referred to as a servo motor or in the case of the stepper motor a servo-stepper motor. Practically, operating in a closed loop, the step size is limited to half the resolution of the position encoder. The optical encoder hardware installed in the ST-23 package generates 2,048 pulses per revolution which, combined with quadrature detection, is 8,192 counts per

\footnotetext{
${ }^{1}$ A general review of these concepts is outside the scope of this thesis; however, numerous descriptions of stepper motors can be found online or in textbooks from various fields, e.g. mechatronics [132, sec. 7.2].
} 
revolution. Thus the ST-23 servo-stepper motor has a reliable step size of $0.088^{\circ}$ (4096 positions per revolution) for either position or velocity commands. Additionally, Eva Robotics has implemented vector control (also referred to as field orientation control) such that the motor can be used to apply a set torque or the torque required to move the motor can be measured. Due to the resolution of this technique and variability between motors torque values are treated as a percentage equivalent to the percentage of current needed to drive the motor (e.g. $50 \%$ torque means $50 \%$ of the available drive current is flowing through the motor windings). More precise measurements can be made through calibrating a motor with known torques at similar conditions to testing.

Communication with the EvoDrive electronics is possible using a serial connection (RS232, RS485 or USB). The physical connection to the EvoDrive can be found on a custom PCB on the top of the ST-23 package. In Section 5.5.1 the software used to communicate with and control the EvoDrive will be discussed.

\subsubsection{Arduino Due and Custom Shield}

Arduino [133] is an open source platform for developing microcontroller based hardware. The wide community support and low entry costs have seen a rapid rise in the popularity of Arduino in hobbyists and researchers alike [134]. In this thesis an Arduino Due [135] was used to manage interactions between users, the NMR spectrometer, the torque sensor and other peripheral devices. At the heart of Arduino Due board is a 32 bit ARM Cortex-M3 processor (AT91SAM3X8E, Atmel Corporation [136]). The processor was programmed with the Arduino IDE via the native high speed USB port on the SAM3X.

To utilise the features of the SAM3X processor a custom printed circuit board (PCB) was designed and constructed. This two layer PCB was designed to mount directly onto the Arduino Due; this style of board is typically referred to as a "shield" in the Arduino community. During experiments the Arduino Due and custom PCB (shield) are mounted to the ST-23 servo-stepper motor package (Figure 5.11a).

The shield included the physical connection for the serial interface with the EvoDrive via a logic level conversion circuit (the EvoDrive electronics operated at $5 \mathrm{~V}$ while the SAM3X operated at $3.3 \mathrm{~V}$ ). Additionally, SMB style coaxial connections were available to interface the drive-shaft unit with the Bruker spectrometer (Figure 5.11b); as the spectrometer digital lines operated at $5 \mathrm{~V}$ the input channels were stepped down via a voltage divider and the output channels were driven from a $5 \mathrm{~V}$ power supply via transistors.

A micro-SD card socket and media on the shield provide removable external memory. The processor and micro-SD card communicate using the serial peripheral interface (SPI) bus. The primary use of the micro-SD card is for logging data during experiments 


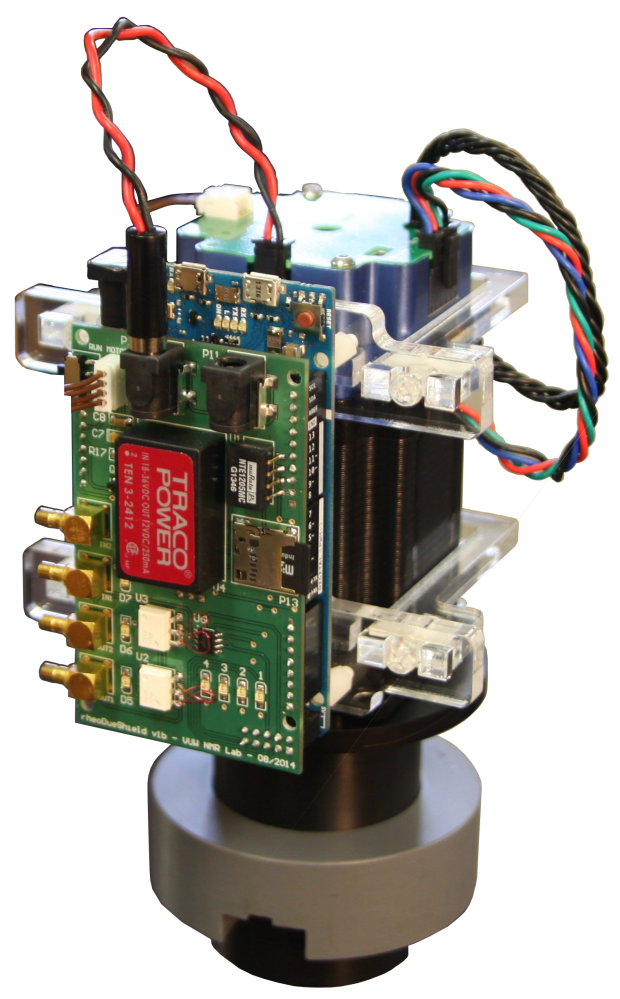

(a)

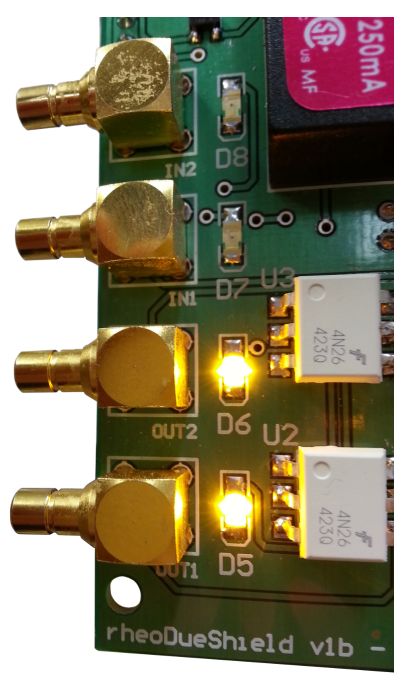

(b)

Figure 5.11: (a) Arduino Due development board and custom shield mounted to the ST-23 package for NMR experiments. (b) Digital lines for real-time synchonisation of motion and NMR pulse sequence. Note: The shield shown here is an earlier version and does not match the schematics/PCB layout found in Appendix C.

(e.g. frequency, input/output states, torque); however, it also contains a settings file. Utilising this external memory these settings are non-volatile and read on boot to prevent the electronics from reverting to the default settings in the firmware; the settings can be updated by the user via serial command or user interface.

The SAM3X processor also has internal quadrature decoding which can be used with the optical encoder in the ST-23 package (Section 5.4.1) to monitor the frequency of rotation without having to poll the EvoDrive. The shield has a physical connection to tap into the encoder signal without affecting the normal operation of the ST-23 feedback loop. While this functionality has been tested, currently the firmware (Section 5.5.1) does not utilise this feature.

To help when assembling the drive-shaft unit on the bench a hand-held push-button pendant could be plugged into the shield. When the push-button was depressed, the motor would rotate at a pre-defined frequency (adjustable through user interface, Section 5.5.2) 
The Arduino Due board and shield were powered from the same $24 \mathrm{~V}$ supply used by the ST-23 package with various voltages provided by components on the shield.

\subsubsection{Torque Interface Card and Custom Electronics}

The Vibrac torque sensor (Section 5.3.3) required a signal conditioning card (e.g. 1312 Interface Card, Vibrac [137]). The card required $\pm 15 \mathrm{~V}$ to drive the board and transducer; additionally, an input DC voltage was used to zero the torque signal. The board had two analogue outputs (torque and reference channels) were the $\pm 10 \mathrm{~V}$ torque signal was proportional to the measured torque with $10 \mathrm{~V}$ representing the full range torque value. The polarity of the torque signal indicated the direction of the torque (i.e. $\mathrm{CW}$ or CCW).

An Arduino Due (Section 5.4.2) was used with the torque sensor drive-shaft variant, however, because the additional electronic components could not fit onto a single PCB (e.g. shield) three stackable PCBs were designed and assembled. These boards had the basic components as described in the previous section (e.g. serial communication, micro-SD card, digital input/output channels) in addition to $\pm 15 \mathrm{~V}$ power supplies, an analogue-to-digital converter (ADC) and a digital-to-analogue converter (DAC). The Arduino Due and stacked PCBs were mounted with the interface card onto a single piece of acrylic (Figure 5.12) for easy set-up on the bench or above the NMR magnet.

The external 12 bit DAC was controlled by the SAM3X via SPI; a reference voltage for the DAC was provided by an external chip. The DAC was used to generate the $-10-$ $0 \mathrm{~V}$ zeroing signal. The SAM3X processor includes an internal 12 bit DAC, however, the output range (nominally $0-3.3 \mathrm{~V}$ ) was not sufficient for the zeroing signal. Additional components could have been used to invert and add gain to this output range, however, the external device was sufficient for the work in this thesis.

A standalone two channel 12 bit ADC was used to digitise the analogue torque and reference signals. The chip that was used could accept a true bipolar analogue signal between $\pm 10 \mathrm{~V}$ and was ideal for use with the interface card. The SAM3X also includes an internal $12 \mathrm{ADC}$, however, as was the case for the DAC (described above), additional components would have been necessary to connect the $\pm 10 \mathrm{~V}$ signal safely to the processor.

\subsubsection{Raspberry Pi Server}

To avoid driver issues when connecting the Arduino Due board to various operating systems a Raspberry Pi 1 Model B+ single-board computer was used as a bridge between a local network and the USB connection of the control electronics. The Pi runs the Raspian (Debian, Linux) operating system and uses the relay utility Socat 


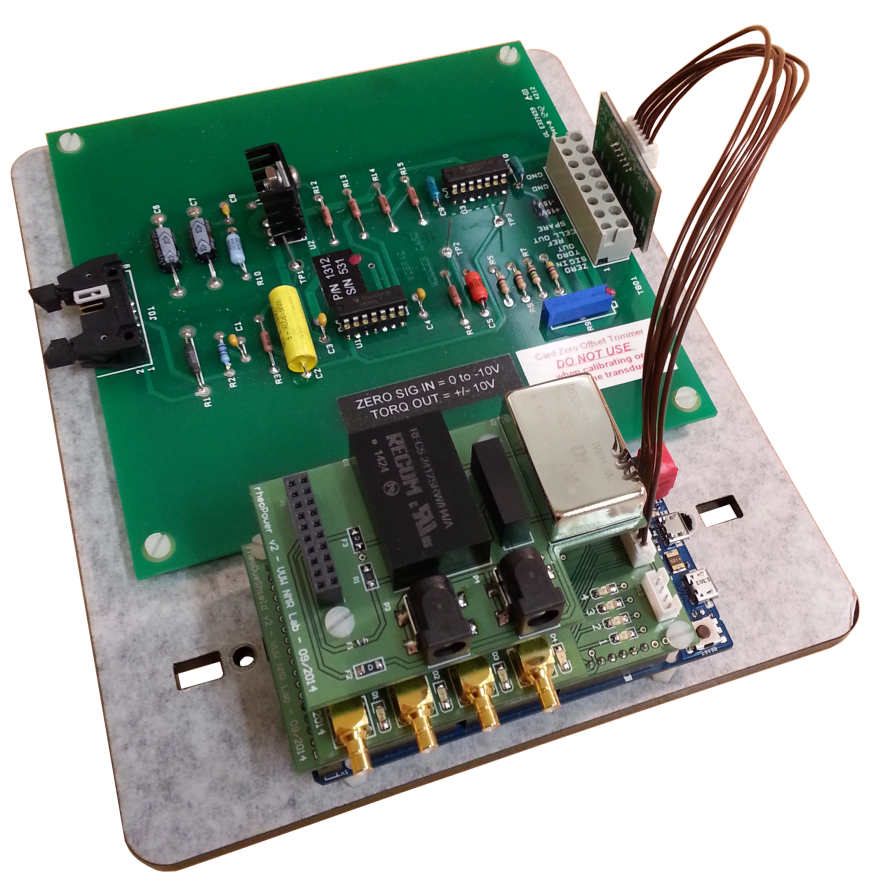

Figure 5.12: Vibrac torque interface board (back) shown connected to the Arduino Due and custom printed circuit boards (front).

(v1.7.2.3) [138] for bi-directional data transfers between a TCP port (network connection) and a TTY device (USB connection).

Additionally, the Raspberry Pi computer can run the graphical user interface (described below in Section 5.5.2). Utilising a secure shell (SSH) network connection with X11 (mechanism for forwarding graphical interfaces) the control software can be run from a remote machine regardless of the remote computer's operating system. This functionality simplifies implementation by eliminating: 1) the need to write a crossplatform control programme and 2) the maintenance of multiple software installations.

\subsection{Software}

The instrument developed in this thesis relied on two types of software $2: 1$ ) the Arduino Due firmware and 2) the user interface.

\footnotetext{
${ }^{2} \mathrm{~A}$ Git repository with all software is available at http://oscar.ecs.vuw.ac.nz/ tb/ motioncontrol/.
} 


\subsubsection{Firmware}

The Arduino Due board interfaces with the ST-23 through a UART device communicating using the RS485 standard [128]. An Arduino library [139] was developed by Eva Robotics for an 8 bit microcontroller (including the EVA Robotics EvoDuino) to interface with the EvoDrive. This library was modified for use with the 32 bit SAM3X core of the Arduino Due. Utilising the modified EvoDuino library (referred to as EvoDuino4Due) an embedded controller (Section 5.4.2) was developed and programmed using the Arduino IDE. The firmware ${ }^{3}$ for the Arduino Due board was written in $\mathrm{C} / \mathrm{C}++$ and compiled to machine code through the default Arduino build process. This firmware serves as an abstraction layer between the user and the ST-23 package to convert rheology experimental parameters into the EvoDrive motion commands (e.g. setVelocity, setTorque). The two major tasks of the firmware are: 1) managing the serial communication between the Arduino board and a host system (i.e. desktop computer on local network) and 2) real-time control of the EvoDrive.

The firmware receives commands using a string of 32 ASCII characters. A review of the serial command structure (options and key string formatting) can be found in Appendix D.

\section{Motion Routines}

A full list of available motion profiles can be found in Appendix D. In the section below a brief introduction is given to oscillatory and start-up follow routines.

Using this system, oscillatory shear profiles are available for Rheo-NMR experiments. Although the initial motivation was to develop motion routines for non-linear viscoelasticity rheology (i.e. large amplitude oscillatory rheology, LAOS) the method can also be used for small angle oscillatory (linear viscoelasticity) rheology studies. When the firmware receives an oscillatory motion command a position vector is calculated for one period of the sinusoidal motion based on the desired strain amplitude and angular frequency. Once the motion begins, at regular intervals, the firmware uses the current shaft position to calculate a velocity command so that over the following interval the shaft (in theory) would reach the next coordinate in the position vector. In this way the programme is constantly self-correcting for small errors in motion. The number of points within the position vector is based upon the period of motion and the position update frequency ( $50 \mathrm{~Hz}$ by default). A timer interrupt is used to trigger the update of motion.

To investigate the response of fluids during acceleration from a steady state (e.g stationary or constant velocity) a start-up profile routine has been developed. Previously,

\footnotetext{
${ }^{3}$ see footnote 2; the firmware source code is included in this repository and can be viewed online [140].
} 
start-up flow could be studied by ramping the motor frequency in discrete steps, however, this new approach takes advantage of the acceleration setting of the EvoDrive. Based upon a desired maximum shear rate and a desired interval of time the firmware calculates an acceleration value and updates the motor settings. The start of this motion programme can be triggered by the NMR spectrometer to synchronise the motion profile and NMR acquisition. Additionally, a delay is available between the start of the motion and the set acceleration command which allows NMR data to be collected in a reference state prior to deformation. After the maximum shear rate is reached the motion will be stopped; alternatively, this routine could be continuously looped in a sawtooth type pattern.

\section{Motor Settings}

In addition to real-time management of the motion the firmware also manages the read/ write functions of the micro-SD card, interfaces via digital I/O with the Bruker spectrometer and controls both the analogue-digital converter (ADC) and digital-analogue converter (DAC) used as part of the analogue torque measurement system.

If desired the firmware can write motion parameters, torque measurements and input/output state to the mounted micro-SD card. Functions are available to create and delete files and also transfer data to a remote system via the USB connection. As part of the datalogging routine, the firmware can also stream values directly to the USB connection (which can be used for real-time monitoring on a remote system).

The firmware has a number of routines related to the torque measurement system (including debug functions for the ADC and DAC) the most important of which is a successive approximation routine to zero the torque sensor.

\subsubsection{User Interface}

An additional layer of software abstraction is provided by a series of user interfaces which allow users to set-up experiments in terms of rheological parameters (e.g. shear rate, shear stress) but sends commands to the motor system in generic motion terms (e.g. angular frequency, torque). Two types of UI were implemented including a standalone control programme and modules for the TopSpin environment.

\section{Stand-alone GUI}

For debugging, stand-alone operation (i.e. bench-top rheometer) and advanced control a graphical user interface (Figure 5.13) is available for the various new Rheo-NMR instruments. The module is written in Python (v2.7) [141] and utilises the cross-platform 
application framework Qt (via the Python-Qt wrapper, Pyside v1.2 [142, 143]) for visualisation.

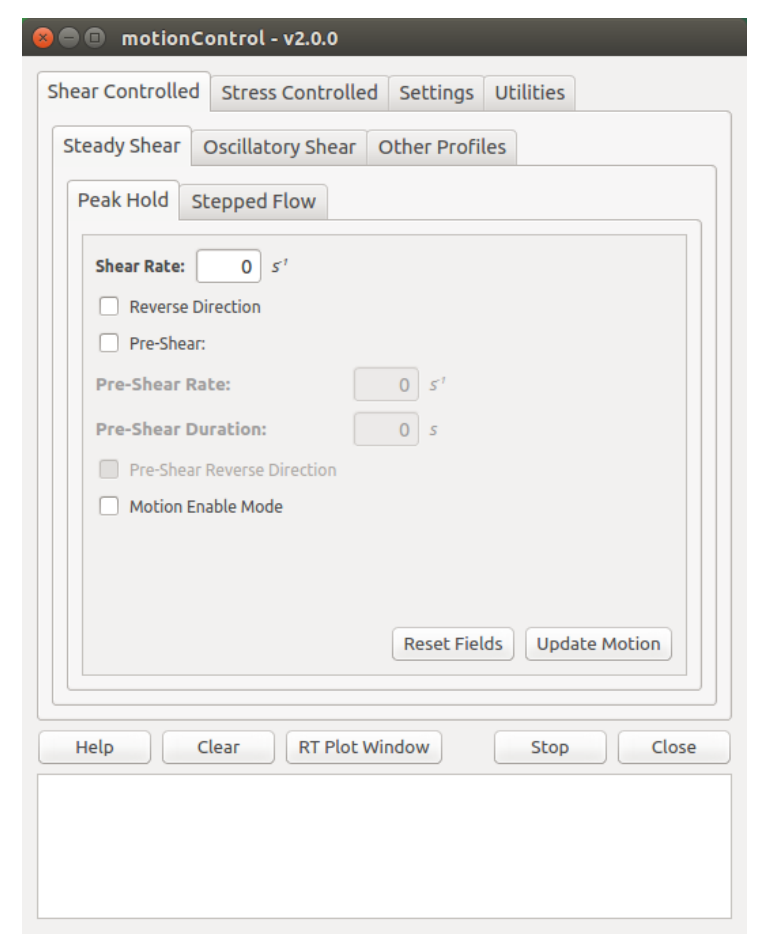

Figure 5.13: motionControl graphical user interface.

The graphical user interface (GUI) is called "motionControl.py" and in addition to setting up experiments manages data transfers from the micro SD card, provides a simple interface for viewing and updating motor settings and can be used for various debugging tasks (e.g. measure ADC channel, toggle outputs, read state of inputs).

Set-up for experiments is partitioned into various functions (modelled after the TA Instruments control software). Both strain controlled or stress controlled experiments are available. Additionally, the user can define the type and dimensions of the shear geometry.

\section{TopSpin Modules}

While the torque sensor drive-shaft variant can be used as a stand-alone instrument the primary purpose of the hardware is to be used with Bruker NMR systems. For ease of operation, programmes have been written for the Jython environment within the Bruker NMR control software suite, TopSpin (tested on TopSpin v2.1, v3.1PV and v3.2). Python scripts run within TopSpin can be used to simultaneously set up the parameters for the NMR acquisition and communicate with the motor electronics. Furthermore, higher level modules have been written so that multiple NMR experiments (with mo- 
tor control) can run sequentially without user input (see Figure 5.14). This would be analogous to setting up a stepped flow (or similar) experiment on a rheometer where sample properties are measured at multiple shear rates.

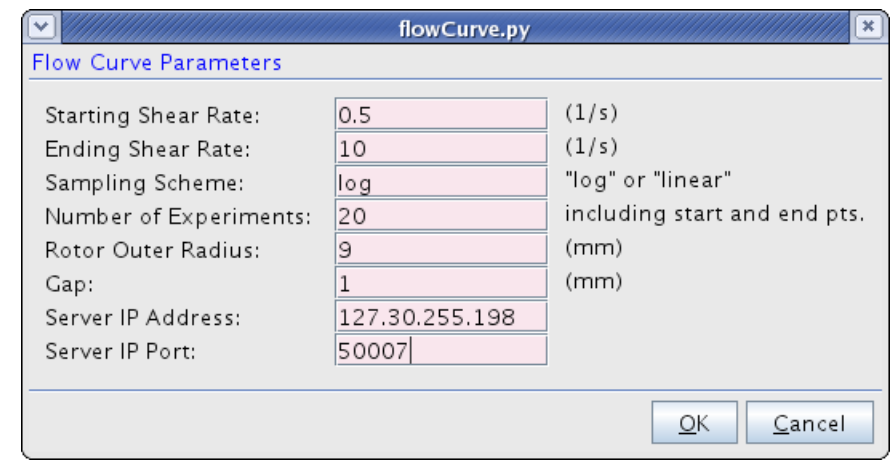

Figure 5.14: TopSpin interface module.

For real-time synchonisation of the NMR pulse sequence and drive-shaft motion digital lines (Figure 5.11b) are available to trigger either the NMR spectrometer or motor. Bruker spectrometer consoles (tested on Avance and Avance III) have a range of TTL-like outputs which can be used to trigger the motor. This function is particularly important for experiments investigating start-up flow. Additionally, Bruker consoles have a limited $(<4)$ amount of trigger lines which allow the pulse sequence to be controlled by motion parameters (e.g. a certain position or speed). This function is particularly useful for periodic experiment (e.g. oscillatory shear) where the NMR acquisition should be executed at a precise position of the drive-shaft.

\subsection{Conclusion}

Various pieces of hardware were presented in this chapter and each individually contribute to the goals laid out in the introduction: 1) improving shear geometries, 2) new experimental protocols and 3) simultaneous bulk rheology. Assembling these components into complete instruments there are three main assemblies that will be discussed in the following chapters: 1) drive-shaft unit with integrated torque sensor, 2) wide bore Rheo-NMR kit compatible with the Bruker variable temperature control, offering a range of shear geometries and numerous never before realised experimental protocols (e.g. steady shear, oscillatory strain, stepped functions and spectrometer interface) and 3) a super wide bore instrument including all the features described for the wide bore design. 


\section{Chapter 6}

\section{Hardware Validation Studies}

\subsection{Introduction}

This chapter explores the hardware introduced in Chapter 5. Moving forward from the designs previously described, proof of concept and validation studies will now be presented.

\subsection{Shear Geometries}

This section explores the new concentric cylinder and planar-cylindrical hybrid shear geometries. In both cases water (Newtonian behaviour) was used in combination with $1 \mathrm{D}$ and 2D velocity imaging pulse sequences to compare the measured flow to theory.

\subsubsection{Concentric Cylinder (Super Wide Bore)}

The $1 \mathrm{D}$ velocity imaging pulse sequence used to validate the super wide bore concentric cylinder shear geometries can be seen in Figure 6.1a. This pulse programme utilised double slice selection (Section 3.6.1) by exciting slices along the $y$ (velocity direction) and $z$ (vorticity direction) axes. A graphical description of this sampling scheme can be seen in Figure $6.1 \mathrm{~b}$ where the 1D imaging was along the $x$ axis (velocity gradient direction) and displacements were encoded in the $y$ (velocity) direction.

Velocity profiles of water were measured in five concentric cylinder geometries. The curvature ratio $\kappa$ (Equation 5.1) for three of these geometries was the same $\kappa=0.89$ with dimensions $\frac{r_{\mathrm{i}}}{r_{\mathrm{o}}}=\frac{8.00}{9.00}, \frac{11.10}{12.50}$ and $\frac{15.10}{17.00}$. The remaining cells had either a larger (0.96) or smaller $(0.71) \kappa$, respectively indicating a smaller or larger difference in curvature.

Experiments were conducted across a reasonable range of shear rates $\left(1-10 \mathrm{~s}^{-1}\right.$ shown) with the experimental data compared to the theoretical velocity profile (Equation 2.117). Examples of these comparisons for the three shear geometries with the 


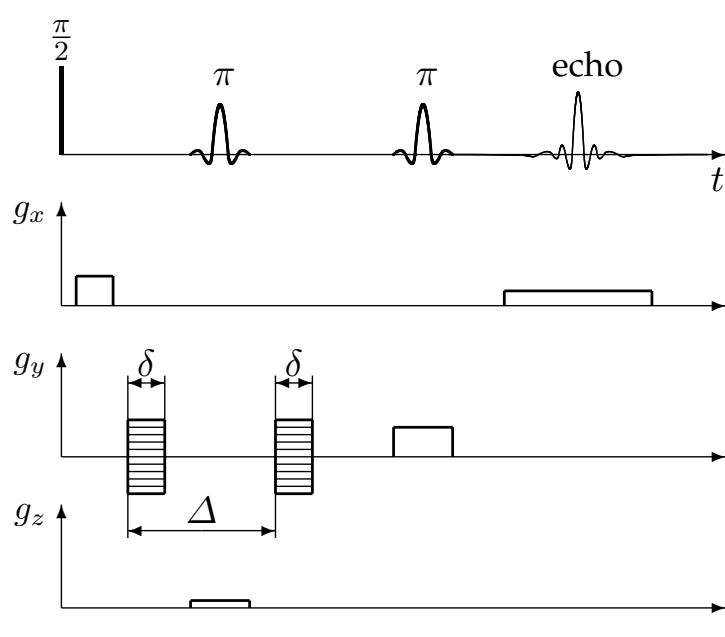

(a)
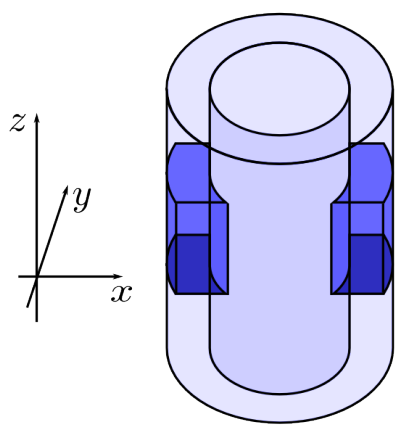

(b)

Figure 6.1: (a) Pulse program diagram for Pulsed Gradient Spin Echo (PGSE) motion encoding with double slice selection and 1D imaging acquisition. (b) Illustration of the imaging region. In this study, the $x$ axis aligns with gradient direction, the $y$ axis aligns with velocity direction and the $z$ axis aligns with vorticity direction.

same $\kappa$ can be seen in the top panel of Figure 6.2 and comparisons for the remaining two geometries can be found in the middle panel of Figure 6.2. In both panels, for each geometry, a velocity profile is shown which is the average of the two gaps on either side of the rotor. Additionally, the gap position and velocity values were both normalised to simplify comparisons between different geometries.

For the geometries with the same curvature (top panel Figure 6.2), all normalised example datasets agreed and also fit the shape of the theoretical curve. However, a stark difference in the velocity profile can be observed between the two geometries with differing $\kappa$ values (middle panel Figure 6.2), although the normalised measurements again agreed with the individual theoretical predictions.

In the top panel of Figure 6.2 the velocity gradient is nearly linear while curvature is apparent in the $\kappa=0.71$ profile (middle panel) due to the curvature difference between the two boundaries. To demonstrate the accuracy of the NMR technique and the functionality of the hardware the bottom panel of Figure 6.2 plots the coefficent of determination $\left(R^{2}\right)$ from fitting the experimental data to theory as a function applied shear rate (for simplicity the applied shear rate is defined as in Equation 2.122, where curvature effects are neglected) for the various shear devices. As shown all are close to $R^{2}=1$. 

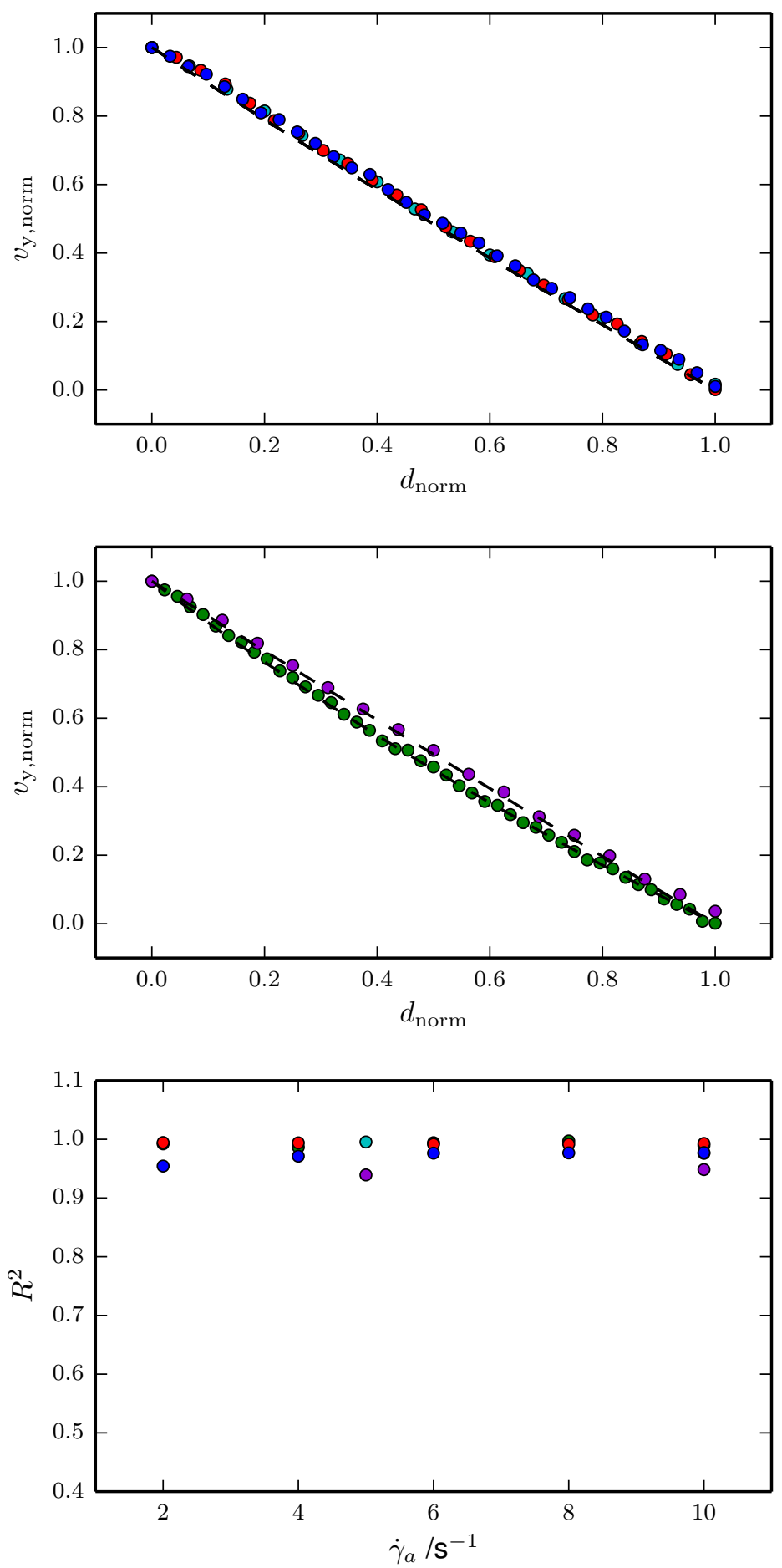

Figure 6.2: Normalised velocity measurements for three shear cells with the same stress variation across the gap (top); $\kappa=0.89, \frac{r_{\mathrm{i}}}{r_{\mathrm{o}}}=\frac{8.00}{9.00}(\diamond), \frac{11.10}{12.50}(\bullet), \frac{15.10}{17.00}(\bullet)$. And the two geometries with the greatest difference in stress variation across the gap (middle); $\kappa=0.71$ (॰) \& 0.96 (॰). The dashed line represents the theoretical velocity profile. $R^{2}$ values from comparing velocity profiles to the theoretical curve as a function of applied shear rate (bottom). 


\subsubsection{Planar-Cylindrical Hybrid}

Velocity measurements were conducted on the planar-cylindrical hybrid device using a 2D PGSE-RARE sequence [80,144]. Water doped with $0.15 \mathrm{mM} \mathrm{GdCl}_{3}\left(T_{1}=0.46 \mathrm{~s}\right.$, $T_{2}=0.23 \mathrm{~s}$ ) was used as the calibration fluid. The loaded hybrid device was placed in the RF coil at an arbitrary orientation. For these experiments, the PTFE spacer described at the end of Section 5.2.3 was not used. During experimental set up the axes of the inplane gradients were rotated to align the $x$ parallel to the long axis of the geometry and the $y$ axis parallel to the short axis (Figure 6.3). The field of view of the image was $25 \mathrm{~mm}(x)$ by $12 \mathrm{~mm}(y)$ with 128 pixels collected along each axis; the signal was averaged over a $3 \mathrm{~mm}$ slice in the $z$ direction.

The Reynolds number $R e$ for planar Couette flow can be determined with [125]

$$
R e=\frac{v d}{4 \nu}
$$

where $v$ is the velocity of the belt, $d$ is half the distance across the fluid domain and $\nu$ is the kinematic viscosity. Previous flow measurements conducted with 3D particle tracking velocimetry reported a laminar flow regime for $R e<320$ [125]. For the hybrid geometry constructed in this thesis the distance between band and stationary wall was $1 \mathrm{~mm}(d=0.5 \mathrm{~mm})$. Using water at room temperature as the test fluid $\left(\nu \approx 1 \mathrm{~mm}^{2} \mathrm{~s}^{-1}\right)$ and shear rates $\dot{\gamma}=\frac{v}{2 d}$ between $1-20 \mathrm{~s}^{-1}$ laminar flow was expected.

In Figure 6.3 the normalised results of the 2D PGSE-RARE experiment are compared to finite element analysis simulations. Simulations were made with the computation fluid dynamics module of the COMSOL Multiphysics software [145] (v4.3); the dimensions within the COMSOL model matched the construction of the hybrid device, the physical properties of water were used to describe the fluid and laminar flow was assumed.

Qualitatively, the comparison of the NMR experiments and numerical simulations (Figure 6.3) is quite exciting. However, to evaluate the utility of the hybrid device for generating simple shear deformation the velocities measured by NMR were further compared to theory. In Figure 6.4, the velocity profile between the moving band and stationary wall has been plotted. The normalised data (marks) reported in Figure 6.4 were extracted from the centre pixels (approximately $x=0 \mathrm{~mm}$ ) of the $2 \mathrm{D}$ velocity image (flow encoded in $x$ direction). The slope of the straight line (solid) has a magnitude equal to unity. The data appear to have a linear relationship and suggests that continuous simple shear deformation is possible within a wide bore superconducting magnet. 

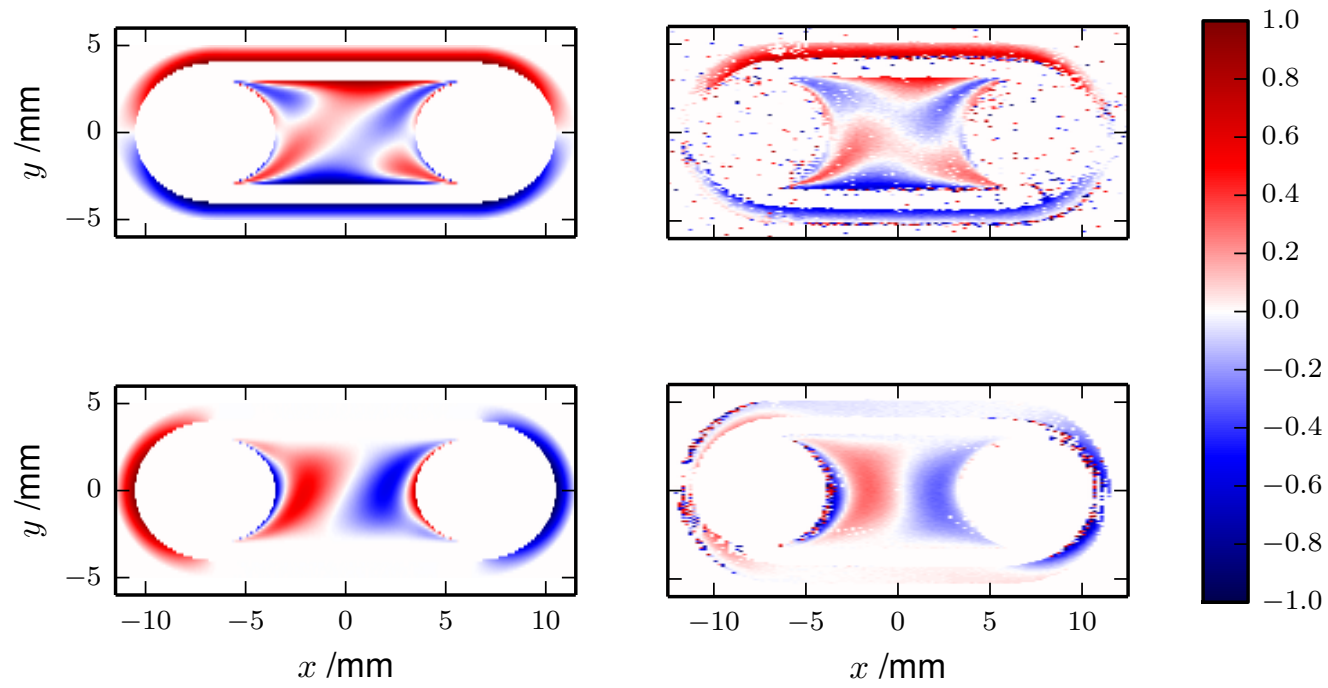

.2
.6
.8
.0

Figure 6.3: Comparision of 2D COMSOL simulation results (left column) to 2D NMR velocity images (right column). The top row compares the $x$ component of velocity while the bottom row compares the $y$ component of velocity. Note: The PTFE spacer described at the end of Section 5.2.3 was not used during this experiment.

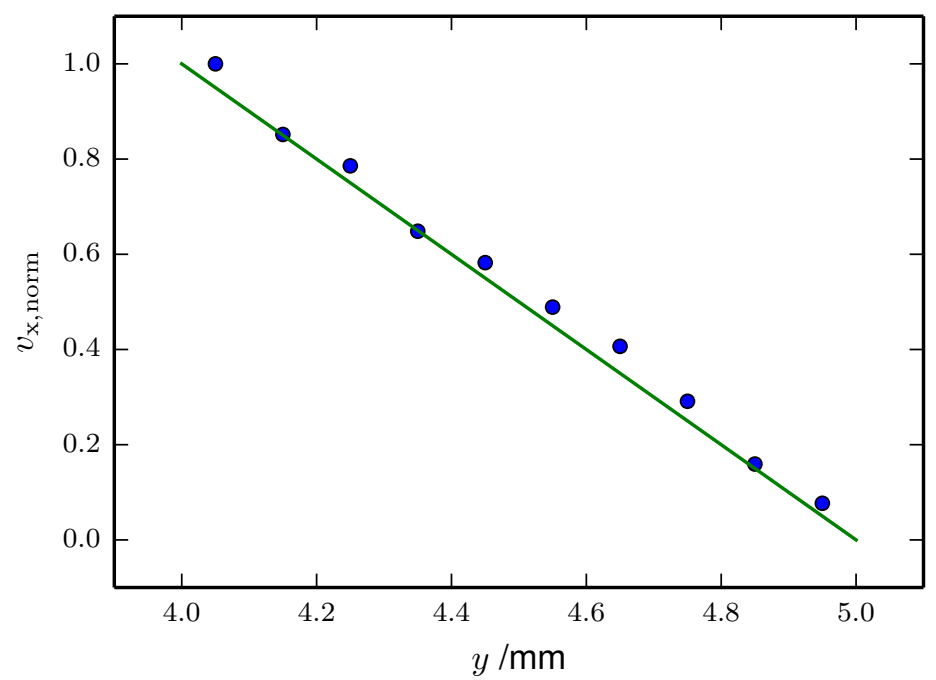

Figure 6.4: Velocity profile for single gap (extracted from 2D NMR measurement at $x=0$ ) compared to straight line (theoretical model for simple shear). 


\subsection{Drive-Shaft (Generic)}

In this section the functionality of the drive-shaft units will be explored. The performance of the shear profiles (Section 6.3.1) and the digital interface between the motor and NMR spectrometer (Section 6.3.2) are applicable to all iterations of the new driveshaft units (wide bore, super wide bore and torque sensor variants). With regard to simultaneous bulk rheology measurements (e.g. torque) exciting results will be presented from estimating torque from the servo-stepper motor where an auxiliary transducer is not required (Section 6.3.3). Proof of concept work for the drive-shaft unit with the inline analogue torque transducer will be discussed in the following section (6.4).

\subsubsection{Strain Rate Controlled Motion Profiles}

In Section 5.5.1 various motion profiles were introduced; Figure 6.5 shows representative data for oscillatory, controlled start-up and shear quench ${ }^{1}$ experiments. For the oscillatory and start-up profiles the motor data (either angular position $\phi$ or frequency f) logged as a function of time behaved as expected.

With regard to oscillatory motion it is currently possible to access angular frequencies up to $20 \mathrm{rad} \mathrm{s}^{-1}$ for strain amplitudes up to 1 (for the concentric cylinder with $r_{\mathrm{i}}=8 \mathrm{~mm}$ and $r_{\mathrm{o}}=9 \mathrm{~mm}$ ). The strain amplitude can be further increased for lower values of angular frequency; additionally, utilising concentric cylinders with varying dimensions could also expand this parameter space.

The bottom panel of Figure 6.5 depicts a shear quench profile where the dataset was included to illustrate one feature of continuous motion with the EvoDrive. Specifically, at high shear rates (high frequencies), oscillations are evident; however, at the lower rotation speed the magnitude of this feature is reduced. This ripple is caused by the closed-loop feedback of the EvoDrive system and can be fine-tuned by changing the steps per revolution parameter setting on the motor. As previously described the EvoDrive system drives the stepper motor via micro-stepping (Section 5.4.1); the steps per revolution parameter is used to change the resolution of the micro-stepping routine. At low frequencies $(<0.1 \mathrm{~Hz})$ a high steps per revolution parameter is crucial for smooth continuous motion; however, for high frequencies this parameter can be reduced without any loss of performance.

\footnotetext{
${ }^{1}$ Shear quench (or pre-shear), involves preconditioning a sample at a higher shear rate for a period prior to making a rheological measurement at some lower shear rate; typically this method is used to establish the same shear history between multiple tests.
} 

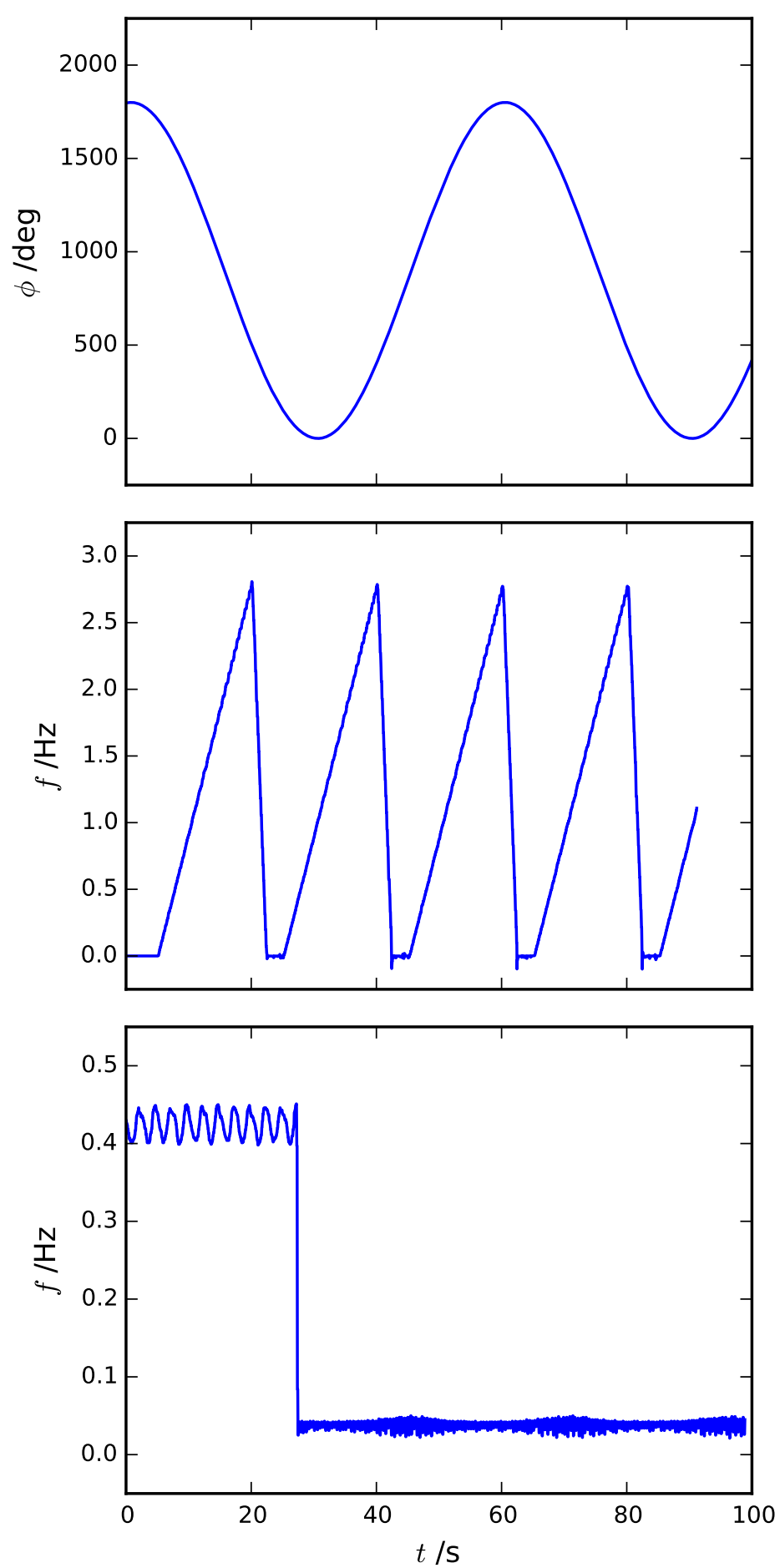

Figure 6.5: Oscillatory shear waveform with angular position as a function of time (top); "Sawtooth" profile with motor frequency plotted as a function of time (middle); and example of stepped strain with motor frequency plotted as a function of time (bottom). 


\subsubsection{NMR Acquisition Triggered by Motor}

To demonstrate the functionality of the digital interface between the drive system and the NMR spectrometer a 1D velocity image was acquired during an oscillatory shear profile. This experiment utilised the NMR pulse programme illustrated in Figure 6.6. With regard to the NMR excitation and acquisition the sequence is the same as that used for the super wide bore experiment (Section 6.2.1).

In preparing the experiment a motion command was sent to the drive system via the serial (USB) connection. However, the motor did not start moving until a positive edge was seen on the TTL output channel of the spectrometer, identified as the motion enable line in Figure 6.6.

Once the experiment was started and the motion began each excitation was triggered by the drive electronics using a trigger input on the Bruker spectrometer. As part of the serial command the drive system was told how many triggers to generate per period (details on the oscillatory motion command can be found in Section D.1.3). In the TopSpin pulse sequence there was a conditional statement line before the initial hard $90^{\circ}$ pulse which waited to see a positive edge on the trigger channel. This is depicted in Figure 6.6 where the start of the $90^{\circ}$ excitation is timed with the low to high transition of the trigger line.

To help illustrate the data acquisition Figure 6.7 shows the timing of the triggering events on the applied oscillatory shear profile. In this experiment 32 trigger pulses were sent during one period of the sinusoidal motion; however, data was collected for two complete periods. A shear amplitude of 100 and an angular frequency of $0.1 \mathrm{rad} \mathrm{s}^{-1}$ was used resulting in a period of approximately $63 \mathrm{~s}$ and about $2 \mathrm{~s}$ between each NMR acquisition.

The sample fluid was distilled water. The geometry used for this experiment included a rotor with $r_{\mathrm{i}}=8 \mathrm{~mm}$ and a cup with $r_{\mathrm{i}}=9 \mathrm{~mm}$; the rotor included a $10 \mathrm{~mm}$ diameter void at its centre which was also loaded with the distilled water sample. The measured signal for each trigger was averaged through eight successive scans for each of the four $q$-gradient strengths. Capturing motion over two periods the total experiment time was approximately $67 \mathrm{~min}$. The processed data is shown in the left plane of Figure 6.8. The dataset is represented in two dimensions constructed from $1 \mathrm{D}$ velocity profiles oriented along the horizontal axis and position along the oscillatory waveform indicated by the vertical axis (i.e. position of the respective trigger). To illustrate this the right plane of Figure 6.8 shows the applied sinusoidal profile (solid line) and discrete positions of the individual triggers (markers). Each 1D velocity profile was constructed from successive averages and PGSE gradient strengths and is thus a time average over the entire experiment. Despite the long experiment time ( $>60 \mathrm{~min})$ the coherent velocity profiles suggest that the trigger scheme between the motor electronics and NMR 


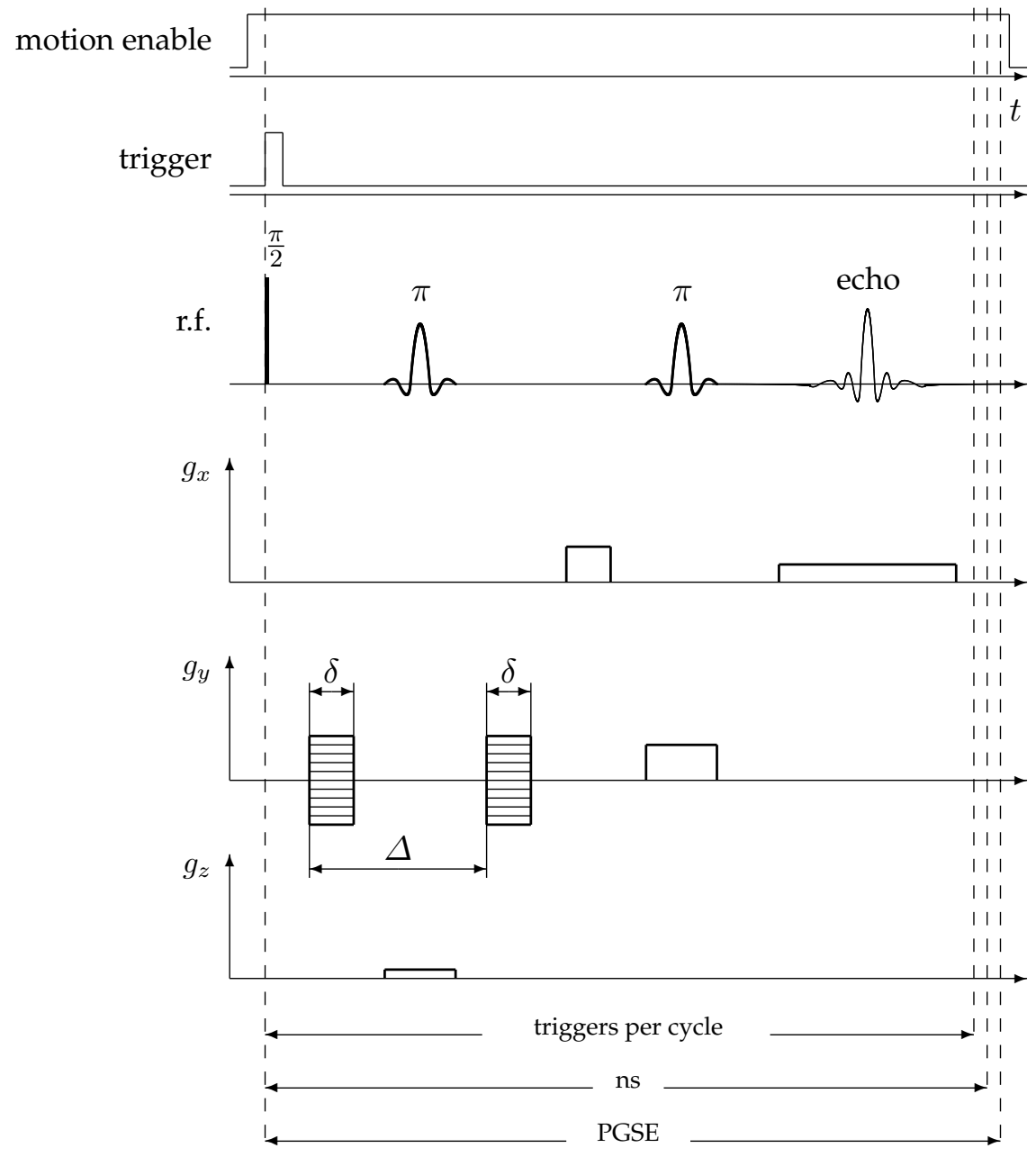

Figure 6.6: Pulse programme used for oscillatory 1D velocity imaging. 


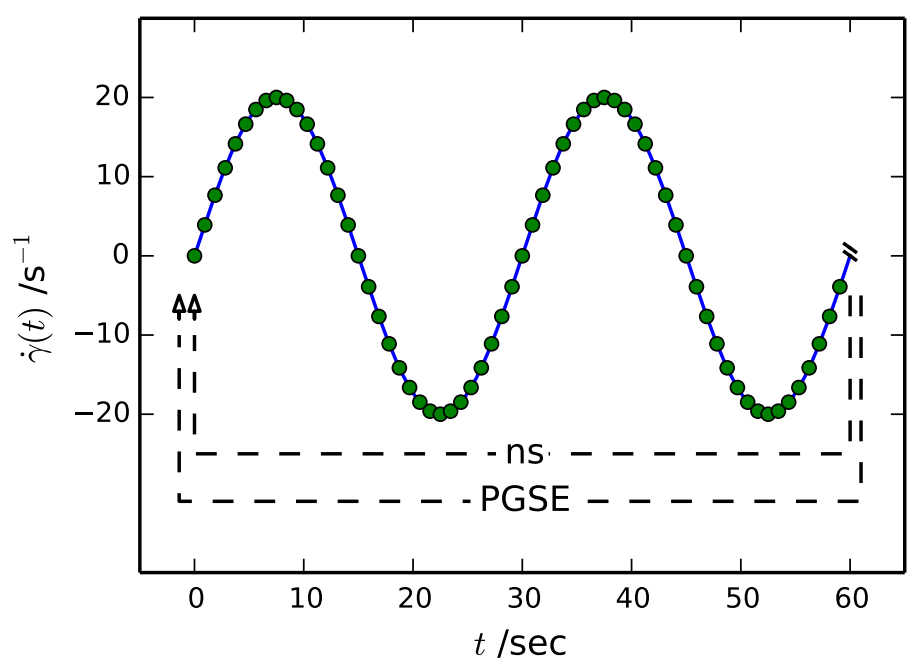

Figure 6.7: Pictorial representation of the NMR acquisition based on an oscillatory shear profile.
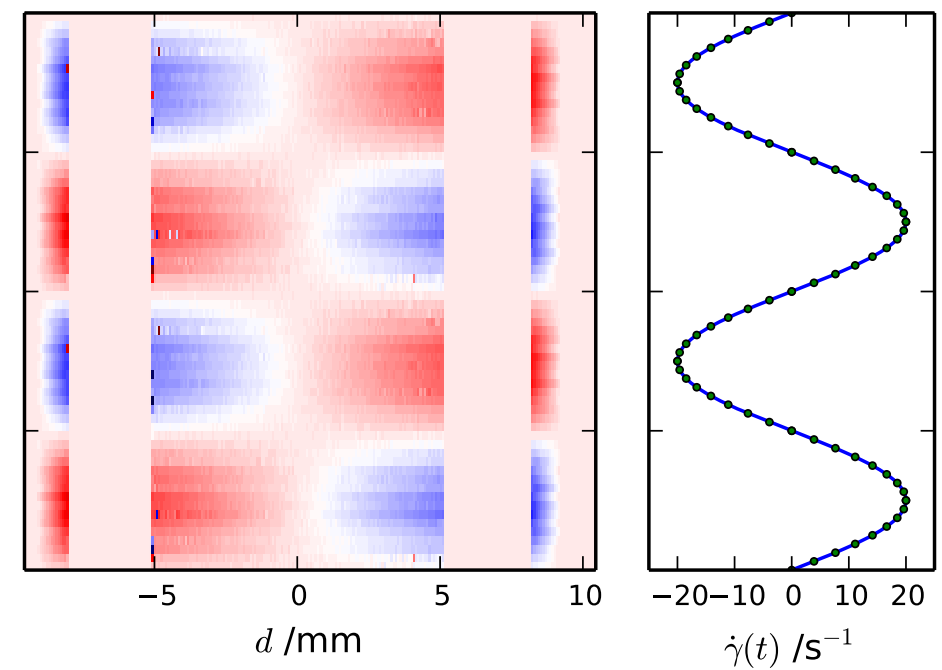

Figure 6.8: 1D velocity profiles acquired using triggers based on the position of the motor shaft. To the right of the velocity map is an illustration of the applied sinusoidal waveform. 
spectrometer worked as expected. Furthermore, it can be inferred that the oscillatory motion was stable over the length of the NMR experiment.

\subsubsection{Torque Measurements (Vector Control)}

Previously it was mentioned that the EvoDrive can utilise a vector control method to either generate a constant torque output or measure the torque required to generate the desired motion (Section 5.4.1). When reporting a measured torque value the EvoDrive system returns a percentage of the maximum torque. This maximum torque value varies between motors due to both the stated torque per current rating and differences in construction. Currently, the drive-shaft units developed for this thesis utilise a stepper motor capable of producing a torque of $1.8 \mathrm{Nm}$ at $2.8 \mathrm{~A}$. Using the EvoDrive settings it is possible to limit the maximum torque by changing the amount of current available to the motor, which can be updated via the serial interface. From the returned percentage $M_{\%}$, a torque $M$ can be calculated

$$
M=\left(\frac{M_{\%}}{100}\right)\left(\frac{M_{\text {motor }}}{I_{\text {motor }}}\right) I_{\max }
$$

where $M_{\text {motor }}$ and $I_{\text {motor }}$ come from the motor rating and $I_{\max }$ is the EvoDrive current setting. In practice individual motors slightly deviate from the listed nominal torque and current rating requiring calibration under a known torque.

To test the possibility of making rheological measurements with the ST-23 system a silicone oil with a kinetic viscosity of 100k cSt (PN 378437, Sigma-Aldrich; viscosity was verified with a commercial rheometer) was loaded into the concentric cylinder shear cell with $r_{\mathrm{i}}=8 \mathrm{~mm}$ and $r_{\mathrm{o}}=9 \mathrm{~mm}$. To simulate different fluid viscosities, experiments were conducted at three fill heights (i.e. 45, 71 and $100 \mathrm{~mm}$ ). For each of these samples the torque required to move the rotor was recorded as a function of applied shear rate over $1-30 \mathrm{~s}^{-1}$ (top panel of Figure 6.9).

The sample with a fill height of $100 \mathrm{~mm}$ was used as a reference standard; using Equations 6.2 and 2.109 (ignoring end effects) the percent torque measurements were converted to a torque and then shear stress. The slope from a linear fit (with intercept set to zero) was used to estimate the viscosity. From the calculated viscosity and the known viscosity (determined using a commercial rheometer) a calibration factor was generated.

The raw measurements in the two remaining datasets (fill heights 45 and $71 \mathrm{~mm}$ ) were similarly converted into shear stress values before the calibration factor (determined above) was applied to each point. The corrected flow curves for these two datasets can be seen in the bottom panel of Figure 6.9. The horizontal error bars represent the standard deviation of the applied shear rate while the vertical error bars 

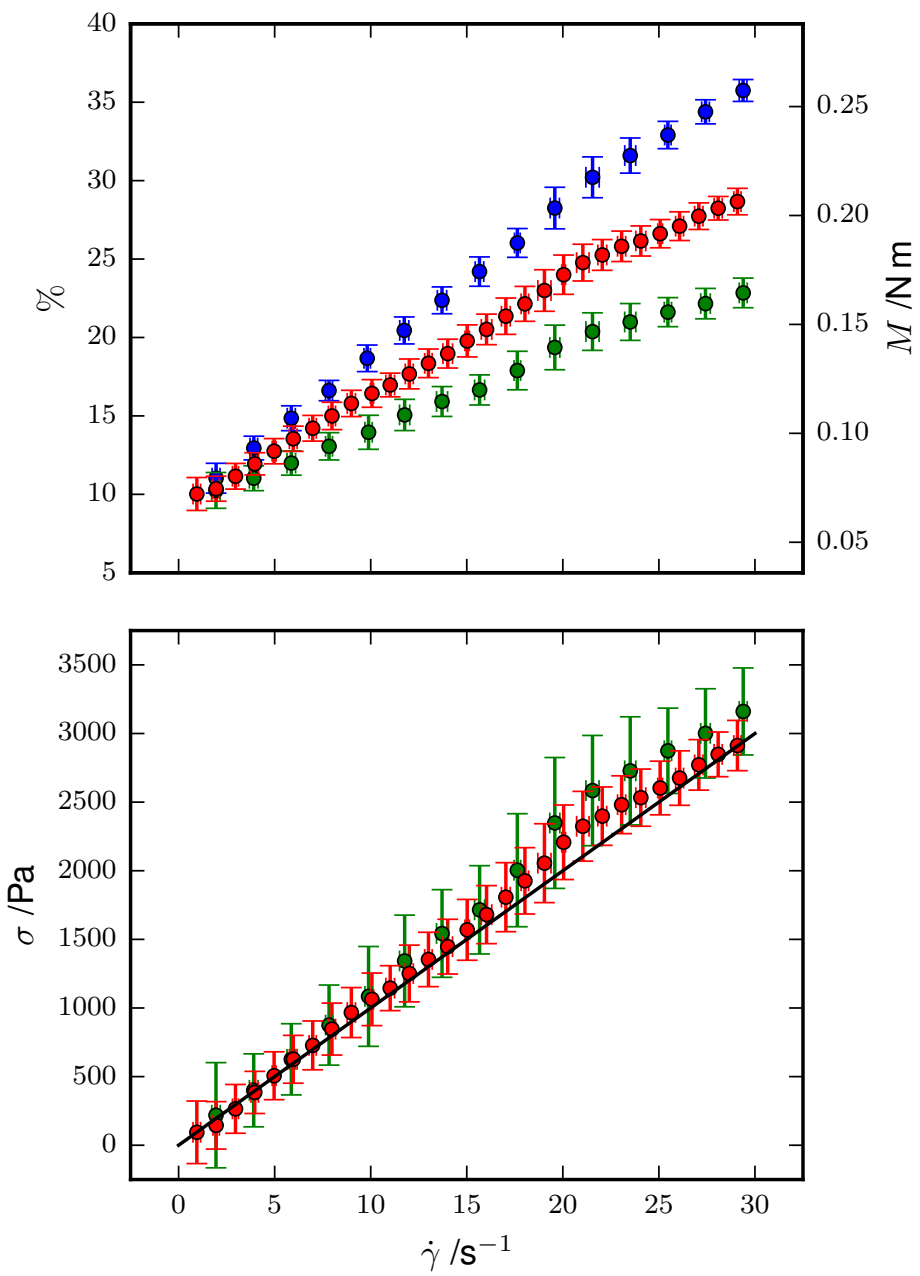

Figure 6.9: Uncorrected flow curves acquired using EvoDrive torque measurements and a 100k cSt sample at three fill heights (top); fill heights: 100 (॰), 71 (॰) and $45 \mathrm{~mm}$ (॰). Flow curves (45 and $71 \mathrm{~mm}$ ) after data was normalised with the $100 \mathrm{~mm}$ "calibration" data (bottom). 
represent the standard error of the shear stress values. The slope of the solid line is the known viscosity of the sample. Both datasets capture the expected viscosity within the reported error.

These proof of concept measurements were made on the bench-top, however, as the motor would be located outside the NMR magnet, it would be possible to collect the same data during NMR experiments. It should also be mentioned that the concentric cylinders and drive-shaft used for these experiments were not specifically designed for a torque measurement (i.e. simple PTFE bushings were used in both the drive-shaft and shear device). Furthermore, the rotor had a taper feature to maintain alignment with the cup. In addition to the fluid response the friction of these elements would have contributed to the overall torque measurement. Due to both the current resolution of the ST-23 torque measurements and the engineering of the drive-shaft and shear geometry a sample with a particularly high viscosity was used for these initial tests.

Looking at both the raw measurements and corrected flow curves in Figure 6.9 between 20 and $25 \mathrm{~s}^{-1}$ there is evidence of some anomalous behaviour in the measured value. As the current torque measurement resolution is not fine enough for use as a rheometer (for the types and size of samples which are currently studied with NMR) this artefact was not further explored.

\subsection{Integrated Analogue Torque Sensor}

Based on the measurements described above the potential for a torque measurement from the servo-stepper motor package is extremely exciting. Such a device would provide for a dynamic range of torque measurements and open up the possibility of stress controlled NMR shear experiments. However, significant work is still necessary (in drive system electronics, motor hardware and the mechanical design of the drive-shaft) to develop these methods. In the case of steady strain controlled experiments an inline torque sensor can be used to calculate dynamic viscosity (the design of this device was presented in Section 5.3.3). In this section, various aspects of the torque variant driveshaft unit will be explored including a discussion of error and sensor drift. Proof of concept data from the bench-top will be presented in addition to measurements made within the NMR magnet during experiments on water and a shear-banding wormlike micelle solution.

\subsubsection{Data Sampling and Averaging}

If torque measurements are to be made during an NMR experiment it is important to know the time over which the torque signal must be averaged in order to capture the 
true behaviour of the material under examination. In this section different sample times will be explored to probe the minimum torque measurement time.

For these experiments a silicone oil with nominal kinematic viscosity of $60 \mathrm{k}$ cSt (PN 181838, Sigma-Aldrich) was used. All measurements were made on the bench-top with the sample temperature maintained at $25^{\circ} \mathrm{C}$ using a water bath. A total of 14 stepped flow experiments were conducted with data points collected at 25 shear rate values logarithmically spaced from 0.1 to $25 \mathrm{~s}^{-1}\left(r_{\mathrm{i}}=8 \mathrm{~mm}\right.$ and $\left.r_{\mathrm{i}}=9 \mathrm{~mm}\right)$. The sampling time at each shear rate was varied between $1 \mathrm{~s}$ to $5 \mathrm{~min}$. For each of the 14 experiments the flow curve measurements were used to calculate a viscosity based on a linear fit with intercept set to zero. The determined viscosity (along with the standard error of the fit) as a function of averaging time can be seen in Figure 6.10.

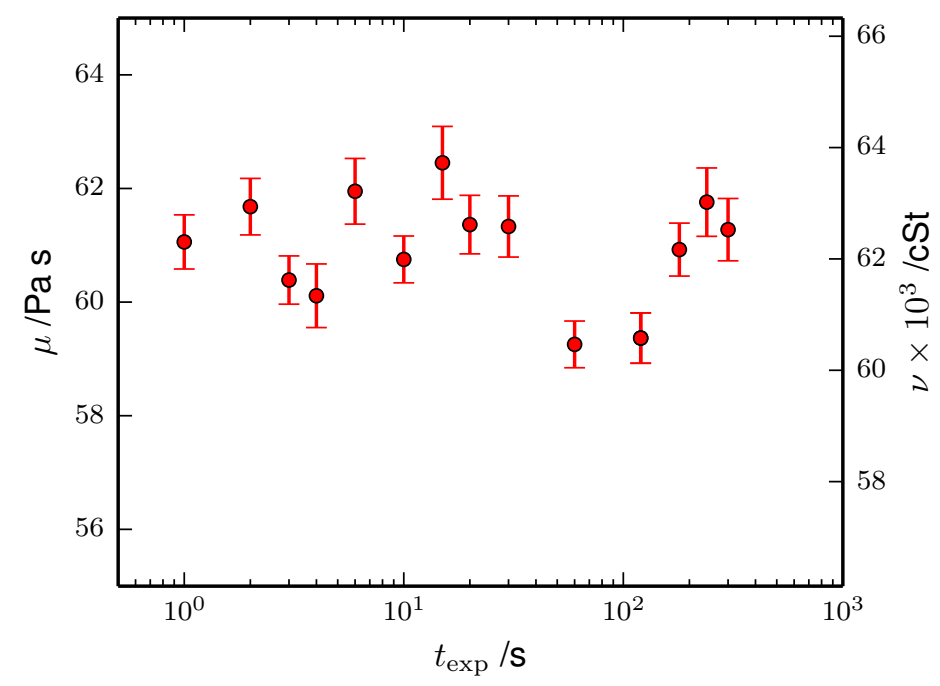

Figure 6.10: Viscosity calculated from flow curves acquired as a function of experiment time. The experiment time $t_{\exp }$ lists the length of data acqusition at each strain rate step.

A discussion of the accuracy of similar viscosity measurements will come later (Section 6.4.4) and for now it is assumed that the viscosity values reported in Figure 6.10 capture the actual fluid viscosity. This assumption is not unreasonable as the nominal viscosity is $60 \mathrm{kcSt}$ and all measurements are within approximately $5 \%$ of this value. In fact, regardless of the averaging time all measurements are within an envelope of 60-64k cSt and there appears to be little difference in the determined viscosity between the averaging extremes of $1 \mathrm{~s}$ and $5 \mathrm{~min}$. 


\subsubsection{Background Torque}

When discussing the design of the drive-shaft unit with the integrated torque transducer (Section 5.3.3) it was described that there is a shaft of approximately $300 \mathrm{~mm}$ between the shear geometry and the transducer. This length of drive-shaft is aligned with two bearings which will contribute a torque to the measured value due to internal friction. Furthermore, mechanical misalignment in the construction of the drive-shaft or coupling to the torque sensor could add a periodic torque as the shaft completes a full rotation. Characterising this background torque helps to identify the minimum error in a measurement and ultimately identifies the lower limit of measurable torques. Furthermore, it is important to know how the internal torque of the drive-shaft unit changes with the applied motor frequency.

To address these concerns a stepped flow experiment was conducted on the benchtop without a sample. Torque measurements were averaged over $20 \mathrm{~s}$ at 50 shear rates from 0.1 to $28 \mathrm{~s}^{-1}\left(r_{\mathrm{i}}=8 \mathrm{~mm}\right.$ and $\left.r_{\mathrm{o}}=9 \mathrm{~mm}\right)$. In Figure 6.11 the torque data are presented as a function of applied shear rate.

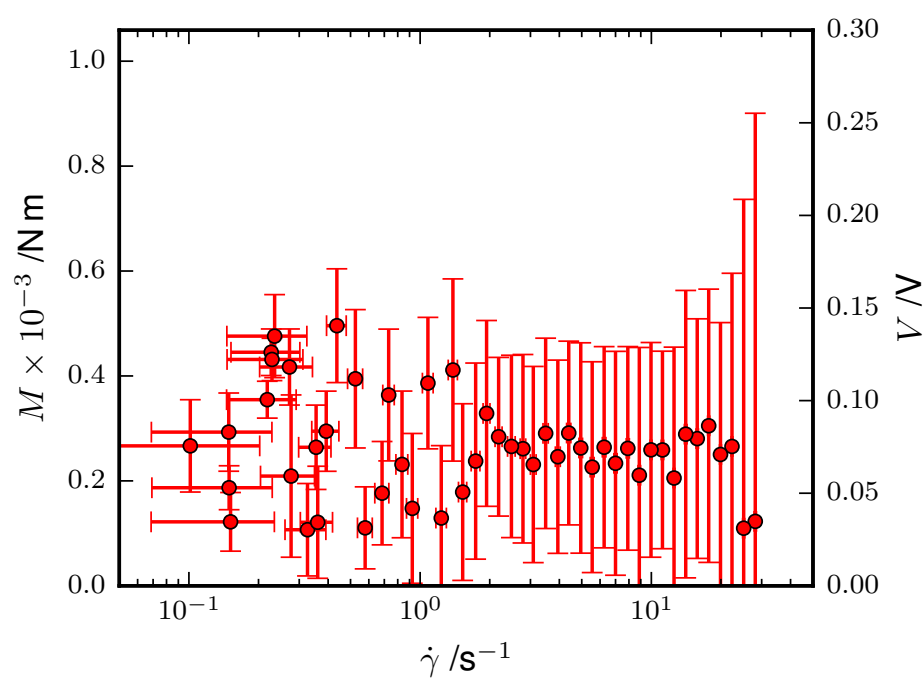

Figure 6.11: Torque data to show the magnitude of background torque as a function of rotation rate.

For the torque transducer, Vibrac TQ Mini 5.0, the stated error is $0.5 \%$ of the full range which, in terms of torque, is approximately $0.2 \times 10^{-3} \mathrm{Nm}(0.05 \mathrm{~V})$. This value is the approximate magnitude of the standard error of the measurements reported in Figure 6.11 and suggests that the design of the drive-shaft unit and the electronics do not act as a significant source of uncertainty in the bulk measurements.

Prior to collecting the data in this section the torque signal was zeroed while the 
shaft was stationary. All torque measurements shown in Figure 6.11 are within $0-0.6 \times 10^{-3} \mathrm{~N} \mathrm{~m}(0-0.15 \mathrm{~V})$. To reduce the error in measuring the torque response of a fluid the system could be zeroed while moving; however, this would have to take place before the sample was loaded. Regardless, based on the presented measurements and experimental procedure the torque error in the system due to the mechanical design is approximately $2 \%$ of the full range of the transducer.

Furthermore, the standard error in the applied shear rates (horizontal error bars) suggests that the drive-shaft has a lower limit of approximately $0.5 \mathrm{~s}^{-1}(0.01 \mathrm{~Hz})$.

\subsubsection{Stability and Drift}

To examine the stability of the transducer and electronics the torque signal was recorded over a 7 hour period. In this bench-top experiment the drive-shaft was not moving. After sitting unpowered at room temperature for a long period (i.e. overnight) the instrument was powered on and immediately the torque signal was zeroed. Afterwards, the torque and reference channels were continuously measured as a function of time.

The collected data can be seen in Figure 6.12 where the top panel shows the raw torque signal and the middle panel the reference signal. According to the user manual for the Vibrac torque interface card [137] the transducer should be allowed to warm up for 30 minutes prior to use ${ }^{2}$. Thus in actual experiments data would not be collected in this initial half hour and therefore these measurements have been excluded from Figure 6.12 and the subsequent analysis. To reduce the measurement noise a running average was calculated for both data sets (plotted as a solid black line in both panels). From this experiment it is apparent that the torque signal will drift over long periods. The drift trend in the torque and reference measurements is identical; for the data presented in Figure 6.12, if both sets of data are scaled and offset appropriately, the two measurements lay on top of each other.

To account for the drift of the torque signal the reference channel data could be used to correct measurements in post-processing. An example of this correction is shown in the bottom panel of Figure 6.12; the reference measurement (scaled by a constant) was subtracted from the torque measurement to correct for drift. The scaling constant used above must be determined empirically through an experiment for the environment in which torque measurements will be made (i.e. ambient temperature in laboratory or within thermal environment of NMR magnet bore). Alternatively, in lieu of a postprocessing correction, the reference channel could be used as part of a closed-loop system where the zeroing signal is varied in real-time to maintain the reference channel at a fixed value, however, this strategy has not yet been explored.

\footnotetext{
${ }^{2}$ Unless otherwise stated all analogue torque measurements in this thesis were made at least 30 minutes after powering on the Vibrac transducer.
} 

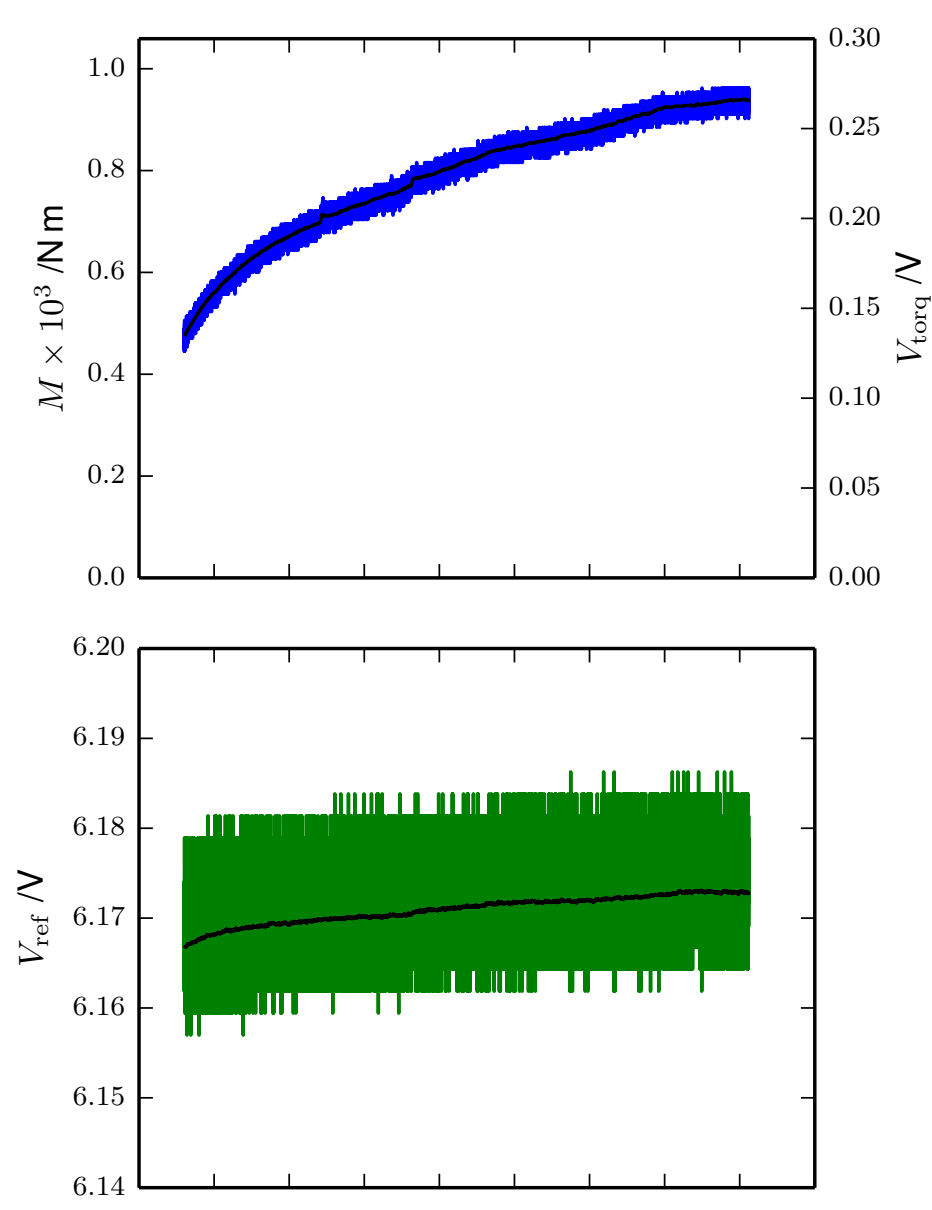

Figure 6.12: Raw torque (top panel) and reference (middle panel) voltage signals; for both sets of data a running average has been calculated (black trace). Using the reference signal the torque data was corrected for drift. The bottom panel compares the raw torque signal (blue trace) to the drift compensated torque data (red trace). 
Alternatively, drift could be reduced by re-zeroing the signal. However, in the case of series of stepped flow measurements, it may not be feasible to zero the electronics between each experiment. Using the data in Figure 6.12 to imagine a worst-case scenario, if the instrument ran for one hour (in which it is not possible to zero the sensor) and the experiment was started immediately after the warm-up period (when the greatest drift is observed) the drift would be approximately $0.04 \mathrm{~V}(0.4 \%$ of the measurement range). This is less than either the error of transducer or the error due to the design of the instrument.

\subsubsection{Benchmark to Commercial Device}

Based on the data presented in the two previous sections (6.4.2 \& 6.4.3) the measurement error of the drive-shaft unit with the inline torque sensor due to the mechanical design and electronic circuits is $\approx 2 \%$ of the full torque range of the Vibrac transducer with measurement drift potentially contributing an additional $\approx 1 \%$ error. However, even with an estimate of the torque measurement error it can still be difficult to estimate the error of a bulk rheological parameter (e.g. viscosity). To further probe the measurement accuracy of this instrument stepped flow experiments were made on three different silicone oils where the viscosity calculated from the torque measurements was compared to the viscosity determined by a commercial rheometer (TA Instruments AR 2000).

The three silicone oils used are listed in Table 6.1 along with the viscosity as measured by a commercial rheometer (TA Instruments AR 2000). The commercial rheometer measurements were made at $25^{\circ} \mathrm{C}$ with a $60 \mathrm{~mm} 2^{\circ}$ stainless steel cone and plate geometry. The drive-shaft unit measurements were conducted on the bench-top with a concentric cylinder geometry $\left(r_{\mathrm{i}}=8 \mathrm{~mm}\right.$ and $\left.r_{\mathrm{o}}=9 \mathrm{~mm}\right)$ and the sample temperature maintained at $25^{\circ} \mathrm{C}$ by a constant temperature water bath. In setting up the shear cell a $0.5 \mathrm{~mm}$ shim was always used to space the rotor from the bottom of the cup; as the truncation gap of the cone on the rotor was $60 \mu \mathrm{m}$ this spacing would have violated the assumption that the bottom of the rotor behaved as a cone and plate geometry. However, as the treatment of end effects in concentric cylinder geometries, as discussed in Section 2.4.2, assumed a narrow gap $(\kappa \geq 0.99)$ the use of Equation 2.130 is only an approximate correction. Viscosity was calculated from the applied shear rate and shear stress where Equation 2.130 was used to calculate the stress based on the physical dimensions of the rotor.

Table 6.1 lists both sets of viscosities and the percent error. The individual measurements can be seen in Figure 6.13 were the error bars represent the standard deviation of the torque measurement (converted to a shear stress) and the slopes of the dashed lines are equal to the viscosity determined by the commercial rheometer. 


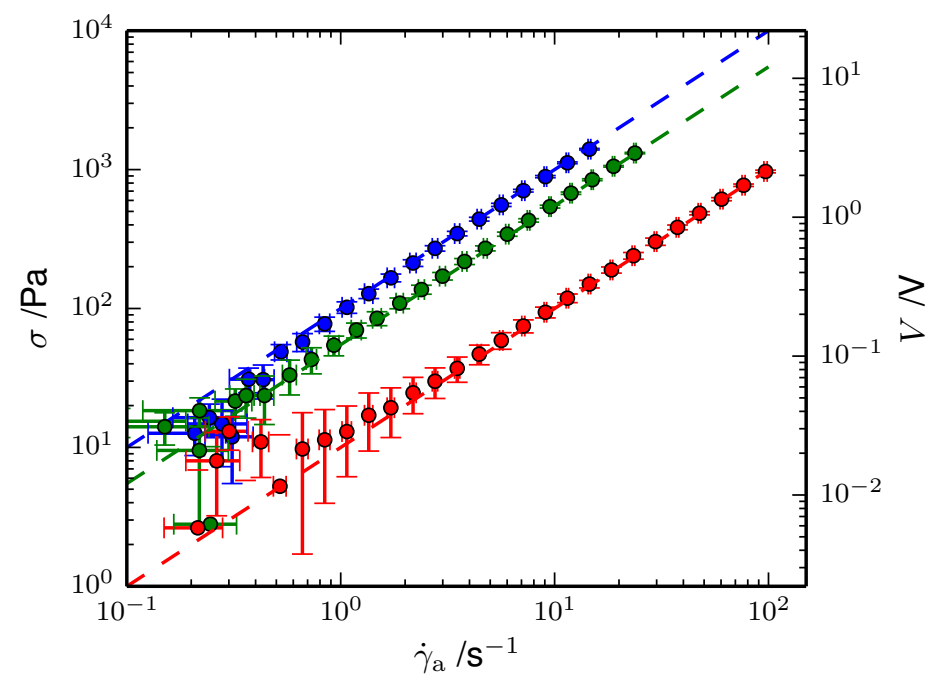

Figure 6.13: Rheology flow curves for three silicone oils, measured by NMR compatibile rheometer (markers) and compared to viscosity determined from commercial rheometer (dashed lines); nominal viscosities: 10k (॰), 60k (॰) and 100k cSt (๑).

The reported percent errors in Table 6.1 may seem high considering the uncertainty in the torque measurement is approximately $3 \%$, however, in calculating viscosity there is a noticeable second source of error. For the concentric cylinder geometry shear stress can be calculated from torque using Equation 2.109 (ignoring for the moment the influence of end effects). Combining this equation with the viscous model of Newtonian behaviour (Equation 2.60) and solving for viscosity yields:

$$
\eta=\frac{M}{2 \pi r_{\mathrm{i}}^{2} l \dot{\gamma}}
$$

Assuming all parameters are independent and the only source of errors is the torque $M$ and sample fill height $l$, the uncertainty in the viscosity $s_{\eta}$ can be approximately defined by

$$
\frac{s_{\eta}}{\eta} \approx \sqrt{\left(\frac{s_{l}}{l}\right)^{2}+\left(\frac{s_{M}}{M}\right)^{2}}
$$

\begin{tabular}{cccc} 
Sigma-Aldrich PN & Known Viscosity /Pa s & Measured Viscosity /Pa s & \% Error \\
\hline 378402 & 9.99 & 10.68 & 6.9 \\
181838 & 54.91 & 59.26 & 7.9 \\
378437 & 99.96 & 102.98 & 3.0
\end{tabular}

Table 6.1: Viscosity data for three silicone oils measured by a commercial instrument and the drive-shaft unit with inline torque transducer. 
The relative error of the torque measurement $\frac{s_{M}}{M}$ has already been discussed and is on the order of 0.03 ; however, an estimate of uncertainty for fill height will now be explored.

For the three stepped flow experiments presented in Figure 6.13 the concentric cylinder geometry was nominally filled to $50 \mathrm{~mm}$. If this height were to vary $\pm 2 \mathrm{~mm}$ the relative uncertainty $\frac{s_{l}}{l}$ would be 0.04 . For a height of $2 \mathrm{~mm}$ the volume within the annulus over this length $v=\pi l\left(r_{\mathrm{o}}^{2}-r_{\mathrm{i}}^{2}\right)$ would be approximately $0.1 \mathrm{~mL}$. Although the utmost care was taken in measuring out the appropriate sample, due to the narrow diameter and long length of the concentric cylinder geometry, it is possible that a small portion of sample $<0.1 \mathrm{~mL}$ may stick to the wall of the cup and not join the continuous fluid domain. Looking back at Equation 6.4 the resulting uncertainty in the viscosity would be on the order of $5 \%$. When considering the uncertainty in the torque measurement and the fill height the percent error of the measured versus known viscosity for each sample in Table 6.1 seems appropriate.

Finally, all three of the estimates of viscosity from the drive-shaft were higher than expected. There may be a systematic error due to an offset in the torque signal (i.e. at zero torque the voltage was non-zero). In subsequent experiments the firmware was updated so that before starting a stepped flow series of measurements the torque signal is measured over a short period (e.g. $30 \mathrm{~s}$ ) while the drive-shaft is stationary. This provides a baseline torque signal that can be subtracted from all measurements. This modification allows for agreement with data from a commercial rheometer (e.g. Figure 6.14) even for samples with a viscosity much less that the silicone oils used in this benchmark test.

\subsubsection{Simultaneous Torque and NMR Data Acquisition}

In discussing the performance of the drive-shaft unit with the integrated torque transducer (previous four sections) all measurements thus far have been made on the benchtop. As the main purpose of this instrument is simultaneous NMR acquisition and bulk rheometry measurements this sections explores the results of torque measurements made within the magnet during 1D NMR velocimetry experiments.

Two samples were used in these simultaneous measurements: a silicone oil with a viscosity of $60 \mathrm{kcSt}$ (also used in Sections 6.4.1 \& 6.4.4) and a wormlike micelle solution (discussed in Section 7.2). The silicone oil was selected as a calibration fluid with Newtonian behaviour while the response of the wormlike micelle solution is nonNewtonian.

The 1D NMR velocimetry used for these experiments was the same as the approach described in Section 6.2.1. Furthermore, the torque signal acquisition and subsequent calculation of shear stress followed the methods previously described in Section 6.4. 
The results from both sets of simultaneous experiments can be seen in Figure 6.14.

The top two panels of Figure 6.14 display the measurements on the silicone oil. The top left panel is the rheology flow curve where the shear stress is plotted as a function of shear rate. The error bars depict the standard deviation of the measured torque (converted to shear stress) and the shaft rotation (converted to shear rate). A linear model with zero intercept was fit to the data and plotted as the dashed line showing the torque sensor within the magnet did capture the expected Newtonian behaviour. The top right panel shows the velocity profile across the fluid gap where the shading of the marker indicates the applied shear rate (light to dark shading is equivalent to low to high shear rates). The applied shear rate of each velocity profile can be determined from the corresponding flow curve. The two dashed lines help to visualise how the velocity profiles change with increasing shear rate and are strictly qualitative; these are straight lines which is reasonable as an approximation for the curvature of the concentric cylinder $(\kappa=0.89)$ used in these experiments.

The bottom two panels of Figure 6.14 show the results from the simultaneous measurements on the wormlike micelle solution. As will be described in Section 7.2, below a critical shear rate $\left(\dot{\gamma}<3 \mathrm{~s}^{-1}\right)$ this sample is known to have shear thinning behaviour while at higher shear rates the shear stress remains constant. This response can be seen in the bottom left panel where the shear stress is plotted as a function of shear rate. The green markers represent the data collected with the drive-shaft unit while the unfilled markers were measured with a commercial rheometer $\left(60 \mathrm{~mm} 2^{\circ}\right.$ stainless steel cone and plate geometry, TA Instruments AR 2000). Both stepped flow measurements of the wormlike micelle solution show reasonable agreement; this is particularly true around the transition to the stress plateau. This result is quite exciting considering the measurement range of the shear stress $(0-150 \mathrm{~Pa})$. The bottom right panel of Figure 6.14 contains the 1D velocity profiles obtained as a function of shear rate. As was the case for the silicone oil the shading represents the magnitude of the applied shear rate. Additionally, dashed lines have been plotted to help visualise the flow. In particular, at higher shear rates, a shear banding phenomenon is observed where two phases of differing viscosity co-exist in the sample. The shear banding observed in the velocity profiles corresponds to the shear stress plateau in the flow curve. The wormlike micelle system and response to deformation will be thoroughly introduced and explored in the following chapter. For now these measurements are simply presented to illustrate the ability of the instruments developed in this thesis to study complex fluids and their behaviour. 

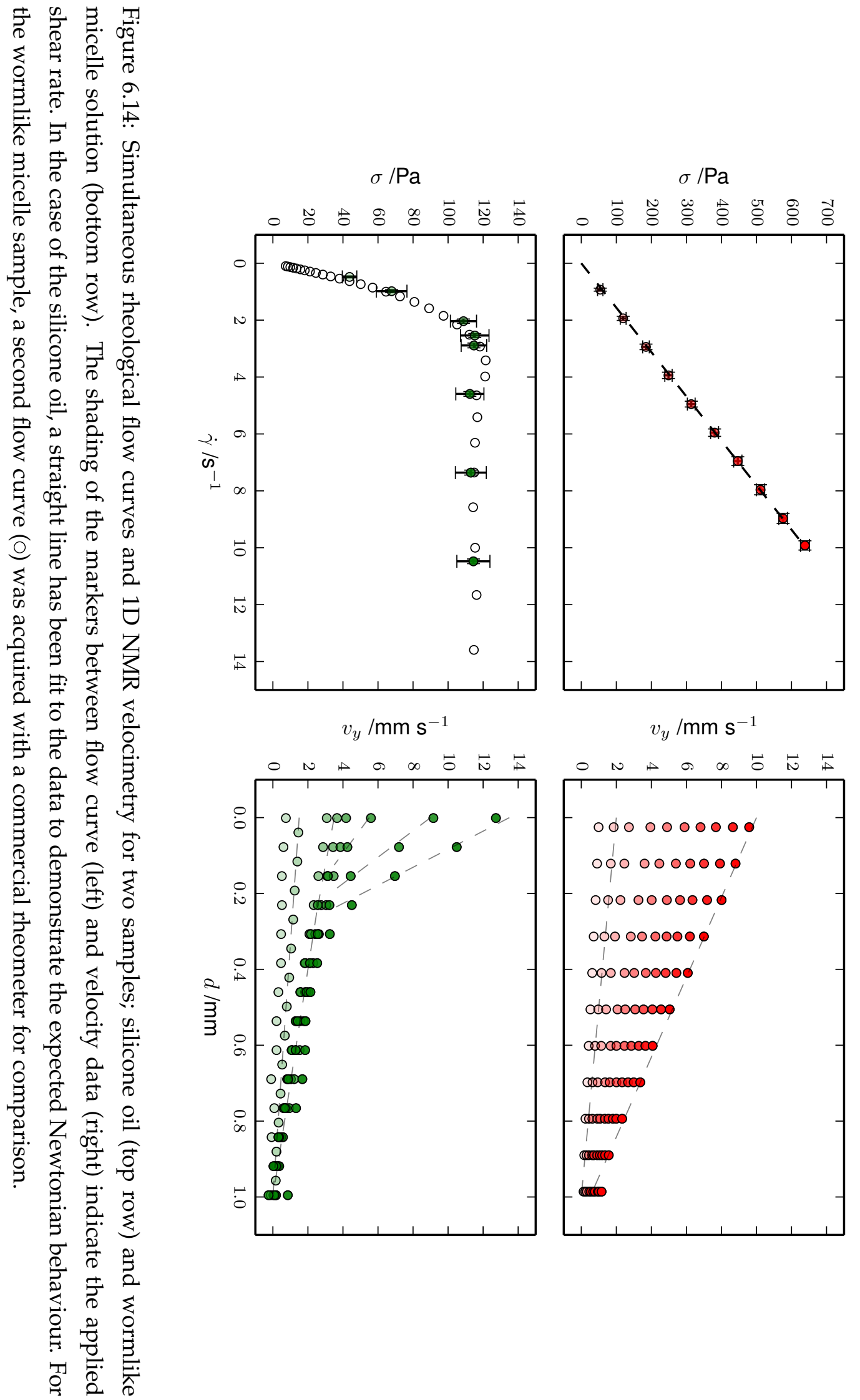


\subsection{Conclusion}

In this chapter, results from proof of concept experiments have been presented to demonstrate the operation and utility of the hardware designed in this thesis. Regarding the new shear geometries, 1D velocity measurements of water in various concentric cylinder devices agreed with theoretical predictions illustrating both the correct operation of the cells and the ability to explore the influence of curvature on the shear induced fluid flow. When examined side-by-side, 2D velocity maps acquired using the planar-cylindrical hybrid device nicely match results from numerical simulations. Furthermore, linear velocity profiles have been observed within the hybrid geometry, introducing planar Couette flow (simple shear) as a new deformation in the Rheo-NMR toolbox.

A variety of shear rate controlled motion profiles were presented, including examples of oscillatory waveforms and constant acceleration. These new experimental protocols can be used to explore aspects of rheology (e.g. large amplitude oscillatory shear) that have previously never been combined with NMR techniques and improve existing experiments such as shear quench (pre-shear) or start-up/cessation flow. Additionally, data was presented to illustrate the ability to synchronise the NMR acquisition with the motor.

In an effort to integrate bulk torque measurements into NMR experiments, feedback from the servo-motor was used to calculate the viscosity of a know sample. Using a calibration measurement, the servo-motor data was able to capture the actual viscosity of a silicone oil. However, using the current hardware, the resolution of such measurements was not sufficient for the complex fluids that would be studied in this thesis. Further refinement of this hardware or specific high torque applications could be the subject of future work. In lieu of servo-motor torque measurements, the performance of an analogue torque transducer was evaluated. After numerous bench-top tests to examine the uncertainty of bulk torque measurements from this drive-shaft unit, viscosity was measured of various samples. Finally, datasets were presented to demonstrate the simultaneous acquisition of NMR and bulk rheological measurements. 


\section{Chapter 7}

\section{Applications}

\subsection{Overview}

The hardware and methods developed in this thesis have the potential to open up numerous new avenues for studying the rheology of various physical systems.

The new concentric cylinder design which is compatible with the Bruker variable temperature system (Section 5.2.1) has been used with the original Magritek Rheo-NMR drive-shaft and controller by a fellow student, S. Kuczera, to study the shear banding phenomenon of the cetylpyridinium chloride $(\mathrm{CPCl})$ / sodium salicylate $(\mathrm{NaSal})$ in brine wormlike micelle system $[87,144]$. The compatibility with the temperature regulating hardware allowed results from NMR experiments to be directly compared to spatially resolved measurements acquired with ultrasonic velocimetry.

The prototype high resolution (narrow bore) concentric cylinder geometry (Section 5.2.1) was sent to colleagues in the Bio-NMR Laboratory at Massey University (Palmerston North, NZ) where an applied shear was used to orient proteins for structural analysis [146]. Despite the number of high resolution NMR systems in academia and industry around the world currently no commercial Rheo-NMR hardware is available for high resolution NMR systems.

The parallel disks geometry (Section 5.2.2) was used at Victoria University of Wellington by a visiting student, P. de Vrieze, to investigate wall slip via both NMR and benchtop rheometry measurements. In this study $2 \mathrm{D}$ velocity maps were acquired for both a simple (silicone oil) and complex fluid (wormlike micelle system) to explore the influence of gap height, edge effects and wall roughness. The parallel disks configuration is the preferred geometry for studying the presence and influence of slip as multiple measurements can easily be made as a function of gap height [1, sec. 5.5].

In this chapter three further applications of the hardware developed in this thesis will be explored. These include a study of the influence of curvature on a wormlike 
micelle system (Section 7.2), flow observations of granular material (Section 7.3) and shear induced structural transitions in a surfactant solution (Section 7.4).

\subsection{Influence of Couette Curvature on a Shear Banding Worm- like Micelle Surfactant System}

Aspects of this section have been submitted as "Observations of the influence of Couette cell curvature on shear banding in surfactant wormlike micelles" to the Journal of Rheology Special Issue on Shear Banding [147].

\subsubsection{Introduction}

The cetylpyridinium chloride $(\mathrm{CPCl})$ / sodium salicylate (NaSal) in brine wormlike micelle system has been extensively studied in the past two decades and is considered a model system to illustrate nonlinear mechanical response under steady shear flow conditions [148]. The rheological signature of shear banding in wormlike micelle systems was first observed experimentally by $\mathrm{H}$. Rehage and H. Hoffmann [149] as a stress plateau in the steady-state flow curve; the theoretical basis of the stress plateau was later provided by M.E. Cates and colleagues [150]. In more recent years, experimental work has used a range of methods to measure the local velocity profile. Initial experiments with nuclear magnetic resonance (NMR) methods provided direct imaging of flow during shear banding [151]. Additional flow studies utilising other techniques included particle image velocimetry (PIV) [152], photon correlation spectroscopy (DLS) [153] and ultrasound velocimetry (USV) [154].

For all the local velocimetry studies previously listed measurements were made on the $\mathrm{CPCl} / \mathrm{NaSal} /$ brine system under shear in a concentric cylinder (cylindrical Couette) shear device. In general, similar time-averaged velocity profiles have been observed with numerous techniques. These observations include homogeneous flow at low shear rates (before the stress plateau) followed by a stress plateau in which shear banding is observed and two distinct shear bands are evident. Next to the stationary wall (frequently the outer surface) a lower shear rate band exists that is comparable in value to the shear rate that marks the onset of the stress plateau. Simultaneously, a high shear rate band exists next to the inner moving surface. As the applied shear rate is increased through the stress plateau the dynamics of the local shear banding is thought to be described by a simple lever rule $\dot{\gamma}=x \dot{\gamma}_{\mathrm{H}}+(1-x) \dot{\gamma}_{\mathrm{L}}$ were $x$ represents the gap fraction although recent work suggests this model may not always apply [144, 155-157].

Various NMR methods have been previously used to study surfactant systems including NMR spectroscopy [158-161], relaxation times (i.e. $T_{1}$ and $T_{2}$ ) [162] or motion 
(e.g. diffusion or flow) [144, 161]. Furthermore, various Rheo-NMR techniques have been used to study other shear banding materials [163] including: suspensions of polymer core shell particles [164], multi lamellar vesicle onion textures [165], semi-dilute solutions of linear polymers [162] and star polymers [166].

With some notable exceptions [167] the majority of NMR experiments (in addition to other local velocity measuring techniques) that have explored shear banding of wormlike micelles have been conducted with a cylindrical Couette shear device. As discussed in Section 2.4.2 concentric cylinder geometries are used to create continuous Couette flow in order to approximate the ideal case where flow is induced in a sample held between two parallel plates by moving one plate (i.e. simple shear). For the cylindrical geometry, the infinite parallel plate treatment is increasingly relevant for cylinders with lower gap to radius ratios. However, for wide bore micro-imaging NMR magnets, the homogeneous region, in which measurements can be made, limits sample diameters to $\leq 30 \mathrm{~mm}$. Additionally, signal-to-noise considerations limit the size of imaging voxels which in turn motivates gap widths on the order of $1 \mathrm{~mm}$.

In the case of $\kappa \geq 0.99$, influences from curvature can be neglected and the shear strain (and shear rate) will be the same as the case of parallel plates (e.g. Equation 2.122). In the event that $\kappa<0.99$, a more sophisticated definition of shear rate was originally developed by Newton and later corrected in 1845 by G. Stokes [14, sec. 2.5]. This treatment requires torque measurements as a function of angular frequency and assumes ideal Couette flow (i.e. no secondary flow, and does not account for further errors from wall slip, shear heating or imperfections in the measurement device [1, pp. 190-195]). As a result the shear rate defined for the concentric cylinder geometry in Equation 2.122 is frequently used as an approximation even in studies where the $\kappa \ll 0.99$. Theoretical work by S.M. Fielding and H.J. Wilson [168] has suggested that Couette curvature could have an influence over flow and instability and warrants experimental investigation.

Earlier (Section 5.2.1) it was shown that for a "typical" Rheo-NMR geometry (assuming $r_{\mathrm{i}}=10 \mathrm{~mm}$ and $\left.r_{\mathrm{o}}=11 \mathrm{~mm}\right) \kappa=0.91$ and the shear stress of a Newtonian fluid increases by more than $20 \%$ across the gap. This suggests that the geometry does not ideally represent infinite parallel plates. Furthermore, it seems all local velocity techniques may lend themselves to a similar scenario where previous studies with PIV [152], DLS [153] and USV [154] used shear cells with $\kappa=0.89,0.88 / 0.96$ and 0.96 , respectively. These values are cited to illustrate the variety of cell designs and are not necessarily representative values for their respective techniques.

Local velocity measurements provide valuable insight into the dynamics in these systems; however, the shear stress variation in concentric cylinder geometries used in various studies makes it difficult to compare results. Recently, an intriguing study by S. Kuczera and colleagues [144] involved a tandem investigation of the $\mathrm{CPCl} /-$ 
$\mathrm{NaSal} /$ brine system using both Rheo-NMR and Rheo-USV. The geometries used in this study had $\kappa=0.89$ and 0.92 for the NMR and USV, respectively. Although the authors reported similar behaviour noticeable differences include structure in the vorticity direction and "bursts" observed via USV in temporally resolved 2D velocity maps of the gradient-vorticity plane that were not apparent in the NMR data. Despite the possibility for subtle differences in sample preparation and wall slip (due in part to shear device materials and manufacturing) the question remains: could minor differences in the geometry curvature (i.e. $\kappa$ ) lead to differences in the rheological response (in particular how instabilities manifest) of shear banding wormlike micelle systems? The present study explores this question by utilising new Rheo-NMR hardware to systematically study the curvature dependent response of the $\mathrm{CPCl} / \mathrm{NaSal} /$ brine system.

\subsubsection{Materials and Methods}

\section{Equipment}

NMR measurements on the range of shear cells were performed on a Bruker super wide bore superconducting magnet at a proton frequency of $300 \mathrm{MHz}$ with an Avance III spectrometer, a three axis imaging gradient system $\left(0.37 \mathrm{~T} \mathrm{~m}^{-1}\right.$ maximum gradient $)$ and a $60 \mathrm{~mm}$ RF coil. The simultaneous NMR-torque experiments were performed on a Bruker wide bore superconducting magnet at a proton frequency of $250 \mathrm{MHz}$ with an Avance III spectrometer, a Micro2.5 three axis imaging gradient system $\left(1.5 \mathrm{~T} \mathrm{~m}^{-1}\right.$ maximum gradient) and a $25 \mathrm{~mm}$ birdcage RF coil. For all experiments, temperature within the RF coil was controlled via the gradient system re-circulating bath set to $25^{\circ} \mathrm{C}$ which held the sample temperature within $\pm 1^{\circ}$ of the set-point.

The first device [129] was specially built for a Bruker super wide bore magnet, which has an enlarged room temperature bore and resonator coils much larger than those for the wide bore magnets typically used in Rheo-NMR studies (providing for samples up to $66 \mathrm{~mm}$ in diameter). Utilising the super wide bore magnet a series of concentric cylinder shear devices were constructed for this study and are represented in Figure 7.1. For all shear devices the cup was fixed while the bob rotated (Searle mode). The geometries were comprised of a glass tube and PEEK insert, which acts as the cup, and a solid PEEK rotor. The inner surface of the glass tube was not altered and the PEEK rotor was a "smooth" machined surface. Although no quantified description of the PEEK surfaces is available all cells were nominally machined to be identical and no macroscopic pattern was noted on any of the shear surfaces used in the present study. For both the water and $\mathrm{CPCl} / \mathrm{NaSal} /$ brine experiments described below each cell was filled to give a fluid height of $\approx 100 \mathrm{~mm}$ leaving an air fluid interface at the top of the sample. The geometries were placed in the NMR magnet such that the centre of the 


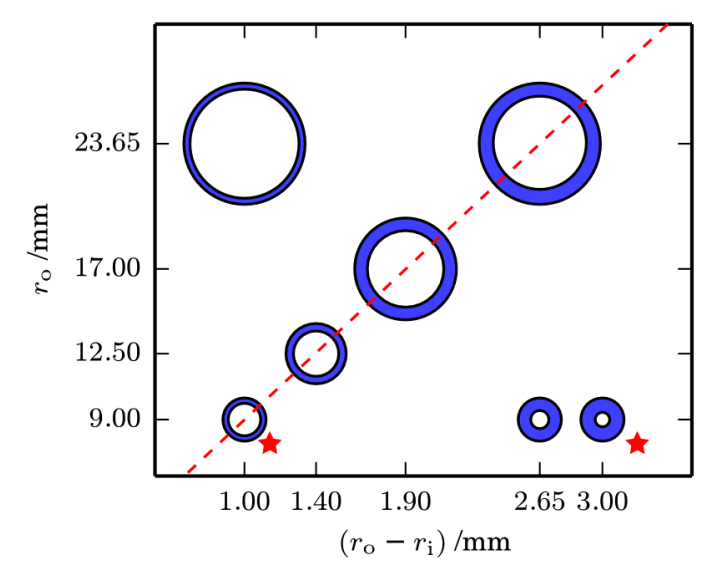

Figure 7.1: Concentric cylinder sampling matrix illustrating the various geometries used in the current study. The dashed line denotes shear cells with an identical stress variation across the gap. The two stars indicate the geometries with simultaneous torque measurements.

fluid domain was approximately aligned with the magnet centre.

The second device [130] was built to fit a standard Bruker wide bore magnet and included an inline torque sensor. When assembled this device is a stand-alone straincontrolled rheometer. The analogue torque sensor has a full measurement range of $35 \times 10^{-3} \mathrm{~N} \mathrm{~m}$. Both the cup and rotor of the shear cells used with the torque sensor drive-shaft were manufactured from PEEK. For these geometries, a nominal sample height of $70 \mathrm{~mm}$ was used and resulted in an air fluid interface at the top of the sample.

The design and construction of both assemblies was previously discussed in Chapter 5 with validation measurements presented in Chapter 6.

\section{NMR Methods}

A double slice selection 1D imaging sequence with velocity encoding was used to collect all data (Figure 6.1a). The motion encoding gradients and the encoding times varied depending on the expected velocity of the fluid with typical values for the encoding gradients $\delta$ of $1-3 \mathrm{~ms}$ and encoding periods $\Delta$ of $20-65 \mathrm{~ms}$. The NMR signal originated from the hydrogen nuclei and would be dominated by the bulk water molecules. Signal was recorded from a $10 \mathrm{~mm}$ slice in the vorticity direction which was $1 \mathrm{~mm}$ thick in the velocity direction. The $1 \mathrm{D}$ imaging was sampled along the velocity gradient direction (as illustrated in Figure 6.1b). These NMR parameters are such that there is no significant outflow of excited spins from the imaging voxel over the course of the NMR experiment as described recently by S. Kuczera and colleagues [144]. All 1D ve- 
locity profiles had a spatial resolution of $59 \mu \mathrm{m}$. The velocity of the fluid in the encoded direction (approximately tangential due to size of slice relative to the rotor curvature) was calculated from the phase accumulation over successive NMR experiments. In post-processing, the image was reconstructed, where the component of velocity was mapped to each pixel and the final product masked to remove noise. The mask was constructed from a magnitude image in which any pixel with signal intensity below $50 \%$ of the average in the fluid was ignored.

\section{Simultaneous NMR and Torque Measurements}

All NMR measurements for a shear geometry with a given sample were collected analogous to a stepped flow strain controlled rheology experiment (i.e. the applied strain rate was increased in successive steps at a fixed time interval). For the experiments in this study the time interval between strain rate steps was equal to the length of the NMR experiment (approximately $60 \mathrm{~s}$ and $120 \mathrm{~s}$ for the simultaneous NMR and torque measurements with the $1 \mathrm{~mm}$ and $3 \mathrm{~mm}$ gaps, respectively; the SWB experiments took approximately $4 \mathrm{~min}$ ). The torque data collected during the NMR experiments were averaged over the experiment time for each applied shear rate and converted to a shear stress using Equation 2.130 as discussed in Section 6.4.4.

\section{Laboratory Rheometry}

Simultaneous torque measurements were compared to steady state flow experiments performed on a TA Instruments AR-G2 rheometer equipped with a $60 \mathrm{~mm} 2^{\circ}$ steel cone and plate geometry. For these experiments the shear rate was ramped in logarithmic steps ( 8 points per decade) from $0.1-50 \mathrm{~s}^{-1}$ and the sample was held at $25^{\circ} \mathrm{C}$. A sampling time of $10 \mathrm{~s}$ was used with a maximum equilibration time of $60 \mathrm{~s}$ which was always sufficient to reach steady state behaviour.

\section{Sample Preparation}

The surfactant wormlike micelle sample was made of 8.09 wt.\% $(0.238 \mathrm{M})$ cetylpyridinium chloride $(\mathrm{CPCl})$ and $1.9 \mathrm{wt} . \%(0.119 \mathrm{M})$ sodium salycilate $(\mathrm{NaSal})$ in distilled water with $0.5 \mathrm{M}$ sodium chloride $(\mathrm{NaCl})$ and $0.2 \%$ Magnevist (gadopentetate dimeglimine, Bayer). The Magnevist was used to reduce the $T_{1}$ of the sample, decreasing the amount of time required between successive NMR experiments. Flow curve measurements on a commercial instrument showed the added Magnevist had no noticeable influence on the bulk rheology. All salts were purchased from Sigma Aldrich. The CPCl was a monohydrate and the additional mass from the water molecule was accounted for during sample preparation. 


\subsubsection{Results and Discussion}

One dimensional velocity profiles of the $\mathrm{CPCl} / \mathrm{NaSal} /$ brine system under shear in different concentric cylinder geometries all showed homogeneous flow at low shear rates and a transition to two-phase shear banded flow once a critical applied shear rate was reached. Example velocity profiles of homogeneous flow and two phase flow are illustrated in Figure 7.2.

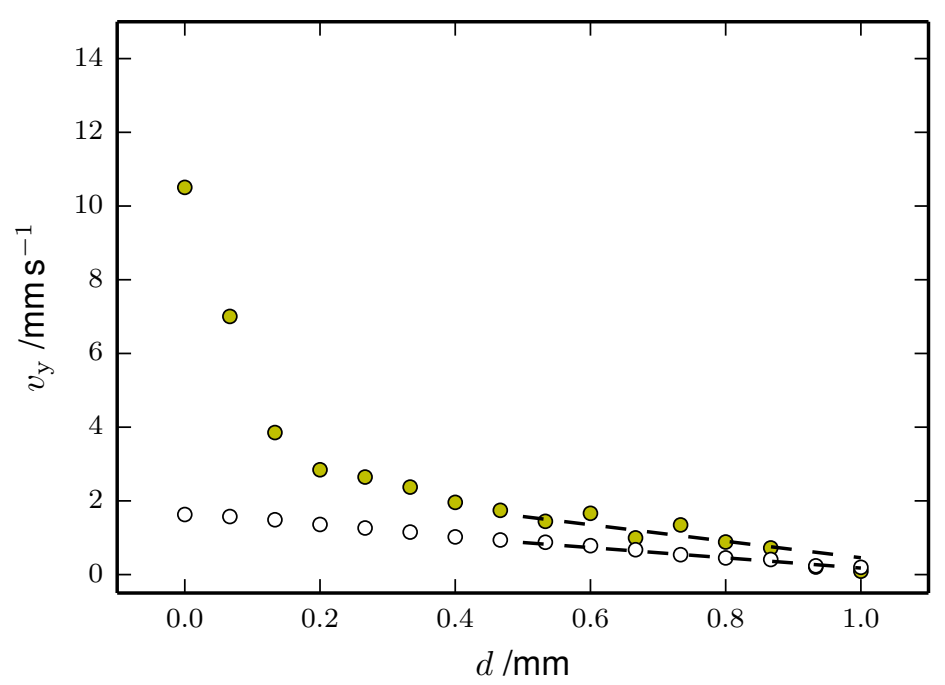

Figure 7.2: Example data for WLM before and after the onset of shear banding. These velocity profiles come from the shear cell with $r_{\mathrm{i}}=8 \mathrm{~mm}$ and $r_{\mathrm{o}}=9 \mathrm{~mm}$. The lower shear rate $\left(2 \mathrm{~s}^{-1} ; 0\right)$ demonstrates homogeneous flow while shear banded flow can be seen in measurements at $20 \mathrm{~s}^{-1}(\mathrm{O})$.

To characterise the local shear rate (prior to shear banding) or the shear rate in the low shear band (after the onset of shear banding) a linear fit was applied to the slowest half of the measured local velocity profile, i.e. adjacent to the stationary wall (demonstrated in Figure 7.2). Each NMR experiment measured fluid velocity across the entire shear cell yielding two sets of data (for the fluid gap on either side of the rotor). For a given experiment the local shear rate was an average of the fits for the two sides of the fluid gap in a single experiment. Fitting the slowest half of the velocity profile created a routine that could be applied to the range of shear rates presented in this study without risk of influence from the high shear rate band (individual fits were manually inspected to ensure no influence from the high shear band). It should be noted that based on the theoretical velocity profile for a Newtonian fluid (Equation 2.117) a linear fit does not precisely describe the local velocity profile; however, this approach was used as a method to generate an approximation for the local shear rate so comparisons 
could be made across geometries. The example fits in Figure 7.2 show little deviation of the actual data the linear model.

For the four Couette geometries with the same stress variation across the gap ( $\kappa=$ 0.89 ) the results of the linear fits to the slowest half of the measured velocity profile are plotted as a function of applied shear rate in Figure 7.3. A similar behaviour is seen in all geometries. Specifically, the local shear rate reaches a plateau around an applied shear rate of $2-3 \mathrm{~s}^{-1}$. The start of this plateau is interpreted as the onset of shear banding and coincides with the start of the stress plateau as seen in previous bulk rheology experiments $[144,169]$. Prior to shear banding, during homogeneous flow, the local shear rate determined from the measured velocity profile will increase as a function of the applied shear rate. However, after the onset of shear banding the shear rate in the low shear band becomes fixed. Detection of shear banding by the local shear rate of the low shear rate band is an alternative to directly observing two phase flow and may be a more sensitive approach as imaging resolution is much less important.

The same analysis shown in Figure 7.3 was used to compare the two Couette cells with the greatest difference in stress variation (Figure 7.4). In these experiments a difference was observed in the onset of shear banding (when the local shear rate as a function of the applied shear rate transitions to a plateau). For the geometry with the lowest stress variation $(\kappa=0.96)$ shear banding appears to begin around $4 \mathrm{~s}^{-1}$ while the onset of shear banding in the cell with the greatest stress variation $(\kappa=0.71)$ appears to have occurred before the first measurement at $1 \mathrm{~s}^{-1}$ (an observation supported by visually inspecting individual velocity profiles and confirmed by experiments reported below where NMR data was collected for shear rates below $1 \mathrm{~s}^{-1}$ ).

To further explore the curvature dependence of the onset of shear banding NMR velocity imaging experiments were performed with an NMR-compatible rheometer that allowed for simultaneous torque measurements. In this case, it was possible to measure the velocity profile at the same time and in the same geometry as the macroscopic stress response. Figure 7.5 shows the local shear rate extracted from the linear fit to the slowest half of the velocity profile for two Couette geometries. For the cell with the lower stress variation $(\kappa=0.89)$ analysis of the NMR velocity data suggested the onset of shear banding occurred at a shear rate around $2 \mathrm{~s}^{-1}$. This is in agreement (within experimental error) with other cells that have the same value of $\kappa$ (Figure 7.3) while the shear banding appears to begin around $1 \mathrm{~s}^{-1}$ for the cell with the greater stress variation $(\kappa=0.67)$. Apparent outliers in Figures $7.4 \& 7.5$ were not caused by experimental noise but are due to a deviation of the obtained velocity profiles from the simple two phase shear banding flow assumed in fitting data. This surprising flow behaviour may be due to temporal changes in the flow or secondary flows and will be the subject of future research. In a previous numerical study [168], curvature was found to be one factor 


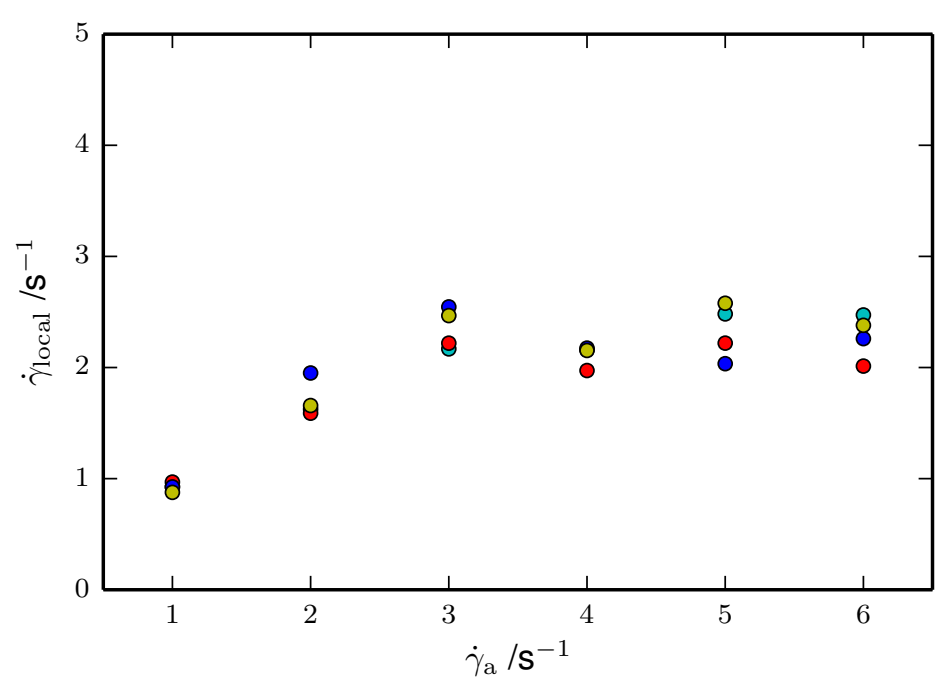

Figure 7.3: Local shear rate of the "slowest" portion of the fluid domain, i.e. the half of the fluid gap farthest from the rotating wall, in shear cells with the same stress variation. All Couette geometries had a $\kappa=0.89 ; \frac{r_{\mathrm{i}}}{r_{\mathrm{o}}}=\frac{8.00}{9.00}(\odot), \frac{11.10}{12.50}(\bullet), \frac{15.10}{17.00}(\bullet), \frac{21.00}{23.65}$ (०).

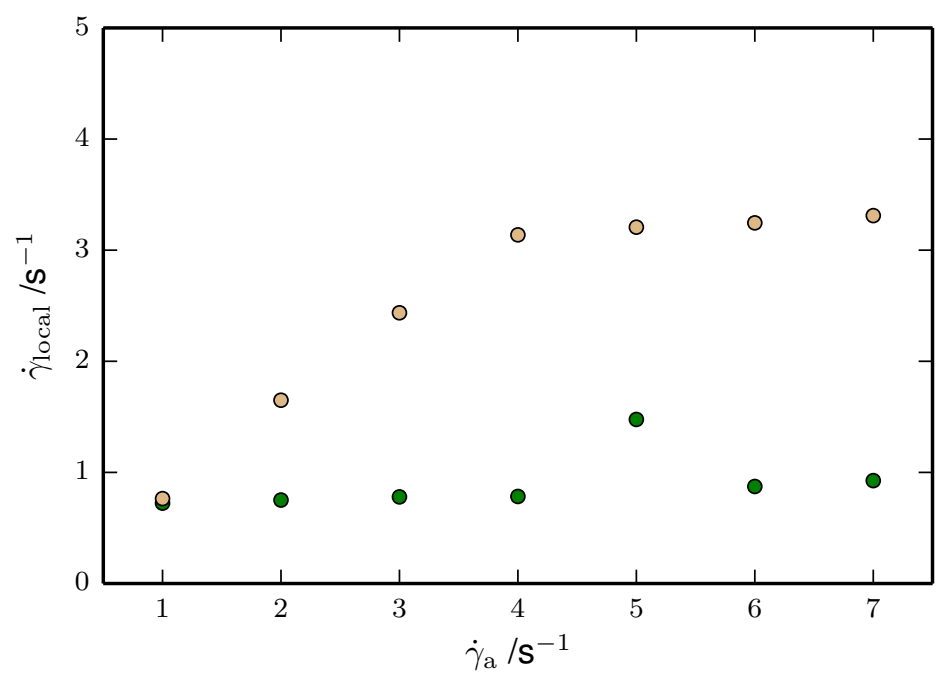

Figure 7.4: Local shear rate of the "slowest" portion of the fluid domain, i.e. the half of the fluid gap farthest from the rotating wall, in the Couette geometries with $\kappa=0.71$ (०) \& 0.96 (०). 


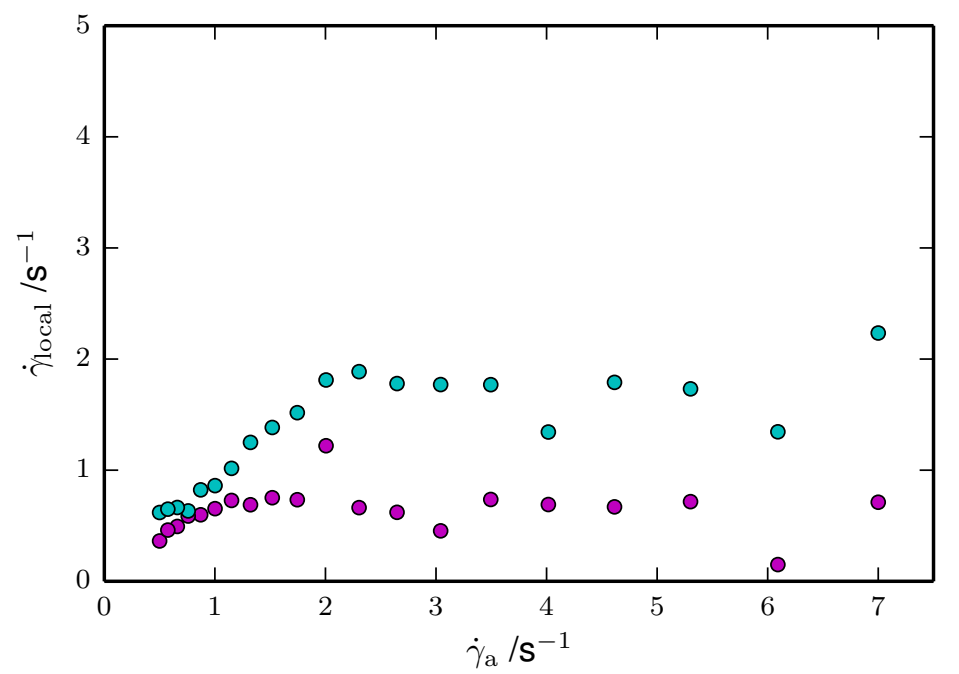

Figure 7.5: Local shear rate of the "slowest" portion of the fluid domain, i.e. the half of the fluid gap farthest from the rotating wall, in the Couette geometries with $\kappa=0.67$ (o) \& $0.89\left(r_{\mathrm{i}}=8.0 \mathrm{~mm} ; r_{\mathrm{o}}=9.0 \mathrm{~mm} ; 0\right)$. For these geometries, the NMR experiments were coupled with simultaneous torque measurements.

that influenced both the stability of the interface between shear bands and a bulk viscoelastic Taylor-Couette (VTC) instability. In this study the cell curvature was described by the parameter $q=\left(r_{\mathrm{o}}-r_{\mathrm{i}}\right) / r_{\mathrm{i}}$ and is analogous to $\kappa$ such that $q=(1 / \kappa)-1$. Furthermore, experiments were suggested on a range of concentric cylinder cells in the range of $q \approx 0.05-0.5$ [168] (or $\kappa \approx 0.67-0.95$ ), which follows with the hardware presented in this study. Future work could utilise fast velocity imaging techniques [170-172] to explore instabilities of primary and secondary flow.

In addition to the analysis of the NMR velocity data steady state flow curves of the bulk fluid response were constructed from torque measurements collected during the wide bore NMR experiments (Figure 7.6). In the bulk rheology flow curves, the sample with the lower stress variation $(\kappa=0.89)$ transitioned to a stress plateau at approximately $2 \mathrm{~s}^{-1}$ while the cell with greater curvature difference $(\kappa=0.67)$ entered a stress plateau at $1 \mathrm{~s}^{-1}$. Both of these are consistent with the onset of shear banding inferred from the velocity profiles.

For comparison, the bulk rheology of the same sample was measured on a commercial rheometer with a cone and plate geometry (described in Section 7.2.2) where the small angle approximation assumes no stress variation across the fluid domain. For all samples, the value of the shear stress across the stress plateau was equal. For the Couette geometry with $\kappa=0.89$ the bulk rheology nearly overlaps the cone and plate 


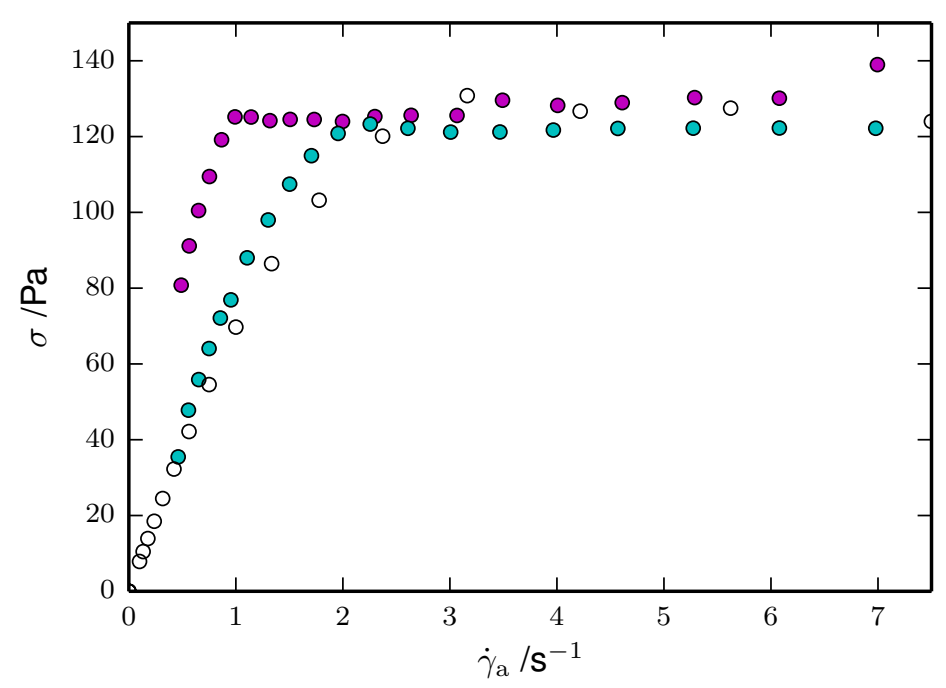

Figure 7.6: Flow curve data for commercial rheometer (o) and torque data collected simultaneously with 1D NMR velocimetry measurements, $\kappa=0.67$ (०) \& 0.89 ( $r_{\mathrm{i}}=8.0 \mathrm{~mm}$; $\left.r_{\mathrm{o}}=9.0 \mathrm{~mm} ; 0\right)$.

measurements. However, a noticeable shift is seen for the geometry with $\kappa=0.67$ and indicates the transition to shear banding has been shifted to lower shear rates compared to the other geometries.

Although not explicitly studied in the experiments presented here, the influence of wall slip certainly warrants discussion. Wall slip was evident for the homogeneous flow regime in individual velocity profiles as the pixel next to the moving rotor was lower than expected (in the validation studies with water, e.g. Figure 6.2, there was no deviation from the expected velocity for this pixel). Futhermore, the amount of slip in the homogeneous regime may not be an indication of the amount of slip during shear banding as wall slip has previously been shown as an alternative stress relaxation mechanism [173]. The question does remain: what is the interplay between shear banding, wall slip and geometry? However, this analysis is outside the scope of the current data.

\subsubsection{Conclusions}

This study included the first NMR velocity experiments acquired simultaneously with bulk rheology measurements. Furthermore, this was the first NMR study to systematically explore cylindrical Couette curvature. Both of these were enabled by new RheoNMR hardware.

The $1 \mathrm{D}$ NMR velocimetry and simultaneous NMR/torque measurements reported 
in this study demonstrate the importance of curvature in flow studies of the $\mathrm{CPCl} /$ $\mathrm{NaSal} /$ brine system. Specifically, the onset of shear banding and the stress plateau appear to vary as a function of Couette curvature. In the case of geometries with high stress variation between the two shear surfaces (low $\kappa$ ) shear banding in the $\mathrm{CPCl}$ $\mathrm{NaSal} /$ brine system, as indicated by a stress plateau as well as measured through NMR velocity imaging, occurred at lower applied shear rates. These measurements serve as evidence that comparisons between rheological studies must take care to consider the design and construction of the shear geometries utilised.

Continuing from these observations future work is necessary to explore the role of wall slip with Couette curvature and shear banding in the $\mathrm{CPCl} / \mathrm{NaSal} /$ brine system. Further work utilising the hardware introduced in this paper will also investigate (in)stability and secondary flow of shear banding wormlike micelles.

\subsection{Flow Observations of Granular Material}

The following section includes preliminary data from a collaborative study on the flow of granular materials [174].

\subsubsection{Motivation and Introduction}

Granular material is defined as a collection of discrete macroscopic particles. Natural examples include sand, soil and snow. Numerous industrial processes involve granular material with examples from agriculture (e.g. rice, sugar and seeds) to pharmaceutical production. Depending on the properties of the individual particles, overall volume concentration and applied external stimuli, granular materials can behave as either a solid, liquid or gas [175]. Despite the prevalence of these materials in both natural processes and global industries the rheology of these systems is still poorly understood [176, 177].

Numerous theoretical models have been proposed to describe granular flows. The geophysical community is particularly interested in dense particle concentrations where the granular system may be analogous to a viscoelastic fluid with a yield stress behaviour [178]. While at dilute concentrations techniques similar to the kinetic theory of gases are used to describe the dynamics of flowing near-elastic particles [179]. However, all models require the input of emperical data to properly capture the rheology of granular systems.

As discussed throughout this thesis NMR is a non-invasive technique for studying materials under shear and has previously been applied to flowing or fluidised granular systems [180-183]. The hardware specifically developed in this thesis is well suited for these studies. In particular the super wide bore drive-shaft and shear devices can be 
tailored to the diameters of particles and the desired number of grains spanning the fluid domain. Furthermore, the torque resolution of the Eva Robotics servo-motor may be ideal for simultaneous bulk shear stress measurements while the Vibrac inline torque transducer could be used to study fluidised flow.

\subsubsection{Experimental Methods}

\section{Samples}

Four granular systems were explored in this study; the particles and their respective mean diameters are listed in Table 7.1. These particles were chosen for their spherical quality and the measurable NMR signal from their core.

\begin{tabular}{cccc} 
Sample & Mean Diameter $/ \mathbf{m m}$ & $\mathbf{r}_{\mathrm{i}} / \mathbf{m m}$ & $\mathbf{r}_{\mathrm{o}}-\mathbf{r}_{\mathrm{i}} / \mathbf{m m}$ \\
\hline Lobelia Seeds & 0.3 & 16.00 & 7.65 \\
Petunia Seeds & 0.5 & 16.00 & 7.65 \\
Vitamin E Capsules & $\approx 1$ & 15.10 & 8.55 \\
Mustard Seeds & $\approx 2$ & 11.10 & 12.55
\end{tabular}

Table 7.1: Mean diameter for particles and the dimensions of the concentric cylinder geometries used for studies of granular flow.

\section{Equipment}

NMR measurements were performed on the Bruker super wide bore superconducting system $\left({ }^{1} \mathrm{H}\right.$ Larmor frequency of $300 \mathrm{MHz}$ ) used to study the influence of curvature on the onset of shear banding in the $\mathrm{CPCl} / \mathrm{NaSal} /$ brine wormlike micelle system (Section 7.2.2).

Flow of the individual granular systems was generated within a concentric cylinder geometry. The dimensions of the shear cell used for each size of particle is listed in Table 7.1. To prevent slip at the particle-solid interfaces double sided tape was used to affix a single layer of particles to both the rotor and cup wall. As a $140 \mathrm{~mm}$ length of precision bore glass tubing was used as the cup wall the double sided tape could be easily installed (Figure 7.7a). Electrical tape was used to prevent slip between the top cap of the concentric cylinder device and the glass tubing (Figure 7.7b). By marking the location of the parts prior to starting the shear experiments it was possible to check for relative movement (slip) at the end of an experiment. For these preliminary measurements the tape was sufficient to prevent any slip.

For each granular system the concentric cylinder was rotated such that the tangential wall velocity was either 17.1 or $41.2 \mathrm{~mm} \mathrm{~s}^{-1}$. 


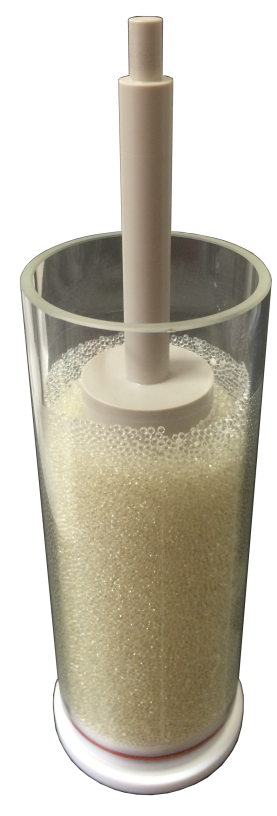

(a)

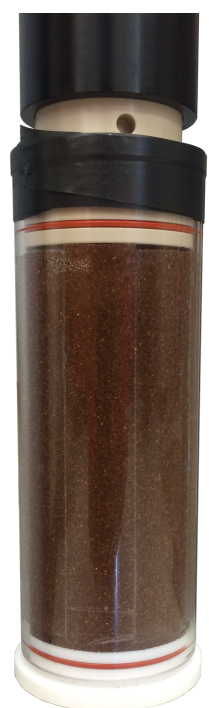

(b)

Figure 7.7: (a) Vitamin E capsules loaded in a concentric cylinder shear device (top cap not installed). The layer of seeds affixed to the glass tubing is visible at the top of the fluid domain. (b) Shear device loaded with lobelia seeds and mounted to super wide bore drive-shaft unit; electrical tape was used to prevent slip between the cap of the concentric cylinder and the glass tubing.

\section{NMR Methods}

A double slice selection 1D imaging sequence with velocity encoding was used to collect all data (Figure 7.8). Compared to other 1D motion imaging pulse programmes used throughout this thesis the refocusing $\pi$ pulse of the PGSE encoding portion was a hard pulse. Afterwards double slice selection was accomplished with two soft $\pi$ pulses and the NMR signal was acquired during the application of an imaging gradient.

The motivation behind a broadband (hard) $\pi$ pulse in the PGSE portion of the experiment was to access short observation times $\Delta$ over which fewer particle interactions would occur. The encoding time $\delta$ was fixed at $1 \mathrm{~ms}$ for all experiments. For each system multiple experiments were conducted as a function of the observation time $\Delta$ was varied between $1.8-7 \mathrm{~ms}$. For each experiment, $64 q$-gradient steps were used and ramped from $-95 \%$ to $92 \%$ of the max gradient strength $\left(0.37 \mathrm{~T} \mathrm{~m}^{-1}\right)$.

Signal was recorded from a $25 \mathrm{~mm}$ slice along the $z$ direction which was $3 \mathrm{~mm}$ thick in the velocity encoding $(y)$ direction. The $1 \mathrm{D}$ imaging was sampled along the velocity gradient $(x)$ direction (as illustrated in Figure 6.1b). 


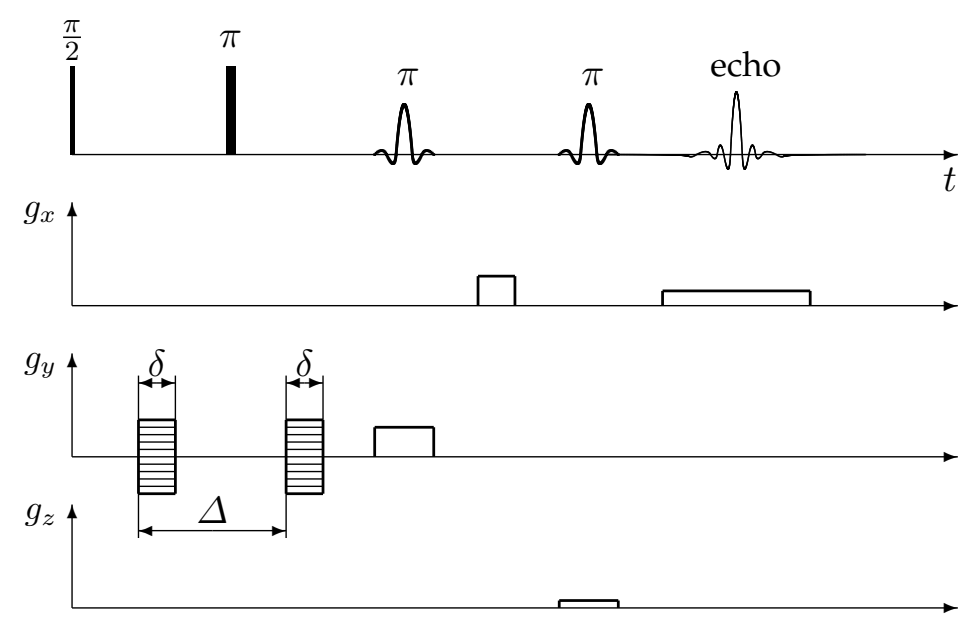

Figure 7.8: Pulse programme used for granular flow experiments.

The spatial resolution of all 1D images was $127 \mu \mathrm{m}$. The velocity profile of the fluid was calculated from the spatially dependent phase accumulation. In these calculations, only the centre 32 experiments in which the encoding gradient was ramped from approximately $-50 \%$ to $50 \%$ of the maximum strength were used. Additionally, the phase from an identical experiment where the motor was not moving was subtracted prior to converting the phase to a velocity. The edge of the fluid domains was identified from the magnitude image at zero gradient strength.

\subsubsection{Preliminary Results and Discussion}

Velocity profiles for the four particle systems can be found in Figure 7.9. Observations on the bench-top and the results of NMR experiments have shown that the concentric cylinder devices developed as part of this thesis are well suited to shear granular systems. The opportunity to tune the fluid domain to a specific particle has allowed various particle diameters $(0.3-2 \mathrm{~mm})$ to be studied.

With the hardware developed in this thesis it is possible to conduct a systematic study of various particles over a wide range of applied shear rates due to the first Rheo-NMR device specifically build for a Bruker super wide bore magnet. Spatially resolved velocity measurements, such as those presented in this section, could be used to describe the variance in particle velocities. By observing the variance of velocity as a function of observation time $\Delta$ it would be possible to estimate the mean collision time; at observation times less than the mean collision time the variance of velocity is constant. These mean collision times relate to the rheology and viscosity of the granular material [180] and would be of great use for refining theoretical descriptions of granular flow. Currently a detailed analysis is underway of the initial data collected with the 

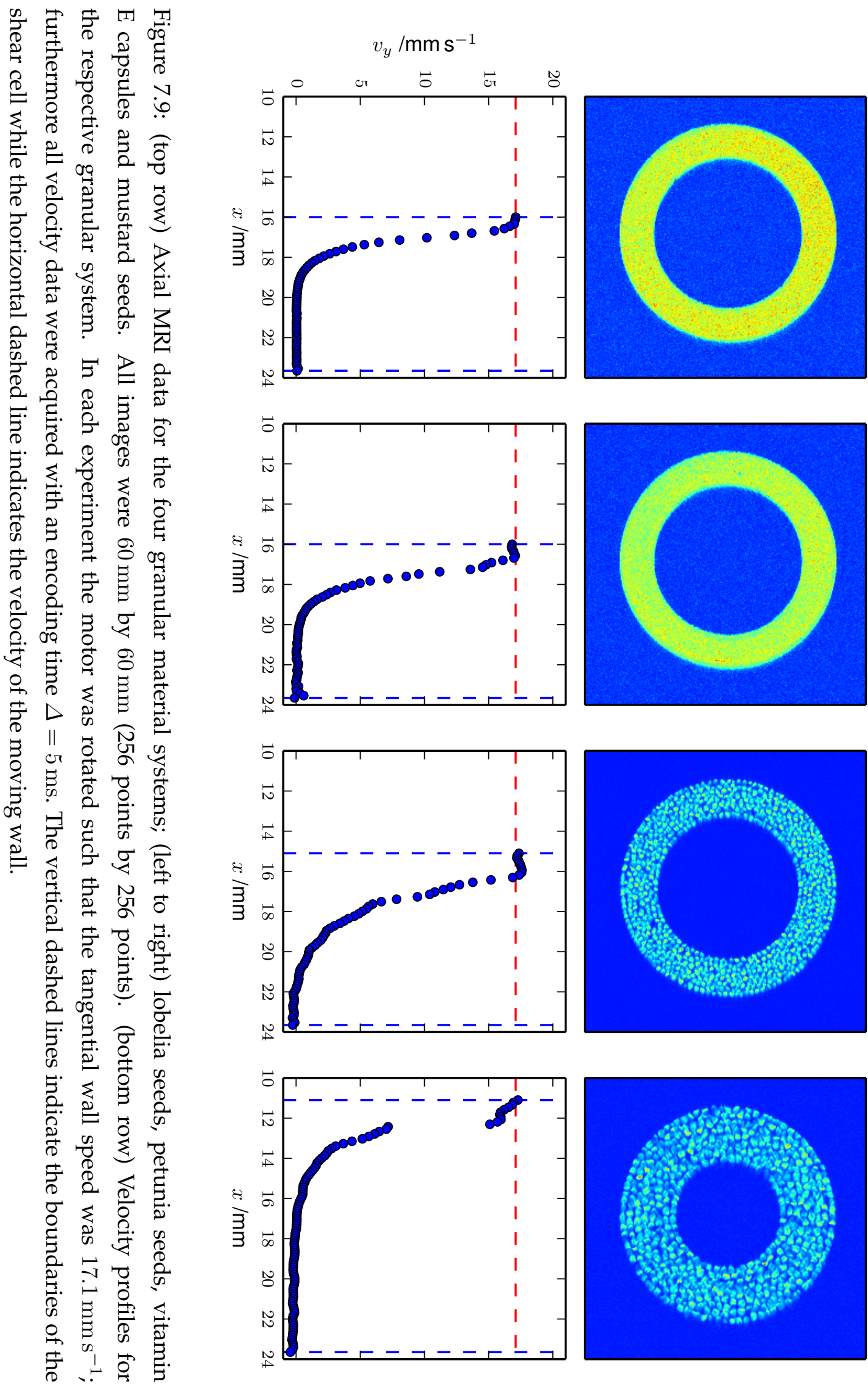
super wide bore drive-shaft unit with a forth coming manuscript submission [174].

\subsection{Shear Induced Structural Transitions in a Lyotropic Non- ionic Surfactant System}

This section demonstrates the ability to observe transient phenomenon via simultaneous measurements of deuterium spectroscopy and bulk viscosity.

\subsubsection{Introduction}

In recent years magnetic resonance techniques have frequently been used to study the shear induced phase transitions of surfactant systems [161, 184-187]. Of particular interest is the structural re-arrangement of nonionic surfactants such as the well documented triethylene glycol mono- $n$-decyl ether $\left(\mathrm{C}_{10} \mathrm{E}_{3}\right)$ in water; a dynamic phase diagram for this system has been published by C. Oliviero and colleagues [188].

\subsubsection{Experimental Methods}

A sample containing $40 \mathrm{wt}$. \% surfactant was prepared using triethylene glycol monon-decyl ether ( $\mathrm{C}_{10} \mathrm{E}_{3}$, PN BD-3SY, Nikko Chemical). The solvent was 9:1 $\mathrm{D}_{2} \mathrm{O}: \mathrm{H}_{2} \mathrm{O}$. The deuterium oxide was purchased from Sigma Aldrich (PN 151882). Chemicals were measured by mass, combined in a beaker and mixed overnight on a magnetic stir plate (no heat).

NMR measurements were made using a Bruker wide bore superconducting magnet at a proton frequency of $400 \mathrm{MHz}$ with an Avance spectrometer and a $25 \mathrm{~mm}{ }^{2} \mathrm{H}$ resonator coil.

The sample was loaded into the cup of the concentric cylinder and the total mass of sample was noted. Once the drive-shaft unit was installed in the NMR magnet the sample was conditioned at $42{ }^{\circ} \mathrm{C}$ and a shear rate of $10 \mathrm{~s}^{-1}$ for approximately one hour to establish the planar lamella phase (as identified by ${ }^{2} \mathrm{H}$ spectrum). Once the $\mathrm{L}_{\alpha}$ structure was established the motor was stopped and the temperature was lowered to $25^{\circ} \mathrm{C}$ where the sample was allowed to equilibrate for one and a half hours. Afterwards, the torque sensor was zeroed while the drive-shaft was stationary. Finally, simultaneously, the spectrometer began acquiring data as a constant shear rate of $10 \mathrm{~s}^{-1}$ was applied.

NMR data was acquired via successive single pulse experiments (Figure 3.6) with a sweep width of $2500 \mathrm{~Hz}$. The free induction decay was measured using 256 complex points and averaged over four successive scans. After the four scans of a single experiment were finished the spectrometer would repeat the experiment; a total of 400 NMR 
experiments were run with a complete experiment taking approximately $14 \mathrm{~s}$ (total experiment time approximately $95 \mathrm{~min}$ ). During the NMR acquisition, the torque signal was recorded at a sampling frequency of approximately $20 \mathrm{~Hz}$.

\subsubsection{Results and Discussion}

The real portion of the phase corrected NMR signal is plotted as a function of accumulated strain $\gamma=\dot{\gamma} t$ in Figure 7.10. The timing of each spectrum was determined from the time stamp of the NMR data file; for readability, only every fourth spectrum is displayed in the plot.

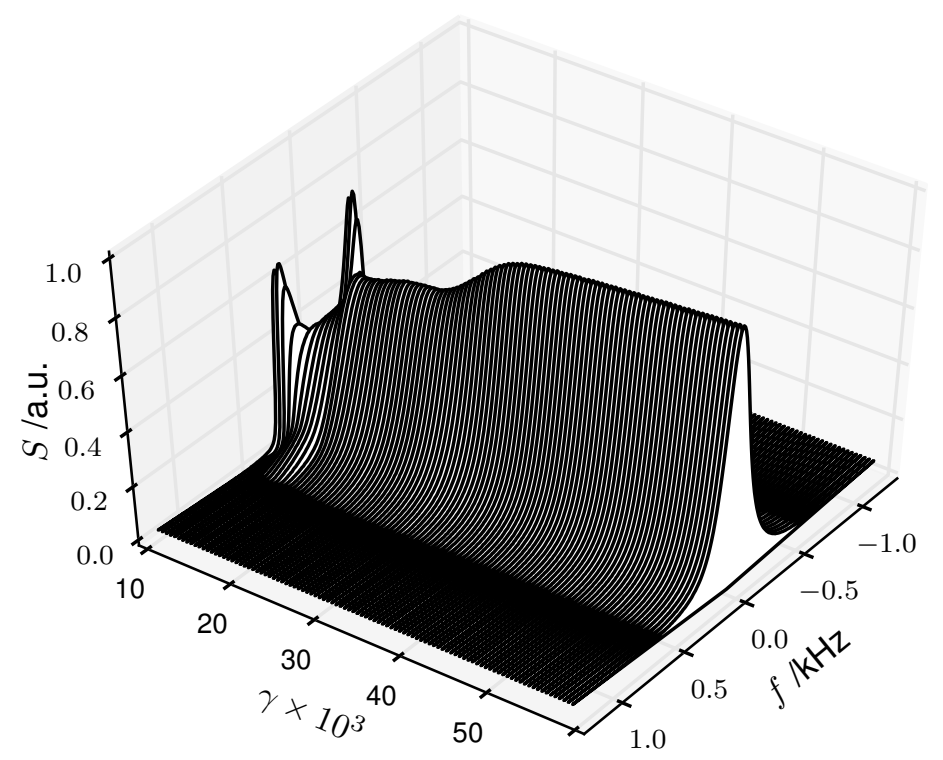

Figure 7.10: ${ }^{2} \mathrm{H}$ spectra collected as a function of accumulated strain.

The torque signal was converted to a shear stress using Equation 2.130 and used to generate a viscosity $\eta=\frac{\sigma_{12}}{\dot{\gamma}}$. To reduce noise a running mean was calculated using subsets of 280 points; based on the torque sampling frequency and length of a single NMR experiment this averaging period was approximately equal to the acquisition time of each spectrum. The transient viscosity data are plotted as a function of accumulated strain in Figure 7.11.

Both the ${ }^{2} \mathrm{H}$ spectra and viscosity measurements match published trends [185]. In particular, a shoulder is seen in the viscosity data (inset Figure 7.11, $\gamma=5.5 \times 10^{3}$ and $\eta=4.5 \mathrm{~Pa} \mathrm{~s}$ ) which was similarly observed in the published data. However, in this study (conducted at $25^{\circ} \mathrm{C}$ ) two plateaus are seen in the spectroscopy data once the sample had transitioned to the multilamellar vesicle structure which more closely follows the published data collected at $34^{\circ} \mathrm{C}$. Furthermore, the viscosity of the MLV phase (compared at $\gamma=16 \times 10^{3}$ ) in this experiment ( $13 \mathrm{Pas}$ ) is higher than the published 


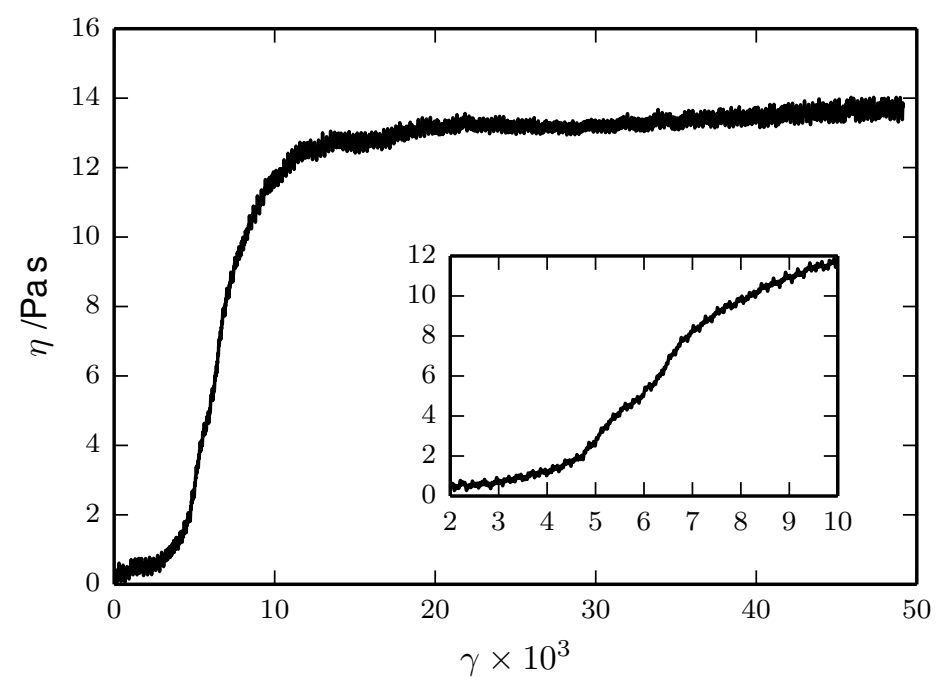

Figure 7.11: Transient viscosity as a function of accumulated strain.

value (9 Pas).

As the focus of this study was to illustrate the ability to simultaneously observe phase transitions with NMR and bulk rheometry, due to cost, old stock of $\mathrm{C}_{10} \mathrm{E}_{3}$ was used. The vial of surfactant was opened nearly four years earlier and had since been covered with Parafilm and stored at $2-4^{\circ} \mathrm{C}$. It is possible the properties of the surfactant could have changed during this time period. Regardless, the results illustrated that the hardware and methods developed in this thesis are suited to observe transient phenomenon (in addition to the steady state applications discussed earlier in this chapter). Furthermore, continued work on the $\mathrm{C}_{10} \mathrm{E}_{3}$ system by $\mathrm{S}$. Kuczera has involved using the oscillatory shear capabilities of the new drive system to perform large amplitude oscillatory shear (LAOS) Rheo-NMR experiments. The results of the LAOS work have subsequently been compared to equivalent LAOS studies using either conventional rheometry or small-angle light scattering (Rheo-SALS) [189]. 


\section{Chapter 8}

\section{Conclusion}

\subsection{Work Presented in this Thesis}

Within this thesis three key areas of hardware have been explored, including: 1) constructing NMR shear devices with geometric parameters closer to those used on commercial rheometers, 2) implementing an advanced drive system which allows for new shear profiles, including oscillatory shear and 3) integrating torque sensing into the Rheo-NMR experiment for simultaneous bulk shear stress measurements.

For torque measurements within the NMR magnet an analogue inline transducer was incorporated into a modified drive-shaft unit. Proof of concept studies have characterised the performance of this device as a strain controlled rheometer. Alternatively, the concept of utilising a servo motor to measure torque was explored. While the torque resolution of the current servo motor cannot currently compete with the stand-alone torque sensor these experiments have demonstrated the feasibility of an alternative torque sensor routine and the potential for stress controlled Rheo-NMR.

The drag flow shear devices discussed in this thesis include various concentric cylinder geometries, a parallel disk geometry and a hybrid planar-cylindrical configuration. Among the range of concentric cylinder cells new designs allow for improved temperature control and the exploration of the influence of curvature which contributes to a spatially dependent shear rate. The parallel disk geometry allows Rheo-NMR practitioners to explore edge effects by either immersing the sample in a sea of fluid or reproducing the experimental conditions of traditional rheometers. Additionally, this device could easily be extended to a cone and plate configuration. The hybrid shear geometry opens up the potential to explore complex fluid behaviour in true simple shear flow and could further be used to study the turbulence of planar Couette flow.

As completed systems the new drive-shaft units discussed in the preceding chapters not only incorporate torque measurements and exciting new shear geometries, but 
introduce new control over the strain input during Rheo-NMR experiments. While oscillatory shear profiles are one result numerous other routines such as stepped flow (increasing or decreasing), shear quench and constant acceleration are now possible. Utilising the digital output and trigger channels on the Bruker spectrometer the drive system developed in this thesis could also be used to study start-up or cessation flow experiments on various fluids.

The design and construction of all the hardware mentioned above has been documented in this thesis; including various chapters in the appendix detailing the layout of printed circuit boards, the assembly of mechanical parts and instructions for interacting with the motor control software. The performance of this hardware was presented with numerous proof of concept validation studies.

Finally, to demonstrate the utility of the hardware and techniques described in this thesis these methods were applied to various open questions in the material science community including exploring the shear-banding phenomenon, the flow of granular materials and shear induced structure transitions.

\subsection{Next Steps for Hardware Development}

Based upon the work of this thesis a short discussion of future hardware development is presented below.

\subsubsection{High-Resolution Rheo-NMR}

Currently the commercially available Rheo-NMR hardware is designed to work solely with micro-imaging resonator coils and gradient systems (e.g. Bruker Micro2.5). Utilising the new drive-shaft design presented in this thesis it would be relatively straightforward to build a shear cell adaptor such that geometries with diameters $<5 \mathrm{~mm}$ could be mounted. As the drive-shaft housing already fits into the spinner housing found on high-resolution NMR magnets the techniques presented in this thesis would be available to practitioners who do not have interest in or access to imaging systems. This work could include high-resolution spectroscopy, multi-nuclear detection or high pulsed field gradient diffusion (e.g. Bruker Diff60). Furthermore, a narrow bore $(<5 \mathrm{~mm})$ shear device could be applied to bench-top permanent magnet NMR spectrometers (e.g. the Magritek SpinSolve) allowing Rheo-NMR measurements to be made without the large footprint and operating costs of spectrometers with a superconducting magnet. 


\subsubsection{Stress Controlled Rheo-NMR}

To date, a stress controlled Rheo-NMR device has not been developed. As strain controlled and stress controlled rheometers each have their own benefits it would be useful to incorporate a stress controlled instrument into the Rheo-NMR toolbox. By imposing a stress (e.g. torque) on a sample under investigation in a rotational shear device a bulk strain (or strain rate) measurement could easily be made with a high resolution optical encoder while local strain rates could be measured via NMR velocimetry techniques. Practically, a stress controlled or EC driven rheometer could be modified and integrated into the NMR environment. However, this approach may be costly and alignment (with negligible friction) of the drive-shaft between the rheometer head above the NMR magnet and the shear cell within the resonator would require particular attention. An alternative would be to place the torque generator inside the bore of the NMR magnet. This would most likely preclude the use of the conventional drag-cup motor or EC motor. One option could be to use a concentric cylinder cell filled with a Newtonian fluid to convert an applied shear rate (from a motor outside of the magnet) to a torque output [190] generated within the bore of a superconducting NMR magnet. A second option could be an air turbine similar to early stress controlled rheometers [36]. Designing this pneumatic system out of NMR compatible materials may allow the drive system to be placed extremely close to the RF coil and minimise the distance between stress generation and the shear device.

\subsubsection{Drive-Shaft Unit for Pressure Driven Shear and Extensional Rheology}

Work in this thesis focused on drag flow shear rheology, however, various aspects of this work could be extended to either pressure driven shear or extensional rheology. In particular, the newly developed control software, electronics and drive system could be used in combination with a lead screw and piston for pressure driven flow (similar to the extensional drive-shaft concept previously introduced [111] and discussed in Section 4.4.3). This design could be combined with a variety of flow cells. Among these, straight pipe geometries could be used to closely model process conditions for materials such as polymer melts. Similar experimental set-ups have been used in past NMR experiments by utilising tubing or pipes and external pumps, however these loop configurations typically require large volumes of sample and can make it difficult to accurately control temperature. Developing this proposed system (with a properly designed backlash free drive screw) would allow oscillatory pipe flow experiments [e.g. 191] to be conducted on a closed and small volume of sample. Alternative geometries to a straight pipe section could include constrictions to reproduce specific process conditions (e.g. step constriction), analogues to porous materials (e.g. numerous parallel 
narrow capillaries or bead packs) or semi-hyperbolic converging dies to produce extensional flow.

\subsection{Final Remarks}

In recent years, while attending and presenting the work in this thesis at conferences and workshops, fellow researchers have frequently commented that instead of building custom hardware it would be better to modify an existing rheometer for use in an NMR system. At face value it is a fair suggestion and seems like a simple approach to achieve a high degree of rheometry resolution. In fact Anton Paar makes the job even easier through the "DSR 502 Measuring Head" instrument [192] which is essentially the motor/transducer and control elements of a rheometer and eliminates the need to dissect a standard bench-top instrument. However, this approach would come at considerable cost; first, the up front cost of the donor rheometer, but also the time and effort to adapt the technology for an NMR magnet. Similarly a bench-top stress controlled instrument (or EC driven instrument), where the motion and measurements are made on the same geometry, is the natural choice to be adapted to the purpose of RheoNMR; however, these instruments are constructed such that the distance between the motor/transducer and shear geometries is on the order of $100 \mathrm{~mm}$. In the case of a superconducting magnet this distance would need to be increased ten fold. Furthermore, the air bearing which is fundamental to the design of the modern rheometer would need to be incorporated into the NMR drive shaft and most certainly within the range of strong magnetic fields (i.e. within the room temperature bore). The work presented in this thesis is not necessarily an alternative to these approaches, but instead is an intermediate step which seeks to generate the interest, capital and enthusiasm to drive the further development of simultaneous high fidelity NMR and rheometry instruments.

The hardware and methods of this thesis have already begun to excite the research community and create a demand for such instruments. Further applications, discussions and collaborations will help to identify what key features are necessary for future developments. Regardless of how these devices continue to evolve, new methods are now available to experimentalists and open up numerous interesting avenues for soft matter research. 


\section{References}

[1] C. W. Macosko, Rheology: Principles, Measurements, and Applications. Wiley-VCH, 1994.

[2] The Society of Rheology, "Official symbols and nomenclature of The Society of Rheology," Journal of Rheology, vol. 57, no. 4, pp. 1047-1055, 2013.

[3] G. W. S. Blair, Elementary Rheology. Academic Press, 1969.

[4] W. R. Schowalter, Mechanics of Non-Newtonian Fluids. Pergamon Press, 1978.

[5] R. I. Tanner, Engineering Rheology. Oxford University Press, 2nd ed., 2000.

[6] H. Barnes, J. F. Hutton, and K. Walters, An Introduction to Rheology. Elsevier, 1989.

[7] R. G. Larson, The Structure and Rheology of Complex Fluids. Oxford University Press, 1999.

[8] L. E. Malvern, Introduction to the Mechanics of a Continuous Medium. Prentice-Hall, 1969.

[9] T. Kambe, Elementary Fluid Mechanics. World Scientific Publishing Co., 2007.

[10] M. Mooney, "A theory of large elastic deformation," Journal of Applied Physics, vol. 11, no. 9, pp. 582-592, 1940.

[11] R. S. Rivlin, "Large elastic deformations of isotropic materials. I. Fundamental concepts," Philosophical Transactions of the Royal Society of London A: Mathematical, Physical and Engineering Sciences, vol. 240, no. 822, pp. 459-490, 1948.

[12] D. T. Kinkbeiner II, Introduction to Matrices and Linear Transformations. W. H. Freeman and Company, 1960.

[13] R. W. Ogden, Non-Linear Elastic Deformations. Dover Publications, Inc., 1984.

[14] M. Reiner, Deformation, Strain and Flow: An Elementary Introduction to Rheology. H.K. Lewis, 1960. 
[15] M. Reiner, "A mathematical theory of dilatancy," American Journal of Mathematics, vol. 67 , no. 3, pp. 350-362, 1945.

[16] R. Rivlin, "The hydrodynamics of non-newtonian fluids. I," Proceedings of the Royal Society of London, Series A (Mathematical and Physical Sciences), vol. 193, pp. 260-281, 1948.

[17] A. W. Sisko, "The flow of lubricating greases," Industrial \& Engineering Chemistry, vol. 50, no. 12, pp. 1789-1792, 1958.

[18] M. M. Cross, "Rheology of non-Newtonian fluids: A new flow equation for pseudoplastic systems," Journal of Colloid Science, vol. 20, no. 5, pp. 417-\&, 1965.

[19] P. J. Carreau, "Rheological equations from molecular network theories," Transactions Of The Society Of Rheology, vol. 16, no. 1, pp. 99-\&, 1972.

[20] E. C. Bingham, "An investigation of the laws of plastic flow," U.S. Bureau of Standards Bulletin, vol. 13, pp. 309-353, 1916.

[21] W. H. Herschel and R. Bulkley, "Konsistenzmessungen von gummibenzollösungen," Kolloid-Zeitschrift, vol. 39, no. 4, pp. 291-300, 1926.

[22] H. Barnes and K. Walters, "The yield stress myth?," Rheologica Acta, vol. 24, no. 4, pp. 323-326, 1985.

[23] H. A. Barnes, "The yield stress-a review or ' $\pi \alpha \nu \tau \alpha \rho \epsilon \iota$ '-everything flows?," Journal of Non-Newtonian Fluid Mechanics, vol. 81, no. 1-2, pp. 133 - 178, 1999.

[24] P. Coussot, "Yield stress fluid flows: A review of experimental data," Journal of Non-Newtonian Fluid Mechanics, vol. 211, pp. 31 - 49, 2014.

[25] W. Weber, “Ueber die elasticität der seidenfäden," Annalen der Physik, vol. 110, no. 2, pp. 247-257, 1835.

[26] M. Reiner, "The Deborah number," Physics Today, vol. 17, no. 1, p. 62, 1964.

[27] J. D. Ferry, Viscoelastic Properties of Polymers. John Wiley \& Sons, 1980.

[28] A. C. Pipkin, Lectures on Viscoelasticity Theory. Springer-Verlag, 1972.

[29] O. E. Meyer, "Theorie der elastischen nachwirkung," Annalen der Physik, vol. 227, no. 1, pp. 108-119, 1874.

[30] J. C. Maxwell, "On the dynamical theory of gases," Philosophical Transactions of the Royal Society of London, vol. 157, pp. 49-88, 1867. 
[31] L. Boltzmann, "Zur theorie der elastischen nachwirkung," Ann. Phys. Chem. Erg., vol. 7, p. $624,1876$.

[32] K. Weissenberg, "A continuum theory of rheological phenomena," Nature, vol. 159, no. 4035, pp. 310-311, 1947.

[33] M. M. Couette, "Etudes sur le frottement des liquides," Annales de chimie et de physique, vol. 21, pp. 433-510, 1890.

[34] H. A. Barnes and D. Bell, "Controlled-stress rotational rheometry: An historical review," Korea-Australia Rheology Journal, vol. 15, pp. 187-196, DEC 2003.

[35] H. A. Barnes, H. Schimanski, and D. Bell, "30 years of progress in viscometers and rheometers," Applied Rheology, vol. 9, no. 2, pp. 69-76, 1999.

[36] S. S. Davis, J. J. Deer, and B. Warburton, "A concentric cylinder air turbine viscometer," Journal of Physics E: Scientific Instruments, vol. 1, no. 9, p. 933, 1968.

[37] G. F. C. Searle, "A simple viscometer for very viscous liquids," Proceedings of the Cambridge Philosophical Society, vol. 16, p. 600, 1912.

[38] G. I. Taylor, "Stability of a viscous liquid contained between two rotating cylinders," Philos Trans R Soc London, Ser A, vol. 223, no. 605-615, pp. 289-343, 1923.

[39] H. Barnes, "A review of the slip (wall depletion) of polymer-solutions, emulsions and particle suspensions in viscometers - its cause, character, and cure," Journal of Non-Newtonian Fluid Mechanics, vol. 56, pp. 221-251, MAR 1995.

[40] A. Yoshimura and R. Prud'homme, "Wall slip corrections for Couette and parallel disk viscometers," Journal of Rheology, vol. 32, pp. 53-67, Jan. 1988.

[41] M. A. Fardin, T. J. Ober, C. Gay, G. Grégoire, G. H. McKinley, and S. Lerouge, “Criterion for purely elastic taylor-couette instability in the flows of shear-banding fluids," EPL (Europhysics Letters), vol. 96, no. 4, p. 44004, 2011.

[42] R. G. Larson, E. S. G. Shaqfeh, and S. J. Muller, "A purely elastic instability in taylor-couette flow," Journal of Fluid Mechanics, vol. 218, pp. 573-600, 91990.

[43] M. Mooney and R. H. Ewart, "The conicylindrical viscometer," Journal of Applied Physics, vol. 5, no. 11, pp. 350-354, 1934.

[44] M. Mooney, "Shearing disc plastometer for unvulcanised rubber," Industrial and Engineering Chemistry (Analytical Edition), vol. 6, pp. 147-151, 1934. 
[45] E. M. Purcell, H. C. Torrey, and R. V. Pound, "Resonance absorption by nuclear magnetic moments in a solid," Phys. Rev., vol. 69, pp. 37-38, Jan 1946.

[46] F. Bloch, W. W. Hansen, and M. Packard, “Nuclear induction," Phys. Rev., vol. 69, pp. 127-127, Feb 1946.

[47] P. T. Callaghan, Principles of Nuclear Magnetic Resonance Microscopy. Oxford University Press, 1991.

[48] M. H. Levitt, Spin Dynamics. Wiley, 2001.

[49] S. Stapf and S.-I. Han, eds., NMR Imaging in Chemical Engineering. Wiley-VCH, 2006.

[50] S. L. Codd and J. D. Seymour, eds., Magnetic Resonance Microscopy: Spatially Resolved NMR Techniques and Applications. Wiley-VCH, 2009.

[51] P. T. Callaghan, Translational Dynamics \& Magnetic Resonance: Principles of Pulsed Gradient Spin Echo NMR. Oxford University Press, 2011.

[52] D. J. Griffiths, Introduction to Quantum Mechanics. Pearson Prentice Hall, 2nd ed., 2005.

[53] A. Abragam, Principles of Nuclear Magnetism. Oxford University Press, 1961.

[54] F. Bloch, “Nuclear induction," Phys. Rev., vol. 70, pp. 460-474, Oct 1946.

[55] E. L. Hahn, “Spin echoes," Phys. Rev., vol. 80, pp. 580-594, Nov 1950.

[56] D. I. Hoult and R. E. Richards, "Signal-to-noise ratio of nuclear magneticresonance experiment," Journal of Magnetic Resonance, vol. 24, no. 1, pp. 71-85, 1976.

[57] G. Bodenhausen, H. Kogler, and R. R. Ernst, "Selection of coherence-transfer pathways in NMR pulse experiments," Journal of Magnetic Resonance, vol. 58, no. 3, pp. 370-388, 1984.

[58] W. G. Proctor and F. C. Yu, "The dependence of a nuclear magnetic resonance frequency upon chemical compound," Phys. Rev., vol. 77, pp. 717-717, Mar 1950.

[59] J. Seelig and W. Niederberger, "Deuterium-labeled lipids as structural probes in liquid crystalline bilayers. A Deuterium magnetic resonance study," Journal of the American Chemical Society, vol. 96, no. 7, pp. 2069-2072, 1974. 
[60] A. Haase, J. Frahm, D. Matthaei, W. Hanicke, and K.-D. Merboldt, “FLASH imaging. Rapid NMR imaging using low flip-angle pulses," Journal of Magnetic Resonance, vol. 67, no. 2, pp. $258-266,1986$.

[61] N. Bloembergen, E. Purcell, and R. Pound, "Relaxation effects in nuclear magnetic resonance absorption," Physical Review, vol. 73, no. 7, pp. 679-712, 1948.

[62] H. Y. Carr and E. M. Purcell, "Effects of diffusion on free precession in nuclear magnetic resonance experiments," Phys. Rev., vol. 94, pp. 630-638, May 1954.

[63] S. Meiboom and D. Gill, "Modified spin-echo method for measuring nuclear magnetic rexalation times," Review of Scientific Instruments, vol. 29, no. 8, pp. 688691, 1958.

[64] P. C. Lauterbur, "Image formation by induced local interactions - examples employing nuclear magnetic-resonance," Nature, vol. 242, no. 5394, pp. 190-191, 1973.

[65] B. Blümich, NMR Imaging of Materials. Oxford University Press, 2000.

[66] P. Mansfield and P. K. Grannell, “NMR ‘Diffraction' In Solids?," Journal Of Physics C-Solid State Physics, vol. 6, no. 22, pp. L422-L426, 1973.

[67] E. Hahn, "Detection of sea-water motion by nuclear precession," Journal Of Geophysical Research, vol. 65, no. 2, pp. 776-777, 1960.

[68] J. Kärger and W. Heink, "The propagator representation of molecular-transport in microporous crystallites," Journal Of Magnetic Resonance, vol. 51, no. 1, pp. 1-7, 1983.

[69] P. T. Callaghan, C. D. Eccles, and Y. Xia, "NMR microscopy of dynamic displacements: k-space and q-space imaging," Journal Of Physics E - Scientific Instruments, vol. 21, pp. 820-822, AUG 1988.

[70] E. Fukushima and S. B. Roeder, Experimental Pulse NMR. Addison-Wesley, 1981.

[71] J. Mispelter, M. Lupa, and A. Briguet, NMR Probeheads: For Biophysical and Biomedical Experiments. Imperial College Press, 2006.

[72] A. Nakatani, M. Poliks, and E. Samulski, "NMR investigation of chain deformation in sheared polymer fluids," Macromolecules, vol. 23, pp. 2686-2692, MAY 14 1990. 
[73] L. Goncalves, J. Casquilho, J. Figueirinhas, C. Cruz, and A. Martins, "Nmr-study of the long-time, defect-controlled, magnetic reorientation of a nematic polymer liquid-crystal," Liquid Crystals, vol. 14, pp. 1485-1493, MAY 1993.

[74] A. Martins, P. Esnault, and F. Volino, "Measurement of the viscoelastic coefficients of main-chain nematic polymers by an NMR technique," Physical Review Letters, vol. 57, pp. 1745-1748, OCT 61986.

[75] M. Britton, P. Callaghan, M. Kilfoil, R. Mair, and K. Owens, "Nmr velocimetry and spectroscopy at microscopic resolution in small rheometric devices," $A P$ PLIED MAGNETIC RESONANCE, vol. 15, no. 3-4, pp. 287-301, 1998. Joint 29th AMPERE / 13th ISMAR International Conference on Magnetic Resonance and Related Phenomena, BERLIN, GERMANY, AUG 02-07, 1998.

[76] R. L. Powell, J. E. Maneval, J. D. Seymour, K. L. McCarthy, and M. J. McCarthy, "Nuclear-magnetic-resonance imaging for viscosity measurements," Journal of Rheology, vol. 38, pp. 1465-1470, SEP-OCT 1994.

[77] R. Mair and P. Callaghan, "Shear flow of wormlike micelles in pipe and cylindrical couette geometries as studied by nuclear magnetic resonance microscopy," $J$ Rheol, vol. 41, pp. 901-924, JUL-AUG 1997.

[78] K. L. McCarthy and W. L. Kerr, "Rheological characterization of a model suspension during pipe flow using MRI," Journal of Food Engineering, vol. 37, pp. 11-23, JUN 1998.

[79] W. B. Yoon and K. L. McCarthy, "Rheology of yogurt during pipe flow as characterized by magnetic resonance imaging," Journal of Texture Studies, vol. 33, pp. 431-444, NOV 2002.

[80] P. Galvosas and P. Callaghan, "Fast magnetic resonance imaging and velocimetry for liquids under high flow rates," J Magn Reson, vol. 181, pp. 119-125, JUL 2006.

[81] J. S. Raynaud, P. Moucheront, J. C. Baudez, F. Bertrand, J. P. Guilbaud, and P. Coussot, "Direct determination by nuclear magnetic resonance of the thixotropic and yielding behavior of suspensions," Journal of Rheology, vol. 46, no. 3, pp. 709-732, 2002.

[82] J. Keegan, D. Firmin, P. Gatehouse, and D. Longmore, "Velocity mapping of coronary artery blood flow," Magnetic Resonance Materials In Physics Biology And Medicine, vol. 2, pp. 311-314, OCT 1994. 1st Nottingham Symposium on Magnetic Resonance Imaging, NOTTINGHAM, ENGLAND, 1994. 
[83] P. Callaghan, "Rheo-NMR: nuclear magnetic resonance and the rheology of complex fluids," Reports On Progress In Physics, vol. 62, pp. 599-670, APR 1999.

[84] P. Callaghan, "Rheo-NMR: A new window on the rheology of complex fluids," in Encyclopedia of Nuclear Magnetic Resonance (D. M. Grant and R. K. Harris, eds.), vol. 9, pp. 737-750, John Wiley \& Sons, 2002.

[85] A. B. Tayler, D. J. Holland, A. J. Sederman, and L. F. Gladden, "Exploring the origins of turbulence in multiphase flow using compressed sensing MRI," Phys. Rev. Lett., vol. 108, p. 264505, Jun 2012.

[86] S. Kuczera, RARE Velocimetry of Shear Banded Flow in Cylindrical Couette Geometry. $\mathrm{PhD}$ thesis, Victoria University of Wellington, 2015.

[87] S. Kuczera and P. Galvosas, "Advances and artefact suppression in RAREvelocimetry for flow with curved streamlines," Journal of Magnetic Resonance, 2015.

[88] G. E. Pavlovskaya and T. Meersmann, "Spatial mapping of flow-induced molecular alignment in a noncrystalline biopolymer fluid using double quantum filtered (DQF) Na-23 MRI," Journal of Physical Chemistry Letters, vol. 5, pp. 2632-2636, AUG 72014.

[89] P. Callaghan and E. Fischer, "Rheo-NMR: A new application for nmr microscopy and nmr spectroscopy," Bruker Annual Report, pp. 34-38, 2001.

[90] Magritek, Rheo-NMR Manual, 3.1 .1 ed. http://www.rheo-nmr.com/ manuals/rheomanual.pdf.

[91] D. A. Grabowski and C. Schmidt, "Simultaneous measurement of shear viscosity and director orientation of a side-chain liquid-crystalline polymer by RheoNMR," Macromolecules, vol. 27, pp. 2632-2634, APR 251994.

[92] M. Badiger, P. Rajamohanan, P. Suryavanshi, S. Ganapathy, and R. Mashelkar, "In situ rheo-NMR investigations of shear-dependent $\mathrm{H}-1$ spin relaxation in polymer solutions," Macromolecules, vol. 35, pp. 126-134, JAN 12002.

[93] H. Siebert, D. A. Grabowski, and C. Schmidt, "Rheo-nmr study of a non-flowaligning side-chain liquid crystal polymer in nematic solution," Rheologica Acta, vol. 36, no. 6, pp. 618-627, 1997.

[94] U. Böhme and U. Scheler, An NMR Investigation of a Polymer Melt under Shear, ch. 28, pp. 431-438. Oxford University Press, 2011. 
[95] R. Muthupillai, D. Lomas, P. Rossman, J. Greenleaf, A. Manduca, and R. Ehman, "Magnetic-resonance elastography by direct visualization of propagating acoustic strain waves," Science, vol. 269, pp. 1854-1857, SEP 291995.

[96] Y. K. Mariappan, K. J. Glaser, and R. L. Ehman, “Magnetic resonance elastography: A review," Clinical Anatomy, vol. 23, pp. 497-511, JUL 2010.

[97] S. F. Othman, H. Xu, T. J. Royston, and R. L. Magin, "Microscopic magnetic resonance elastography ( $\mu \mathrm{MRE}), "$ Magnetic Resonance in Medicine, vol. 54, no. 3, pp. 605-615, 2005.

[98] G. Eidmann, R. Savelsberg, P. Blumler, and B. Blumich, "The NMR MOUSE, a mobile universal surface explorer," Journal Of Magnetic Resonance Series A, vol. 122, pp. 104-109, SEP 1996.

[99] F. Casanova, J. Perlo, and B. Blümich, "Velocity distributions remotely measured with a single-sided nmr sensor," J Magn Reson, vol. 171, pp. 124-130, NOV 2004.

[100] J. Perlo, F. Casanova, and B. Blümich, “Velocity imaging by ex situ NMR,, J Magn Reson, vol. 173, pp. 254-258, APR 2005.

[101] B. Blumich, S. Anferova, K. Kremer, S. Sharma, V. Herrmann, and A. Segre, "Unilateral nuclear magnetic resonance for quality control: The NMR-MOUSE," Spectroscopy, vol. 18, pp. 18+, FEB 2003.

[102] J. Z. Zhen, High Efficieny Class-D RF Amplifiers and Mobile NMR Systems. PhD thesis, Victoria University of Wellington, 2015.

[103] J. Mitchell, L. F. Gladden, T. C. Chandrasekera, and E. J. Fordham, "Low-field permanent magnets for industrial process and quality control," Progress In Nuclear Magnetic Resonance Spectroscopy, vol. 76, pp. 1-60, JAN 2014.

[104] D. F. Arola, G. A. Barrall, R. L. Powell, K. L. McCarthy, and M. J. McCarthy, “Use of nuclear magnetic resonance imaging as a viscometer for process monitoring," Chemical Engineering Science, vol. 52, no. 13, pp. 2049 - 2057, 1997. Process Tomography.

[105] Aspect Imaging, "Flowscan real-time rheology system and solutions," 2015. http://www. aspectimaging.com/advanced-industrial/product/ flowscan-real-time-rheology-system [Accessed: 29 Aug 2015].

[106] F. Deng, L. Xiao, W. Chen, H. Liu, G. Liao, M. Wang, and Q. Xie, "Rapid determination of fluid viscosity using low-field two-dimensional NMR," Journal of Magnetic Resonance, vol. 247, pp. 1-8, OCT 2014. 
[107] A. Muhammad and R. B. de Vasconcellos Azeredo, “H-1 NMR spectroscopy and low-field relaxometry for predicting viscosity and API gravity of Brazilian crude oils - A comparative study," Fuel, vol. 130, pp. 126-134, AUG 152014.

[108] S. Kahle, W. Nussbaum, M. Hehn, H. P. Raich, M. Wilhelm, and P. Bluemler, "Combination of NMR relaxometry and mechanical testing during vulcanization," Kautschuk, Gummi, Kunststoffe, vol. 61, pp. 92-94, MAR 2008.

[109] V. Raentzsch, K.-F. Ratzsch, G. Guthausen, S. Schlabach, and M. Wilhelm, "Molecular dynamics of polymer composites using rheology and combined RheoNMR on the example of $\mathrm{TiO}_{2}$-filled poly(n-alkyl methacrylates) and trans1,4-polyisoprene," Soft Materials, vol. 12, no. 1, SI, pp. S4-S13, 2014.

[110] R. Cormier, C. Schmidt, and P. Callaghan, "Director reorientation of a side-chain liquid crystalline polymer under extensional flow," J Rheol, vol. 48, pp. 881-894, JUL-AUG 2004.

[111] B. Douglass, Rheo-NMR Invesigations of Flow and Alignment in Complex Fluids. PhD thesis, Victoria University of Wellington, 2011.

[112] D. F. James, "Flow in a converging channel at moderate reynolds numbers," AIChE Journal, vol. 37, no. 1, pp. 59-64, 1991.

[113] N. M. Loening, J. Keeler, and G. A. Morris, "One-dimensional DOSY," Journal of Magnetic Resonance, vol. 153, no. 1, pp. 103 -112, 2001.

[114] W. C. Kittler, P. Galvosas, and M. W. Hunter, "Parallel acquisition of q-space using second order magnetic fields for single-shot diffusion measurements," Journal of Magnetic Resonance, vol. 244, pp. 46-52, JUL 2014.

[115] W. C. Kittler, S. Obruchkov, P. Galvosas, and M. W. Hunter, "Pulsed second order field $\mathrm{nmr}$ for real time pgse and single-shot surface to volume ratio measurements," Journal of Magnetic Resonance, vol. 247, pp. 42-49, OCT 2014.

[116] W. Kittler, Pulsed Second Order Magnetic Field Nuclear Magnetic Resonance for RealTime Fluid Transport Measurements. PhD thesis, Victoria University of Wellington, 2014.

[117] W. C. Kittler, P. Galvosas, and M. W. Hunter, "Pulsed second order fields for parallel acquisition of q-space," Microporous and Mesoporous Materials, vol. 205, pp. 61-64, MAR 152015. 
[118] W. C. Kittler, M. W. Hunter, and P. Galvosas, "Real-time fluid transport characterization through direct acquisition of the averaged propagator," Physical Review E, vol. 92, pp. 3016-3016, AUG 2015.

[119] "CRC Handbook of Chemistry and Physics; Internet Version," 2016. http:// www. hbcpnetbase.com/ [Accessed: 21 Nov 2015].

[120] Engineers Edge, LLC, "PolyEtherEtherKetone," $2015 . \quad$ http: //engineersedge.com/plastic/plastic_material/ polyetheretherketone-peek-plastic.htm [Accessed: 21 Nov 2015].

[121] MakeItFrom.com, "Unfilled PEEK," 2015. http://www.makeitfrom.com/ material-properties/Unfilled-PEEK/ [Accessed: 21 Nov 2015].

[122] MakeItFrom.com, “Borosilicate Glass," 2015. http: / / www . makeit from. com/ material-properties/Borosilicate-Glass/ [Accessed: 21 Nov 2015].

[123] Corning Incorporated, "MACOR ${ }^{\circledR}$ Machinable Glass," 2015. https: //www.corning.com/worldwide/en/products/advanced-optics/ product-materials/specialty-glass-and-glass-ceramics/ glass-ceramics/macor.html [Accessed: 21 Nov 2015].

[124] N. Tillmark and P. H. Alfredsson, "Experiments on transition in plane couette flow," J Fluid Mech, vol. 235, pp. 89-102, 11993.

[125] D. Krug, B. Lüthi, H. Seybold, M. Holzner, and A. Tsinober, "3D-PTV measurements in a plane couette flow," Experiments in Fluids, vol. 52, pp. 1349-1360, 2012.

[126] J. J. Kobine and T. Mullin, "Nonlinear phenomena in hybrid couette flow composed of planar and circular shear," Phys Fluids, vol. 13, no. 6, pp. 1583-1593, 2001.

[127] EVA Robotics Pty Ltd, EvoDrive ST-23 Integration Manual, FW-UM009 Rev C ed., December 2013. http: / /www . evarobo. com/support.

[128] EVA Robotics Pty Ltd, EvoDrive Programmers Manual, FW-UM001 Rev D ed., September 2012. http: / / www . evarobo. com/support.

[129] T. I. Brox and P. Galvosas, "Rheological measurement devices," Mar. 5 2015. WO Patent App. PCT/NZ2014/000,167.

[130] T. I. Brox and P. Galvosas, "Rheological measurement device with torque sensor," Mar. 5 2015. WO Patent App. PCT/NZ2014/000,168. 
[131] Vibrac, "Torque Transducers," 2015. http://vibrac.com/torque_ transducers . php [Accessed: 24 Nov 2015].

[132] C. W. De Silva, Mechatronics: A Foundation Course. CRC Press, 2010.

[133] Arduino, 2015. https://www.arduino.cc/.

[134] A. D'Ausilio, "Arduino: A low-cost multipurpose lab equipment," Behavior Research Methods, vol. 44, no. 2, pp. 305-313, 2012.

[135] Arduino, "Arduino Due," 2015. https://www.arduino.cc/en/Main/ ArduinoBoardDue [Accessed: 26 Nov 2015].

[136] Atmel Corporation, "ATSAM3X8E Cortex-M3 MCU," 2015. http://www . atmel. com/devices/SAM3X8E. aspx [Accessed: 26 Nov 2015].

[137] Vibrac, 1312 Interface Card: Specifications and User Information. also see http: //vibrac.com/transducers_model_1312.php.

[138] "socat - Multipurpose relay," 2015. http://www.dest-unreach.org/ socat / [Accessed: 26 Nov 2015].

[139] EVA Robotics Pty Ltd, EvoDuino Programmers Manual, FW-UM011 Rev A ed., November 2011. http: / / www. evarobo. com/support.

[140] T. I. Brox, "rheoControl Firmware," 2015. http://oscar.ecs.vuw.ac.nz/ tb/motionControl/firmware/rheoControl4Due/rheoControl4Due. ino.

[141] Python Software Foundation, "Python," 2015. https: / / www . python . org/.

[142] "PySide," 2015. https://wiki.qt.io/PySide.

[143] "PySide 1.2.1 Reference," 2015. http: / / pyside.github.io/docs/pyside/ [Accessed: 26 Nov 2015].

[144] S. Kuczera, C. Perge, M.-A. Fardin, T. I. Brox, M. A. K. Williams, S. Manneville, and P. Galvosas, "Anomalous shear banding revisited with Rheo-NMR and RheoUSV," Rheologica Acta, vol. 54, pp. 619-636, JUL 2015.

[145] COMSOL, "COMSOL Multiphysics Modelling Software," 2015. http: //www . $\mathrm{comsol} . \mathrm{com} /$.

[146] B. Munro, T. I. Brox, P. Galvosas, R. Dykstra, E. Harjes, P. J. B. Edwards, and M. A. K. Williams, "Rheo-NMR studies of the effects of the reorientation of 
protein-containing nematic bacteriophage phases on measured residual diploar couplings," in preparation, 2015.

[147] T. I. Brox, B. Douglass, J. R. Brown, and P. Galvosas, "Observations of the influence of couette cell curvature on shear banding in surfactant wormlike micelles," in review Journal of Rheology, 2015.

[148] S. Lerouge and J. Berret, "Shear-induced transitions and instabilities in surfactant wormlike micelles," arXiv, pp. 1-71, 2010.

[149] H. Rehage and H. Hoffmann, "Viscoelastic surfactant solutions - model systems for rheological research," Molecular Physics, vol. 74, pp. 933-973, DEC 101991.

[150] M. Cates, T. McLeish, and G. Marrucci, "The rheology of entangled polymers at very high shear rates," Europhys Lett, vol. 21, pp. 451-456, FEB 11993.

[151] R. Mair and P. Callaghan, "Observation of shear banding in worm-like micelles by NMR velocity imaging," Europhys Lett, vol. 36, pp. 719-724, DEC 201996.

[152] Y. Hu and A. Lips, "Kinetics and mechanism of shear banding in an entangled micellar solution," Journal of Rheology, vol. 49, pp. 1001-1027, SEP-OCT 2005.

[153] J. B. Salmon, S. Manneville, and A. Colin, "Shear banding in a lyotropic lamellar phase. I. Time-averaged velocity profiles," Physical Review E, vol. 68, no. 5, p. 051503, 2003.

[154] V. Herle, S. Manneville, and P. Fischer, "Ultrasound velocimetry in a shearthickening wormlike micellar solution: Evidence for the coexistence of radial and vorticity shear bands," European Physical Journal E, vol. 26, no. 1, pp. 3-12, 2008.

[155] S. M. Fielding, "Complex dynamics of shear banded flows," Soft Matter, vol. 3, no. 10, pp. 1262-1279, 2007.

[156] M.-A. Fardin and S. Lerouge, "Instabilities in wormlike micelle systems," The European Physical Journal E, vol. 35, no. 9, 2012.

[157] M. A. Fardin, T. Divoux, M. A. Guedeau-Boudeville, I. Buchet-Maulien, J. Browaeys, G. H. McKinley, S. Manneville, and S. Lerouge, "Shear-banding in surfactant wormlike micelles: elastic instabilities and wall slip," Soft Matter, vol. 8, pp. 2535-2553, 2012.

[158] M. Lopez-Gonzalez, W. Holmes, P. Callaghan, and P. Photinos, "Shear banding fluctuations and nematic order in wormlike micelles," Phys Rev Lett, vol. 93, DEC 312004. 
[159] M. R. Lopez-Gonzalez, W. M. Holmes, and P. T. Callaghan, "Rheo-NMR phenomena of wormlike micelles," Soft Matter, vol. 2, pp. 855-869, OCT 72006.

[160] B. S. Douglass, R. H. Colby, L. A. Madsen, and P. T. Callaghan, "Rheo-NMR of wormlike micelles formed from nonionic pluronic surfactants," Macromolecules, vol. 41, pp. 804-814, FEB 122008.

[161] B. Medronho, J. Brown, M. G. Miguel, C. Schmidt, U. Olsson, and P. Galvosas, "Planar lamellae and onions: a spatially resolved rheo-NMR approach to the shear-induced structural transformations in a surfactant model system," Soft Matter, vol. 7, no. 10, pp. 4938-4947, 2011.

[162] P. Callaghan and A. Gil, "Rheo-NMR of semidilute polyacrylamide in water," Macromolecules, vol. 33, pp. 4116-4124, MAY 302000.

[163] P. T. Callaghan, "Rheo NMR and shear banding," Rheologica Acta, vol. 47, pp. 243255, APR 2008

[164] H. Wassenius and P. Callaghan, "NMR velocimetry studies of the steady-shear rheology of a concentrated hard-sphere colloidal system," Eur Phys J E, vol. 18, pp. 69-84, SEP 2005.

[165] A. Lutti and P. T. Callaghan, "Measurement of multilamellar onion dimensions under shear using frequency domain pulsed gradient NMR," J Magn Reson, vol. 187, pp. 251-257, AUG 2007.

[166] W. Holmes, P. Callaghan, D. Vlassopoulos, and J. Roovers, "Shear banding phenomena in ultrasoft colloidal glasses," J Rheol, vol. 48, pp. 1085-1102, SEP-OCT 2004.

[167] M. Britton and P. Callaghan, "Nuclear magnetic resonance visualization of anomalous flow in cone-and-plate rheometry," J Rheol, vol. 41, pp. 1365-1386, NOV-DEC 1997.

[168] S. M. Fielding and H. J. Wilson, "Shear banding and interfacial instability in planar poiseuille flow," J Non-Newtonian Fluid Mech, vol. 165, pp. 196-202, MAR 2010.

[169] K. W. Feindel and P. T. Callaghan, "Anomalous shear banding: Multidimensional dynamics under fluctuating slip conditions," Rheol Acta, vol. 49, pp. 1003-1013, OCT 2010. 
[170] A. J. Sederman, M. D. Mantle, C. Buckley, and L. F. Gladden, “MRI technique for measurement of velocity vectors, acceleration, and autocorrelation functions in turbulent flow," Journal of Magnetic Resonance, vol. 166, pp. 182-189, FEB 2004.

[171] C. J. Davies, A. J. Sederman, C. J. Pipe, G. H. McKinley, L. F. Gladden, and M. L. Johns, "Rapid measurement of transient velocity evolution using gervais," J Magn Reson, vol. 202, pp. 93-101, JAN 2010.

[172] A. Tayler, A. Sederman, B. Newling, M. Mantle, and L. Gladden, “'Snap-shot' velocity vector mapping using echo-planar imaging," Journal of Magnetic Resonance, vol. 204, no. 2, pp. 266-272, 2010.

[173] M. P. Lettinga and S. Manneville, "Competition between shear banding and wall slip in wormlike micelles," Phys Rev Lett, vol. 103, DEC 112009.

[174] H. T. Fabich, T. I. Brox, J. R. Brown, S. L. Codd, P. Galvosas, A. J. Sederman, J. D. Seymour, and D. J. Holland, "Spatially resolved measurements of the variance in velocity for granular flow using a Rheo-NMR device," in preparation, 2015.

[175] H. M. Jaeger, S. R. Nagel, and R. P. Behringer, "Granular solids, liquids, and gases," Reviews of Modern Physics, vol. 68, pp. 1259-1273, Oct 1996.

[176] P. Richard, M. Nicodemi, R. Delannay, P. Ribiere, and D. Bideau, "Slow relaxation and compaction of granular systems," Nature Materials, vol. 4, pp. 121-128, FEB 2005.

[177] C. S. Campbell, "Granular material flows - an overview," Powder Technology, vol. 162, no. 3, pp. $208-229,2006$.

[178] P. Jop, Y. Forterre, and O. Pouliquen, "A constitutive law for dense granular flows," Nature, vol. 441, pp. 727-730, JUN 82006.

[179] C. K. K. Lun, S. B. Savage, D. J. Jeffrey, and N. Chepurniy, "Kinetic theories for granular flow - inelastic particles in couette-flow and slightly inelastic particles in a general flowfield," Journal Of Fluid Mechanics, vol. 140, no. MAR, pp. 223-256, 1984.

[180] J. D. Seymour, A. Caprihan, S. A. Altobelli, and E. Fukushima, "Pulsed gradient spin echo nuclear magnetic resonance imaging of diffusion in granular flow," Physical Review Letters, vol. 84, pp. 266-269, JAN 102000.

[181] S. Harms, S. Stapf, and B. Blumich, "Application of k- and q-space encoding NMR techniques on granular media in a 3D model fluidized bed reactor," Journal of Magnetic Resonance, vol. 178, pp. 308-317, FEB 2006. 
[182] D. J. Holland, C. R. Mueller, J. S. Dennis, L. F. Gladden, and A. J. Sedennan, “Spatially resolved measurement of anisotropic granular temperature in gas-fluidized beds," Powder Technology, vol. 182, pp. 171-181, FEB 222008.

[183] P. Moucheront, F. Bertrand, G. Koval, L. Tocquer, S. Rodts, J.-N. Roux, A. Corfdir, and F. Chevoir, "MRI investigation of granular interface rheology using a new cylinder shear apparatus," Magnetic Resonance Imaging, vol. 28, pp. 910-918, JUL 2010.

[184] B. Medronho, M. G. Miguel, and U. Olsson, "Viscoelasticity of a nonionic lamellar phase," Langmuir, vol. 23, no. 10, pp. 5270-5274, 2007. PMID: 17439163.

[185] B. Medronho, S. Shafaei, R. Szopko, M. G. Miguel, U. Olsson, and C. Schmidt, "Shear-induced transitions between a planar lamellar phase and multilamellar vesicles: Continuous versus discontinuous transformation," Langmuir, vol. 24, no. 13, pp. 6480-6486, 2008. PMID: 18517228.

[186] B. Medronho, C. Schmidt, U. Olsson, and M. G. Miguel, "Size determination of shear-induced multilamellar vesicles by Rheo-NMR spectroscopy," Langmuir, vol. 26, no. 3, pp. 1477-1481, 2010. PMID: 20099913.

[187] B. Medronho, M. Rodrigues, M. G. Miguel, U. Olsson, and C. Schmidt, "Shearinduced defect formation in a nonionic lamellar phase," Langmuir, vol. 26, no. 13, pp. 11304-11313, 2010.

[188] C. Oliviero, L. Coppola, R. Gianferri, I. Nicotera, and U. Olsson, "Dynamic phase diagram and onion formation in the system $\mathrm{C}_{10} \mathrm{E}_{3} / \mathrm{D}_{2} \mathrm{O}$," Colloids and Surfaces $A$ : Physicochemical and Engineering Aspects, vol. 228, pp. 85-90, NOV 12003.

[189] S. Kuczera, L. Gentile, T. I. Brox, U. Olsson, C. Schmidt, and P. Galvosas, "Large amplitude oscillatory shear of $\mathrm{C}_{10} \mathrm{E}_{3}$," in preparation, 2015.

[190] B. van den Brule and S. Kadijk, "A simple constant-stress rheometer," Journal of Non-Newtonian Fluid Mechanics, vol. 43, no. 1, pp. 127 - 139, 1992.

[191] L. Q. Evertz, E. M. Rassi, J. R. M. Kennedy, S. L. Codd, and J. D. Seymour, “Oscillatory flow phenomena in simple and complex fluids," Applied Magnetic Resonance, vol. 42, pp. 211-225, MAR 2012.

[192] Anton Paar, "Rheometer Measuring Head," 2015. http: //www . anton-paar . $\mathrm{com} /$ us-en/products/details/rheometer-measuring-head/ rheometer/ [Accessed: 10 Dec 2015]. 


\section{Appendix A}

\section{Equations of Motion for a Viscous}

\section{Fluid}

\section{A.1 Rectangular Coordinates}

$x$-component:

$$
\rho\left(\frac{\partial v_{x}}{\partial t}+v_{x} \frac{\partial v_{x}}{\partial x}+v_{y} \frac{\partial v_{x}}{\partial y}+v_{z} \frac{\partial v_{x}}{\partial z}\right)=-\frac{\partial p}{\partial x}+\left(\frac{\partial \tau_{x x}}{\partial x}+\frac{\partial \tau_{y x}}{\partial y}+\frac{\partial \tau_{z x}}{\partial z}\right)+\rho g_{x}
$$

$y$-component:

$$
\rho\left(\frac{\partial v_{y}}{\partial t}+v_{x} \frac{\partial v_{y}}{\partial x}+v_{y} \frac{\partial v_{y}}{\partial y}+v_{z} \frac{\partial v_{y}}{\partial z}\right)=-\frac{\partial p}{\partial y}+\left(\frac{\partial \tau_{x y}}{\partial x}+\frac{\partial \tau_{y y}}{\partial y}+\frac{\partial \tau_{z y}}{\partial z}\right)+\rho g_{y}
$$

$z$-component:

$$
\rho\left(\frac{\partial v_{z}}{\partial t}+v_{x} \frac{\partial v_{z}}{\partial x}+v_{y} \frac{\partial v_{z}}{\partial y}+v_{z} \frac{\partial v_{z}}{\partial z}\right)=-\frac{\partial p}{\partial z}+\left(\frac{\partial \tau_{x z}}{\partial x}+\frac{\partial \tau_{y z}}{\partial y}+\frac{\partial \tau_{z z}}{\partial z}\right)+\rho g_{z}
$$

\section{A.2 Cylindrical Coordinates}

$r$-component:

$$
\begin{aligned}
\rho\left(\frac{\partial v_{r}}{\partial t}+v_{r} \frac{\partial v_{r}}{\partial r}+\frac{v_{\theta}}{r} \frac{\partial v_{r}}{\partial \theta}-\frac{v_{\theta}^{2}}{r}+v_{z} \frac{\partial v_{r}}{\partial z}\right) & = \\
& \frac{1}{r} \frac{\partial}{\partial r}\left(r \tau_{r r}\right)+\frac{1}{r} \frac{\partial \tau_{\theta r}}{\partial \theta}+\frac{\partial \tau_{z r}}{\partial z}-\frac{\tau_{\theta \theta}}{r}-\frac{\partial p}{\partial r}+\rho g_{r}
\end{aligned}
$$

$\theta$-component:

$$
\begin{aligned}
\rho\left(\frac{\partial v_{\theta}}{\partial t}+v_{r} \frac{\partial v_{\theta}}{\partial r}+\right. & \left.\frac{v_{\theta}}{r} \frac{\partial v_{\theta}}{\partial \theta}+\frac{v_{r} v_{\theta}}{r}+v_{z} \frac{\partial v_{\theta}}{\partial z}\right)= \\
& \frac{1}{r^{2}} \frac{\partial}{\partial r}\left(r^{2} \tau_{r \theta}\right)+\frac{1}{r} \frac{\partial \tau_{\theta \theta}}{\partial \theta}+\frac{\partial \tau_{z \theta}}{\partial z}+\frac{\tau_{\theta r}-\tau_{r \theta}}{r}-\frac{1}{r} \frac{\partial p}{\partial \theta}+\rho g_{\theta}
\end{aligned}
$$


$z$-component:

$$
\begin{aligned}
& \rho\left(\frac{\partial v_{z}}{\partial t}+v_{r} \frac{\partial v_{z}}{\partial r}+\frac{v_{\theta}}{r} \frac{\partial v_{z}}{\partial \theta}+v_{z}\right.\left.\frac{\partial v_{z}}{\partial z}\right)= \\
& \frac{1}{r} \frac{\partial}{\partial r}\left(r \tau_{r z}\right)+\frac{1}{r} \frac{\partial \tau_{\theta z}}{\partial \theta}+\frac{\partial \tau_{z z}}{\partial z}-\frac{\partial p}{\partial z}+\rho g_{z}
\end{aligned}
$$

\section{A.3 Spherical Coordinates}

r-component:

$$
\begin{aligned}
& \rho\left(\frac{\partial v_{r}}{\partial t}+v_{r} \frac{\partial v_{r}}{\partial r}+\frac{v_{\theta}}{r} \frac{\partial v_{r}}{\partial \theta}+\frac{v_{\phi}}{r \sin \theta} \frac{\partial v_{r}}{\partial \phi}-\frac{v_{\theta}^{2}+v_{\phi}^{2}}{r}\right)= \\
& -\frac{\partial p}{\partial r}+\left(\frac{1}{r^{2}} \frac{\partial}{\partial r}\left(r^{2} \tau_{r r}\right)+\frac{1}{r \sin \theta} \frac{\partial}{\partial \theta}\left(\tau_{\theta r} \sin \theta\right)+\frac{1}{r \sin \theta} \frac{\partial \tau_{r \phi}}{\partial \phi}-\frac{\tau_{\theta \theta}+\tau_{\phi \phi}}{r}\right)+p g_{r}
\end{aligned}
$$

$\theta$-component:

$$
\begin{aligned}
& \rho\left(\frac{\partial v_{\theta}}{\partial t}+v_{r} \frac{\partial v_{\theta}}{\partial r}+\frac{v_{\theta}}{r} \frac{\partial v_{\theta}}{\partial \theta}+\frac{v_{\theta}}{r \sin \theta} \frac{\partial v_{\theta}}{\partial \phi}+\frac{v_{r} v_{\theta}}{r}+\frac{v_{\phi}^{2}}{r} \cot \theta\right)=-\frac{1}{r} \frac{\partial p}{\partial \theta}+ \\
& \left(\frac{1}{r^{3}} \frac{\partial}{\partial r}\left(r^{3} \tau_{r \theta}\right)+\frac{1}{r \sin \theta} \frac{\partial}{\partial \theta}\left(\tau_{\theta \theta} \sin \theta\right)+\frac{1}{r \sin \theta} \frac{\partial \tau_{\phi \theta}}{\partial \phi}+\frac{\left(\tau_{\theta r}-\tau_{r \theta}\right)-\cot \theta \tau_{\phi \phi}}{r}\right)+p g_{\theta}
\end{aligned}
$$

$\phi$-component:

$$
\begin{gathered}
\rho\left(\frac{\partial v_{\phi}}{\partial t}+v_{r} \frac{\partial v_{\phi}}{\partial r}+\frac{v_{\theta}}{r} \frac{\partial v_{\phi}}{\partial \theta}+\frac{v_{\phi}}{r \sin \theta} \frac{\partial v_{\phi}}{\partial \phi}+\frac{v_{\phi} v_{r}}{r}+\frac{v_{\theta} v_{\phi}}{r} \cot \theta\right)=-\frac{1}{r \sin \theta} \frac{\partial p}{\partial \phi}+ \\
\left(\frac{1}{r^{3}} \frac{\partial}{\partial r}\left(r^{3} \tau_{r \phi}\right)+\frac{1}{r \sin \theta} \frac{\partial\left(\tau_{\theta \phi} \sin \theta\right)}{\partial \theta}+\frac{1}{r \sin \theta} \frac{\partial \tau_{\phi \phi}}{\partial \phi}+\frac{\left(\tau_{\phi r}-\tau_{r \phi}\right)-2 \cot \theta \tau_{\theta \phi}}{r}\right)+p g_{\phi}
\end{gathered}
$$




\section{Appendix B}

\section{Mechanical Design}

\section{B.1 Shear Geometries}

- Concentric Cylinder

- Bayonet Connection Variant (Figure B.1)

- Zero Backlash Variant (Figure B.2)

- High Resolution Variant (Figure B.3)

- TA Instruments AR 2000 Accessory (Figure B.4)

- Torque Drive-Shaft Variant (Figure B.5)

- Parallel Disks

- Zero Backlash Variant (Figure B.6)

- TA Instruments AR 2000 Accessory (Figure B.7)

- Planar-Cylindrical Hybrid (Figure B.8) 


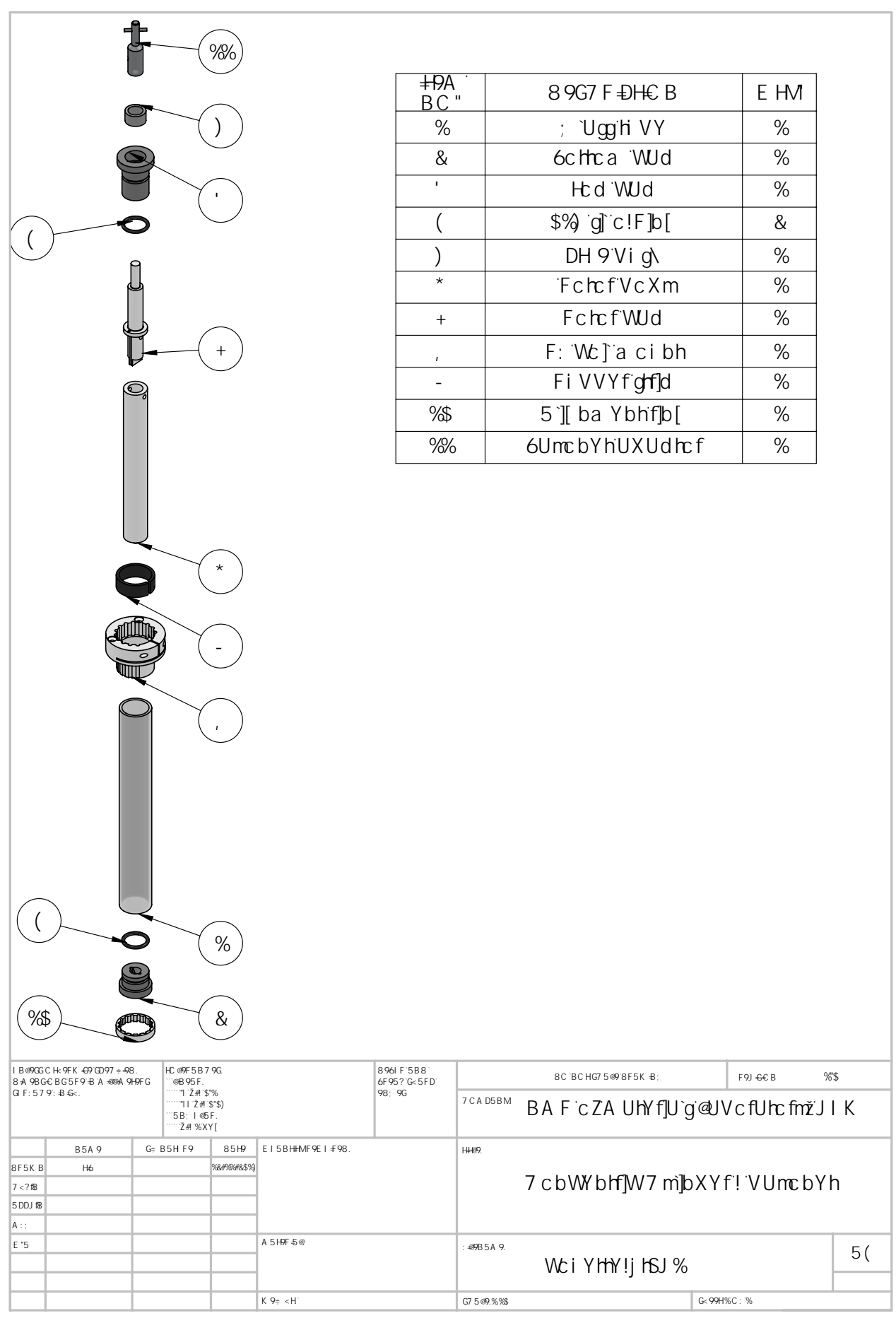

Figure B.1: Exploded view of concentric cylinder geometry with bayonet style connection. 


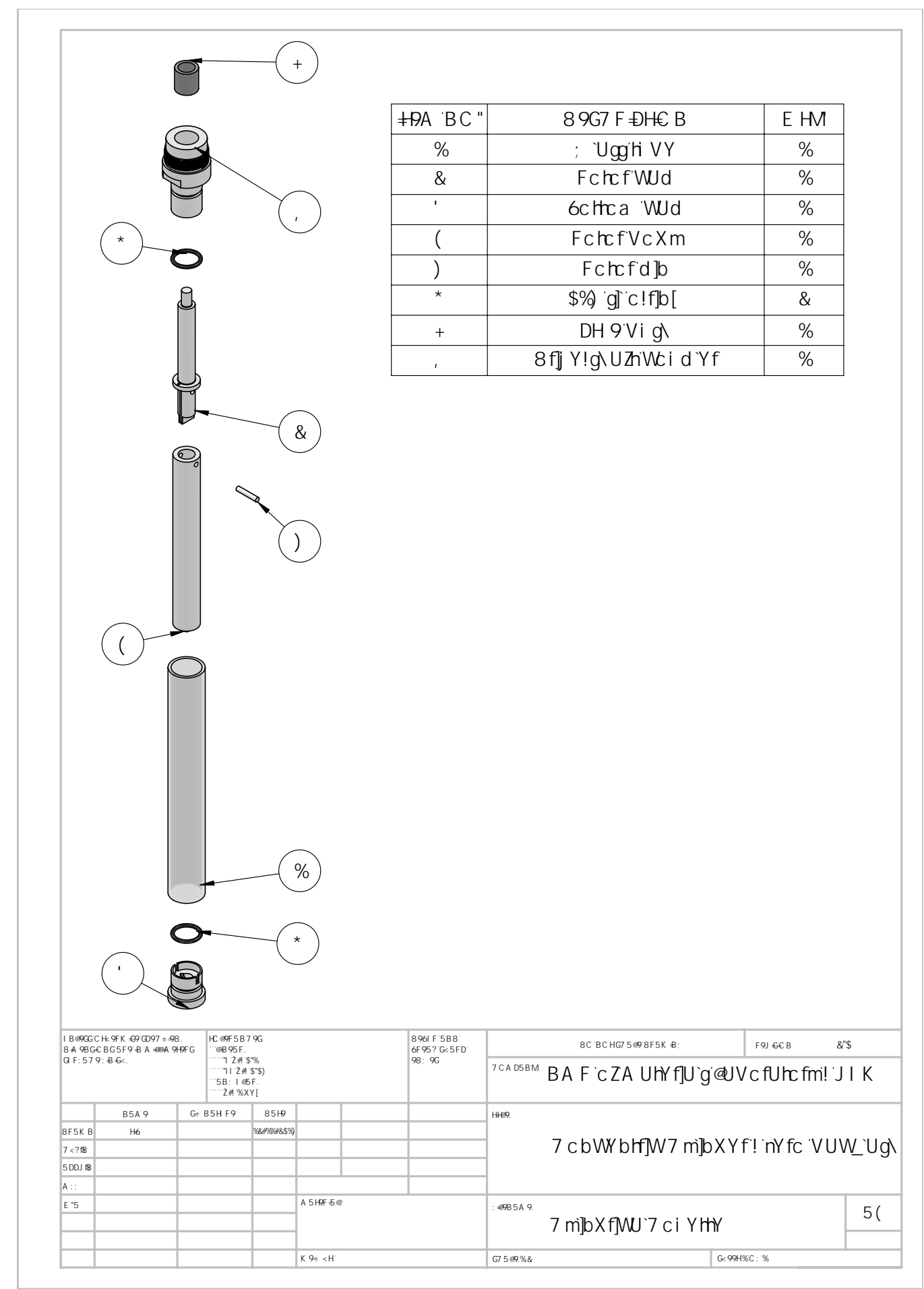

Figure B.2: Exploded view of concentric cylinder geometry which mounts directly to the drive-shaft unit to eliminate backlash. 


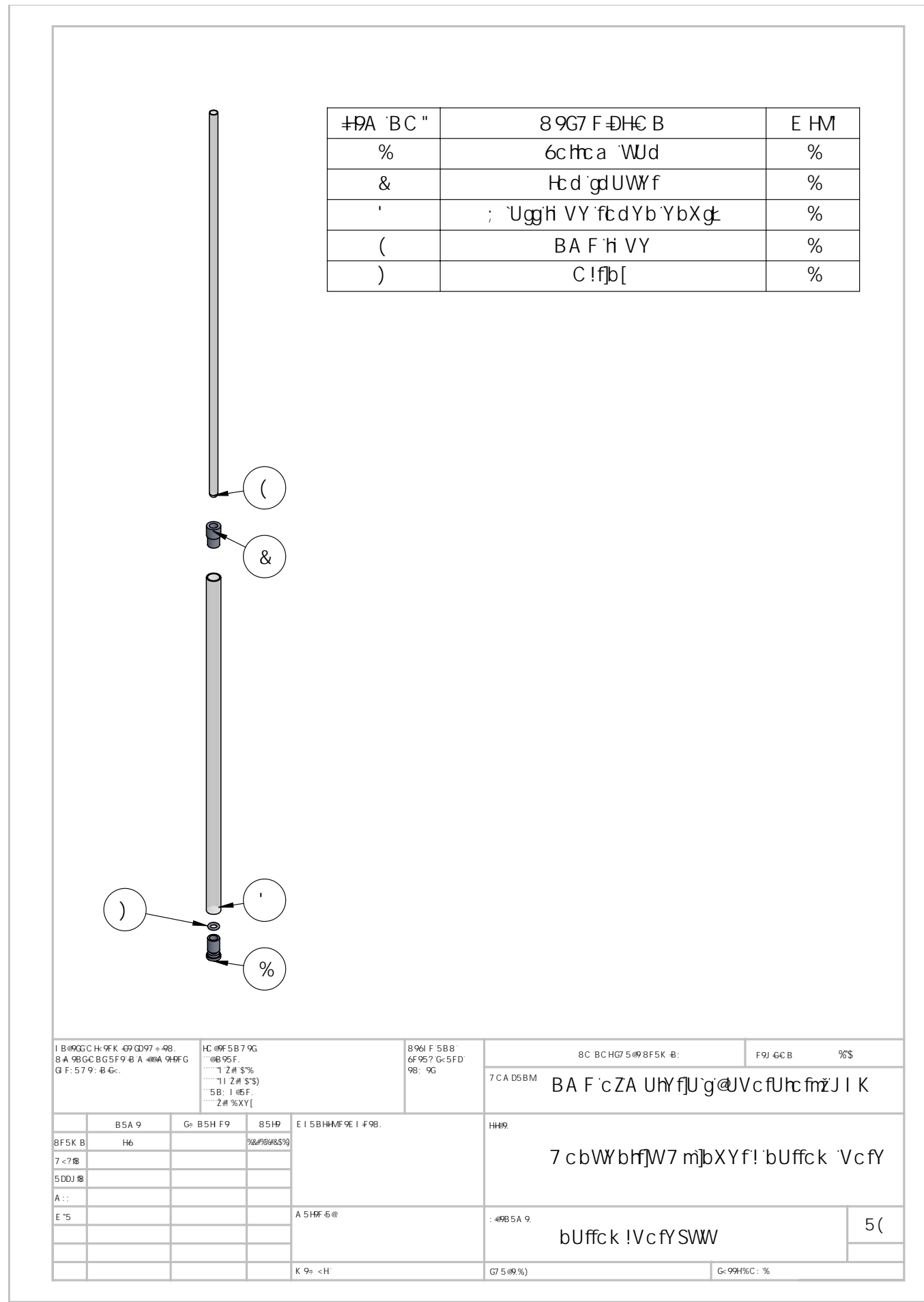

Figure B.3: Exploded view of concentric cylinder $(\mathrm{OD}<5 \mathrm{~mm})$ for high resolution NMR systems. 


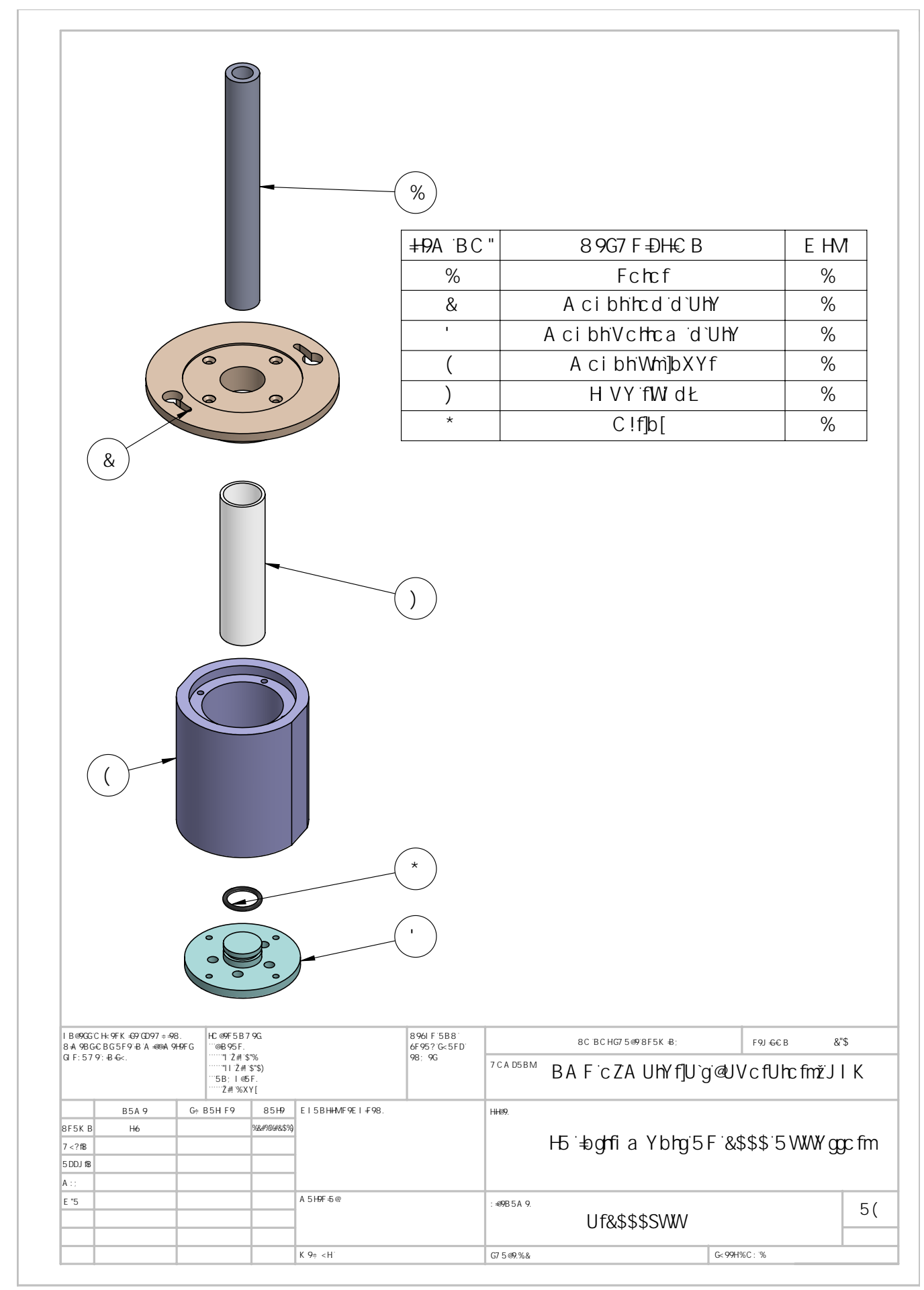

Figure B.4: Exploded view of TA Instrument AR 2000 concentric cylinder accessory. The three part mount piece fits within the peltier jacket accessory available from TA Instruments. 


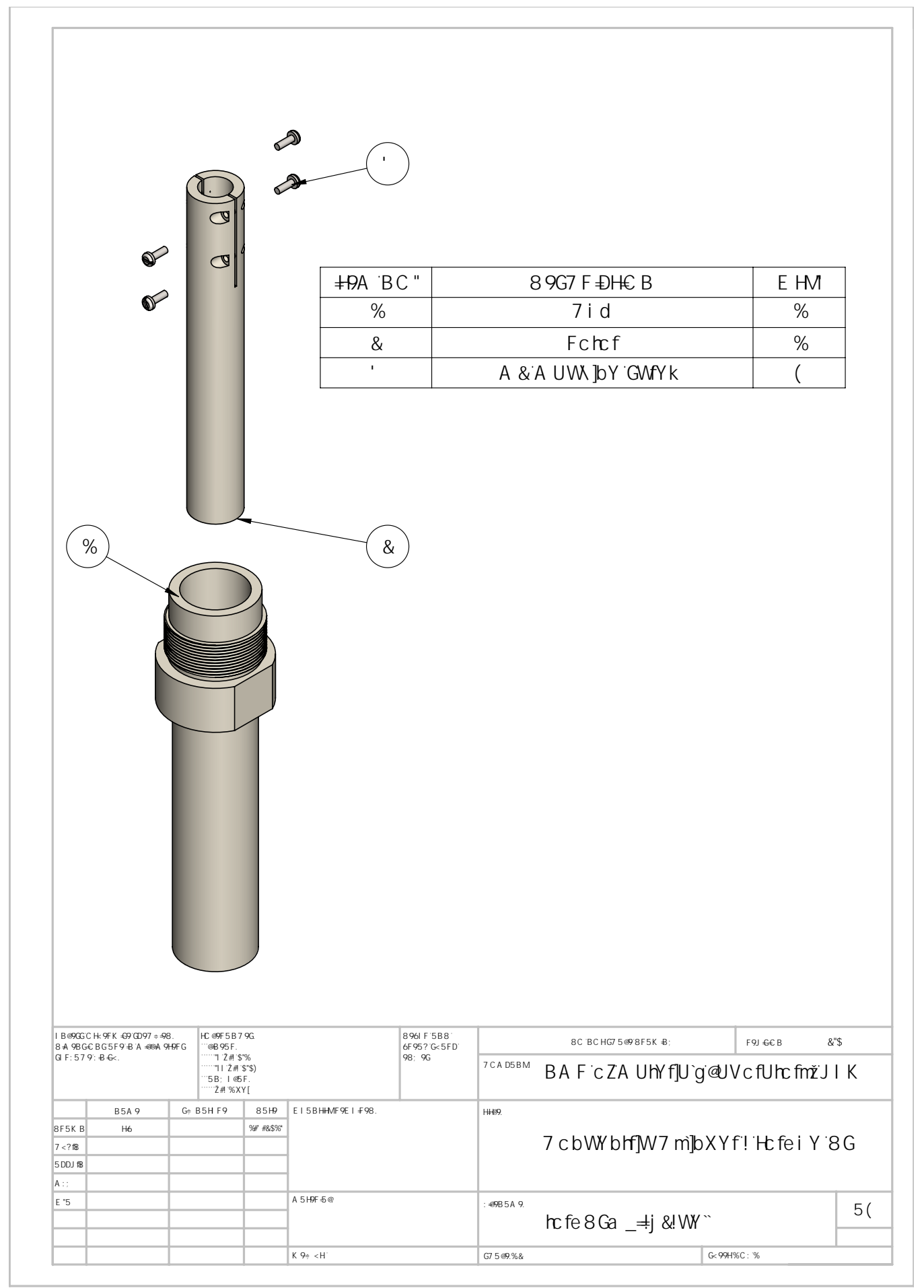

Figure B.5: Exploded view of concentric cylinder geometry for drive-shaft unit with integrated torque transducer. 


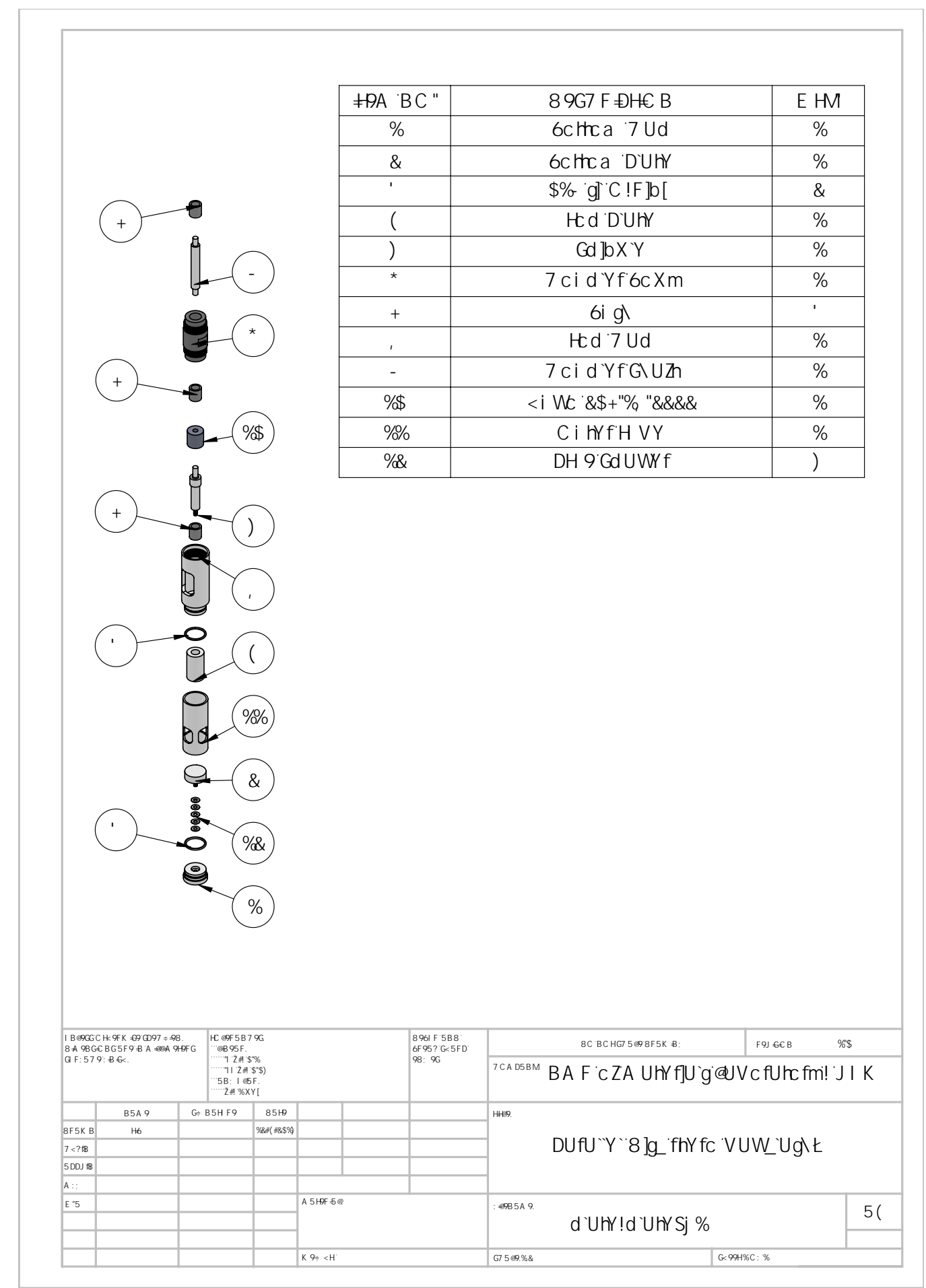

Figure B.6: Exploded view of parallel disks geometry for new drive-shaft unit. 


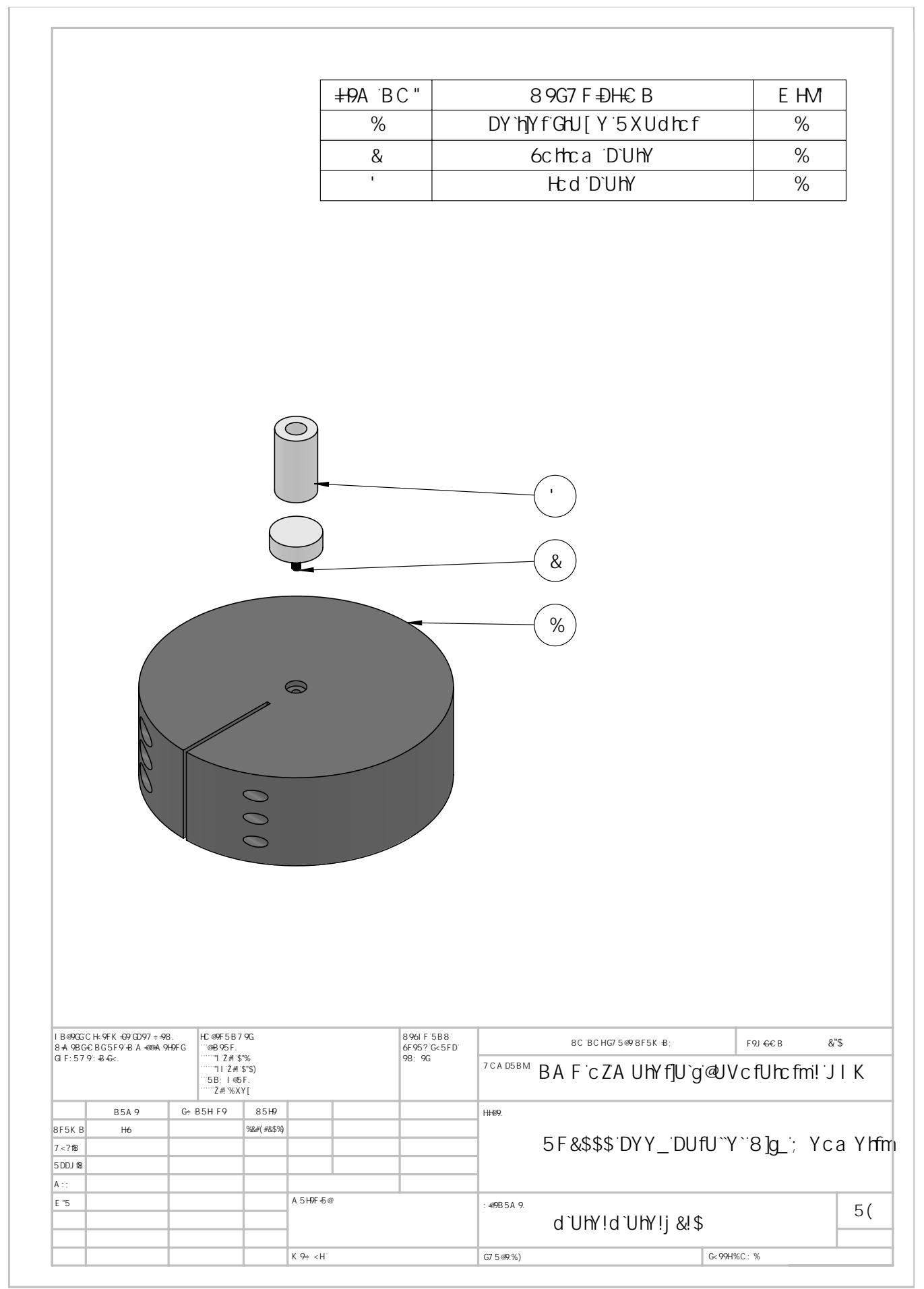

Figure B.7: Exploded view of parallel disks accessory which allows the shear geometries from the NMR shear device (Figure B.6) to be used on a TA Instruments AR 2000 rheometer. 


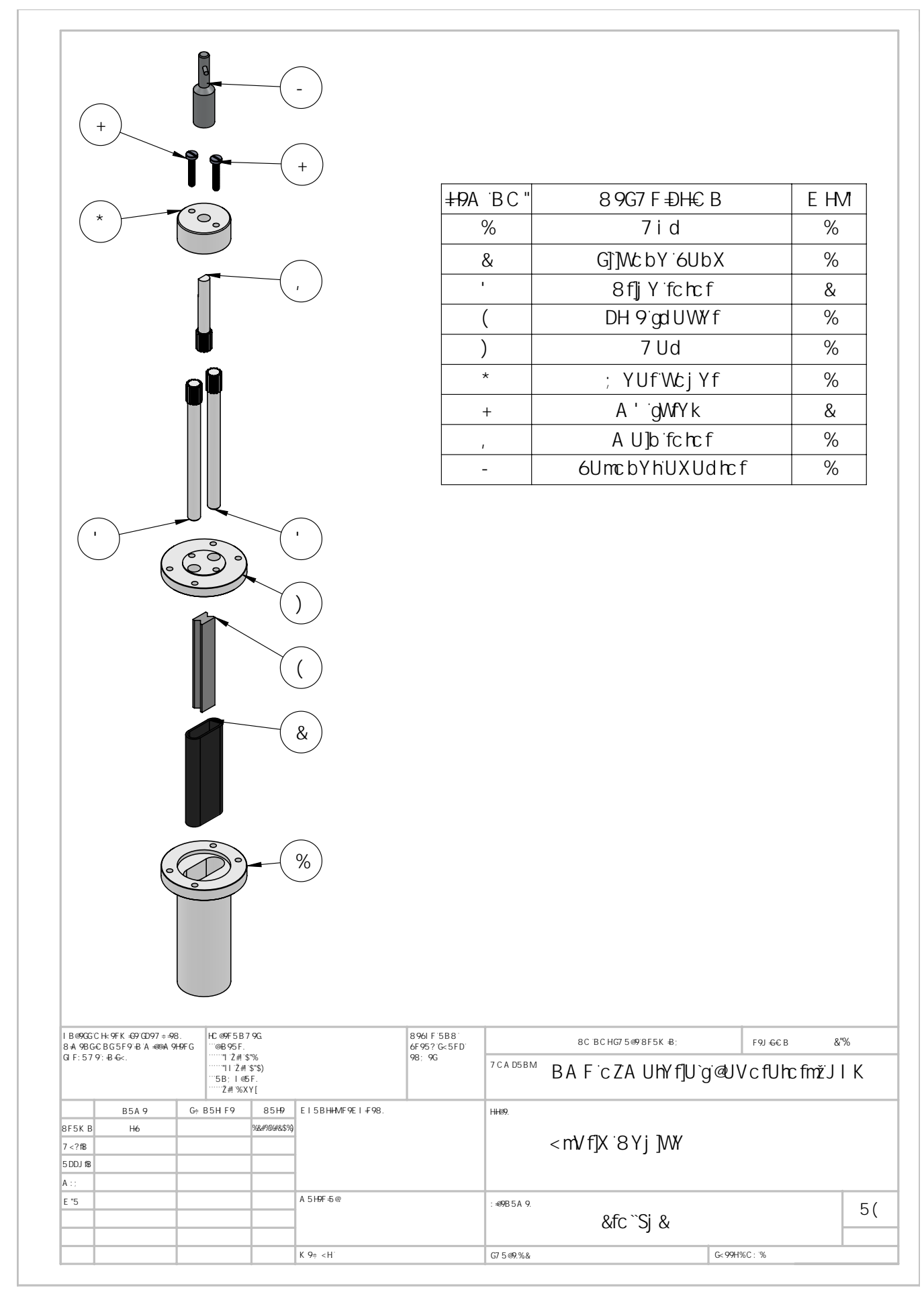

Figure B.8: Exploded view of hybrid shear device. 


\section{B.2 Drive-Shaft Units}

- Generic Design (Figure B.9)

- Torque Sensor Variant (Figure B.10)

- Super Wide Bore Variant (Figure B.11)

- Super Wide Bore Shim Barrel Adaptor (Figure B.12) 


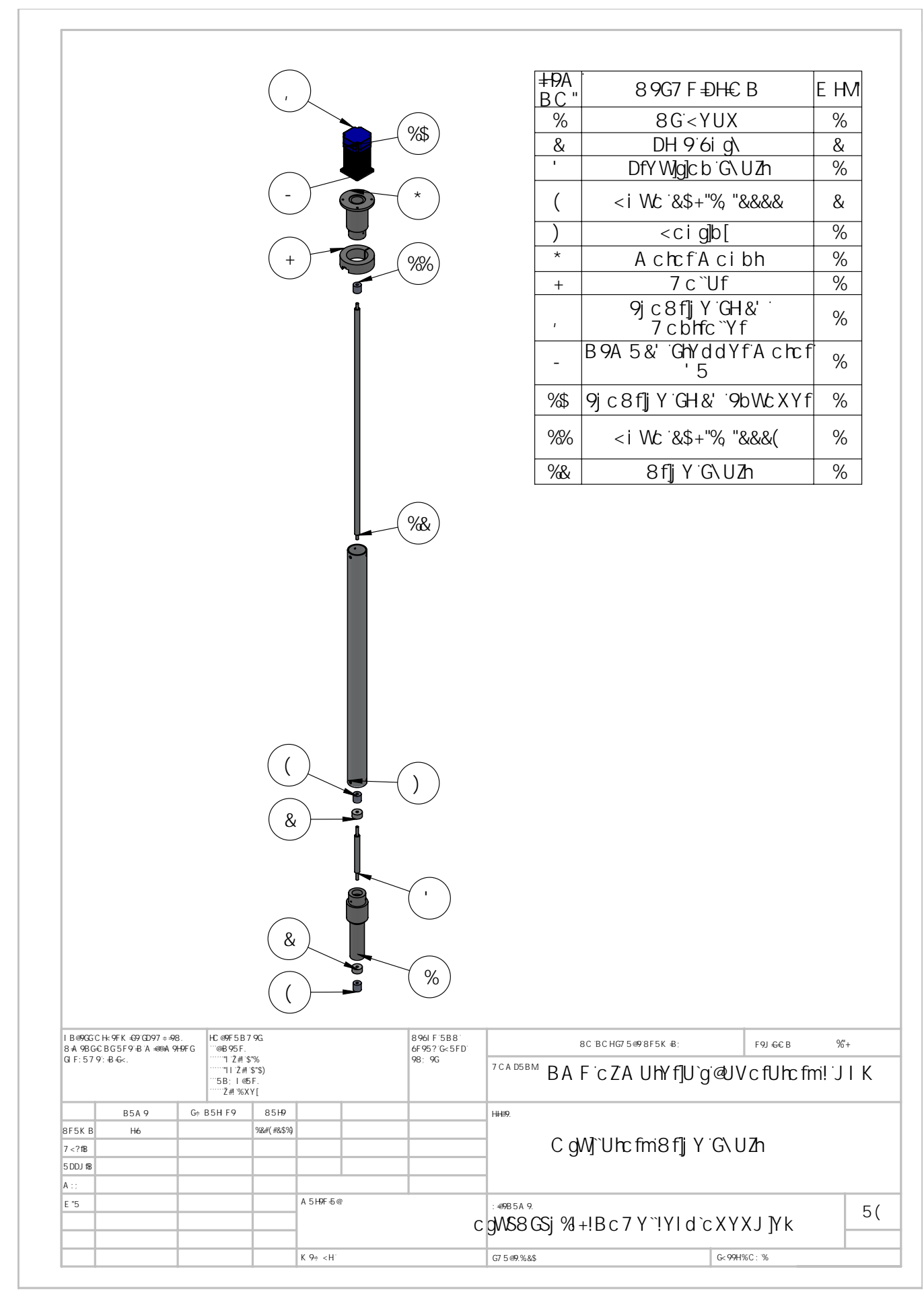

Figure B.9: Exploded view of generic (wide bore) drive-shaft unit. 


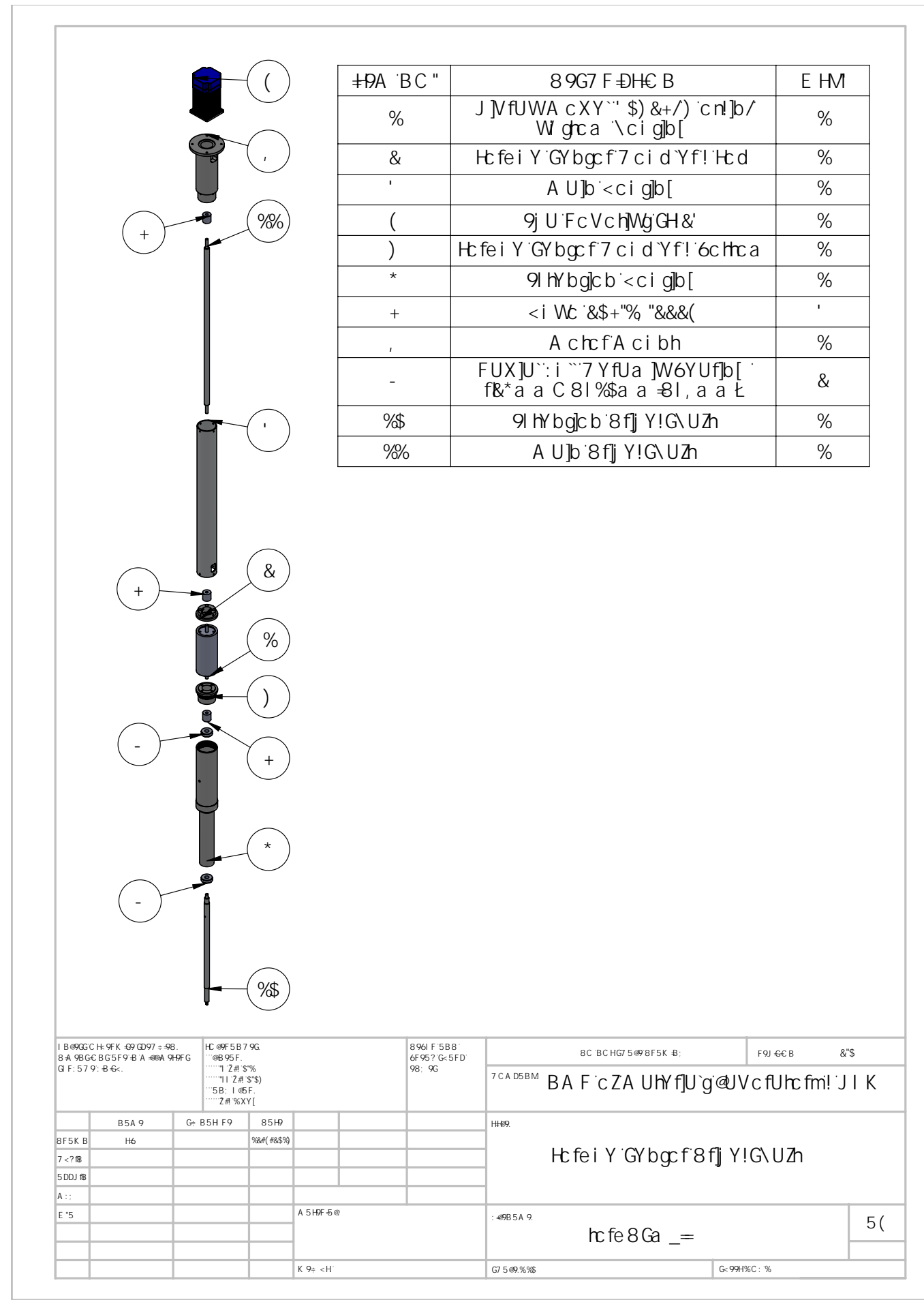

Figure B.10: Exploded view of wide bore drive-shaft unit with integrated torque transducer. 


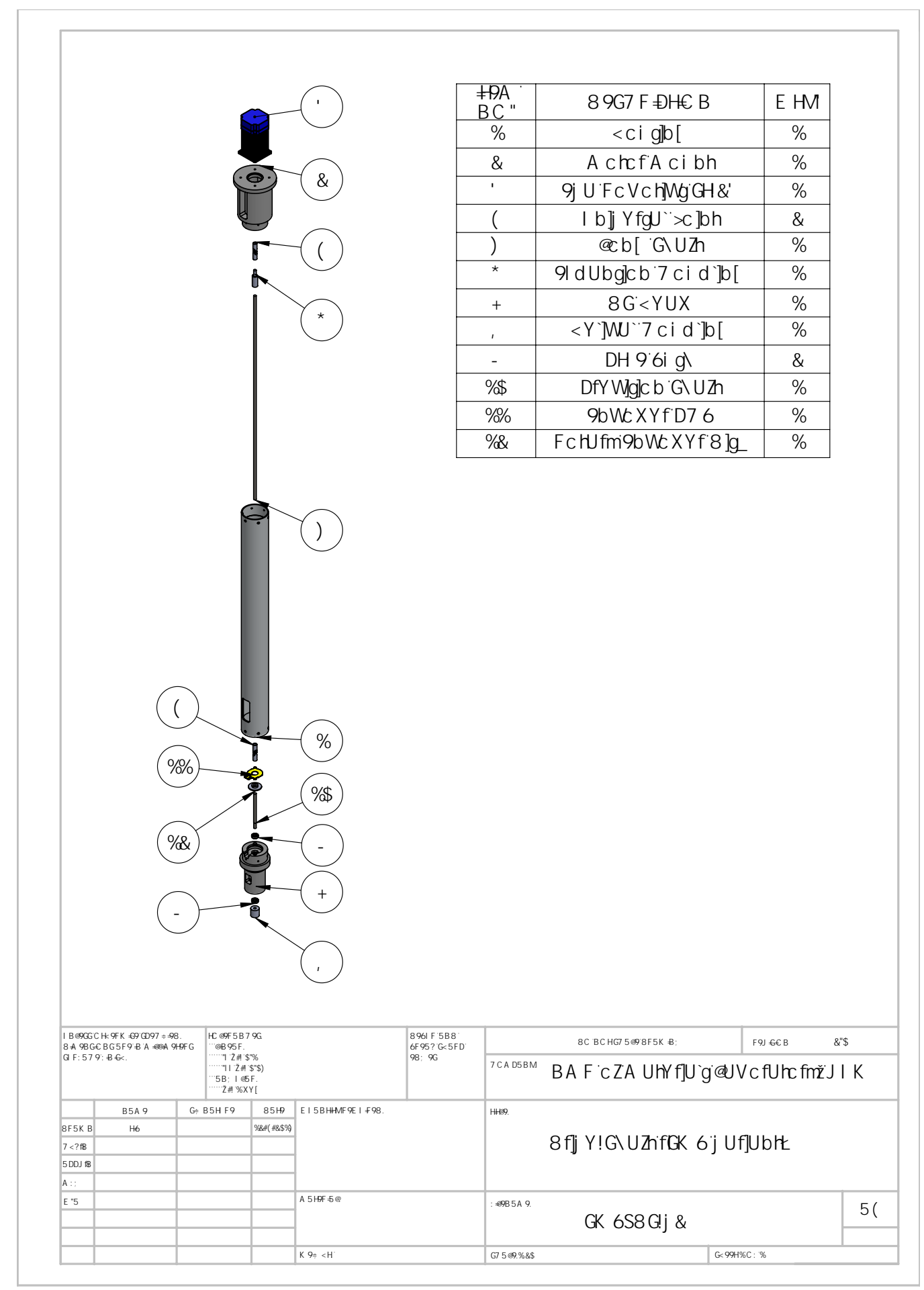

Figure B.11: Exploded view of super wide bore drive-shaft unit. 


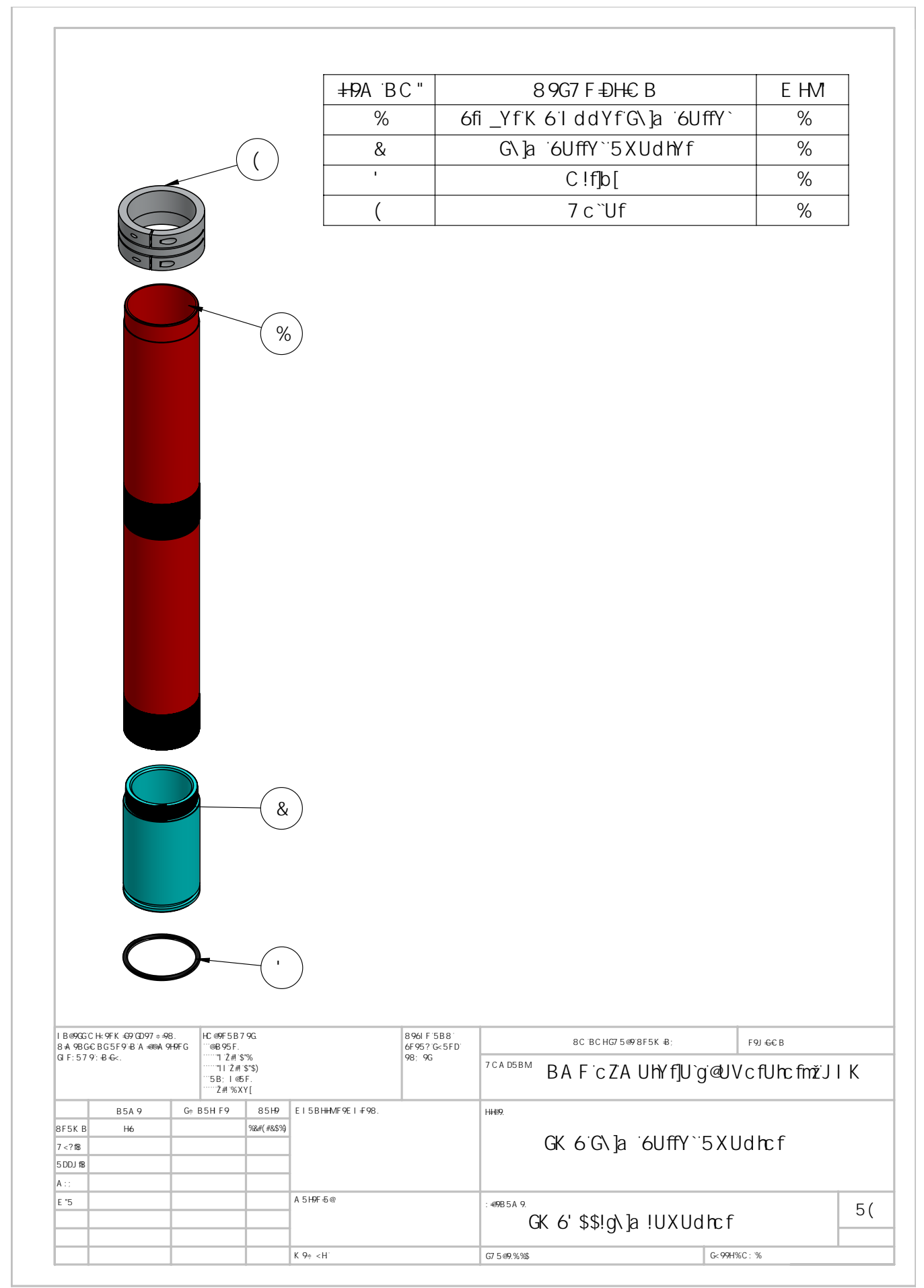

Figure B.12: Exploded view of super wide bore upper shim barrel adaptor for mounting drive-shaft unit within magnet. 


\section{Appendix C}

\section{PCB Design}

\section{C.1 Control Electronics}

\begin{tabular}{lccc} 
Part & Schematic & Component Layout & Bill of Material \\
\hline rheoMotor & Figure C.1 & Figure C.2 & Figure C.3 \\
rheoDueShield & Figure C.4 & Figure C.5 & Figure C.6
\end{tabular}




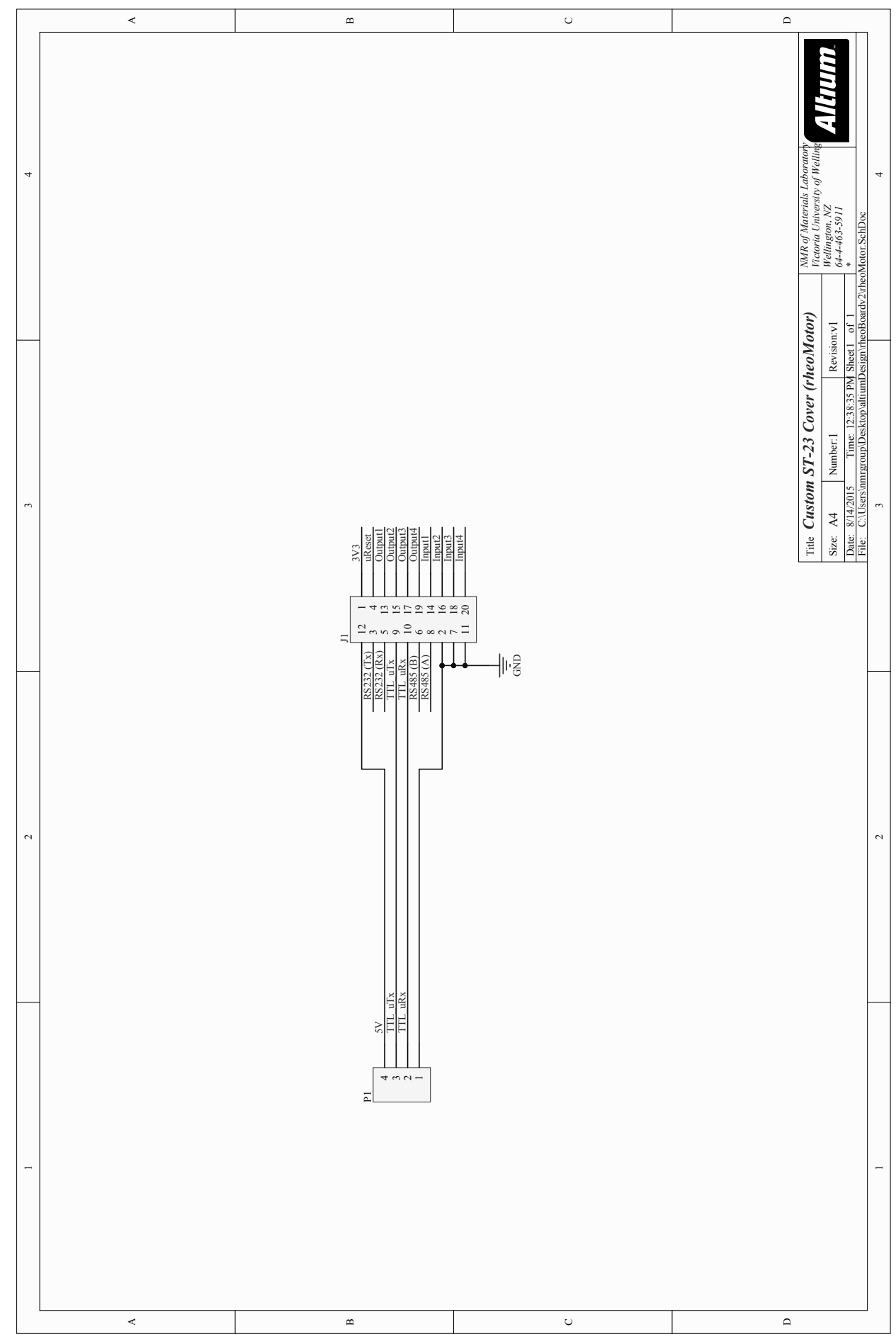

Figure C.1: rheoMotor v1 schematic 


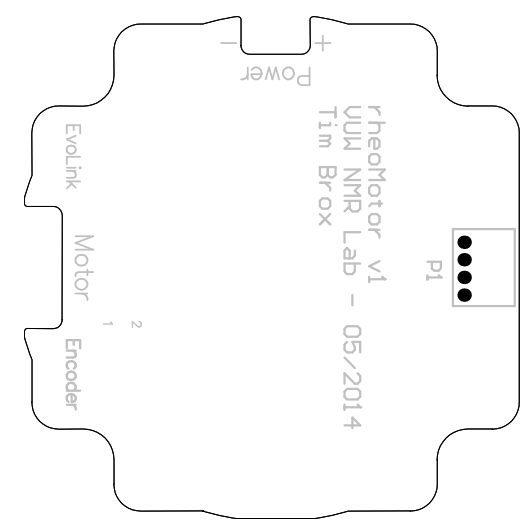

(a) Top

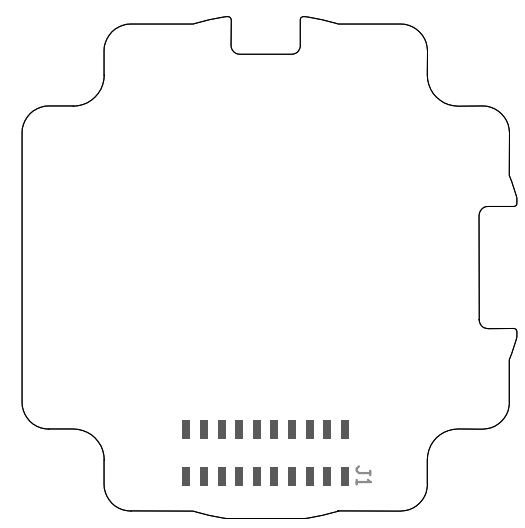

(b) Bottom

Figure C.2: rheoMotor v1 PCB component layout

\begin{tabular}{|c|l|r|l|}
\hline Description & \multicolumn{1}{|c|}{ Designator } & Quantity & \multicolumn{1}{c|}{ Digikey PN } \\
\hline CONN RECEPT 2MM SMD AU 2OPOS & J1 & & 952-1349-5-ND \\
\hline CONN HEADER PH SIDE 4POS 2MM & P1 & & 1 \\
\hline
\end{tabular}

Figure C.3: rheoMotor v1 bill of material 


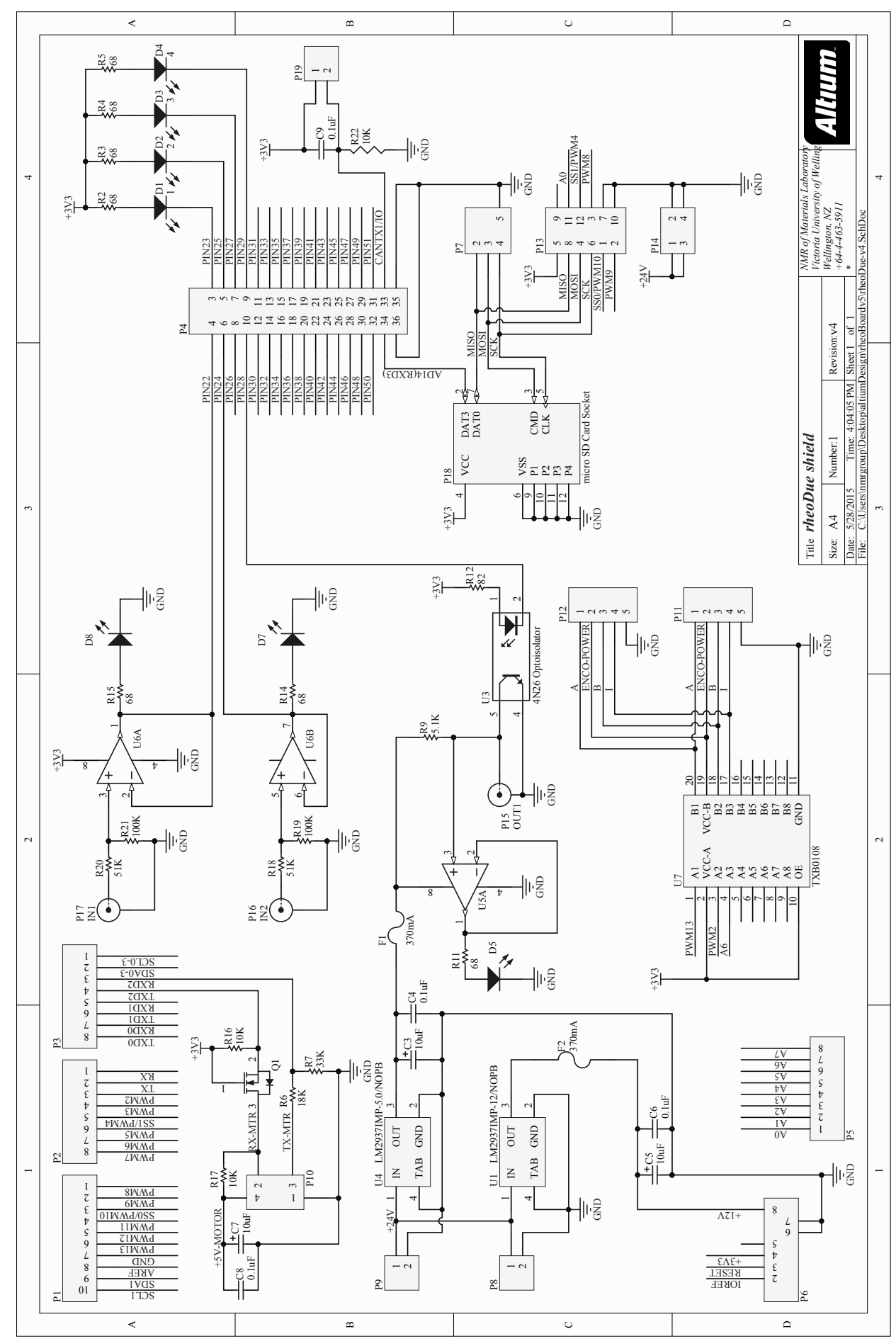

Figure C.4: rheoDueShield v4 schematic 


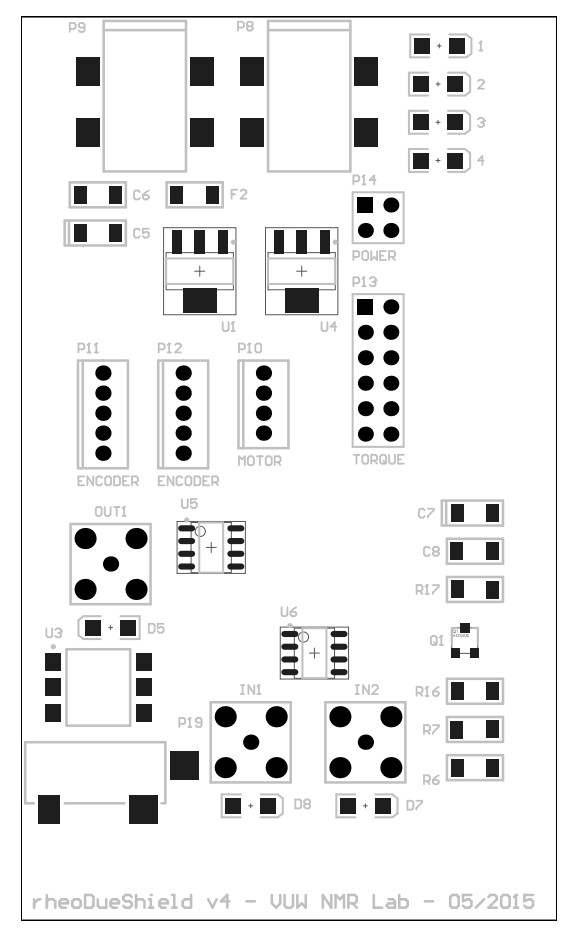

(a) Top

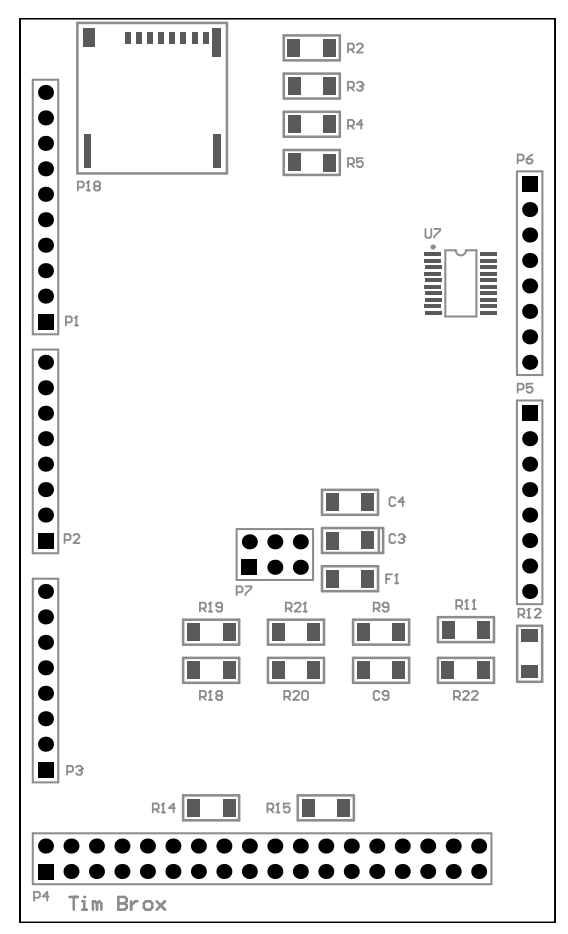

(b) Bottom

Figure C.5: rheoDueShield v4 PCB component layout 


\begin{tabular}{|c|c|c|c|c|}
\hline Description & Designator & Quantity & Digikey PN & Value \\
\hline CAP TANT 10UF 16V 20\% 1206 & $\mathrm{C3}, \mathrm{C5}, \mathrm{C7}$ & & $399-3687-1-N D$ & $10 \mathrm{uF}$ \\
\hline \begin{tabular}{|l|} 
CAP CER 0.1UF 50V 10\% X7R \\
1206
\end{tabular} & $\triangle 4, C 6, C 8, \subset 9$ & & |399-1249-1-ND & $0.1 \mathrm{uF}$ \\
\hline $\begin{array}{l}\text { LED 3.2X1.6MM 590NM YLW } \\
\text { CLR SMD }\end{array}$ & \begin{tabular}{|l}
$\mathrm{D1}$, D2, D3, D4, D5, \\
$\mathrm{D} 7, \mathrm{D} 8$
\end{tabular} & & 754-1144-1-ND & \\
\hline \begin{tabular}{|l|} 
PTC RESET 30V .160A SMD \\
1206
\end{tabular} & $\mathrm{~F} 1, \mathrm{~F} 2$ & & F3132CT-ND & \\
\hline $\begin{array}{l}\text { CONN HDR BRKWAY.100 } \\
\text { 40POS VERT }\end{array}$ & $\begin{array}{l}\text { P1, P2, P3, P4, P5, } \\
\text { P6 }\end{array}$ & & A26509-40-ND & \\
\hline \begin{tabular}{|l|} 
Connector Header 6 Position \\
0.100" $(2.54 \mathrm{~mm})$ Tin Through \\
Hole \\
\end{tabular} & P7 & & S7071-ND & \\
\hline $\begin{array}{l}\text { CONN POWER JACK } \\
2.5 \times 5.5 \mathrm{MM} \mathrm{HI} \mathrm{CUR} \\
\end{array}$ & P8, P9 & & CP-002BHPJCT-ND & \\
\hline $\begin{array}{l}\text { CONN HEADER PH TOP 4POS } \\
\text { 2MM }\end{array}$ & P10 & & $455-1706-N D$ & \\
\hline $\begin{array}{l}\text { CONN HEADER PH TOP 5POS } \\
\text { 2MM }\end{array}$ & P11, P12 & & $455-1707-N D$ & \\
\hline $\begin{array}{l}\text { Connector Header } 12 \text { Position } \\
\text { o.100" (2.54mm) Tin Through } \\
\text { Hole }\end{array}$ & P13 & & S7074-ND & \\
\hline \begin{tabular}{|l|} 
Connector Header 4 Position \\
$0.100 "(2.54 \mathrm{~mm})$ Tin Through \\
Hole
\end{tabular} & P14 & & S7070-ND & \\
\hline $\begin{array}{l}\text { CONN SMB JACK STR } 50 \text { OHM } \\
\text { PCB }\end{array}$ & P15, P16, P17 & & A4041-ND & \\
\hline $\begin{array}{l}\text { CONN MICRO SD CARD PUSH- } \\
\text { PUSH R/A }\end{array}$ & P18 & & WM6377CT-ND & \\
\hline $\begin{array}{l}\text { CONN AUDIO JACK MONO } \\
\text { 3.5MM SMD } \\
\end{array}$ & P19 & & CP-3523MJCT-ND & \\
\hline $\begin{array}{l}\text { MOSFET N-CH 50V 220MA SOT } \\
23\end{array}$ & Q1 & & BSS138CT-ND & \\
\hline \begin{tabular}{|l|}
$\begin{array}{l}\text { RES SMD } 68 \text { OHM 5\% 1/4W } \\
1206\end{array}$ \\
\end{tabular} & $\begin{array}{l}\text { R2, R3, R4, R5, R11, } \\
\text { R14, R15 }\end{array}$ & & P68ECT-ND & 68 \\
\hline $\begin{array}{l}\begin{array}{l}\text { RES SMD } 18 \mathrm{~K} \text { OHM } 5 \% 1 / 4 \mathrm{~W} \\
1206\end{array} \\
\end{array}$ & R6 & & P18KECT-ND & $18 \mathrm{~K}$ \\
\hline 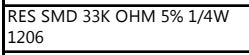 & R7 & & P33KECT-ND & $33 \mathrm{~K}$ \\
\hline $\begin{array}{l}\text { RES SMD 5.1K OHM 5\% 1/4W } \\
1206 \\
\end{array}$ & $\mathrm{R} 9$ & & P5.1KECT-ND & $5.1 \mathrm{~K}$ \\
\hline $\begin{array}{l}\begin{array}{l}\text { RES SMD } 82 \text { OHM 5\% 1/4W } \\
1206\end{array} \\
\end{array}$ & R12 & & P82ECT-ND & 82 \\
\hline 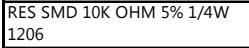 & R16, R17, R22 & & P1OKECT-ND & $10 \mathrm{~K}$ \\
\hline $\begin{array}{l}\text { RES SMD 51K OHM 5\% 1/4W } \\
1206\end{array}$ & R18, R20 & & P51KECT-ND & $51 \mathrm{~K}$ \\
\hline $\begin{array}{l}\text { RES SMD 100K OHM 5\% 1/4W } \\
1206\end{array}$ & R19, R21 & & P100KECT-ND & $100 \mathrm{~K}$ \\
\hline $\begin{array}{l}500 \mathrm{~mA} \text { Low Dropout } \\
\text { Regulator, 4-pin SOT-223, Pb- } \\
\text { Free }\end{array}$ & u1 & & $\begin{array}{l}\text { LM2937IMP- } \\
\text { 12/NOPBCT-ND }\end{array}$ & \\
\hline \begin{tabular}{|l|}
$\begin{array}{l}\text { OPTOISO 7.5KV TRANS } \\
\text { W/BASE 6SMD }\end{array}$ \\
\end{tabular} & uз & & 4N26SM-ND & \\
\hline $\begin{array}{l}500 \text { mA Low Dropout } \\
\text { Regulator, 4-pin SOT-223, Pb- } \\
\text { Free }\end{array}$ & U4 & & $\begin{array}{l}\text { LM2937IMP- } \\
\text { 5.0/NOPBCT-ND }\end{array}$ & \\
\hline \begin{tabular}{|l|} 
General Purpose, Low \\
Voltage, Rail-to-Rail Output \\
Operational Amplifiers, 8-pin \\
Mini SOIC, Pb-Free \\
\end{tabular} & $45, \mathrm{U} 6$ & & 296-9571-1-ND & \\
\hline IC 8-BIT TRNSTR 15KV ESD 20TS & & & 296-21527-1-ND & \\
\hline
\end{tabular}

Figure C.6: rheoDueShield v4 bill of material 


\section{C.2 Torque Variant Electronics}

\begin{tabular}{lccc} 
Part & Schematic & Component Layout & Bill of Material \\
\hline rheoDueShield (Torque Variant) & Figure C.7 & Figure C.8 & Figure C.9 \\
rheoInterface & Figure C.10 & Figure C.11 & Figure C.12 \\
rheoPower & Figure C.13 & Figure C.14 & Figure C.15
\end{tabular}




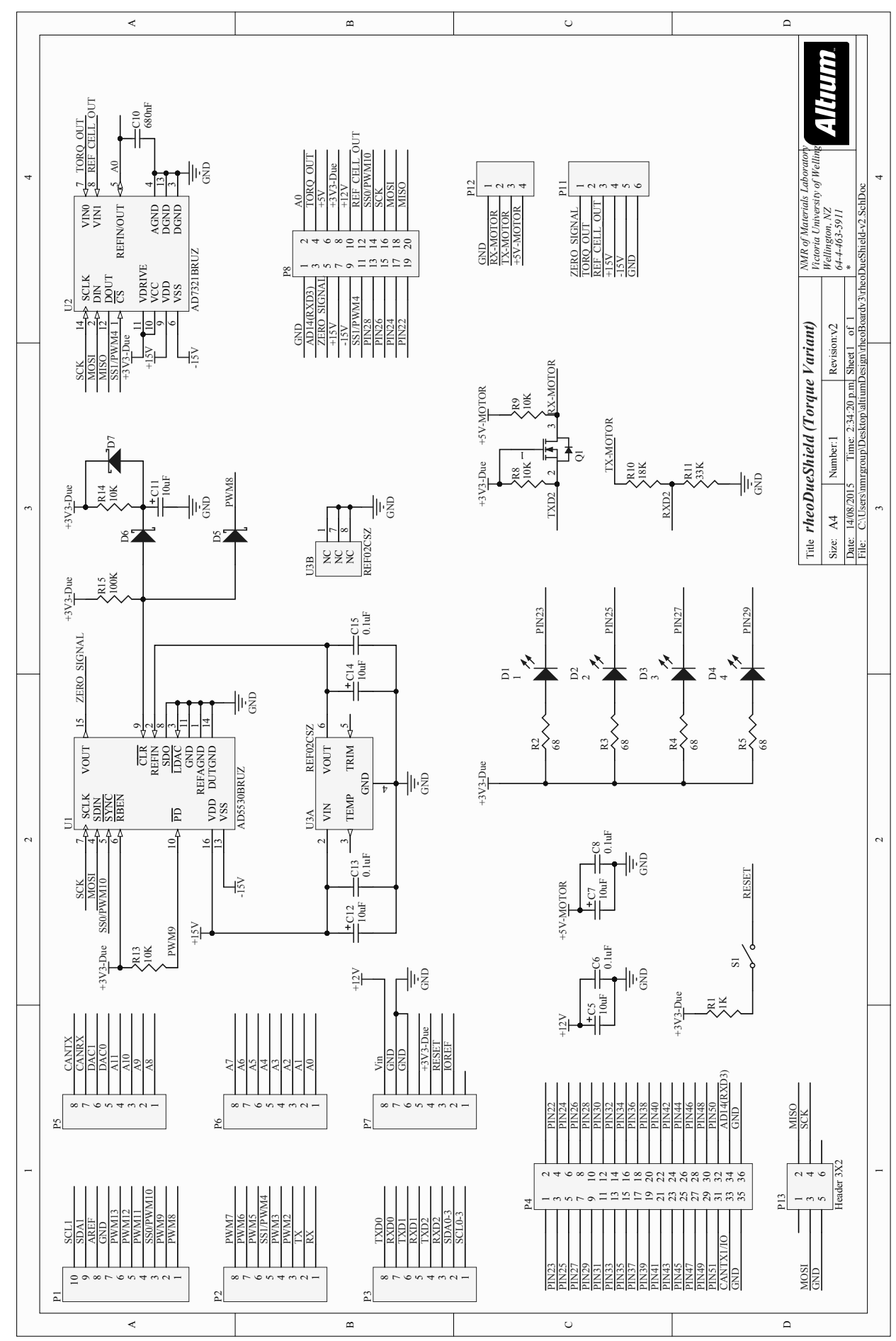

Figure C.7: rheoDueShield v2 (torque variant) schematic 


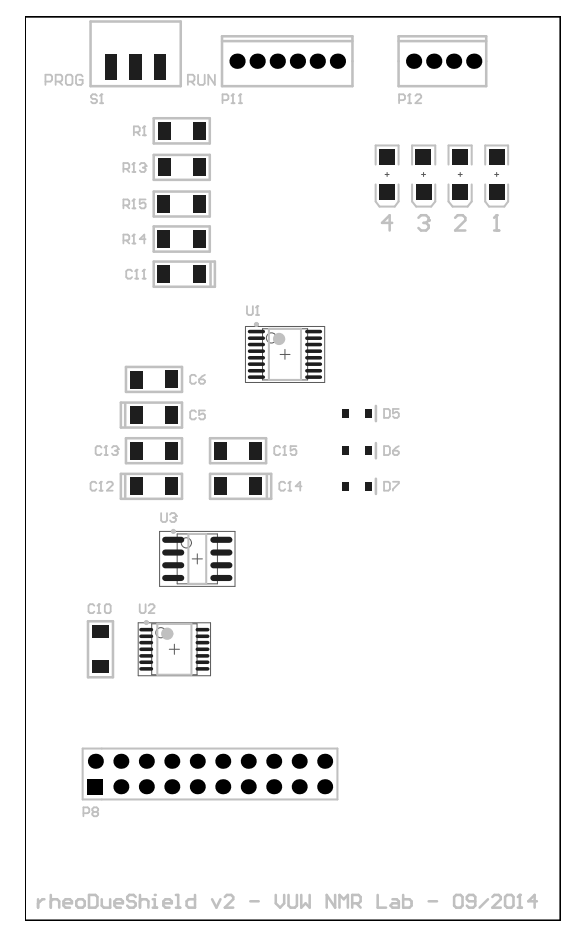

(a) Top

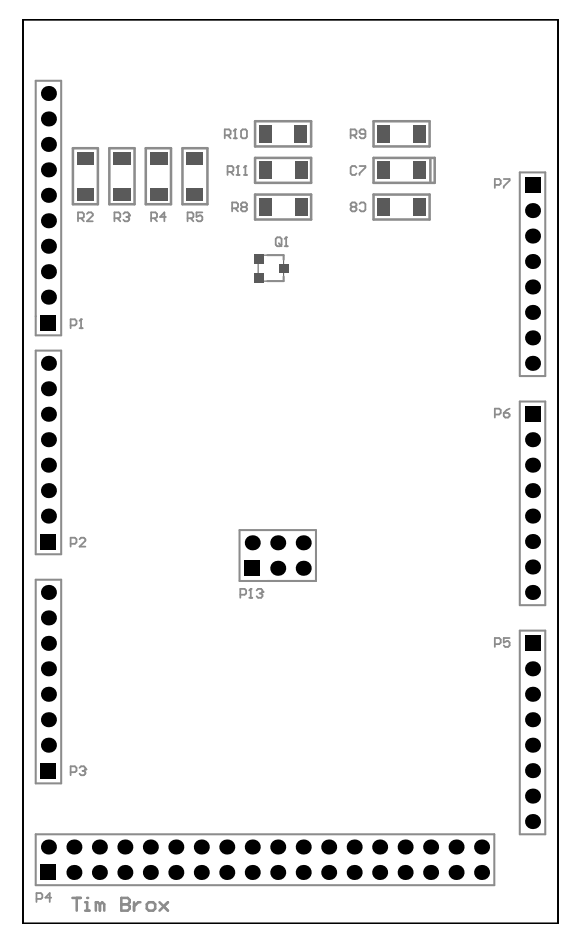

(b) Bottom

Figure C.8: rheoDueShield v2 (torque variant) PCB component layout 


\begin{tabular}{|c|c|c|c|c|}
\hline \begin{tabular}{|c} 
Description \\
\end{tabular} & Designator & Quantity & Digikey PN & Value \\
\hline \begin{tabular}{|l|} 
CAPT TANT 10UF 16V $20 \%$ \\
1206
\end{tabular} & $\mathrm{C} 5, \mathrm{C} 7, \mathrm{C} 11, \mathrm{C} 12, \mathrm{C} 14$ & & 399-3687-1-ND & $10 \mathrm{uF}$ \\
\hline $\begin{array}{l}\text { CAP CER } 0.1 \text { UF } 50 \mathrm{~V} 10 \% \text { X7R } \\
1206\end{array}$ & $\mathrm{C} 6, \mathrm{C} 8, \mathrm{C} 13, \mathrm{C} 15$ & & 4 399-1249-1-ND & $0.1 \mathrm{uF}$ \\
\hline \begin{tabular}{|l|} 
CAP CER 0.68UF 50V 10\% \\
X7R 1206
\end{tabular} & $\mathrm{C} 10$ & & 445-4013-1-ND & $680 \mathrm{nF}$ \\
\hline $\begin{array}{l}\text { LED 3.2X1.6MM 590NM YLW } \\
\text { CLR SMD }\end{array}$ & $\mathrm{D1}, \mathrm{D2}, \mathrm{D} 3, \mathrm{D} 4$ & & 4 754-1144-1-ND & \\
\hline $\begin{array}{l}\text { DIODE SCHOTTKY 7OV 70MA } \\
\text { SOD } 323\end{array}$ & $\mathrm{D} 5, \mathrm{D} 6, \mathrm{D} 7$ & & 497-6652-1-ND & \\
\hline \begin{tabular}{|l|} 
CONN HDR BRKWAY .100 \\
40POS VERT
\end{tabular} & $\mathrm{P1} 1, \mathrm{P2}, \mathrm{P3}, \mathrm{P} 4, \mathrm{P} 5, \mathrm{P} 6, \mathrm{P} 7$ & & A26509-40-ND & \\
\hline \begin{tabular}{|l} 
Connector Receptacle 20 \\
Position 0.100" (2.54mm) \\
Gold Through Hole \\
\end{tabular} & P8 & & SAM1196-10-ND & \\
\hline $\begin{array}{l}\text { CONN HEADER PH TOP } \\
\text { 6POS 2MM }\end{array}$ & P11 & & 1) 455-1708-ND & \\
\hline $\begin{array}{l}\text { CONN HEADER PH TOP } \\
\text { APOS } 2 M M \\
\end{array}$ & P12 & & 455-1706-ND & \\
\hline \begin{tabular}{|l|} 
Connector Header 6 \\
Position 0.1.00" (2.54mm) Tin \\
Through Hole
\end{tabular} & P13 & & S7071-ND & \\
\hline $\begin{array}{l}\begin{array}{l}\text { MOSFET N-CH 50V 220MA } \\
\text { SOT-23 }\end{array} \\
\end{array}$ & Q1 & & BSS138CT-ND & \\
\hline $\begin{array}{l}\begin{array}{l}\text { RES SMD 1K OHM 5\% 1/4W } \\
1206\end{array} \\
\end{array}$ & R1 & & P1.OKECT-ND & $1 \mathrm{~K}$ \\
\hline \begin{tabular}{|l|l|}
$\begin{array}{l}\text { EES SMD } 68 \text { OHM 5\% 1/4W } \\
1206\end{array}$ \\
\end{tabular} & R2, R3, R4, R5 & & 4 P68ECT-ND & 68 \\
\hline \begin{tabular}{|l|l|l}
$\begin{array}{l}\text { RES SMD 10K OHM 5\% 1/4W } \\
1206\end{array}$ \\
\end{tabular} & R8, R9, R13, R14 & & 4 P1OKECT-ND & $10 \mathrm{~K}$ \\
\hline $\begin{array}{l}\begin{array}{l}\text { RES SMD 18K OHM 5\% 1/4W } \\
1206\end{array} \\
\end{array}$ & R10 & & 1 P18KECT-ND & $18 \mathrm{~K}$ \\
\hline $\begin{array}{l}\begin{array}{l}\text { RES SMD 33K OHM 5\% 1/4W } \\
1206\end{array} \\
\end{array}$ & R11 & & 1 P33КЕCT-ND & $33 \mathrm{~K}$ \\
\hline \begin{tabular}{l|}
$\begin{array}{l}\text { RES SMD } 100 K \text { OHM 5\% } \\
\text { 1/4W } 1206\end{array}$ \\
\end{tabular} & R15 & & P100KECT-ND & $100 \mathrm{~K}$ \\
\hline $\begin{array}{l}\text { SWITCH SLDE SPDT 300MA } \\
6 \mathrm{~V}\end{array}$ & s1 & & 401-1999-1-ND & \\
\hline $\begin{array}{l}\begin{array}{l}\text { IC DAC 12BIT SRL IN/NOUT } \\
\text { 16TSSOP }\end{array} \\
\end{array}$ & u1 & & 1 AD5530BRUZ-ND & \\
\hline $\begin{array}{l}\text { IC ADC 12BIT+ SAR 2CHAN } \\
\text { 14TSSOP }\end{array}$ & u2 & & AD7321BRUZ-ND & \\
\hline IC VREF SERIES 5 V 8SOIC & & & & \\
\hline
\end{tabular}

Figure C.9: rheoDueShield v2 (torque variant) bill of material 


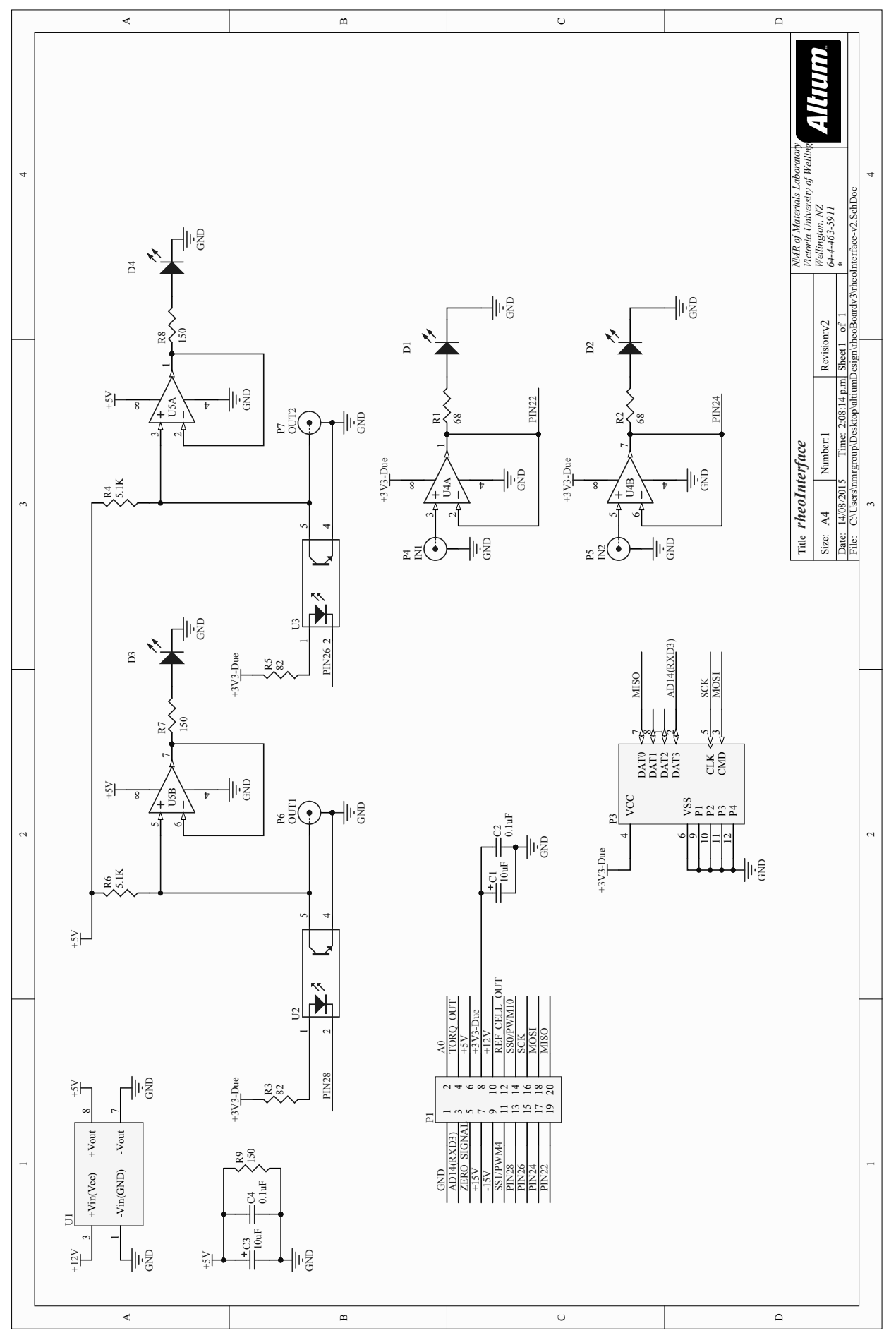

Figure C.10: rheoInterface v2 (torque variant) schematic 


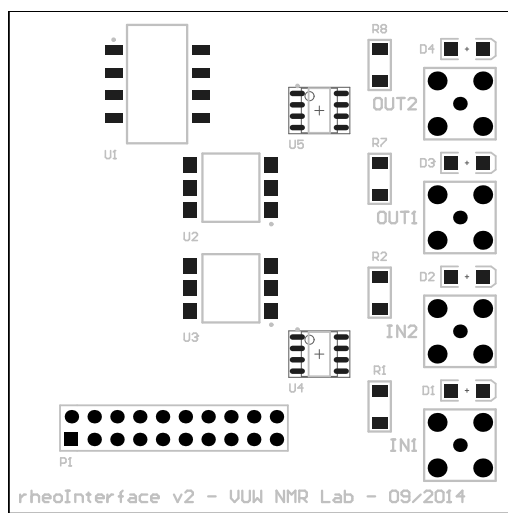

(a) Top

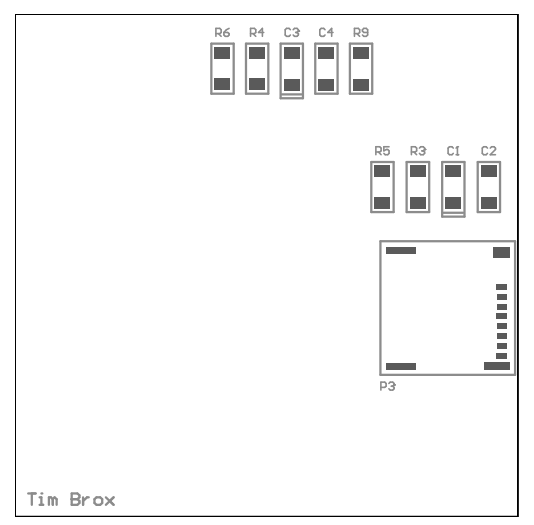

(b) Bottom

Figure C.11: rheoInterface v2 (torque variant) PCB component layout

\begin{tabular}{|c|c|c|c|c|}
\hline Description & Designator & Quantity & Digikey PN & Value \\
\hline $\begin{array}{l}\text { CAP TANT 10UF 16V 20\% } \\
1206\end{array}$ & $\mathrm{C} 1, \mathrm{C3}$ & & 399-3687-1-ND & 10uF \\
\hline $\begin{array}{l}\text { CAP CER 0.1UF 50V 10\% X7R } \\
1206\end{array}$ & $\mathrm{C} 2, \mathrm{C} 4$ & & 399-1249-1-ND & $0.1 \mathrm{uF}$ \\
\hline $\begin{array}{l}\text { LED 3.2X1.6MM 590NM YLW } \\
\text { CLR SMD }\end{array}$ & D1, D2, D3, D4 & & 754-1144-1-ND & \\
\hline $\begin{array}{l}\text { Connector Receptacle } 20 \\
\text { Position } 0.100 "(2.54 \mathrm{~mm}) \\
\text { Gold Through Hole }\end{array}$ & P1 & & SAM1196-10-ND & \\
\hline $\begin{array}{l}\text { CONN MICRO SD CARD } \\
\text { PUSH-PUSH R/A }\end{array}$ & P3 & & WM6377CT-ND & \\
\hline $\begin{array}{l}\text { CONN SMB JACK R/A } 50 \\
\text { OHM PCB }\end{array}$ & P4, P5, P6, P7 & & A101970-ND & \\
\hline $\begin{array}{l}\text { RES SMD } 68 \text { OHM 5\% 1/4W } \\
1206\end{array}$ & R1, R2 & & P68ECT-ND & 68 \\
\hline $\begin{array}{l}\text { RES SMD } 82 \text { OHM 5\% 1/4W } \\
1206\end{array}$ & R3, R5 & & P82ECT-ND & 82 \\
\hline $\begin{array}{l}\text { RES SMD 5.1K OHM 5\% } \\
\text { 1/4W } 1206\end{array}$ & R4, R6 & & P5.1KECT-ND & $5.1 \mathrm{~K}$ \\
\hline $\begin{array}{l}\text { RES SMD } 150 \text { OHM 5\% 1/4W } \\
1206\end{array}$ & $R 7, R 8, R 9$ & & P150ECT-ND & 150 \\
\hline $\begin{array}{l}\text { CONV DC/DC 1W 12VIN 5V } \\
\text { SGL } 1 \mathrm{KV}\end{array}$ & U1 & & 811-1685-5-ND & \\
\hline $\begin{array}{l}\text { OPTOISO 7.5KV TRANS } \\
\text { W/BASE 6SMD }\end{array}$ & U2, U3 & & $4 \mathrm{~N} 26 \mathrm{SM}-\mathrm{ND}$ & \\
\hline IC OPAMP GP 1MHZ RRO 8SC & U4, U5 & & 296-9571-1-ND & \\
\hline
\end{tabular}

Figure C.12: rheoInterface v2 (torque variant) bill of material 


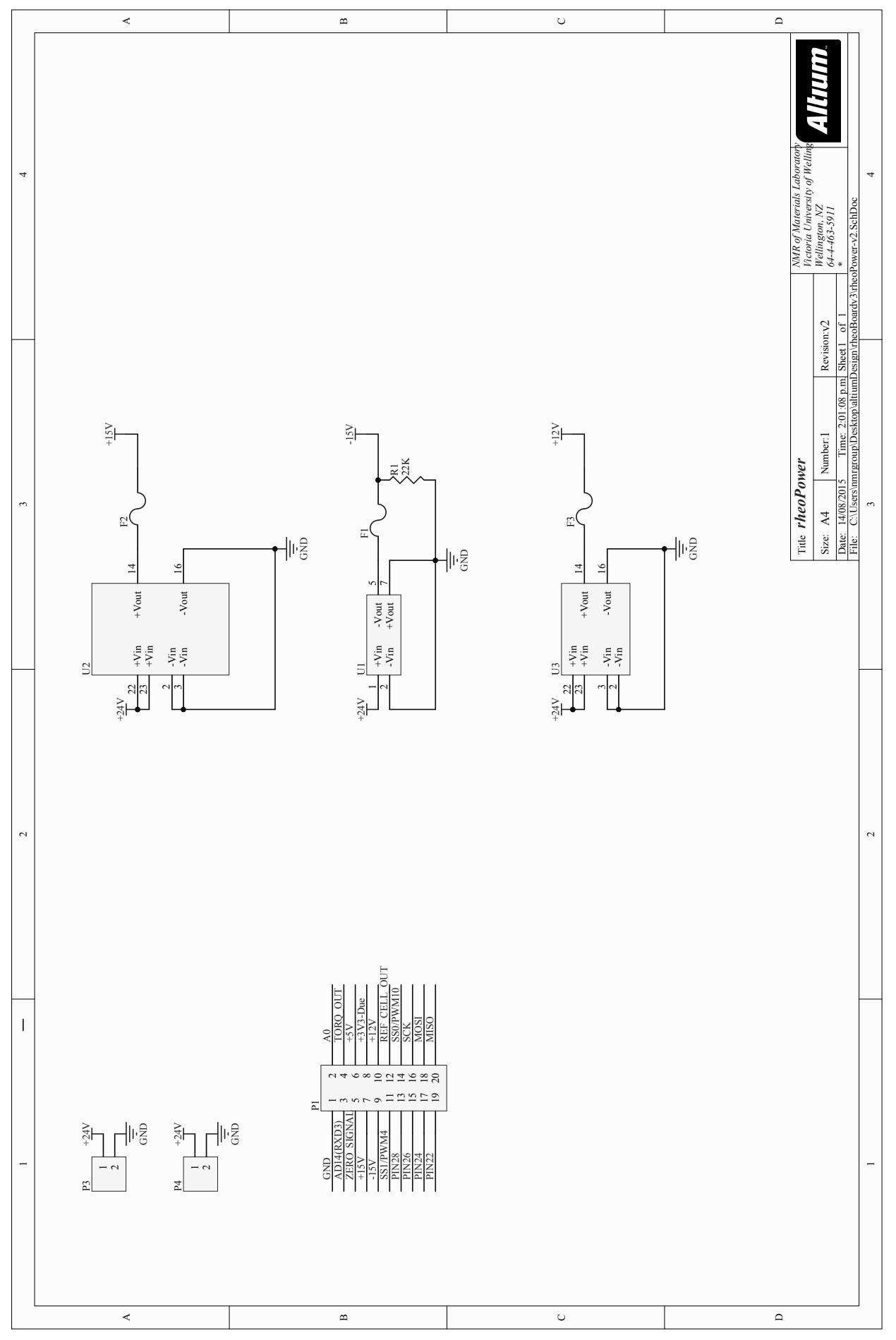

Figure C.13: rheoPower v2 (torque variant) schematic 


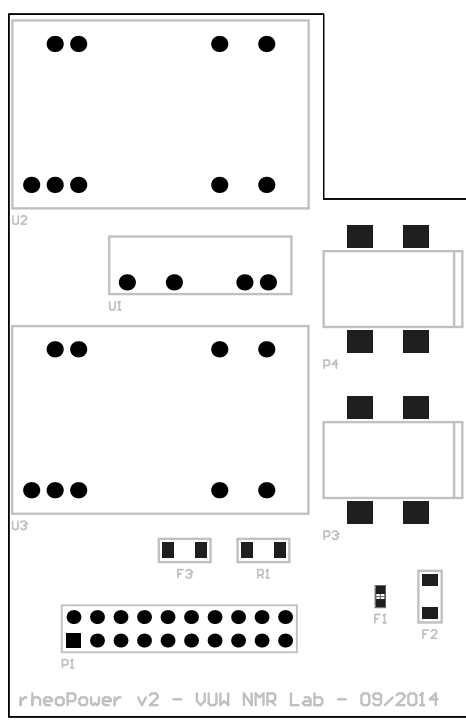

(a) Top

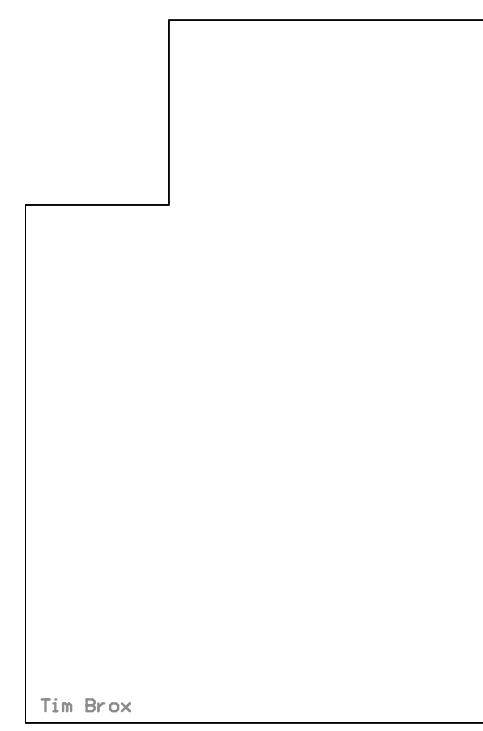

(b) Bottom

Figure C.14: rheoPower v2 (torque variant) PCB component layout

\begin{tabular}{|c|c|c|c|c|}
\hline Description & Designator & Quantity & Digikey PN & Value \\
\hline $\begin{array}{l}\text { THERMISTOR PTC } 47 \text { OHM } \\
\text { SMD }\end{array}$ & F1 & & $\begin{array}{ll}1 & 490-2472-1-N D\end{array}$ & \\
\hline $\begin{array}{l}\text { FUSE PTC RESET 16V .25A } \\
1206\end{array}$ & F2 & & 1 F $2110 C T-N D$ & \\
\hline $\begin{array}{l}\text { FUSE PTC RESET 24V .20A } \\
1206\end{array}$ & F3 & & 1 F2109CT-ND & \\
\hline \begin{tabular}{|l|} 
Connector Receptacle 20 \\
Position 0.100" (2.54mm) \\
Gold Through Hole
\end{tabular} & P1 1 & & 1 SAM1196-10-ND & \\
\hline $\begin{array}{l}\text { CONN POWER JACK } \\
\text { 2.5X5.5MM HI CUR }\end{array}$ & P3, P4 & & 2 CP-002BHPJCT-ND & \\
\hline $\begin{array}{l}\text { RES SMD 22K OHM 5\% 1/4W } \\
1206\end{array}$ & R1 & & 1 P22KECT-ND & $22 \mathrm{~K}$ \\
\hline DC/DC CONVERTER 15V $1 \mathrm{~W}$ & U1 & & \begin{tabular}{l|l|}
1 & $811-2162-5-N D$ \\
\end{tabular} & \\
\hline $\begin{array}{l}\text { CONV DC/DC 8W 24VIN } \\
\text { 15VOUT }\end{array}$ & U2 & & 1 945-1907-5-ND & \\
\hline CONV DC/DC 5W 18-36VIN 1 & U3 & & 1 945-1481-5-ND & \\
\hline
\end{tabular}

Figure C.15: rheoPower v2 (torque variant) bill of material 


\section{Appendix D}

\section{motionControl Serial Commands}

This appendix explains the principles of the interactions between the user (e.g. via motionControl program) and the drive system firmware. These concepts are the core functions necessary for reproducing this interaction whether it be in the Bruker TopSpin environment or a simple Terminal window.

\section{D.1 Motion Commands}

All commands are sent as ASCII strings. The following section can be used to create the various motion command strings recognised by the motionControl system. In the following descriptions, the square brackets and periods would not be included in the command string. These characters are used to help understand the individual components of the motion commands. For illustration purposes an example command has been included under the "Single Value Shear Rate" section.

\section{D.1.1 Stop}

[0]

Stop Motor and Clear All Parameters

The "Stop" motion command will bring the motor to a halt and subsequently clear all motion parameters on the motor electronics. To resume motion after a "Stop" command, a new motion profile command will need to be sent. 


\section{D.1.2 Single Value Shear Rate}

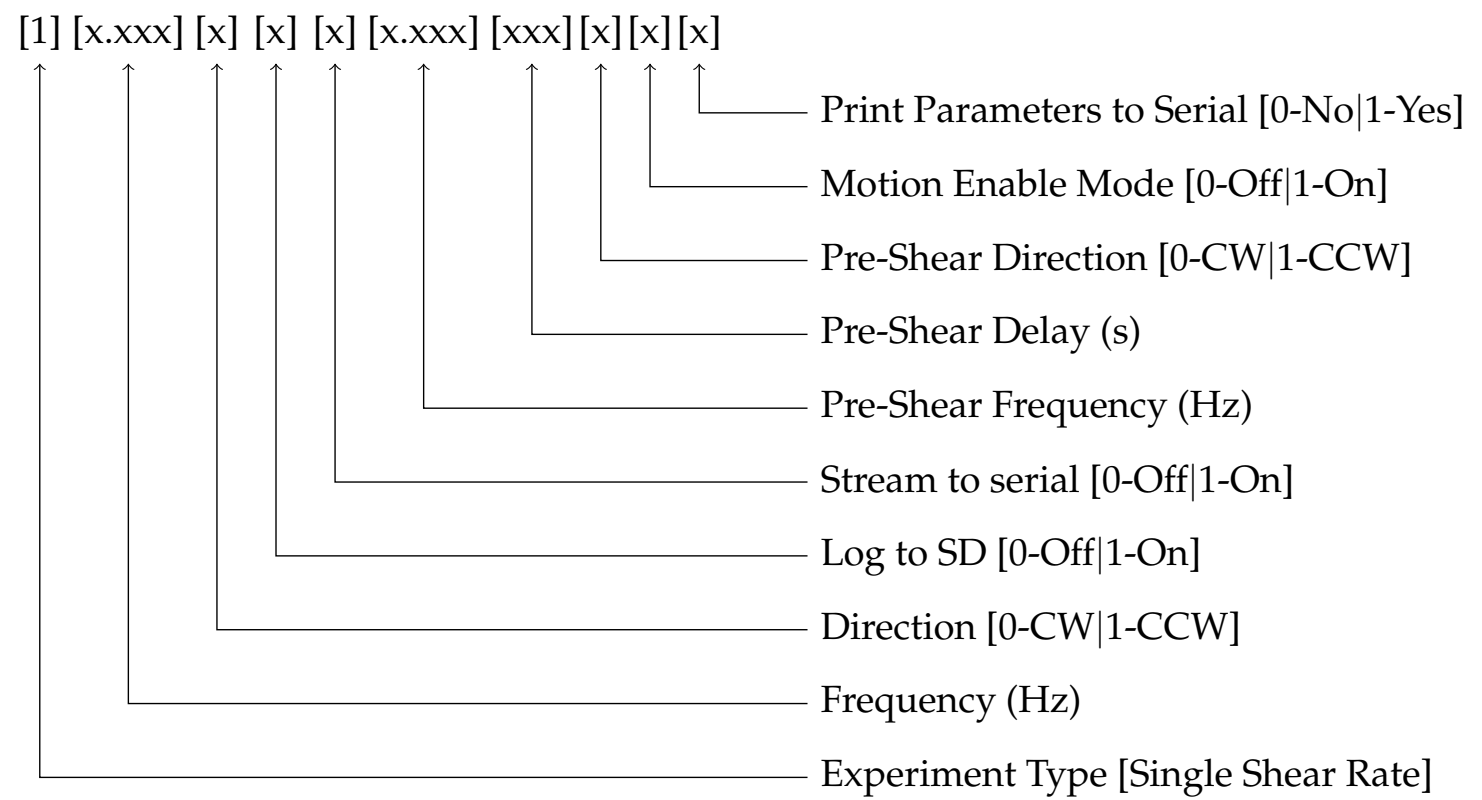

A "Single Value Shear Rate" experiment is a shear rate controlled experiment were the motor is rotated at a fixed frequency. Sending the motionControl electronics a "Single Value Shear Rate" command when the motor is currently moving will cause the motor to step the frequency (either increase or decrease) of rotation without stopping the motor. In this way, the shear rate can be progressively ramped as a function of time via an external programming (e.g. TopSpin automation programme).

\section{Example: "120000106000030001"}

This is a motion command for a "Single Value Shear Rate" experiment at $2.000 \mathrm{~Hz}$. The motor will rotate clockwise and will log to the mounted SD card; data will not be streamed via the serial/USB connection. Prior to the experiment, the sample will be pre-sheared CW at $6.000 \mathrm{~Hz}$ for a period of 30 seconds. The motionEnable mode will be turned off and the motor package will echo the parameters via serial. 


\section{D.1.3 Oscillatory Shear}

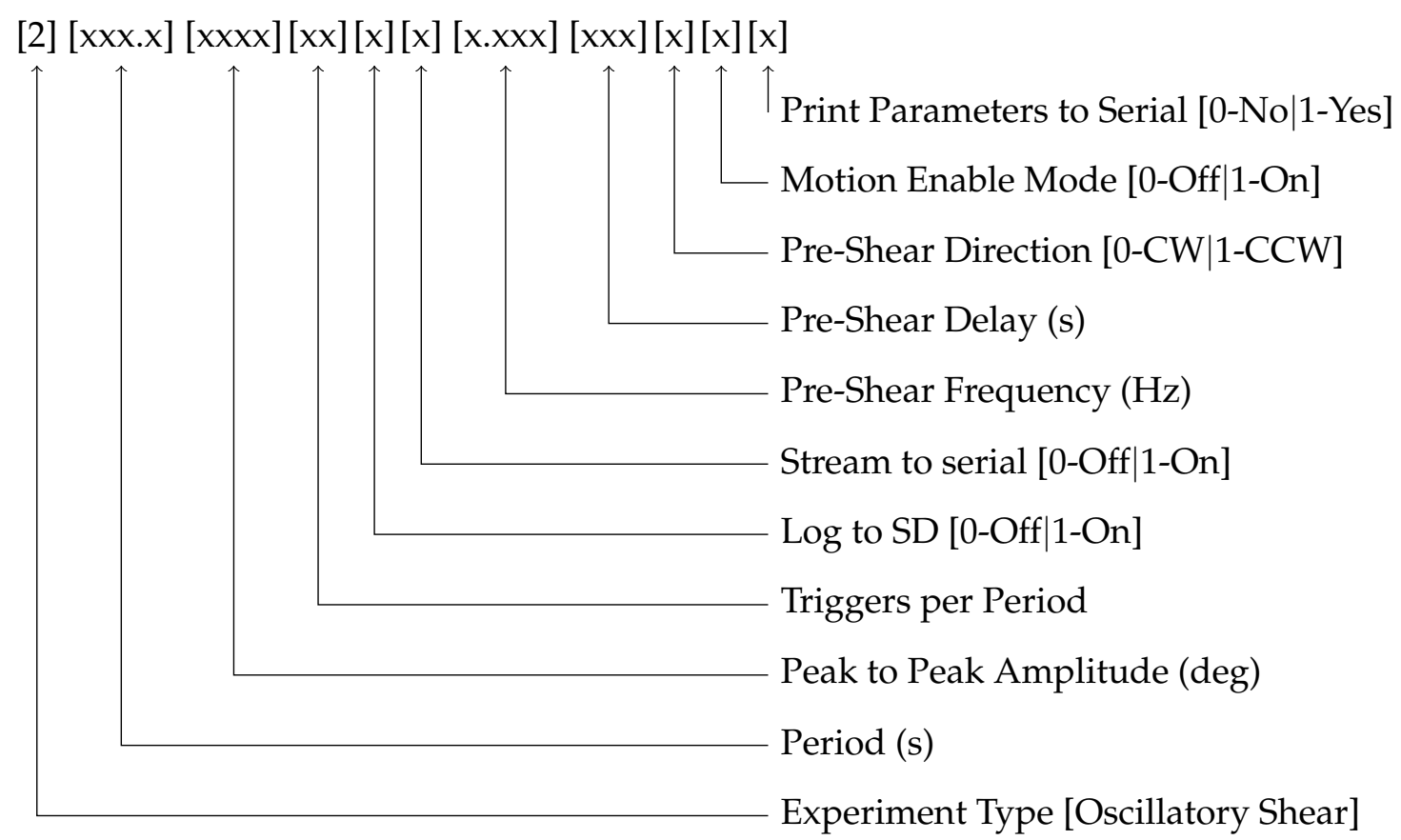

With the "Oscillatory Shear" motion command, the motionControl motor will output an oscillating shear rate profile. Due to motor inertia, the "Oscillatory Shear" profiles will always start from a zero velocity position of the waveform.

\section{D.1.4 Stepped Shear Rate}

$[3][x \cdot x x x][. x x x][x x][x x x][x][x]$

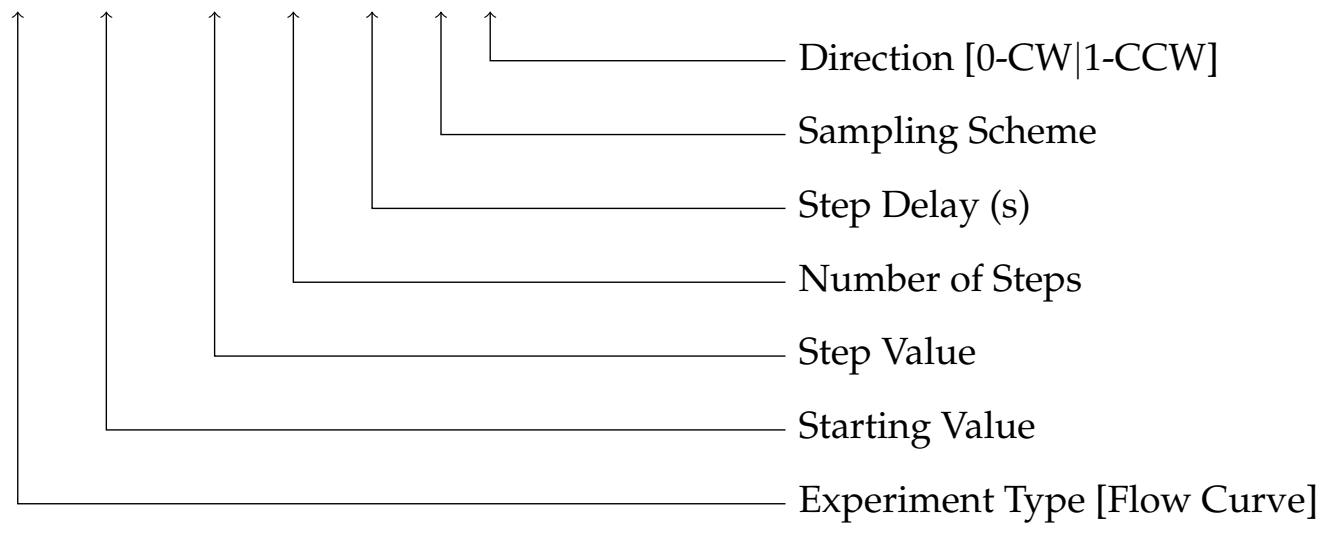




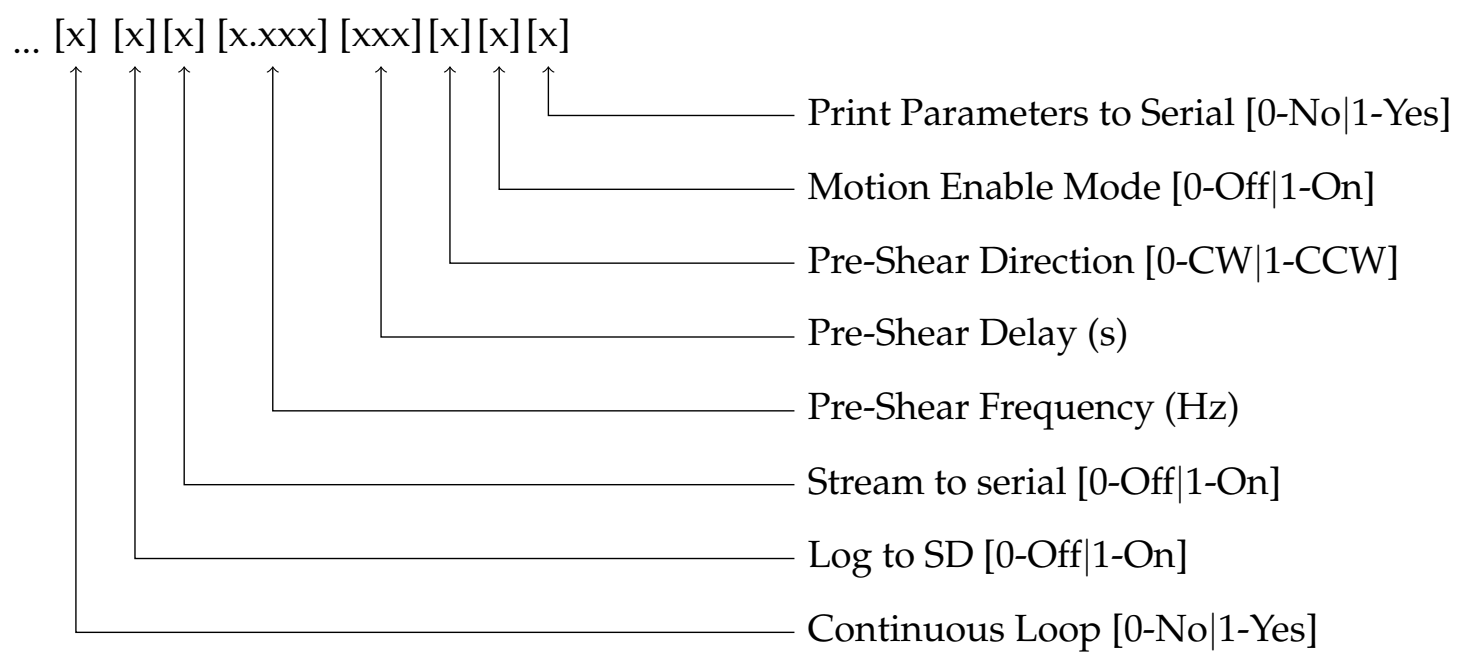

The "Stepped Shear Rate" motion profile is a stepped flow experiment where successive shear rates are applied for a predetermined amount of time. The shear rates sampled during this motion profile can be equally spaced in linear or logarithmic space. As currently programmed, the "Stepped Shear Rate" command can only be used for increasing shear rates. Until the firmware is updated, reverse flow curves (successively decreasing shear rates) can be accomplished by using multiple "Single Value Shear Rate" commands.

\section{D.1.5 Sawtooth}

\section{$[4][x x x][x x . x x x][x x][x][x][x][x]$}

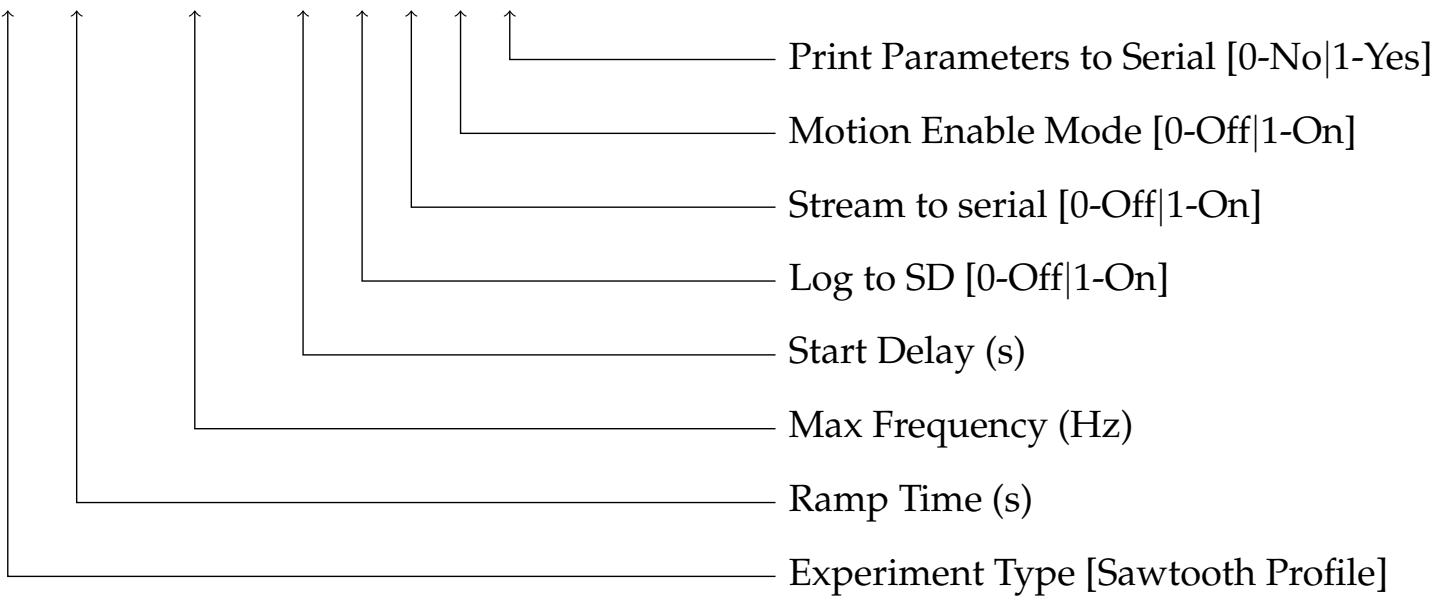

The "Sawtooth" motion profile is used to set the motor rotating at a constant acceleration. Based upon the desired maximum frequency and ramp time the motor calculates an acceleration set point. Once the max frequency has been reached the profile will start again. The start delay parameter can be used to set a delay between successive profiles. 


\section{D.1.6 Square Wave}

$[5][x x . x x x][x x . x x x][x x x][x x x][x][x][x][x][x]$

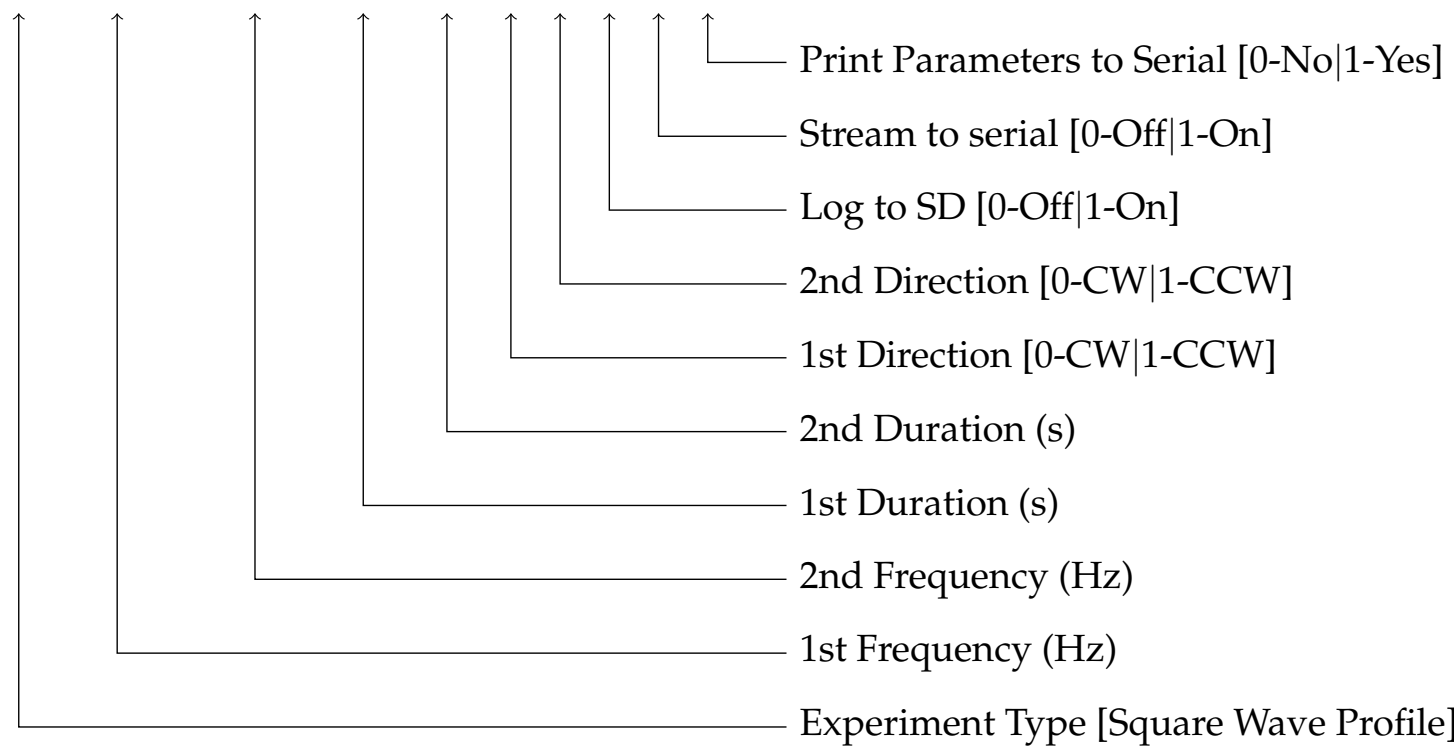

Using the "Square Wave" motion profile, the motor alternates between two frequencies with a variable duty cycle. The direction of rotation can be different for the two frequencies.

\section{D.1.7 Stepped Motion}

$[6][\mathrm{xxxx}][\mathrm{x}]$

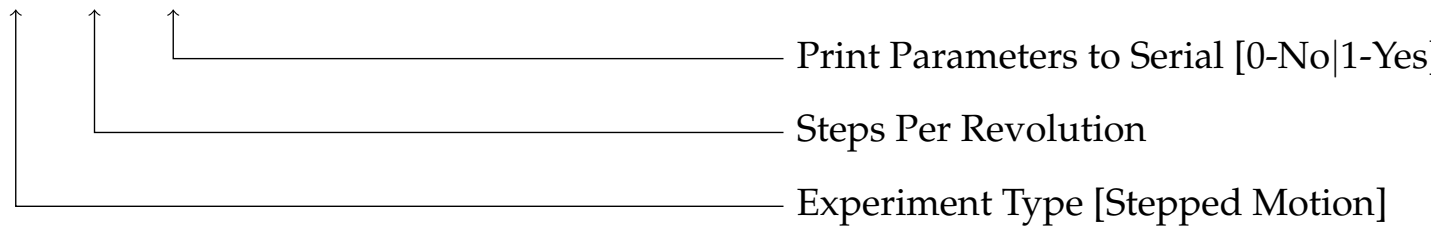

This protocol does not produce continuous motion, but instead increments the motor position by a defined value based upon an external trigger. The "Stepped Motion" routine can be helpful for creating stop-motion movies of a shear cell during one complete rotation to illustrate the amount of mechanical wobble present in the device. 


\section{D.1.8 Single Value Shear Stress}

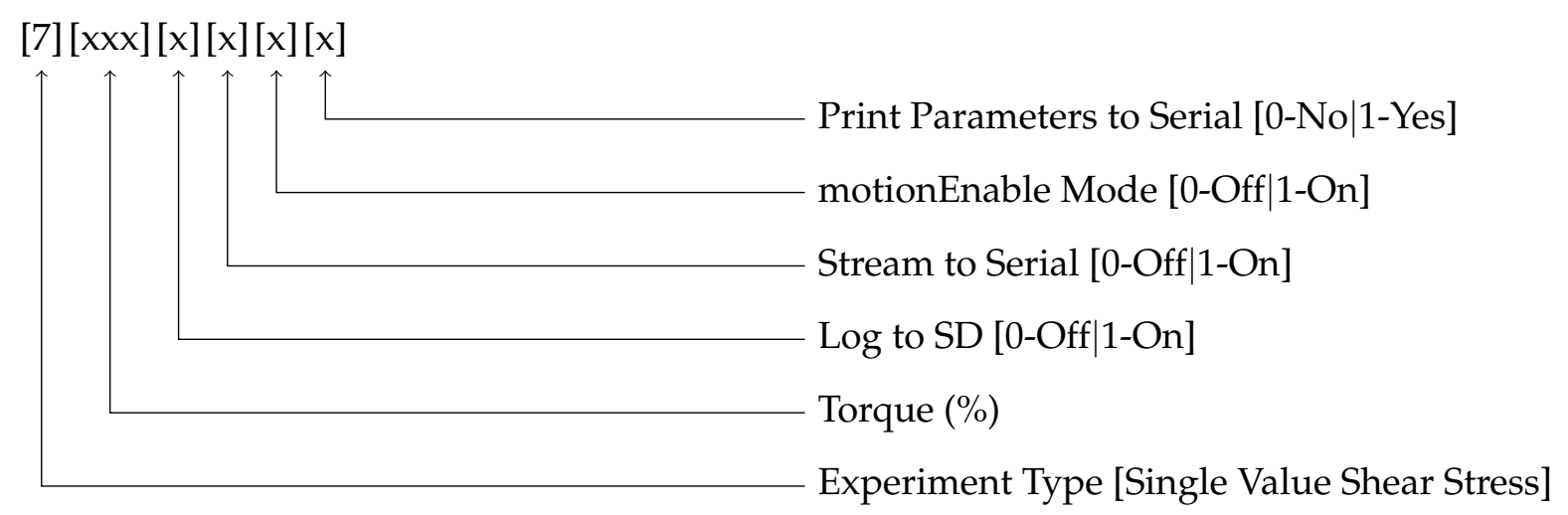

The "Single Value Shear Stress" motion profile applies a fixed torque to the shear cell device. Under this protocol the frequency of the motor is not set. If no sample is loaded (or if the torque is set too high) the motor will continue to accelerate until it reaches its maximum frequency at which it will turn off.

\section{D.2 Parameters and Data Handling Commands}

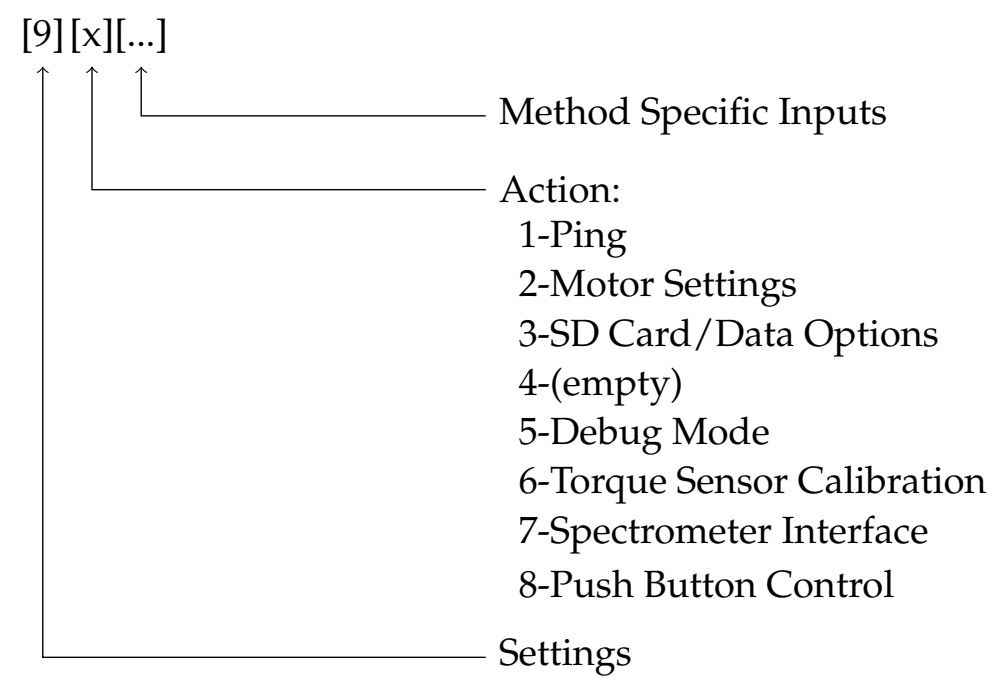




\section{D.2.1 Ping Motor}

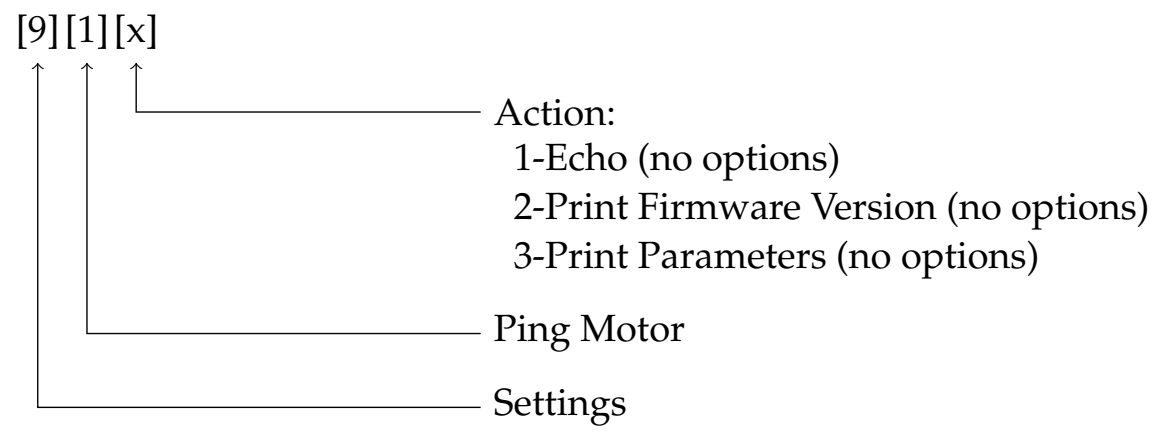

Echo

A command of "911" will cause the motor to send a response. The echo response is not designed to be human readable and is implemented for functions within the graphical user interface programme. If a human readable response is needed a better option would the the "Print Firmware Version" command (see below).

\section{Print Firmware Version}

A command of "912" will cause the motor to print the current firmware version to serial. This function is useful for determining what functions are available on the currently loaded firmware. This can also be useful for bug tracking.

\section{Print Parameters}

A command of "913" will cause the motor to print the current parameters. The first line of the motor response will include the contents of the parameters array (each entry seperated by a comma), the second line be the contents of the preshearParams array and the third line be the contents of the dataParams array. Interpretation of these values is left to the user. 


\section{D.2.2 Motor Settings}

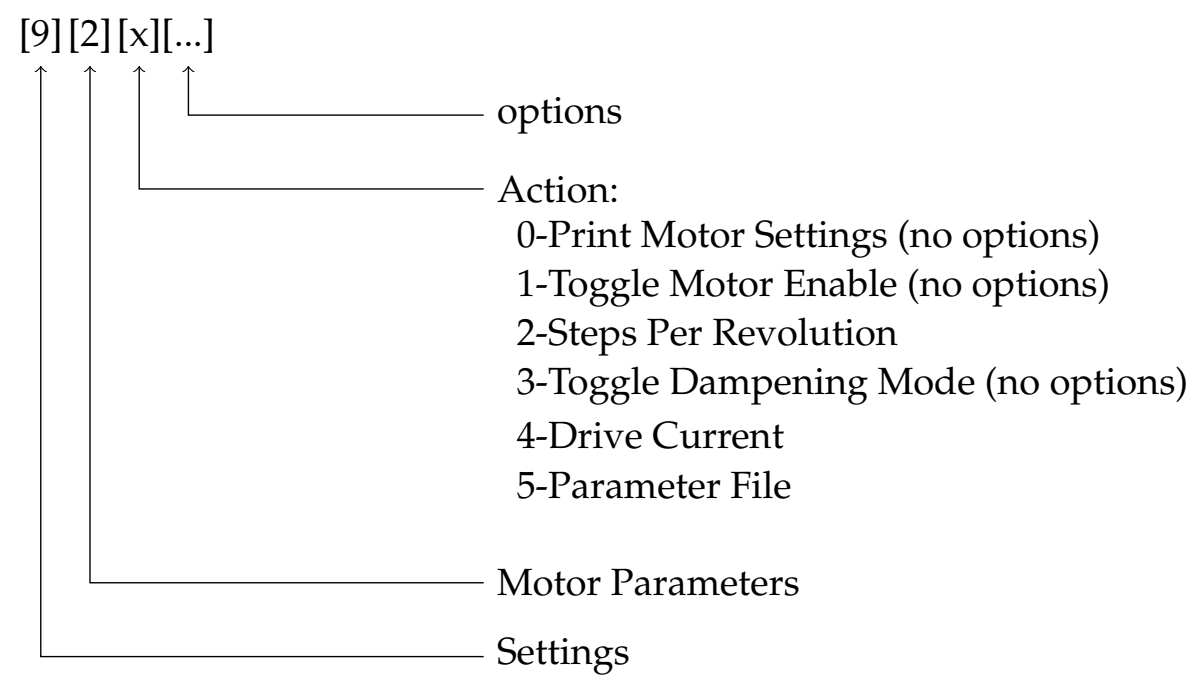

\section{Print Motor Settings}

A command of "920" will print the current motor settings.

\section{Toggle Motor Enable}

A command of "921" will toggle the motor between enable and disable. When the motor is disabled, the motor will not rotate, but will otherwise behave normally. The motor must be enabled for typical usage. This function can be used to test motor interactions when it is essential that the motor can not move (e.g. sensitive sample in shear device).

\section{Steps Per Revolution}

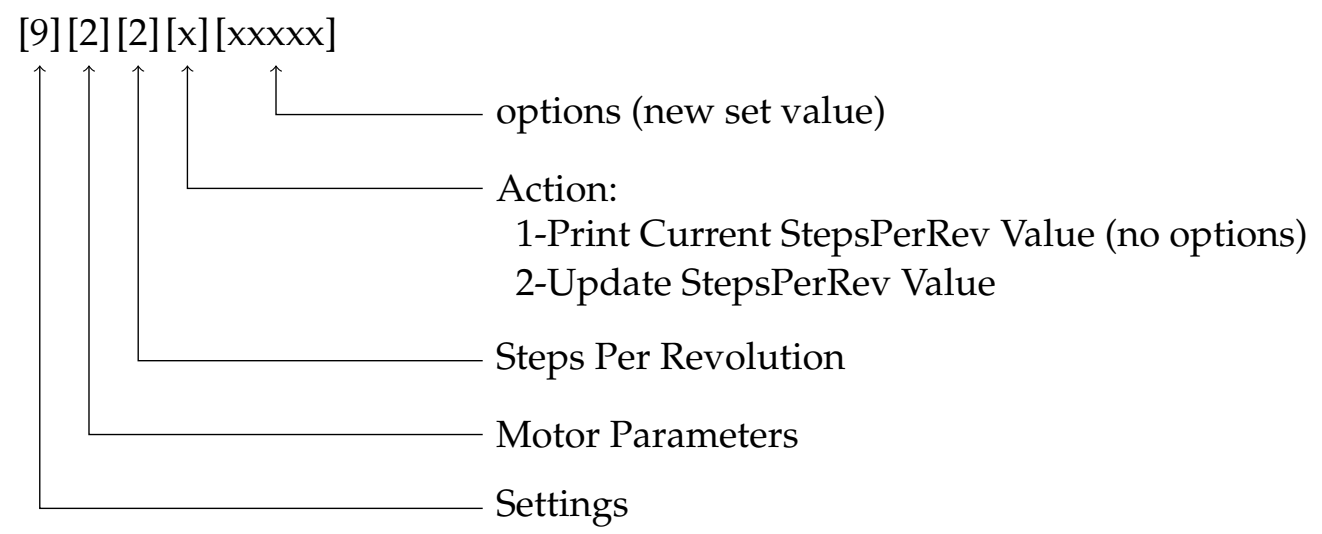

The steps-per-revolution parameter is used to define the step size for the motor. The EvoDrive system will accept a step-per-revolution value from 1 to 102400, however, the motionControl software limits this parameter to 99999. Practically, the finest 
step resolution for the motor is half of the optical encoder resolution. Currently, the motor utilises a 14 bit optical encoder, suggesting the maximum value for the step-perrevolution parameter at 13 bit (8192). Using a steps-per-revolution value greater than half the resolution of the optical encoder will not damage the system, but the performance should not improve.

\section{- Print Current StepsPerRev Value}

A command of "9221" cause the motor to print the current StepsPerRev value.

\section{- Update StepsPerRev Value}

This command is used to update the StepsPerRev parameter. To change the stepsper-revolution parameter used by the motor, use a command of "9222xxxxx" where the five trailing $x^{\prime} s^{\prime}$ are replaced by the desired value. If updating to value with less than five digits (e.g. 8192) add leading zeroes to pad the value (e.g. 08192).

\section{Toggle Damping Mode}

A command of "923" will toggle the dampening mode between enable and disable. From the EvoDuino Programmers Manual: "Low speed damping is active at velocities less than 2 revs per second. Damping mode applies secondary currents to the motor to control the effects of detent torque on motor position." In practice having the damping mode enabled should help produce smoother motion at low frequencies.

\section{Drive Current}

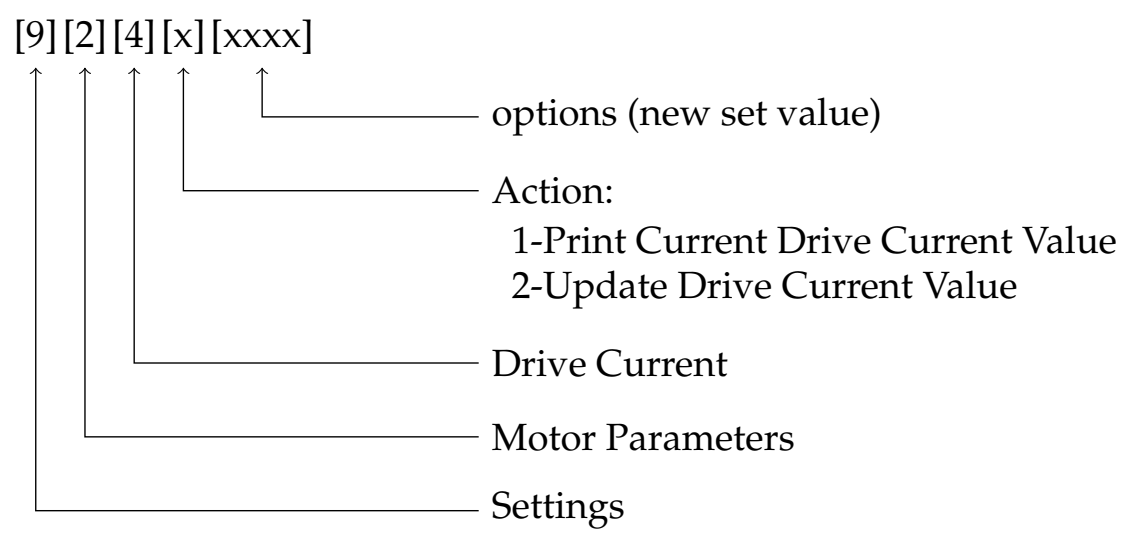

The drive current parameter is used to set the maximum current available to the motor. The stepper motor currently used in the motionControl system has a max current rating of $2800 \mathrm{~mA}$. The absolute maximum current allowed by the EvoDrive electronics is $3000 \mathrm{~mA}$. Changing the maximum drive current is typically used pseudo torque 
control (either to limit the maximum torque output from the motor for safety purposes or to control/measure torque as a rhelogical parameter).

\section{- Print Current Drive Current Value}

A command of "9241" cause the motor to print the current drive current value.

\section{- Update Drive Current Value}

This command is used to update the drive current parameter. To change the drive current parameter (taken as the maximum current available for the motor), us a command of "9242xxxx" where the four trailing x's are replaced by the desire current in milli-amperes (mA). If updating to a value with less than four digits (e.g. 500) add leading zeros to pad the value (e.g. 0500). The absolute maximum current allowed by the EvoDrive electronics is $3000 \mathrm{~mA}$.

\section{Store Parameters}

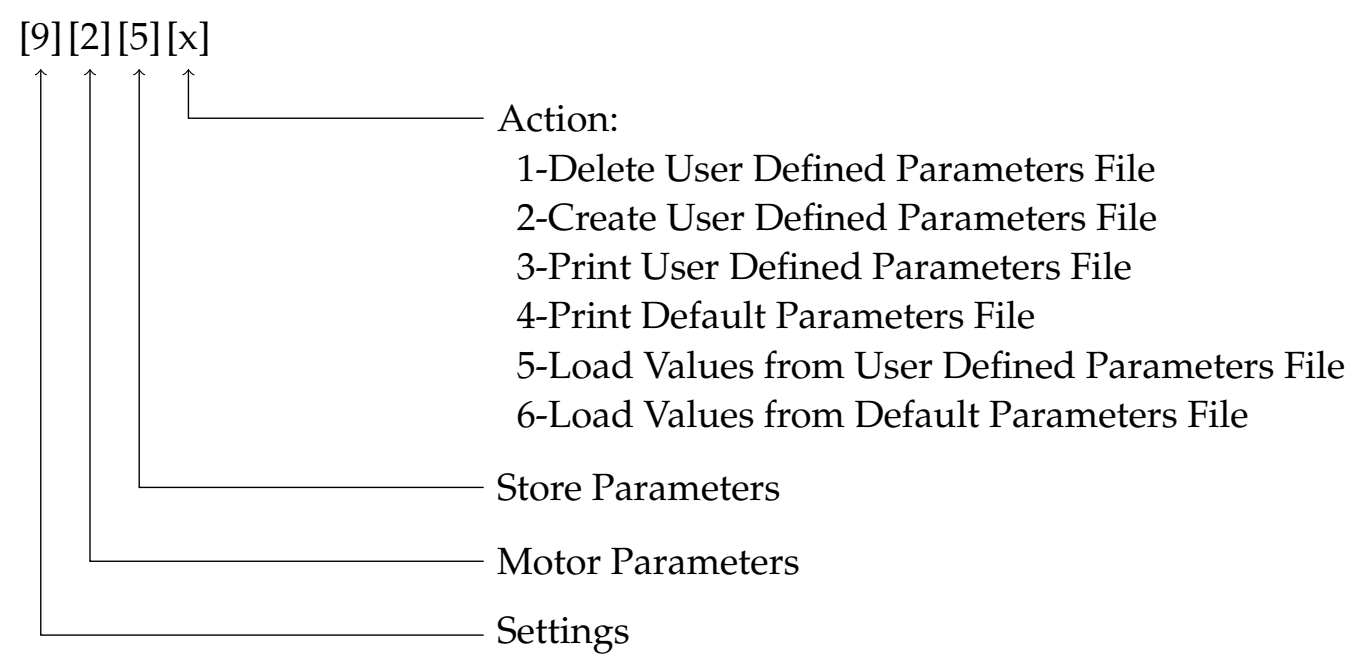

The parameters used in the firmware programme are stored within volatile memory space of the micro-processor. For this reason when the CPU looses power (or is rebooted), changes to these parameters are lost. To make the system easier to use, these values are now saved to the micro SD card mounted on the rheoDueShield. On the SD card there is a directory labelled "settings" which contains two files. The first, "params.txt", is updated whenever a user changes a tacked parameter. Whenever the processor is started, the values in this file are read and are used by the firmware programme. The second file, "default.txt", contains default values which are the initial values programmed into the firmware. The default file can be used to restore a motionControl system to its original settings. If a new SD card is used, the "settings" directory and the two parameter files are created. Initially both will contain the same values until the user changes a parameter. As which parameters are stored in these files may change 
as the firmware evolves, the best method for determining which values are tracked is to print the contents of one of these files (either "9253" or "9254", see below for more information).

\section{- Delete User Defined Parameters File}

A command of "9251" will delete the "settings/params.txt" file. Deleting this file is not a typical operation, but may be necessary if the file has been corrupted.

\section{- Create User Defined Parameters File}

A command of "9252" will create a "settings/params.txt" file on the loaded micro SD card or overwrite the existing file. Whenever a tracked parameter is changed via serial commands, the user defined parameters file is updated, thus the end user does not need to execute this command each time a parameter is changed. Deleting this file is not a typical operation.

\section{- Print User Defined Parameters File}

A command of "9253" will result in the motor printing the contents of the "settings/params.txt" file via serial.

\section{- Print Default Parameters File}

A command of "9254" will result in the motor printing the contents of the "settings/default.txt" file via serial.

\section{- Load Values from User Defined Parameters File}

Upon executing a command of "9255" the motor will update parameters based upon the contents of the "settings/params.txt" file.

\section{- Load Values from Default Parameters File}

Upon executing a command of "9255" the motor will update parameters based upon the contents of the "settings/default.txt" file. 


\section{D.2.3 SD Card/Data Options}

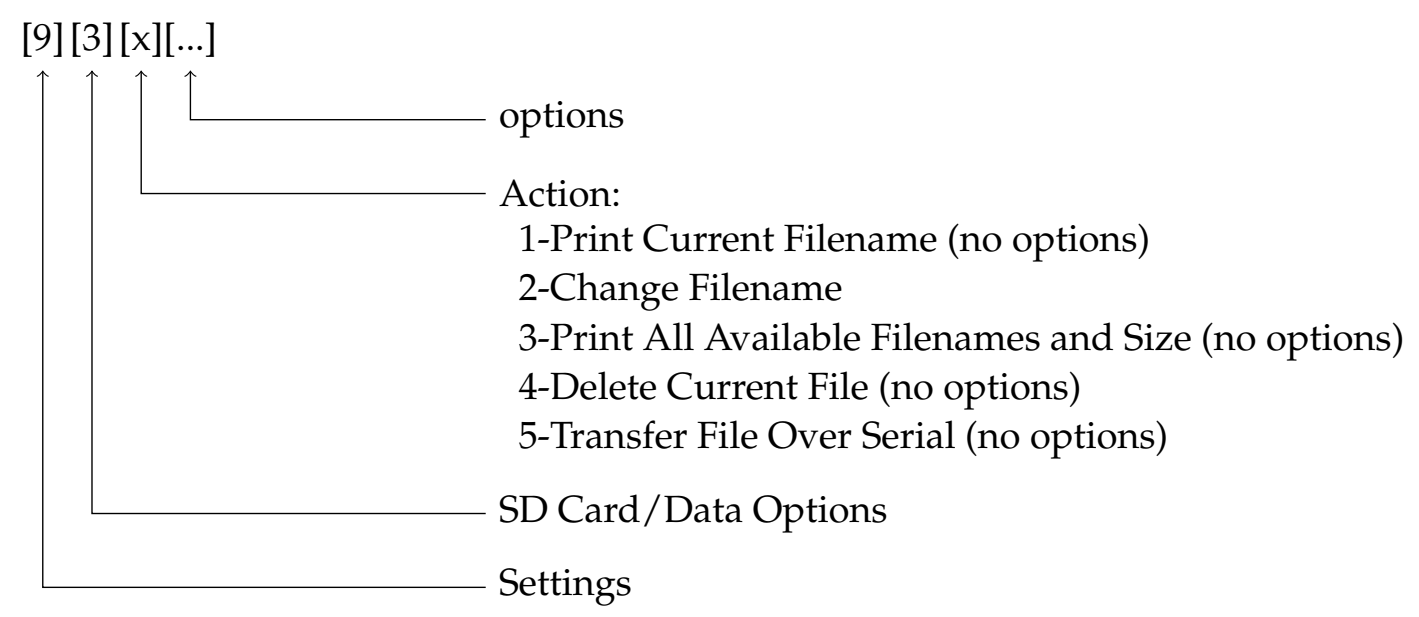

This section describes the micro SD card/data storage options. To utilise these functions a properly formatted micro SD card must be installed in the memory socket.

\section{Print Current Filename}

A command of "931" will print the contents of the filename character array in the motor firmware. This indicates the name of the that data will be written to if a SD card write command is executed within the programme.

\section{Change Filename}

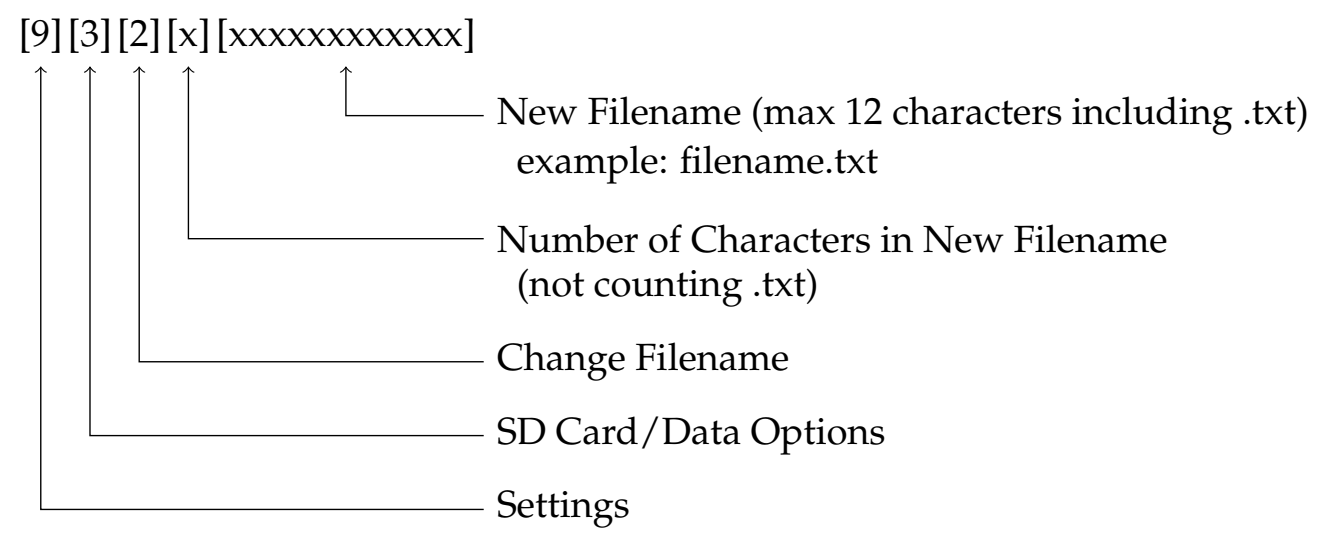

This option is used to change the contents of the filename character array in the motor firmware. Changing the filename would need to take place before writing data to file, deleting the file or transferring the file via serial. Within the firmware, the filename is limited to eight characters with a three character extension (e.g. "filename.txt"). The structure of the "Change Filename" command is illustrated above. 


\section{Print All Available Filenames (with file size)}

A command of "933" will cause the motor to print all available files (and the size in bytes).

\section{Delete Current File}

A command of "934" will delete the file represented by the contents of the filename character array in the motor firmware. To select a file for deletion, use the "Update Filename" command.

\section{Transfer File Over Serial}

A command of "935" will transfer the file represented by the contents of the filename character array in the motor firmware. To select a file for transfer, use the "Update Filename" command.

\section{D.2.4 Debug Mode}

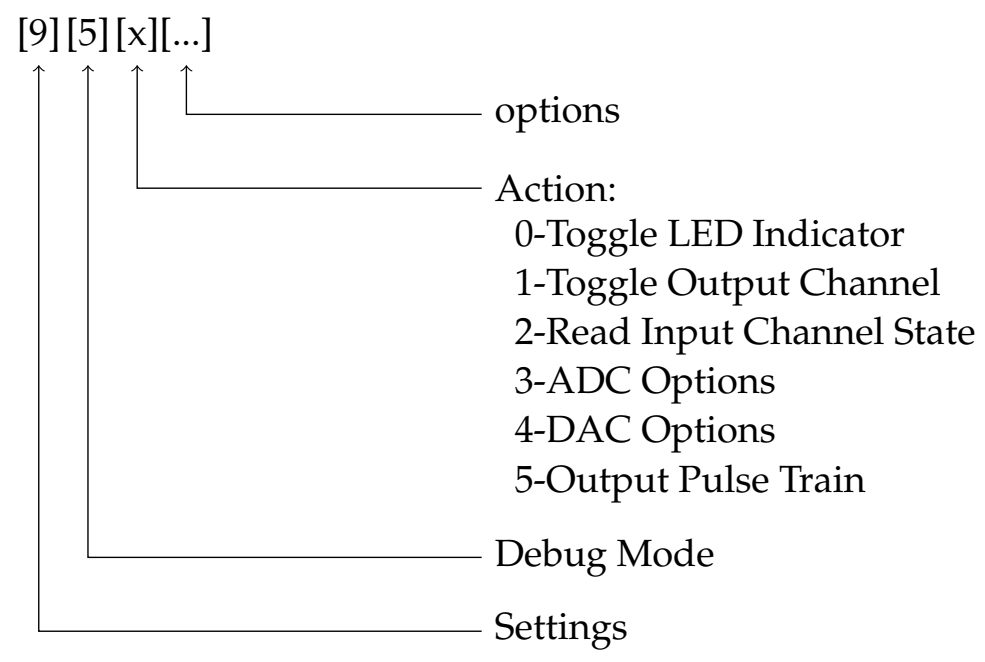

The "Debug Mode" options can be used to test core functions of the motionControl electronics. 


\section{Toggle LED Indicator}

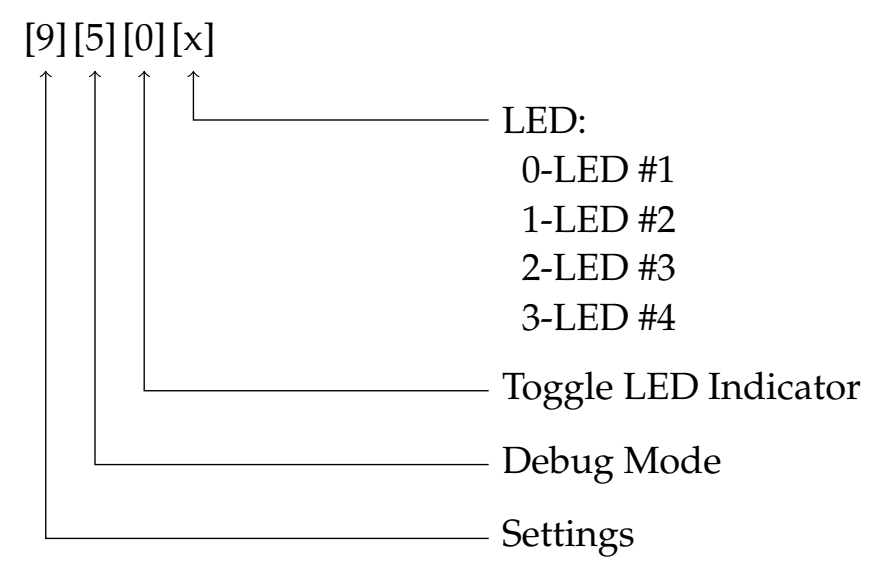

The "Toggle LED Indicator" command can be used to check proper control of the indicator LEDs (numbered 1-4).

\section{Toggle Output Channel}

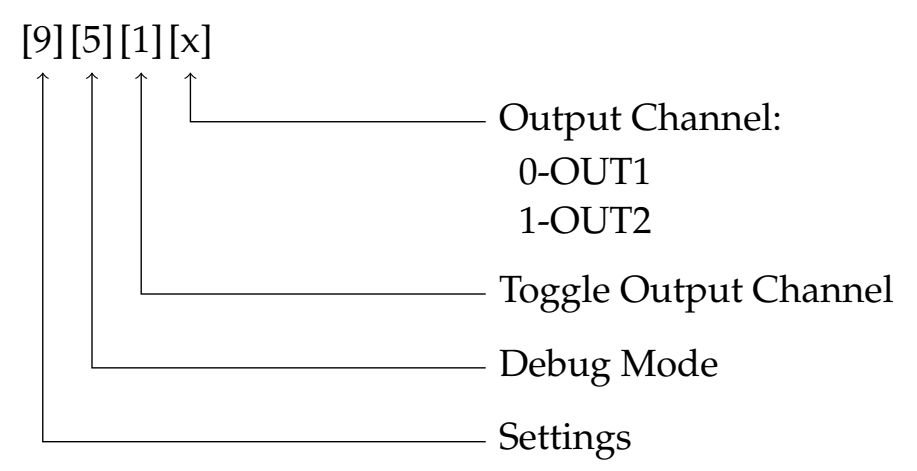

The "Toggle Output Channel" command can be used to check proper control of the digital output lines (OUT1 \& OUT2). If either channel is toggled, the state of the digital line will invert (LOW $\rightarrow$ HIGH or $\mathrm{HIGH} \rightarrow$ LOW).

\section{Read Input Channel State}

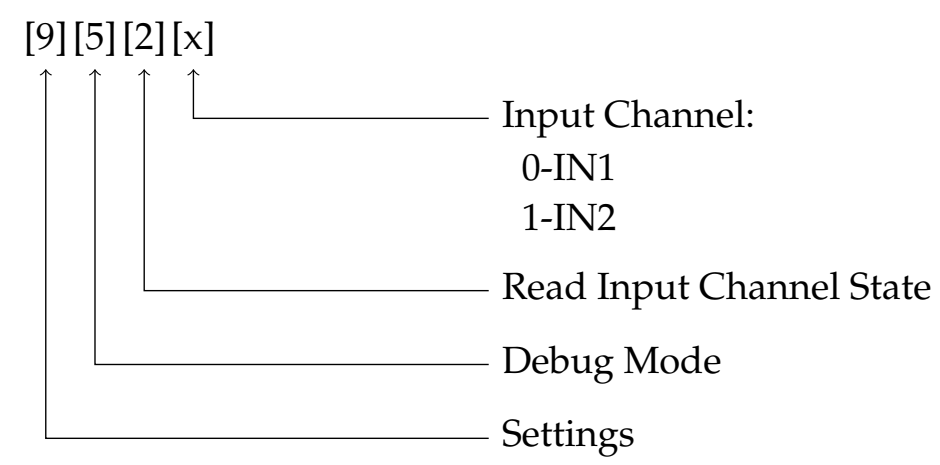


The "Read Input Channel" command can be used to check proper control of the digital input lines (IN1 \& IN2). Each input channel is read independently; a read command will report the current state (HIGH or LOW) of the input channel.

\section{ADC Options}

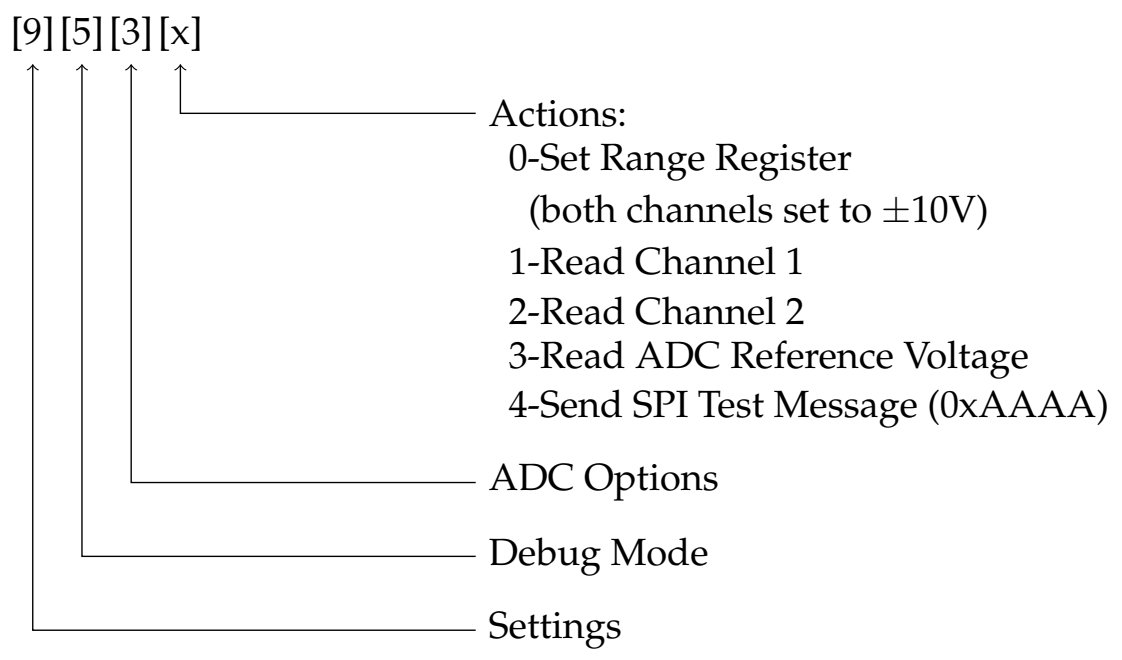

The "ADC Options" refer to the external analogue to digital converter on the rheoTorque shield.

\section{- Set Range Register}

The range register of the external $\mathrm{ADC}$ is used to select one analog input range per analog input channel and to set the ranges for both channels. A command of "9530" sets the channels to $\pm 10 \mathrm{~V}$. By default, the ADC will be set to $\pm 10 \mathrm{~V}$ each time the chip is started (powered on); this command can be used if it's believed the range register has been corrupted (or altered).

\section{- Read Channel 1}

A command of "9531" will return the analogue value of first channel of the external ADC (referred to Channel 0 in the ADC7321 datasheet). In the motionControl system this channel is connected to the torque output of the torque interface card.

\section{- Read Channel 2}

A command of "9532" will return the analogue value of second channel of the external ADC (referred to Channel 1 in the ADC7321 datasheet). In the motionControl system this channel is connected to the reference output of the torque interface card.

\section{- Read ADC Reference Voltage}


A command of "9533" will use the SAM3X8E onboard 12 bit ADC on the Arduino Due board to measure the internal reference voltage of the external ADC. This value should be $\approx 2.5 \mathrm{~V}$.

\section{- Send SPI Test Message}

A command of "9534" can be used to debug the SPI bus of the motionControl electronics. This command will print a 16 bit word (0xAAAA or 0b1010101010101010) to the MOSI line of the SPI bus (Figure D.1).

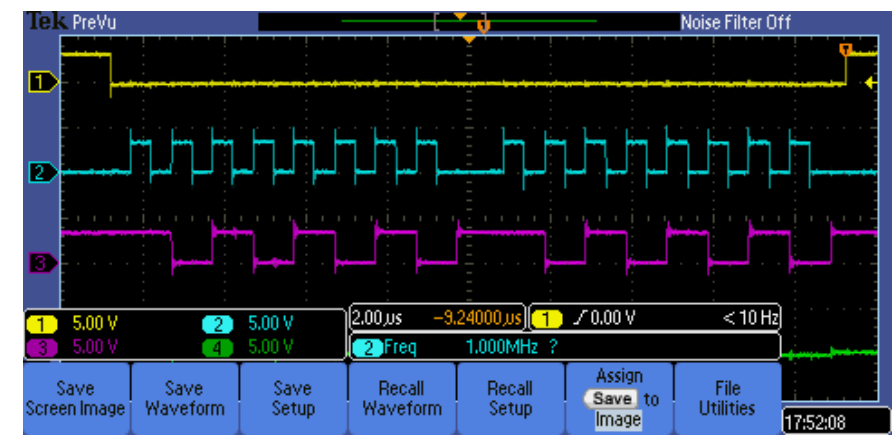

Figure D.1: Oscilloscope screen capture of SPI test message on MOSI line. Visible traces: chip select (yellow), clock (blue), MOSI (pink). Note, MOSI is read on the falling edges of the clock.

\section{DAC Options}

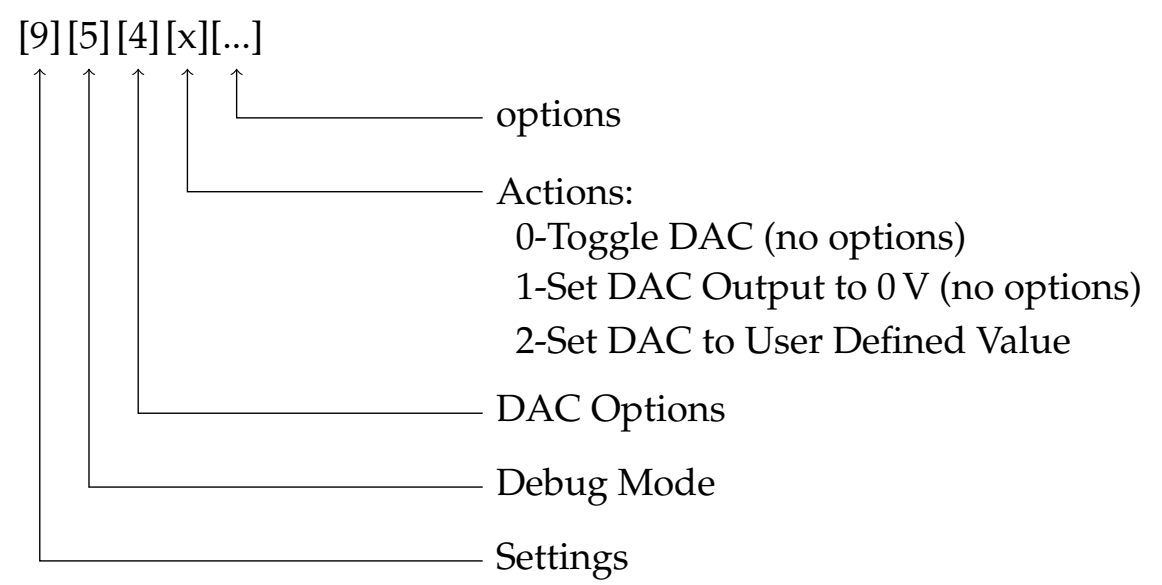

The "DAC Options" refer to the external digital to analogue to converter on the rheoTorque shield.

\section{- Toggle DAC}

A command of "9540" will toggle the power-down mode of the external DAC between enable and disable. 


\section{- Set DAC Output to $0 \mathrm{~V}$}

A command of "9541" will set the analogue output of the external DAC to $0 \mathrm{~V}$.

- Set DAC Output to User Defined Value

$[9][5][4][2][x][x x . x x x]$

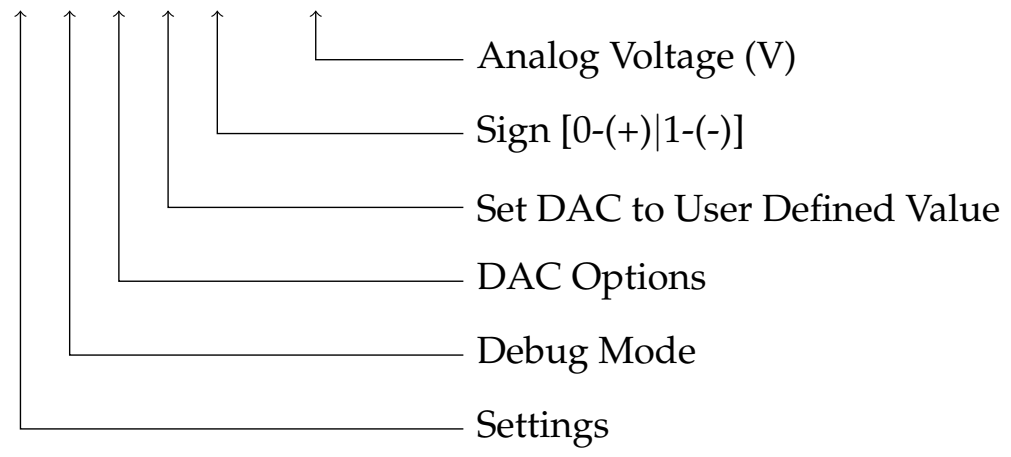

This command is used to set the analogue output of the external DAC to a user defined value between -10 to $+10 \mathrm{~V}$. The structure of the "Set DAC Output" is illustrated above.

\section{D.2.5 Torque Sensor Calibration}

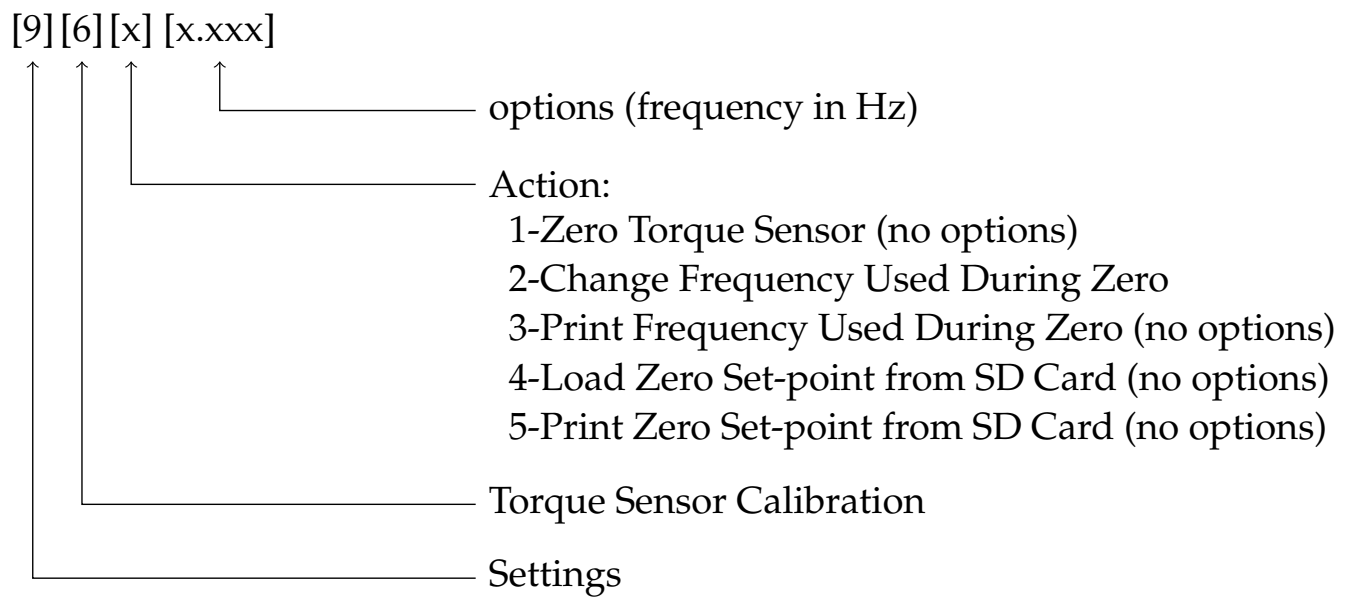

\section{Zero Torque Sensor}

A command of "961" will execute the zero sensor routine. During the zeroing routine the motor will rotate the shaft at the set frequency (this frequency can be changed with the "Update Rotation Frequency" command below). The zero sensor routine takes $\approx 2$ minutes. 


\section{Update Rotation Frequency for Zero Routine}

This command will change the rotation frequency used during the zero sensor routine. To update the frequency use a command of "962[x.xxx]" where the last four digits are the desired frequency (in $\mathrm{Hz}$ ). If updating to a frequency less than $1 \mathrm{~Hz}$, a leading zero must be used (i.e. to update to a frequency of $0.5 \mathrm{~Hz}$ send the command "9620500").

\section{Print Current Rotation Frequency for Zero Routine}

A command of "963" will cause the motor to print the frequency which will be used during subsequent calls of the zeroing routine.

\section{Load Zero Set-Point from SD Card}

A command of "964" will set the analogue output of the external DAC based upon the value stored to the micro SD card on the rheoDueShield.

\section{Print Zero Set-Point from SD Card}

A command of "965" will print (in decimal) the zero value stored from the previous torque sensor routine.

\section{D.2.6 Spectrometer Interface}

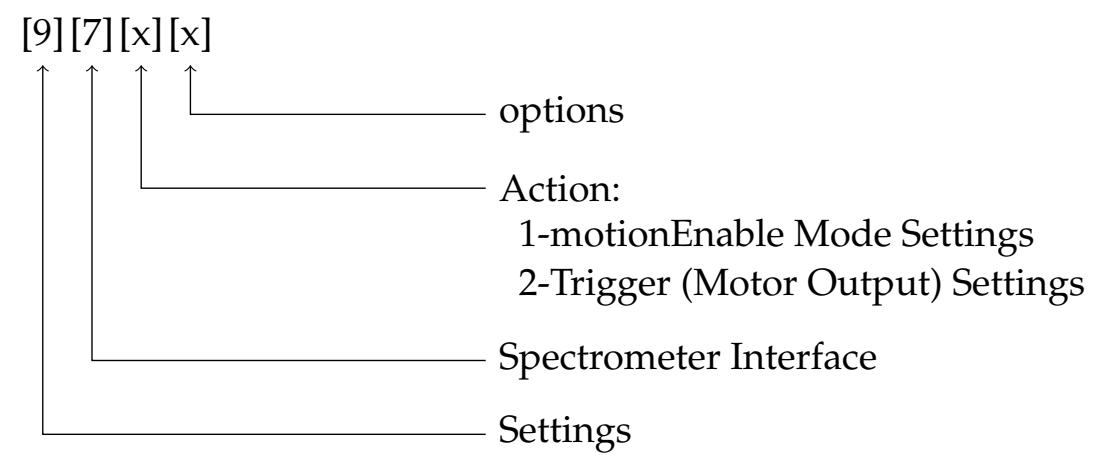


motionEnable Mode Settings

$[9][7][1][x]$

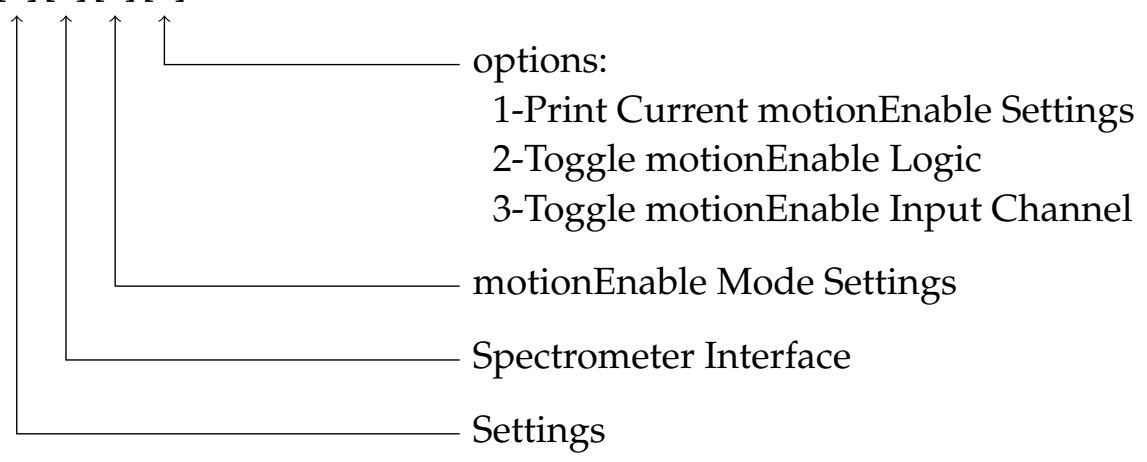

\section{- Print Current motionEnable Settings}

A command of "9711" will print which input channel and the logic setting is currently set for the motionEnable mode.

\section{- Toggle motionEnable Logic}

A command of "9712" will toggle the logic used for the motionEnable mode. Depending on this settings the motor will move when the motionEnable line is set either HIGH or LOW.

\section{- Toggle motionEnable Input Channel}

A command of "9713" will toggle the input channel used for the motionEnable mode between IN1 and IN2.

\section{Trigger (Motor Output) Settings}

$[9][7][2][x]$

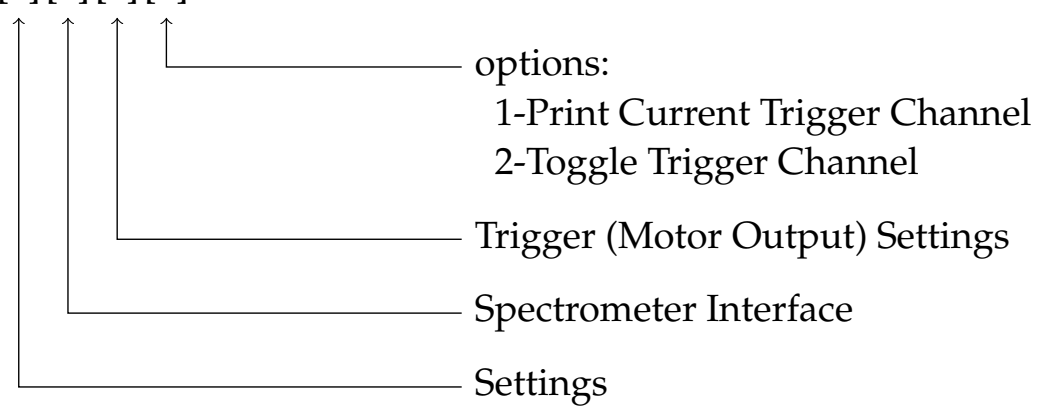

\section{- Print Current Trigger Channel}

A command of "9721" will print which output channel will be used for subsequent Trigger calls (Motor Output). 


\section{- Toggle Trigger Channel}

A command of "9722" will toggle the channel used for subsequent Trigger calls (Motor Output) between OUT1 and OUT2.

\section{D.2.7 Push Button Control}

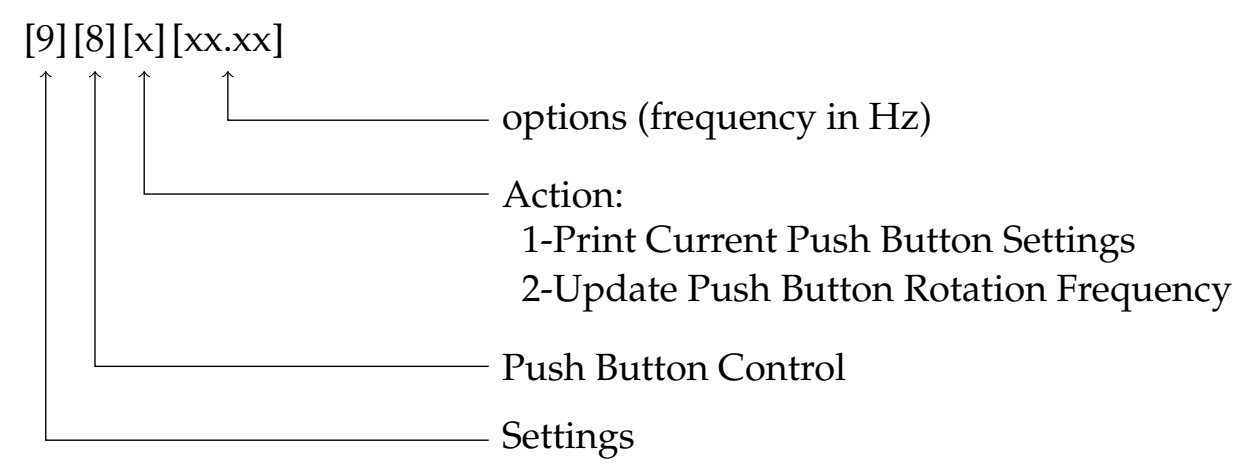

\section{Print Current Push Button Settings}

A command of "981" will cause the motor to print the frequency which will be used during subsequent calls of the move when pushed routine (external trigger button).

\section{Update Push Button Rotation Frequency}

This command will change the rotation frequency used for the move when pushed routine (external trigger button). To update the frequency use a command of "982[xx.xx]" where the last four digits are the desired frequency (in $\mathrm{Hz}$ ). If updating to a frequency less than $10 \mathrm{~Hz}$, a leading zero must be used (i.e. to update to a frequency of $1.5 \mathrm{~Hz}$ send the command “9820150"). 


\section{D.3 Quick Reference}

\begin{tabular}{|l|l|}
\hline Command & Description \\
\hline \hline 0 & Stop Motor and Clear All Parameters \\
\hline \hline $1[\ldots]$ & Single Value Shear Rate Motion Profile \\
\hline \hline $2[\ldots]$ & Oscillatory Shear Motion Profile \\
\hline \hline $3[\ldots]$ & Stepped Shear Rare Motion Profile \\
\hline \hline $4[\ldots]$ & Sawtooth Motion Profile \\
\hline \hline $5[\ldots]$ & Square Wave Motion Profile \\
\hline \hline $6[\ldots]$ & Stepped Motion Profile \\
\hline \hline $7[\ldots]$ & Single Value Shear Stress Motion Profile \\
\hline \hline 911 & Echo \\
\hline 912 & Print Firmware Version \\
\hline 913 & Print Parameters \\
\hline \hline 920 & Print Motor Settings \\
\hline 921 & Toggle Motor Enable \\
\hline 9221 & Print Current StepsPerRev Value \\
\hline $9222[x x x x x]$ & Update StepsPerRev Value \\
\hline 923 & Toggle Dampening Mode \\
\hline 9241 & Print Current Drive Current Value \\
\hline $9242[x x x x]$ & Update Drive Current Value \\
\hline 9251 & Delete User Defined Parameters File \\
\hline 9252 & Create User Defined Parameters File \\
\hline 9253 & Print User Defined Parameters File \\
\hline 9254 & Print Default Parameters File \\
\hline 9255 & Load Values from User Defined Parameters File \\
\hline 9256 & Load Values from Default Parameters File \\
\hline \hline 931 & Print Current Filename \\
\hline $932[x][x x x x x x x x x x x]$ & Update Filename \\
\hline 933 & Print All Available Files \\
\hline 934 & Delete Current File \\
\hline 935 & Transfer Current File via Serial \\
\hline \hline 94 & Unused) \\
\hline \hline $950[x]$ & \\
\hline $951[x]$ & Toggle Output Channel \\
\hline & \\
\hline
\end{tabular}




\begin{tabular}{|l|l|}
\hline $952[x]$ & Read State of Input Channel \\
\hline 9530 & Set External ADC Range Register \\
\hline 9531 & Read External ADC Channel 1 \\
\hline 9532 & Read External ADC Channel 2 \\
\hline 9533 & Read External ADC Reference Voltage \\
\hline 9534 & Send SPI Test Message \\
\hline 9540 & Toggle External DAC \\
\hline 9541 & Set External DAC Output to 0V \\
\hline $9542[x][x x . x x x]$ & Set External DAC Output to User Defined Value \\
\hline \hline 961 & Zero Torque Sensor \\
\hline $962[x . x x x]$ & Update Rotation Frequency For Zero Routine \\
\hline 963 & Print Current Rotation Frequency For Zero Routine \\
\hline 964 & Load Zero Set-Point from SD Card \\
\hline 965 & Print Zero Set-Point from SD Card \\
\hline \hline 9711 & Print Current motionEnable Settings \\
\hline 9712 & Toggle motionEnable Logic \\
\hline 9713 & Toggle motionEnable Input Channel \\
\hline 9721 & Print Current Trigger Channel \\
\hline 9722 & Toggle Trigger Channel \\
\hline \hline 981 & Print Current Push Button Settings \\
\hline $982[x x . x x]$ & Update Push Button Rotation Frequency \\
\hline
\end{tabular}

